\title{
Aspectos combinatórios \\ da geometria de espaços \\ de Banach $C(K)$ com a \\ propriedade de Grothendieck
}

\author{
Christina Brech \\ DISSERTAÇÃO APRESENTADA \\ AO \\ Instituto de Matemática e Estatística \\ $\mathrm{DA}$ \\ Universidade de SÃo Paulo \\ PARA \\ OBTENÇÃO DO GRAU \\ $\mathrm{DE}$ \\ Mestre em Matemáticia \\ Área de Concentração: Matemática \\ Orientador: Prof. Dr. Piotr Koszmider
}

Durante a elaboração deste trabalho, o autor recebeu apoio financeiro da CAPES e da FAPESP (processo 02/04531-6). 


\title{
Aspectos combinatórios da geometria de espaços de Banach $C(K)$ com a propriedade de Grothendieck
}

\author{
Este exemplar corresponde à redação \\ final da dissertação de mestrado devidamente \\ corrigida e defendida por Christina Brech \\ e aprovada pela comissão julgadora.
}

São Paulo, março de 2004.

Banca examinadora:

- Prof. Dr. Piotr Koszmider - IME-USP

- Profa. Dra. Ofélia Teresa Allas - IME-USP

- Prof. Dr. Jorge Tulio Mujica - IMECC-UNICAMP 
Ao meu avô Theodoro Bayma de Carvalho, por razôes que não sei expressar. 


\section{Agradecimentos}

Agradeço a todos aqueles que me apoiaram de alguma forma durante a elaboração deste
trabalho.

Agradeço especialmente ao meu orientador, Prof. Piotr Koszmider, pelo incentivo e paciência constantes.

Agradeço aos meus pais e irmãos, por todo o apoio que me deram.

Agradeço a todos os meus professores, especialmente às Profas. Mary Lilian Lourenço e Zara Issa Abud, pela orientação durante a graduação e pela amizade.

Agradeço a todos os meus amigos, especialmente ao Demo (Leandro), pelo carinho e paciência durante estes dois anos, e à Natalia, pela amizade e companhia nas boas e más horas do mestrado.

Agradeço, por fim, à CAPES e à FAPESP pelo apoio financeiro. 
"Deus existe uma vez que a matemática é consistente, e o Diabo existe dado que não podemos demonstrá-lo."

André Weil 


\section{Resumo}

O principal objetivo deste trabalho é estudar certos aspectos da interação entre duas teorias: a teoria clássica de espaços de Banach de funções contínuas num espaço compacto Hausdorff com a norma do supremo e os métodos modernos de combinatória infinitária e forcing, aplicados na construção e análise de tais espaços. Usando forcing e combinatória infinitária podemos definir e construir álgebras de Boole para as quais obtemos, usando a dualidade de Stone, um espaço compacto Hausdorff $K$. Consideramos então o espaço de Banach $C(K)$ e analisamos de que forma as propriedades analíticas deste espaço são influenciadas por propriedades combinatórias da álgebra de Boole. Neste contexto, enfatizamos a propriedade de Grothendieck e a estrutura de subespaços complementados. Os espaços desta forma aqui estudados são: o $l_{\infty}, \mathrm{o} l_{\infty} / c_{0}$, um espaço construído por Haydon e um novo espaço aqui apresentado. Todos eles têm a propriedade de Grothendieck.

\section{Abstract}

The main purpose of this work is to study some aspects of the interaction between two theories: the classical theory of Banach spaces of continuous functions on a compact Hausdorff space with the supremum norm and the modern methods of infinitary combinatorics and forcing, applied in the constructions and analysis of such spaces. Using forcing and infinitary combinatorics we can define and construct Boolean algebras for which we obtain, using Stone duality, a compact Hausdorff space $K$. We consider then the Banach space $C(K)$ and we analyze in which way the analytic properties of this space are influenced by the combinatorial properties of the Boolean algebra. In this context, we emphasize the Grothendieck property and the structure of complemented subspaces. The spaces of this form studied here are: $l_{\infty}, l_{\infty} / c_{0}$, a space constructed by Haydon and a new example introduced here. All of them have the Grothendieck property. 


\title{
Notação
}

\author{
$\mathbb{N} \quad$ o conjunto dos números naturais \\ $\mathbb{R} \quad$ o conjunto dos números reais \\ $\omega \quad$ o cardinal enumerável infinito \\ $\omega_{1} \quad$ o menor cardinal não enumerável \\ $\omega_{2} \quad$ o segundo cardinal não enumerável \\ $2^{\omega} \quad$ o cardinal do contínuo \\ $\wp(X) \quad$ o conjunto de todos os subconjuntos de $X$ \\ $|X|$ a cardinalidade de $X$ \\ $\operatorname{Ker}(f)$ o núcleo da função $f$ \\ $\operatorname{Im}(f)$ a imagem da função $f$ \\ $\left.f\right|_{X} \quad$ a função $f$ restrita ao conjunto $X$ \\ $\|x\| \quad$ a norma de $x$ \\ $\chi_{a}$ a função característica de $a$ \\ $l_{\infty} \quad$ o espaço de Banach das seqüências limitadas \\ $c_{0} \quad$ o espaço de Banach das seqüências convergentes a 0
}




\title{
Sumário
}

\author{
Introdução
}

1 Álgebras de Boole e espaços compactos

1.1 Álgebras de Boole . . . . . . . . . . . . . . . . . . . 7

1.1 .1 Definição e exemplos . . . . . . . . . . . . . . . 7

1.1 .2 Álgebras geradas . . . . . . . . . . . . . . 8

1.1.3 Filtros e ultrafiltros . . . . . . . . . . . . . . . 11

1.1.4 Homomorfismos . . . . . . . . . . . . . . . . . . 13

1.1.5 Álgebras completas . . . . . . . . . . . . . . . . . . . . 17

1.2 Espaços topológicos . . . . . . . . . . . . . . . . . . . . 18

1.3 A dualidade de Stone . . . . . . . . . . . . . . . . . . . . . . 23

1.3.1 O espaço de Stone de uma álgebra . . . . . . . . . . . . . 23

1.3.2 A representação de Stone . . . . . . . . . . . . . . . . 25

1.3 .3 A dualidade de Stone . . . . . . . . . . . . . . . . 28

1.4 Algumas traduções . . . . . . . . . . . . . . . . . . . . 31

1.4.1 Cardinalidade de álgebras e propriedades dos espaços de Stone . . . 31

1.4.2 FinCofin $(\mathbb{N})$ como imagem homomorfa e seqüências convergentes não triviais . . . . . . . . . . . . . . . . . 32

1.4.3 $\wp(\mathbb{N})$ como imagem homomorfa e $\beta \mathbb{N}$ como subespaço . . . . . . . . 32 
1.4.4 Álgebras completas e espaços extremamente desconexos . . . . . . . 34

1.4.5 Álgebras superatômicas e espaços dispersos . . . . . . . . . . . . . . 34

1.4.6 Retratos de álgebras e retratos de espaços topológicos . . . . . . . . 35

2 Espaços de Banach $\quad 37$

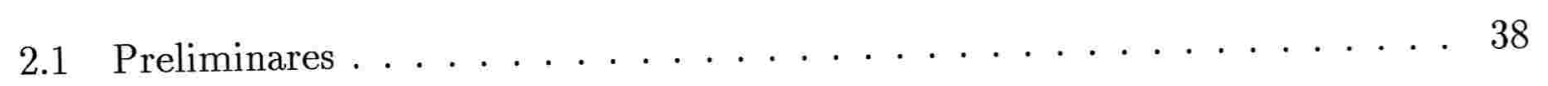

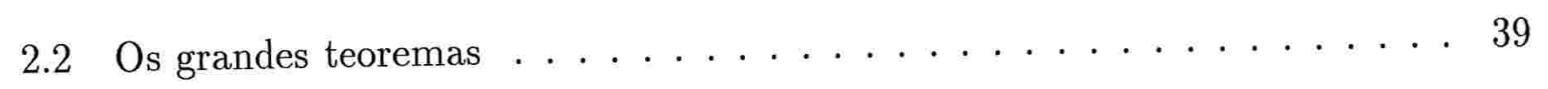

2.3 A topologia fraca . . . . . . . . . . . . . . . 40

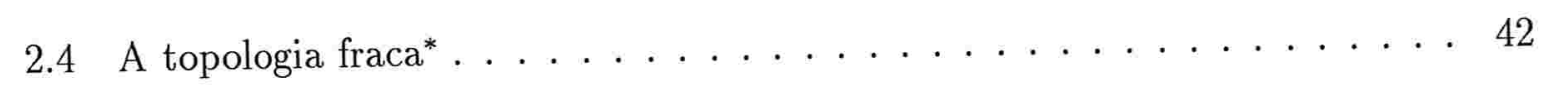

2.5 o operador adjunto . . . . . . . . . . . . 43

2.6 Operações em espaços de Banach . . . . . . . . . . . . . . . 45

2.6.1 Subespaços complementados . . . . . . . . . . . . 45

2.6.2 O método de decomposição de Pełczyński . . . . . . . . . . . . . . . 48

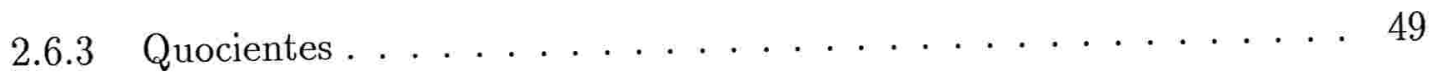

2.7 A propriedade de Grothendieck . . . . . . . . . . . . . . . 50

3 Espaços de Banach da forma $C(K)$

3.1 Resultados úteis . . . . . . . . . . . . . . . 56

3.1.1 O Teorema de Stone-Weierstrass . . . . . . . . . . . . . 57

3.1 .2 O Teorema de Representação de Riesz . . . . . . . . . . . . . 58

$3.2 \quad$ A topologia fraca em $C(K) \ldots \ldots \ldots \ldots \ldots \ldots$

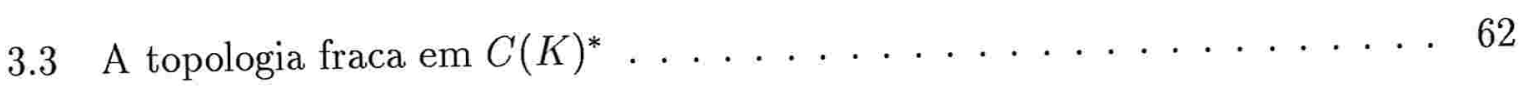

3.4 A topologia fraca ${ }^{*}$ em $C(K)^{*} \ldots \ldots \ldots \ldots \ldots \ldots \ldots$

3.5 Subespaços e subespaços complementados . . . . . . . . . . . 65

3.5 .1 Alguns exemplos . . . . . . . . . . . . . 65

3.5 .2 o $c_{0}$ como subespaço . . . . . . . . . . . 67 
$3.5 .3 \quad \mathrm{o} l_{\infty}$ como subespaço . . . . . . . . . . . . 72

3.6 Lemas combinatórios . . . . . . . . . . . . . . . . . . 73

3.7 Operadores fracamente compactos em $C(K) \ldots \ldots \ldots$. . . . . . 78

3.8 A propriedade de Grothendieck em $C(K) \ldots \ldots \ldots$. . . . . . . 83

3.8 .1 Uma caracterização . . . . . . . . . . . . . . . . . . . . . . . . . 84

3.8.2 A propriedade de completude subseqüencial . . . . . . . . . . 86

4 O método de forcing $\quad 89$

4.1 Consistência e modelos para ZFC . . . . . . . . . . . . . . . . . 91

4.2 A construção de $M[G] \ldots \ldots \ldots$. . . . . . . . . . . . . 96

4.3 As relações $\Vdash$ e $\mathbb{}^{*} \ldots \ldots \ldots 3$

4.4 A equivalência das relações $\Vdash \mathbb{e}^{*} \ldots \ldots$. . . . . . . . . . . . . 109

4.5 Preservação de cardinais . . . . . . . . . . . . . . . . . 115

4.6 O forcing de Cohen . . . . . . . . . . . . . . . . . 116

4.7 O forcing de Sacks . . . . . . . . . . . . . . . . . . . . 119

4.7.1 O forcing de Sacks simples . . . . . . . . . . . . . . . 120

4.7 .2 O produto de forcing's de Sacks . . . . . . . . . . . . . . . . 122

$5 \quad \mathrm{O}$ espaço $l_{\infty} \equiv C(\beta \mathbb{N})$ e a álgebra $\wp(\mathbb{N}) \quad 127$

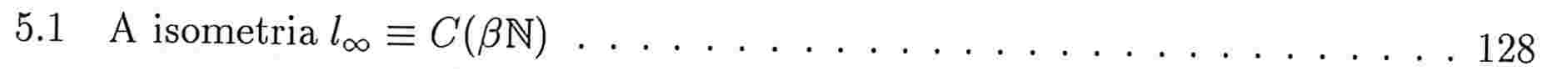

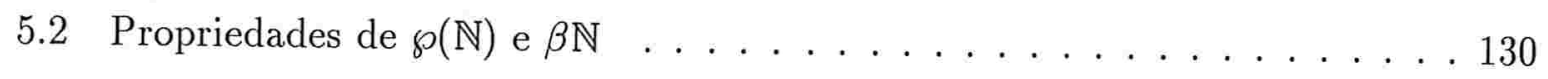

5.3 Subespaços complementados do $l_{\infty} \ldots \ldots \ldots \ldots$. . . . . . . . . . . . . . . . .

6 O espaço $l_{\infty} / c_{0} \equiv C\left(\omega^{*}\right)$ e a álgebra $\wp(N) / F$ in $\quad 139$

6.1 A isometria $l_{\infty} / c_{0} \equiv C\left(\omega^{*}\right) \ldots \ldots \ldots \ldots \ldots \ldots$

6.2 Propriedades de $\wp(\mathbb{N}) / F$ in e de $\omega^{*} \ldots \ldots \ldots \ldots$. . . . . . . . . . 142

6.3 Subespaços complementados do $l_{\infty} / c_{0} \ldots \ldots \ldots \ldots$. . . . . . . . . . . . . . 
7 Pequenos espaços de Grothendieck $C(K)$

7.1 A álgebra de Haydon . . . . . . . . . . . . . . . 161

7.2 A densidade de espaços de Grothendieck $C(K) \ldots \ldots \ldots \ldots 6$

Referências Bibliográficas 


\section{Introdução}

Este trabalho tem por objetivo o estudo de certos aspectos da interação entre a teoria clássica de espaços de Banach de funções contínuas num espaço compacto Hausdorff e os métodos modernos de combinatória infinitária e forcing ${ }^{1}$, aplicados na construção e análise destes espaços topológicos. Mais especificamente, estudamos de que forma certos métodos combinatórios se relacionam com algumas propriedades de espaços de Banach de funções contínuas: seus subespaços complementados ${ }^{2}$ e a propriedade de Grothendieck ${ }^{3}$.

Os espaços $l_{\infty}$ e $l_{\infty} / c_{0}$, clássicos espaços de Banach da forma $C(K)$ que têm a propriedade de Grothendieck e subespaços complementados bastante específicos, são aqui estudados. A fim de obter mais exemplos de espaços de Grothendieck, construímos álgebras de Boole, através do forcing e de indução transfinita e, usando a dualidade de Stone ${ }^{4}$, obtemos espaços compactos Hausdorff $K$, para os quais consideramos o espaço de Banach $C(K)$. O espaço para o qual usamos indução transfinita é um espaço construído por Haydon em 1981 (veja [Ha]), que não tem cópias complementadas de $c_{0}$ nem de $l_{\infty}$. O exemplo construído usando o forcing é um novo espaço de Grothendieck com propriedades interessantes $^{5}$ (veja [Bre]). Além da propriedade de Grothendieck, destacamos em alguns

\footnotetext{
${ }^{1} \mathrm{O}$ forcing é um método combinatório, cuja aplicação é a obtenção de "modelos" para a matemática e, portanto, de resultados de consistência.

${ }^{2}$ Dizemos que um subespaço $Y$ de um espaço de Banach $X$ é complementado se existe $P: X \rightarrow Y$ linear, contínua, sobrejetora e tal que $\left.P\right|_{Y}=I d$.

${ }^{3}$ Dizemos que um espaço de Banach $X$ tem a propriedade de Grothendieck se a convergência fraca de seqüências coincide com a convergência fraca* no espaço dual $X^{*}$.

${ }^{4}$ A dualidade de Stone tem caráter funtorial, entre a categoria das álgebras de Boole e a dos espaços compactos, Hausdorff e zero-dimensionais.

${ }^{5} \mathrm{O}$ espaço que construímos possui a propriedade de Grothendieck e tem densidade menor que o cardinal do contínuo. Convém notar que, como usamos o forcing para obtê-lo, tal espaço existe apenas consistentemente.
} 
exemplos a estrutura de subespaços complementados do espaço de Banach em questão.

O trabalho é composto por quatro capítulos introdutórios seguidos por três capítulos mais avançados. Nos quatro primeiros capítulos são abordadas as teorias posteriormente utilizadas. Mais especificamente, no Capítulo 1 são apresentados tópicos da teoria de álgebras de Boole e da teoria de espaços compactos Hausdorff e a dualidade de Stone. No Capítulo 2 apresentamos os principais resultados da teoria de espaços de Banach em geral, as topologias fraca e fraca-estrela, bem como diversos resultados sobre subespaços complementados e introduzimos a propriedade de Grothendieck. No Capítulo 3, apresentamos resultados sobre espaços de Banach de funções contínuas, incluindo propriedades das topologias fraca e fraca-estrela, subespaços complementados e operadores fracamente compactos. Analisamos, neste contexto, como os espaços com a propriedade de Grothendieck se comportam. E no Capítulo 4 apresentamos os elementos fundamentais do forcing, isto é, como trabalhamos com ele e de que forma pode nos ser útil, e analisamos propriedades de alguns forcing's específicos.

No quinto capítulo estudamos o espaço ${ }^{6} l_{\infty} \equiv C(\beta \mathbb{N})$, como primeiro exemplo de um espaço de Grothendieck da forma $C(K)$. Apresentamos a álgebra de Boole $\wp(\mathbb{N})$, cujo espaço de Stone $^{7}$ é o $\beta \mathbb{N}$, e algumas traduções de suas propriedades a propriedades dos espaços $\beta \mathbb{N}$ e $l_{\infty}$. Finalmente, analisamos os subespaços complementados do $l_{\infty}$, obtendo que ele é primo ${ }^{8}$. Por ser o mais clássico espaço de Banach da forma $C(K)$ não separável, o $l_{\infty}$ serve como contexto para os próximos capítulos, isto é, ao analisarmos outros espaços de Banach, consideramos as propriedades que o $l_{\infty}$ possui para formular perguntas e fazer conjecturas.

No Capítulo 6, apresentamos o espaço ${ }^{9} l_{\infty} / c_{0} \equiv C\left(\omega^{*}\right)$, fazendo um estudo paralelo ao feito no capítulo anterior, novamente provando que tal espaço tem a propriedade de Grothendieck. Neste caso, porém, estudamos a álgebra $\wp(\mathbb{N}) / F i n$, cujo espaço de Stone é o $\omega^{*}$ e traduções de propriedades entre estes objetos. Ao analisarmos os subespaços complementados de $l_{\infty} / c_{0}$ aparecem, diferentemente do que ocorre com o $l_{\infty}$, resultados de

\footnotetext{
${ }^{6} \beta \mathbb{N}$ é o compactificado de Čech-Stone dos naturais.

${ }^{7} \mathrm{O}$ espaço de Stone de uma álgebra de Boole é o espaço compacto Hausdorff e zero-dimensional obtido pela dualidade de Stone.

${ }^{8}$ Dizemos que um espaço de Banach $X$ de dimensão infinita é primo se para todo $Y$ subespaço complementado em $X$ de dimensão infinita, tem-se que $Y$ é isomorfo a $X$.

${ }^{9} \omega^{*}=\beta \mathbb{N} \backslash \mathbb{N}$.
} 


\section{INTRODUÇÃO}

consistência, conseqüências de questões independentes relacionadas à álgebra $\wp(\mathbb{N}) / F$ in . Assim, a estrutura do espaço $l_{\infty} / c_{0}$ é bastante influenciada por hipóteses conjuntísticas adicionais.

No último capítulo fazemos duas construções de álgebras de Boole e de espaços de Banach da forma $C(K)$ e analisamos suas propriedades. Cada uma delas usa um método diferente: a indução transfinita e o forcing, sendo que construções feitas com o forcing existem apenas consistentemente. Por indução transfinita, fazemos a mesma construção feita em [Ha], obtendo uma álgebra de Boole, tal que se $K$ é seu espaço de Stone, então $C(K)$ não tem cópias complementadas de $c_{0}$ nem de $l_{\infty}$. Além disso, tal espaço possui a propriedade de Grothendieck. Usando o forcing, construímos uma álgebra de Boole (a mesma de [JK]) e mostramos que se $K$ é seu espaço de Stone, então $C(K)$ é um espaço com a propriedade de Grothendieck, que possui densidade ${ }^{10} \omega_{1}<2^{\omega}$. Combinado a um resultado já conhecido, este novo espaço fornece a independência da existência de um espaço $C(K)$ de Grothendieck com densidade menor que o cardinal do contínuo $2^{\omega}$ (veja $[\mathrm{Bre}])$.

\footnotetext{
${ }^{10} \mathrm{~A}$ densidade de um espaço de Banach $X$ é o menor cardinal para o qual existe $D \subseteq X$ denso com tal cardinalidade. $\omega_{1}$ é o menor cardinal não enumerável e $2^{\omega}$ é a cardinalidade de $\wp(\mathbb{N})$.
} 


\section{Capítulo 1}

\section{Álgebras de Boole e espaços compactos}

Nosso objetivo neste capítulo se divide no estudo de três tópicos: as álgebras de Boole e suas propriedades, espaços compactos Hausdorff, e a dualidade de Stone.

No que se refere ao estudo das álgebras de Boole, estudamos objetos como subálgebras, ideais, filtros e ultrafiltros e álgebras geradas. Fazemos então um estudo dos homomorfismos entre álgebras de Boole: funções entre álgebras de Boole que preservam sua estrutura. Por fim, estudamos as álgebras completas ${ }^{1}$, que são bastante particulares, mas muito interessantes. O resultado mais importante desta seção é o seguinte:

Teorema (da Extensão de Sikorski). Sejam $A, B, C$ álgebras de Boole e $h: A \rightarrow C$ um homomorfismo. Se $A$ é subálgebra de $B$ e $C$ é completa, então existe um homomorfismo $\tilde{h}: B \rightarrow C$ tal que $\left.\tilde{h}\right|_{A}=h$.

A seção sobre os espaços compactos Hausdorff é composta por conceitos como peso, densidade, normalidade, separabilidade e metrizabilidade, bem como por condições equivalentes à compacidade. O principal resultado aqui é:

Teorema (de Extensão de Tietze). Seja $X$ um espaço topológico normal. Se $M \subseteq X$ é um subconjunto fechado de $X$ e $f: M \rightarrow \mathbb{R}$, uma função contínua, então existe uma

\footnotetext{
${ }^{1}$ Dizemos que uma álgebra de Boole $A$ é completa se para toda anticadeia $\left(a_{n}\right)_{n \in \mathbb{N}} \subseteq A$, tem-se que $\sum_{n \in \mathbb{N}} a_{n} \in A$.
} 
função contínua $\tilde{f}: X \rightarrow \mathbb{R}$ tal que $\left.\tilde{f}\right|_{M}=f e\|\tilde{f}\|=\|f\|$.

Nosso interesse em estudar espaços compactos Hausdorff tem sua motivação no objetivo de estudar (a partir do Capítulo 3) espaços de Banach da forma $C(K)$, onde $K$ é um tal espaço topológico. Já as álgebras de Boole nos interessam por sua forte relação com certos espaços compactos Hausdorff:

Teorema (de Representação de Stone). Para cada álgebra de Boole A, existe um espaço topológico compacto Hausdorff e zero-dimensional $K$ tal que $A$ é isomorfa a uma base de abertos-fechados para a topologia de $K$.

Reciprocamente, se $K$ é um espaço topológico compacto Hausdorff zero-dimensional, então os abertos-fechados formam uma álgebra de Boole.

Os principais exemplos de espaços de Stone estudados ${ }^{3}$ são o compactificado de ČechStone dos naturais $(\beta \mathbb{N})$ e o $\omega^{*}=\beta \mathbb{N} \backslash \mathbb{N}$. O resultado acima permite-nos fazer traduções de uma teoria para a outra. Mostramos, por exemplo, que um espaço de Stone possui uma cópia do $\beta \mathbb{N}$ se e somente se sua álgebra de Boole tem $\wp(\mathbb{N})$ como imagem homomorfa.

Na realidade, a dualidade de Stone, entre álgebras de Boole e espaços de Stone, vai além do resultado enunciado, como veremos na Seção 1.3.3. Temos uma dualidade que leva subálgebras de álgebras de Boole em funções contínuas de espaços de Stone, bem como homomorfismos entre álgebras em subespaços fechados de espaços de Stone. Assim, além de fazer traduções entre propriedades destes objetos, podemos fazer traduções entre suas sub-estruturas (subálgebras e subespaços) e funções que preservam sua estrutura (homomorfismos e funções contínuas). Trabalhar em uma das linguagens pode ser, muitas vezes, mais natural; e tal dualidade permite-nos fazê-lo.

Por fim, na última seção abordamos diversas traduções entre as duas teorias. Algumas delas serão utilizadas nos capítulos seguintes. A principal, diz respeito aos retratos de espaços topológicos e retratos de álgebras de Boole.

Como referência para os temas deste capítulo, indicamos [Kopp] para as Seções 1.1,

${ }^{2}$ Dizemos que um espaço topológico é zero-dimensional se ele possui uma base de abertos-fechados.

${ }^{3} \mathrm{O}$ produto de Tychonoff $2^{\kappa}$ do espaço $\{0,1\}, \kappa$ vezes; o compactificado de Alexandroff $\Gamma(\kappa)$ de um espaço discreto com $\kappa$ elementos; e o espaço $[0, \alpha]$ com a topologia da ordem, onde $\alpha$ é um ordinal, também são clássicos espaços de Stone. Porém, todos eles possuem seqüências convergentes não triviais, o quê não nos interessa, uma vez que, neste caso, o espaço $C(K)$ não tem a propriedade de Grothendieck. 
1.3 e 1.4 e [Eng] para a Seção 1.2.

\section{1 Álgebras de Boole}

Nesta seção, fazemos uma apresentação da teoria das álgebras de Boole. Como já dissemos, tais objetos nos interessam, uma vez que pela dualidade de Stone (a ser posteriormente abordada) eles nos fornecem espaços compactos, a partir dos quais temos espaços de Banach de funções contínuas, como queremos.

\subsubsection{Definição e exemplos}

Comecemos com algumas definições:

Definição 1.1. Dizemos que $(A,+, \cdot,-, 0,1)$ é uma álgebra de Boole se $A$ é um conjunto, $0 \neq 1 \in A,+e \cdot$ são operaçôes binárias em $A$, - é uma operação unária em $A$ tais que para todos $a, b, c \in A$ temos
(1) $a+b=b+a$
(1') $a \cdot b=b \cdot a$
(2) $(a+b)+c=a+(b+c)$
$\left(2^{\prime}\right)(a \cdot b) \cdot c=a \cdot(b \cdot c)$
(3) $a \cdot(b+c)=a \cdot b+a \cdot c$
$\left(3^{\prime}\right) \quad a+(b \cdot c)=(a+b) \cdot(a+c)$
(4) $a+a \cdot b=a$
(4') $a \cdot(a+b)=a$
(5) $a+(-a)=1$
$\left(5^{\prime}\right) \quad a \cdot(-a)=0$

Queremos agora ter uma idéia dos métodos através dos quais podemos obter álgebras de Boole, para posteriormente usá-los. Um método para criar álgebras de Boole é considerar subconjuntos de álgebras de Boole com boas propriedades (fechados por uniões e intersecções finitas e complementos):

Definição 1.2. Dizemos que $B \subseteq A$ é uma subálgebra de $A$ se $B$, munido das operações de A, é uma álgebra de Boole.

Podemos definir uma ordem parcial em toda álgebra de Boole:

Definição 1.3. Dada uma álgebra de Boole $A$, e dados $a, b \in A$, definimos $a \leq b$ se $a=a \cdot b$. 
Tal ordem nos fornece boas intuições para trabalhar com álgebras de Boole. Vejamos, por exemplo, o que ocorre com corpos de conjuntos:

Exemplo 1.4 (corpo de conjuntos). Seja $X$ um conjunto e $A \subseteq \wp(X)$ um corpo de conjuntos $^{4}$. É fácil ver que $(A, \cup, \cap, X \backslash \cdot \emptyset, X)$ é uma álgebra de Boole. Neste caso, se $a, b \in A$, então $a \leq b$ se, e somente se, $a \subseteq b$. Além disso, A é uma subálgebra de $\wp(X)$.

Outro método interessante de criar álgebras de Boole é considerar cadeias de álgebras de Boole:

Exemplo 1.5 (cadeia de álgebras de Boole). Se $\gamma$ é um ordinal e para todo $\alpha<\gamma$, $A_{\alpha}$ é uma álgebra de Boole, de forma que se $\beta<\alpha$ tem-se $A_{\beta} \subseteq A_{\alpha}$ então $A=\bigcup_{\alpha<\gamma} A_{\alpha}$ é uma álgebra de Boole.

Demonstração. Seja $a \in A$. Então, existe $\alpha<\gamma$ tal que $a \in A_{\alpha}$. Como $A_{\alpha}$ é uma álgebra de Boole, temos que $-a \in A_{\alpha} \subseteq A$. Sejam $a_{1}, a_{2} \in A$. Então, existem $\alpha_{1}, \alpha_{2}<\gamma$ tais que $a_{1} \in A_{\alpha_{1}}$ e $a_{2} \in A_{\alpha_{2}}$. Seja $\alpha=\max \left\{\alpha_{1}, \alpha_{2}\right\}$. Assim, temos que $a_{1}, a_{2} \in A_{\alpha}$. Logo,

$$
a_{1}+a_{2}, a_{1} \cdot a_{2} \in A_{\alpha} \subseteq A
$$

Além disso, $0,1 \in A_{0} \subseteq A$ e, portanto, $A$ é uma álgebra de Boole.

Com base neste exemplo, podemos construir, por indução transfinita, cadeias de álgebras de Boole, para obter álgebras de Boole com diferentes propriedades.

\subsection{2 Álgebras geradas}

Outro meio de obter álgebras de Boole é considerar álgebras geradas por subconjuntos de outras álgebras de Boole:

Definição 1.6. Seja $M$ um subconjunto de uma álgebra de Boole A. Então

$$
\langle M\rangle=\bigcap\{B \subseteq A: M \subseteq B, B \text { é subálgebra de } A\}
$$

é a subálgebra gerada por $M$.

\footnotetext{
${ }^{4} A$ é um corpo de conjuntos se é fechado por uniões e intersecções finitas, complementos e se $\emptyset \in A$.
} 


\section{ÁlGEBRAS DE BOOLE E ESPAÇOS COMPACTOS}

Tais tipos de álgebras têm características bem particulares, que facilitam o trabalho com elas. A principal delas, é que sabemos como (ou quais) são seus elementos. Vejamos, primeiramente, mais uma definição.

Definição 1.7. Seja A uma álgebra de Boole e $M \subseteq A$.

1. Um produto elementar sobre $M$ é um produto finito com fatores da forma $m$ ou $-m$, com $m \in M$.

2. Um elemento a $\in$ A está na forma normal sobre $M$ se èle é uma soma finita de produtos elementares sobre $M$, dois a dois disjuntos.

Temos então o seguinte teorema, que nos fornece quem são os elementos de uma álgebra gerada.

Teorema 1.8. A subálgebra gerada por $M \subseteq A$ contém exatamente os elementos de $A$ representáveis na forma normal sobre $M$.

Demonstração. Indicamos [Kopp], Proposição 4.4, página 51.

Tendo quais são os elementos de uma álgebra gerada, podemos contá-los, controlando a cardinalidade da álgebra, que nos será útil depois:

Corolário 1.9. Se $\omega \leq \alpha<2^{\omega}$ e A é uma álgebra de Boole gerada por $\alpha$ elementos, então $|A| \leq|\alpha|$.

Demonstração. Seja $A=\left\langle\left\{a_{\beta}: \beta<\alpha\right\}\right\rangle$. Pelo Teorema 1.8, temos que

$$
|A|=\mid\left\{\sum_{i=1}^{n}\left(\prod_{j_{i}=1}^{k_{i}}\left(A_{i, j_{i}}\right)\right): \text { cada } A_{i, j_{i}}=a_{\beta} \text { ou } A_{i, j_{i}}=-a_{\beta}, \text { para algum } \beta<\alpha\right\} \mid .
$$

Mas

$$
\mid\left\{\prod_{j_{i}=1}^{k_{i}}\left(A_{i, j_{i}}\right): \text { cada } A_{i, j_{i}}=a_{\beta} \text { ou } A_{i, j_{i}}=-a_{\beta}, \text { para algum } \beta<\alpha\right\}|\leq| \alpha^{<\omega}|=| \alpha \mid .
$$

Daí,

$$
\mid\left\{\sum_{i=1}^{n}\left(\prod_{j_{i}=1}^{k_{i}}\left(A_{i, j_{i}}\right)\right): \text { cada } A_{i, j_{i}}=a_{\beta} \text { ou } A_{i, j_{i}}=-a_{\beta} \text {, para algum } \beta<\alpha\right\}|\leq| \alpha^{<\omega}|=| \alpha \mid .
$$

Portanto, $|A| \leq|\alpha|$. 
O resultado a seguir será útil no Capítulo 7, onde precisamos saber como são alguns elementos de uma certa álgebra:

Corolário 1.10. Seja $B$ uma subálgebra de uma álgebra de Boole $A$ e a $\in A \backslash B$. Daí, se $b \in\langle B \cup\{a\}\rangle$, entẫo existem $E, F, G \in B \operatorname{com} E \cdot F=F \cdot G=E \cdot G=0$ tais que

$$
b=(E \cdot a)+(F \cdot(-a))+G .
$$

Demonstração. Seja $b \in\langle B \cup\{a\}\rangle$. Pelo teorema anterior, $b=\sum_{i=1}^{n}\left(\prod_{j_{i}=1}^{k_{i}}\left(C_{i, j_{i}}\right)\right)$, onde para cada $C_{i, j_{i}} \in B$, ou $C_{i, j_{i}}=a$ ou $C_{i, j_{i}}=-a$. Para cada $i$ fixo, considere $\prod_{j_{i}=1}^{k_{i}}\left(C_{i, j_{i}}\right)$. Assim, três coisas podem ocorrer: $C_{i, j_{i}}=a$ para algum $j_{i}$, ou $C_{i, j_{i}}=-a$ para algum $j_{i}$, ou $C_{i, j_{i}} \in B$ para todo $j_{i}$. (É claro que para cada $i$ ocorre apenas um dos casos.) Para o primeiro caso, escreva

$$
\prod_{j_{i}=1}^{k_{i}}\left(C_{i, j_{i}}\right)=\left(\prod_{j_{i}=1}^{k_{i}}\left\{C_{i, j_{i}}: C_{i, j_{i}} \neq a\right\}\right) \cdot a
$$

e defina $E_{i}=\prod_{j_{i}=1}^{k_{i}}\left\{C_{i, j_{i}}: C_{i, j_{i}} \neq a\right\}$ para tais $i$ 's. Para o segundo caso, escreva

$$
\prod_{j_{i}=1}^{k_{i}}\left(C_{i, j_{i}}\right)=\left(\prod_{j_{i}=1}^{k_{i}}\left\{C_{i, j_{i}}: C_{i, j_{i}} \neq(-a)\right\}\right) \cdot(-a)
$$

e defina $F_{i}=\prod_{j_{i}=1}^{k_{i}}\left\{C_{i, j_{i}}: C_{i, j_{i}} \neq(-a)\right\}$ para estes $i$ 's. Para o terceiro caso, apenas defina $G_{i}=\prod_{j_{i}=1}^{k_{i}} C_{i, j_{i}}$. Definimos, então,

$$
\begin{aligned}
& E=\sum\left\{E_{i}: \text { ocorre o primeiro caso para } i\right\} \\
& F=\sum\left\{F_{i}: \text { ocorre o segundo caso para } i\right\} \\
& G=\sum\left\{G_{i}: \text { ocorre o terceiro caso para } i\right\} .
\end{aligned}
$$

É claro que $E, F, G \in B$ e que $E \cdot F=F \cdot G=E \cdot G=0$. Por construção temos que

$$
b=(E \cdot a)+(F \cdot(-a))+G
$$

O próximo resultado nos mostra como se combinam os métodos de obter álgebras através de álgebras geradas e através de cadeias de álgebras. 


\section{ÁlGEBRAS DE BOOLE E ESPAÇOS COMPACTOS}

Lema 1.11. Seja A uma álgebra de Boole e $\left(a_{\alpha}\right)_{\alpha<2^{\omega}}$ uma seqüência de elementos de $A$. Se definimos, para cada $\alpha<2^{\omega}, A_{\alpha}=\left\langle\left\{a_{\beta}: \beta<\alpha\right\}\right\rangle$, temos que para cada $\alpha<2^{\omega}$ ordinal limite, $A_{\alpha}=\bigcup_{\beta<\alpha} A_{\beta}$.

Demonstração. Seja $\alpha<2^{\omega}$ um ordinal limite. É claro que se $\beta_{1}<\beta_{2}<2^{\omega}$ então $A_{\beta_{1}} \subseteq A_{\beta_{2}}$, pela definição. Então temos que $\bigcup_{\beta<\alpha} A_{\beta}$ é uma álgebra de Boole. Por um lado, para todo $\beta<\alpha$, temos que $a_{\beta} \in A_{\beta+1} \subseteq \bigcup_{\beta<\alpha} A_{\beta}$, pois $\alpha$ é limite. Logo, pela definição de subálgebra gerada, temos que $A_{\alpha} \subseteq \bigcup_{\beta<\alpha} A_{\beta}$. Por outro lado, dado $a \in \bigcup_{\beta<\alpha} A_{\beta}$, existe $\beta<\alpha$ tal que $a \in A_{\beta}$. Mas $A_{\beta} \subseteq A_{\alpha}$. Logo, $a \in A_{\alpha}$.

\subsubsection{Filtros e ultrafiltros}

Já temos agora uma noção de como obter álgebras de Boole. Na Seção 1.3, mostraremos como as álgebras de Boole se relacionam com os espaços compactos. Para isto, precisamos estudar um pouco sobre um interessante tipo de subconjunto de uma álgebra de Boole: os filtros.

Definição 1.12. Dizemos que $\mathcal{F} \subseteq A$ é um filtro em $A$ se satisfaz as seguintes condições:

1. $0 \notin \mathcal{F}$;

2. $1 \in \mathcal{F}$;

3. se $a, b \in \mathcal{F}$, então $a \cdot b \in \mathcal{F}$;

4. se $a \leq b$ e $a \in \mathcal{F}$, então $b \in \mathcal{F}$.

Definição 1.13. Dizemos que um filtro $\mathcal{F} \subseteq A$ é:

- um ultrafiltro se para todo $a \in A$ temos que $a \in \mathcal{F}$ ou $-a \in \mathcal{F}$;

- um filtro maximal se é um filtro e é maximal com respeito a esta propriedade.

O próximo resultado mostra que estas duas definições são equivalentes e permite que utilizemos qualquer uma delas ao trabalhar com ultrafiltros:

Proposição 1.14. Dados uma álgebra de Boole $A$ e um filtro $\mathcal{F}$ em $A$, as seguintes afirmaçôes são equivalentes: 
(a) $\mathcal{F}$ é um ultrafiltro;

(b) $\mathcal{F}$ é um filtro maximal.

Demonstração. (a) $\Rightarrow(\mathrm{b})$ : suponha que existe $a \in A \backslash \mathcal{F}$ tal que $\mathcal{F} \cup\{a\}$ é filtro. Como $a \notin \mathcal{F}$ temos que $-a \in \mathcal{F}$, pois $\mathcal{F}$ é ultrafiltro. Daí, temos que $a,-a \in \mathcal{F} \cup\{a\}$ e, portanto, $0=a \cdot(-a) \in \mathcal{F} \cup\{a\}$, uma contradição, pois $\mathcal{F} \cup\{a\}$ é filtro.

(b) $\Rightarrow($ a): suponha $\mathcal{F}$ uma filtro maximal e suponha, por absurdo, que existe $a \in A$ tal que $a,-a \notin \mathcal{F}$. Considere

$$
\mathcal{F}_{1}=\left\{b \in A: \exists e_{1}, \ldots, e_{n} \in \mathcal{F} \cup\{a\}, e_{1} \cdots e_{n} \leq b\right\},
$$

e $\mathcal{F}_{2}$ o análogo para $-a$. É fácil ver que $\mathcal{F}_{i}$ satisfaz 2,3 e 4 da definição de filtro, para $i=1,2$.

Vejamos que $\mathcal{F}_{1}$ ou $\mathcal{F}_{2}$ é filtro: senão, existem $e_{1}, \ldots, e_{n}, e_{1}^{\prime}, \ldots, e_{k}^{\prime} \in \mathcal{F}$ tais que $e_{1} \cdots e_{n} \cdot a=0$ e $e_{1}^{\prime} \cdots \cdot e_{k}^{\prime} \cdot(-a)=0$. Daí, $e_{1} \cdots e_{n} \leq-a$ e $e_{1}^{\prime} \cdots \cdot e_{k}^{\prime} \leq a$. Logo, $e_{1} \cdots e_{n} \cdot e_{1}^{\prime} \cdots e_{k}^{\prime}=0$, contradizendo que $\mathcal{F}$ é filtro. Logo, ou $\mathcal{F}_{1}$ ou $\mathcal{F}_{2}$ é um filtro que estende $\mathcal{F}$ : um absurdo, pois $\mathcal{F}$ é maximal.

Logo, ou $a \in \mathcal{F}$ ou $-a \in \mathcal{F}$ e, assim, $\mathcal{F}$ é um ultrafiltro.

Denotemos por $U l t(A)$ o conjunto dos ultrafiltros em $A$.

Definição 1.15. Dada uma álgebra de Boole $A$, dizemos que uma subfamília $\mathcal{C}$ de $A$ tem a propriedade de intersecção finita (usaremos p.i.f., no que segue) se para todos $a_{1}, \ldots, a_{n} \in \mathcal{C}$ temos que $a_{1} \cdots a_{n} \neq 0$.

A seguir, temos o mais importante meio de obter ultrafiltros: estendendo famílias com p.i.f.

Proposição 1.16. Um subconjunto de uma álgebra de Boole está incluído em um ultrafiltro se e somente se tem p.i.f.

Demonstração. Seja $A$ uma álgebra de Boole e $\mathcal{C} \subseteq A$. Se existe um ultrafiltro $p$ de $A$ tal que $\mathcal{C} \subseteq p$, como $p$ é fechado por intersecções finitas e não contém o 0 , segue que $\mathcal{C}$ tem a p.i.f. Por outro lado, se $\mathcal{C}$ tem a p.i.f., temos que

$$
p_{0}=\left\{a \in A: \exists e_{1}, \ldots, e_{n} \in \mathcal{C}, e_{1} \cdots e_{n} \leq a\right\}
$$




\section{ÁlGEBRAS DE BOOLE E ESPAÇOS COMPACTOS}

é um filtro. Considere

$$
\Phi=\left\{p \in U l t(A): p_{0} \subseteq p\right\}
$$

com a ordem definida por $p_{1} \leq p_{2} \Leftrightarrow p_{1} \subseteq p_{2}$, para $p_{1}, p_{2} \in \Phi$.

Temos que $\Phi$ é parcialmente ordenado (claro) e toda cadeia tem majorante (se $\mathcal{C}$ é uma cadeia, $\bigcup \mathcal{C} \in \Phi$ é um majorante). Então, pelo Lema de Zorn, existe $p \in \Phi$ elemento maximal. Logo, $p$ é um filtro maximal e $\mathcal{C} \subseteq p$.

\subsubsection{Homomorfismos}

Temos até aqui diversos resultados sobre álgebras de Boole. Queremos passar agora ao estudo de funções entre álgebras de Boole que preservam sua estrutura: os homomorfismos.

Definição 1.17. Sejam $A, B$ álgebras de Boole. Dizemos que $h: A \rightarrow B$ é um homomorfismo se para cada $a_{1}, a_{2} \in A$ tem-se que:

- $h\left(a_{1}+a_{2}\right)=h\left(a_{1}\right)+h\left(a_{2}\right)$;

- $h\left(a_{1} \cdot a_{2}\right)=h\left(a_{1}\right) \cdot h\left(a_{2}\right)$;

- $h\left(-a_{1}\right)=-h\left(a_{1}\right)$;

- $h\left(0_{A}\right)=0_{B}$;

- $h\left(1_{A}\right)=1_{B}$.

Dizemos que um homomorfismo hé um

(a) monomorfismo se é injetor;

(b) epimorfismo se é sobrejetor;

(c) isomorfismo se é bijetor.

Dizemos ainda que uma álgebra de Boole $B$ é imagem homomorfa de $A$ se existe um homomorfismo de $A$ sobre $B$ e duas álgebras de Boole são isomorfas se existe um isomorfismo entre elas. 
Para entender finalmente o último método para obter álgebras de Boole (quociente), precisamos de algumas definições:

Definição 1.18. Seja A uma álgebra de Boole. Dados $a, b \in A$, definimos a diferença simétrica entre a e $b$, e denotamos por $a \Delta b$, como o elemento $(a-b)+(b-a)$.

Definição 1.19. Seja A uma álgebra de Boole. Dizemos que $I \subseteq A$ é um ideal em $A$ se

- $0 \in I$;

- se $a \in I$ e $b \leq a$, então $b \in I$;

- se $a, b \in I$, então $a+b \in I$.

Além disso, dado $I \subseteq A$ um ideal, temos que a relação $\sim_{I}$ em $A$ dada por $a \sim_{I} b$ se $a \Delta b \in I$ é uma relação de equivalência. Daí, denotamos por $[a]=\left\{b \in A: a \sim_{I} b\right\} a$ classe de equivalência de a e denotamos por $A / I$ a álgebra de Boole quociente das classes de equivalência de elementos de A, com as seguintes operaçôes:

- $[a]+[b]=[a+b]$;

- $[a] \cdot[b]=[a \cdot b]$;

$-[a]=[-a]$

- $0=[0]$

- $1=[1]$

Mais ainda, dada uma álgebra de Boole $A$ e $I$ um ideal em $A$, temos que $h: A \rightarrow A / I$ dado por $h(a)=[a]$ é um epimorfismo. Porém, não faremos tal demonstração aqui.

Exemplo 1.20. Dado um homomorfismo sobrejetor $h: A \rightarrow B$, temos que $\operatorname{Ker}(h)$ é um ideal em $A$.

O teorema que segue permite que obtenhamos isomorfismos a partir de homomorfismos:

Teorema 1.21 (do homomorfismo). Seja $h: A \rightarrow B$ um homomorfismo sobrejetor de álgebras de Boole. Então, existe um isomorfismo $i: B \rightarrow A / \operatorname{Ker}(h)$. 
Demonstrą̧ão. Considere $i: B \rightarrow A / \operatorname{Ker}(h)$ dado por $i(h(a))=[a]$. Temos que $i$ está bem definido, pois se $h(a)=h\left(a^{\prime}\right)$, então $h(a) \Delta h\left(a^{\prime}\right)=0$ e daí, $h\left(a \Delta a^{\prime}\right)=0$. Logo, $a \Delta a^{\prime} \in \operatorname{Ker}(h)$ e, portanto, $[a]=\left[a^{\prime}\right]$.

Vejamos que $i$ é um homomorfismo. Temos que

$$
i\left(h(a)+h\left(a^{\prime}\right)\right)=i\left(h\left(a+a^{\prime}\right)\right)=\left[a+a^{\prime}\right]=[a]+\left[a^{\prime}\right]=i(h(a))+i\left(h\left(a^{\prime}\right)\right) .
$$

Para as demais operações, a demonstração é análoga. Assim, $i$ é um homomorfismo. É claro que $i$ é sobrejetor.

Vejamos que é injetor: sejam $a, a^{\prime} \in A$ tais que $i(h(a))=i\left(h\left(a^{\prime}\right)\right)$. Daí, $[a]=\left[a^{\prime}\right]$ e portanto, $\left[a \Delta a^{\prime}\right]=[a] \Delta\left[a^{\prime}\right]=0$. Disto, segue que $a \Delta a^{\prime} \in \operatorname{Ker}(h)$ e então $h(a) \Delta h\left(a^{\prime}\right)=$ $h\left(a \Delta a^{\prime}\right)=0$. Logo, $h(a)=h\left(a^{\prime}\right)$.

Ao contrário do Teorema do homomorfismo, queremos agora estender funções entre álgebras de Boole a homomorfismos. Isto não pode ser feito sempre. Vejamos quais resultados temos:

Teorema 1.22 (Primeiro critério de extensão de Sikorski). Sejam A, $B$ álgebras de Boole, $X \subseteq A$ e $\Phi: X \rightarrow B$. Temos que existe um homomorfismo $h:\langle X\rangle \rightarrow B$ que estende $\Phi$ se, e somente se,

$$
\forall F \subseteq X \text { finito, } \quad \forall e \in\{-1,1\}^{F} \quad \prod_{x \in F} e(x) x=0 \rightarrow \prod_{x \in F} e(x) \Phi(x)=0 .
$$

Além disso, se a volta também vale, o homomorfismo é um isomorfismo entre $\langle X\rangle$ e $h\langle[X]\rangle$. (Onde $-1 a=-a)$

Demonstração. Indicamos [Kopp], Teorema 5.5, página 67.

Teorema 1.23 (Segundo critério de extensão de Sikorski). Sejam $A, B, C$ álgebras de Boole. Suponha A subálgebra de $B$ e $h: A \rightarrow C$ um homomorfismo. Dado $b \in B \backslash A$, temos que existe $\tilde{h}:\langle A \cup\{b\}\rangle \rightarrow C$ um homomorfismo que estende $h$ se, e somente se, existe $c \in C$ tal que $\forall a, a^{\prime} \in A$

$$
a \leq b \leq a^{\prime} \rightarrow h(a) \leq c \leq h\left(a^{\prime}\right) .
$$

Além disso, se h é injetor, então $\tilde{h}$ é injetor se, e somente se, a volta de (*) também vale. 
Demonstração. Suponhamos, primeiramente que existe $\tilde{h}$ como no enunciado. Considere $c=\tilde{h}(b)$ e temos que se $a, a^{\prime} \in A$ são tais que $a \leq b \leq a^{\prime}$, então $\tilde{h}(a) \leq \tilde{h}(b) \leq \tilde{h}\left(a^{\prime}\right)$ e, portanto, $h(a) \leq c \leq h\left(a^{\prime}\right)$.

Reciprocamente, suponha que existe $c$ como no enunciado. Defina $\varphi: A \cup\{b\} \rightarrow C$ por $\varphi(a)=h(a)$, para todo $a \in A$ e $\varphi(b)=c$. Considere $F \subseteq A \cup\{b\}$ finito e $e \in\{-1,1\}^{F}$.

Se $b \notin F$, então $\varphi(x)=h(x)$ para todo $x \in F$ e daí temos que

$$
\prod_{x \in F} e(x) x=0 \rightarrow \prod_{x \in F} e(x) h(x)=0
$$

Se $b \in F$ e $e(b)=-1$, então

$$
\prod_{x \in F} e(x) x=0 \rightarrow\left(\prod_{x \in F \backslash\{b\}} e(x) x\right) \cdot(-b)=0 .
$$

Seja $a=\prod_{x \in F \backslash\{b\}} e(x) x$. Temos que $a \in A$ e $a \leq b$. Portanto, por hipótese, $h(a) \leq c=$ $\varphi(b)$. Logo, $h(a) \cdot(-\varphi(b))=0$. Mas

$$
h(a)=h\left(\prod_{x \in F \backslash\{b\}} e(x) x\right)=\prod_{x \in F \backslash\{b\}} e(x) h(x)=\prod_{x \in F \backslash\{b\}} e(x) \varphi(x) .
$$

Portanto, $\prod_{x \in F} e(x) \varphi(x)=0$.

Se $b \in F$ e $e(b)=1$, a demonstração é análoga ao caso anterior.

Assim, pelo 1.22 , existe $\tilde{h}:\langle A \cup\{b\}\rangle \rightarrow C$ estendendo $h$.

Suponhamos agora $h$ injetor e que a volta também vale. Suponha então $a \in\langle A \cup\{b\}\rangle$ tal que $\tilde{h}(a)=0$. Sabemos, pelo Corolário 1.10 que existem $E, F, G \in A \operatorname{com} E \cdot F=$ $F \cdot G=E \cdot G=0$ tais que $a=(E \cdot b)+(F \cdot(-b))+G$. Daí, temos que

$$
0=\tilde{h}(a)=(h(E) \cdot \tilde{h}(b))+(h(F) \cdot \tilde{h}(-b))+h(G)=(h(E) \cdot c)+(h(F) \cdot-c)+h(G) .
$$

Logo, $h(E) \cdot c=h(F) \cdot-c=h(G)=0$ e, como $h$ é monomorfismo, $G=0$. Além disso, $h(F) \leq c \leq-h(E)$. Como vale a volta, temos que $F \leq b \leq-E$ e, portanto, $E \cdot b=F \cdot-b=0$. Portanto, $a=0$ e assim, $\tilde{h}$ é injetor.

Reciprocamente, suponha $\tilde{h}$ injetor e que a volta não vale, isto é, que existe, por exemplo, $a \in A$ tal que $h(a) \leq c$ mas $a \not \leq b$. Daí, $a \cdot(-b)>0$. Como $\tilde{h}$ é injetor, $h(a) \cdot(-c)>0$, contradizendo que $h(a) \leq c$. 


\subsection{5 Álgebras completas}

Definição 1.24. Seja $A$ uma álgebra de Boole e $M \subseteq A$. Dizemos que a $\in A$ é o supremo de $M$ (e, neste caso, denotamos a por $\sum M$ ) se para todo $m \in M, m \leq a e$ para todo $b \in A$ que tem esta mesma propriedade, $a \leq b$. Dizemos que $A$ é uma álgebra de Boole completa se para todo $M \subseteq A$ existe o $\sum M$. Dizemos que $A$ é uma álgebra de Boole $\sigma$-completa se satisfaz esta condição para todo $M$ enumerável.

Dizemos, analogamente, que $a \in A$ é o ínfimo de $M$ (e, neste caso, denotamos a por $\left.\prod M\right)$ se para todo $m \in M, a \leq m$ e para todo $b \in A$ que tem esta mesma propriedade, $b \leq a$.

Notemos que se $M=\{a, b\} \subseteq A$, então $\sum M=a+b$. Além disso, podemos garantir que os ínfimos têm propriedades análogas às dos supremos, uma vez que são duais ${ }^{5}$.

O estudo deste tipo de álgebra se faz necessário, uma vez que tem boas propriedades. Vejamos um exemplo que elucida isto:

Exemplo 1.25. Seja $X$ um conjunto. $\wp(X)$ é uma álgebra de Boole completa.

Demonstração. Seja $M \subseteq \wp(X)$. Tome $Y=\bigcup M \in \wp(X)$. Temos que para todo $m \in M$, $m \subseteq Y$ e se $Z \in \wp(X)$ e para todo $m \in M$ tem-se que $m \subseteq Z$, então, $Y=\bigcup M \subseteq Z$. Logo, $Y=\sum M$ e, portanto, $\wp(X)$ é completa.

Vejamos então alguns resultados:

Definição 1.26. Seja $A$ uma álgebra de Boole. Dizemos que $M \subseteq A$ é uma anticadeia em $A$ se, para todos $a_{1}, a_{2} \in M, a_{1} \cdot a_{2}=0$.

Convém notar que no caso de corpos de conjuntos, uma anticadeia é uma coleção de conjuntos dois a dois disjuntos.

Proposição 1.27. Uma álgebra de Boole é completa se, e somente se, toda anticadeia tem supremo.

\footnotetext{
${ }^{5}$ Veja [Kopp], Teorema 1.13, página 13.
} 
Demonstração. A ida é clara. Vejamos a volta: seja $M \subseteq A$ queremos mostrar que existe $\sum M$. Seja $B$ uma anticadeia maximal em $C:=\{b \in A: \exists m \in M b \leq m\}$ Temos que tal cadeia existe, pelo Lema de Zorn. Seja $c:=\sum B$. Vamos mostrar que $c=\sum M$. Suponha que existe $m \in M$ tal que $m \not \leq c$, então $m-c \neq 0$. Como $(m-c) c=m(-c) c=0$, temos que para todo $b \in B,(m-c) b=0$, pois $b \leq c$. Então $B \cup\{m-c\}$ é uma anticadeia em $C$. E note que $m-c \neq b$ para todo $b \in B$, pois senão teríamos que $0=(m-c) b=(m-c)(m-c)=(m-c) \neq 0$, uma contradição. Assim, $B \cup\{m-c\}$ é uma anticadeia em $C$ que contém $B$ propriamente. Contradição com a maximalidade de $B$. Seja $d \in A$ tal que $m \leq d$ para todo $m \in M$. Então $b \leq d$ para todo $b \in B$, ou seja, $\sum B=c \leq d$. Assim, temos que $c=\sum M$.

Teorema 1.28 (da Extensão de Sikorski). Sejam $A, B, C$ álgebras de Boole e $h$ : $A \rightarrow C$ um homomorfismo. Se $A$ é subálgebra de $B$ e $C$ é completa, então existe um homomorfismo $\tilde{h}: B \rightarrow C$ tal que $\left.\tilde{h}\right|_{A}=h$.

Demonstração. Considere o conjunto

$\left\{\left(A^{\prime}, h^{\prime}\right): A^{\prime}\right.$ é subálgebra de $B, A \subseteq A^{\prime}, h^{\prime}: A^{\prime} \rightarrow C$ é homomorfismo que estende $\left.h\right\}$,

com a ordem $\left(A^{\prime}, h^{\prime}\right) \leq\left(A^{\prime \prime}, h^{\prime \prime}\right)$ se, e somente se, $A^{\prime} \subseteq A^{\prime \prime}$ e $h^{\prime} \subseteq h^{\prime \prime}$.

Note que se $\mathcal{F}$ é uma cadeia, então $\left(\bigcup\left\{A^{\prime}: \exists h^{\prime}\left(A^{\prime}, h^{\prime}\right) \in \mathcal{F}\right\}, \bigcup\left\{h^{\prime}: \exists A^{\prime}\left(A^{\prime}, h^{\prime}\right) \in\right.\right.$ $\mathcal{F}\}$ ) é um limitante superior da cadeia, uma vez que união de subálgebras de $B$ é uma subálgebra de $B$ e união crescente de homomorfismos é um homomorfismo.

Pelo Lema de Zorn, existe $\left(A^{\prime}, h^{\prime}\right)$ maximal. É claro que $h^{\prime}$ é extensão de $h$. Além disso, temos que $h^{\prime}$ está definido em $B$, pois senão, existiria $b \in B \backslash A^{\prime}$ e pelo Teorema $1.23, h^{\prime}$ poderia ser estendida a $\left\langle A^{\prime},\{b\}\right\rangle$ e $\left(A^{\prime}, h^{\prime}\right)$ não seria maximal.

\subsection{Espaços topológicos}

O objetivo desta seção é lembrar um pouco sobre propriedades de espaços topológicos que consideraremos. Queremos também contextualizar os espaços que pretendemos estudar, dentro da topologia geral.

Lembremos primeiramente algumas definições: 


\section{ÁLGEBRAS DE BOOLE E ESPAÇOS COMPACTOS}

Definição 1.29. Dizemos que um espaço topológico $K$ é um:

(a) espaço Hausdorff se para todos $x, y \in K \operatorname{com} x \neq y$, existem abertos $U$ e $V$ tais que $x \in U, y \in V$ e $U \cap V=\emptyset$;

(b) espaço compacto se para todo $\mathcal{C}$ recobrimento aberto de $K$ existe um subrecobrimento finito;

(c) espaço zero-dimensional se possui uma base de conjuntos abertos-fechados para a topologia;

(d) espaço metrizável se existe uma métrica $d: K \times K \rightarrow \mathbb{R}$ tal que a topologia induzida pela métrica é a topologia de $K$.

Trabalharemos, nos Capítulos 4, 5, 6 e 7 com espaços compactos Hausdorff. Assim, lembramos ainda alguns resultados importantes relacionados a tais tipos de espaços topológicos.

Proposição 1.30. Um espaço topológico $K$ é compacto se, e somente se, para toda família $\mathcal{F}$ de fechados com a propriedade de intersecção finita ${ }^{6}$ tem-se que $\bigcap \mathcal{F} \neq \emptyset$.

Demonstração. $(\Rightarrow)$ : suponha $K$ compacto e $\mathcal{F}$ uma família de fechados tal que $\bigcap \mathcal{F}=\emptyset$. Então, $\mathcal{C}=\{K \backslash F: F \in \mathcal{F}\}$ é um recobrimento aberto de $K$. Como $K$ é compacto, existem $F_{1}, \ldots F_{n} \in \mathcal{F}$ tais que $K \subseteq\left(K \backslash F_{1}\right) \cup \cdots \cup\left(K \backslash F_{n}\right)$. Logo, $F_{1} \cap \cdots \cap F_{n}=\emptyset \mathrm{e}$, portanto, $\mathcal{F}$ não tem p.i.f.

$(\Leftarrow)$ : suponha, por absurdo, que $K$ não é compacto. Seja $\mathcal{C}$ um recobrimento aberto de $K$ que não possui subrecobrimento finito. Então, $\mathcal{F}=\{K \backslash C: C \in \mathcal{C}\}$ é uma família de fechados. Vejamos que $\mathcal{F}$ tem p.i.f.: sejam $C_{1}, \ldots, C_{n} \in \mathcal{C}$. Temos que existe $x \in K \backslash\left(C_{1} \cup \cdots \cup C_{n}\right)$, pois senão, $\left\{C_{1}, \ldots, C_{n}\right\}$ seria um subrecobrimento finito. Logo, $x \in\left(K \backslash C_{1}\right) \cap \cdots \cap\left(K \backslash C_{n}\right)$.

Como dissemos, trabalharemos com espaços compactos Hausdorff. Tais espaços são espaços bem particulares dentro da topologia geral. Para exemplificar isto, mostremos que espaços compactos Hausdorff são normais:

\footnotetext{
${ }^{6}$ Lembramos que definimos p.i.f. para álgebras de Boole. A família dos subconjuntos fechados de um espaço topológico não é, necessariamente, uma álgebra de Boole. Porém, definimos a p.i.f. analogamente, isto é, uma família de fechados $\mathcal{F}$ tem p.i.f. se para quaisquer $F_{1}, \ldots, F_{n} \in \mathcal{F}$, tem-se que $F_{1} \cap \cdots \cap F_{n} \neq \emptyset$.
} 
Definição 1.31. Seja $K$ um espaço topológico Hausdorff. Dizemos que $K$ é um espaço normal $^{7}$ se para quaisquer $E, F \subseteq K$ fechados disjuntos, existem $U, V \subseteq K$ abertos disjuntos tais que $E \subseteq U$ e $F \subseteq V$.

Proposição 1.32. Seja K um espaço topológico compacto Hausdorff. Então temos que $K$ é normal.

Demonstração. Sejam $E, F \subseteq K$ fechados disjuntos. Fixemos, primeiramente $x \in E$ qualquer. Para cada $y \in F$, temos que $x \neq y$ e, como $K$ é Hausdorff, existem $V_{y}, W_{y}$ abertos disjuntos tais que $x \in V_{y}$ e $y \in W_{y}$. Temos que $F \subseteq \bigcup\left\{W_{y}: y \in F\right\}$. Como $F$ é fechado num compacto, temos que é compacto e, portanto, existe $F^{*} \subseteq F$ finito tal que $F \subseteq \bigcup\left\{W_{y}: y \in F^{*}\right\}$. Defina $U_{x}=\bigcup\left\{W_{y}: y \in F^{*}\right\}$ e $V_{x}=\bigcap\left\{V_{y}: y \in F^{*}\right\}$. Temos que $V_{x}$ e $U_{x}$ são abertos disjuntos, $x \in V_{x}$ e $F \subseteq U_{x}$.

Temos então, que $E \subseteq \bigcup\left\{U_{x}: x \in E\right\}$. Como $E$ é fechado num compacto, temos que é compacto e, portanto, existe $E^{*} \subseteq E$ finito tal que $E \subseteq \bigcup\left\{U_{x}: x \in E^{*}\right\}$. Defina $U=\bigcup\left\{U_{x}: x \in E^{*}\right\}$ e $V=\bigcap\left\{V_{x}: x \in E^{*}\right\}$. Temos que $V$ e $U$ são abertos disjuntos, $E \subseteq V$ e $F \subseteq U$. Daí segue que $K$ é normal.

Para melhor contextualizar os espaços com os quais trabalharemos dentro da topologia, vamos agora compará-los aos espaços métricos. Neste sentido, temos o seguinte:

Definição 1.33. Seja $K$ um espaço topológico. Definimos a densidade de $K$ (e denotamos por $d(K))$ por

$$
d(K)=\min \{|D|: D \subseteq K \text { é denso em } K\}+\omega .
$$

Dizemos que $K$ é um espaço separável se $d(K)=\omega$.

Proposição 1.34. Seja $K$ um espaço métrico. Então temos que:

1. se $K$ é compacto, então $K$ tem base enumerável;

2. K tem base enumerável se, e somente se, $K$ é separável.

\footnotetext{
${ }^{7} \mathrm{Na}$ realidade, a definição de um espaço normal é um pouco mais fraca que esta. Porém, esta definição é equivalente à definição usual de espaços normais, para espaços Hausdorff. Como trabalharemos apenas com tais espaços, achamos mais natural definir normalidade desta forma.
} 
Demonstração. 1. Para cada $n \in \mathbb{N}$, existe $A_{n} \subseteq K$ finito tal que $K=\bigcup\left\{B\left(x, \frac{1}{n}\right)\right.$ : $\left.x \in A_{n}\right\}$. Considere

$$
\mathcal{B}=\left\{B\left(x, \frac{1}{n}\right): x \in A_{n} \text { e } n \in \mathbb{N}\right\} .
$$

É claro que $\mathcal{B}$ é enumerável. Vejamos que é base: seja $U \subseteq K$ um aberto e seja $x \in U$. Temos que existe $m \in \mathbb{N}$ tal que $B\left(x, \frac{1}{m}\right) \subseteq U$. Seja $y \in A_{m}$ tal que $y \in B\left(x, \frac{1}{2 m}\right)$. Temos que $x \in B\left(y, \frac{1}{2 m}\right) \subseteq B\left(x, \frac{1}{m}\right)$ e $B\left(y, \frac{1}{2 m}\right) \in \mathcal{B}$.

2. $(\Rightarrow)$ : seja $\mathcal{B}$ uma base enumerável para $K$. Para cada $U \in \mathcal{B}$, tomemos $x_{U} \in U$. Temos que $D=\left\{x_{U}: U \in \mathcal{B}\right\}$ é um denso enumerável. (Na verdade, esta implicação vale para todo espaço topológico.)

$(\Leftarrow)$ : seja $D=\left\{x_{n}: n \in \mathbb{N}\right\}$ um denso em $K$. Temos que

$$
\mathcal{B}=\left\{B\left(x_{n}, \frac{1}{k}\right): n, k \in \mathbb{N}\right\}
$$

é uma base enumerável para $K$.

O Teorema de Tychonoff (abaixo) pode ser utilizado para obter novos espaços compactos a partir de alguns dados.

Teorema 1.35 (de Tychonoff). Seja $\left(K_{i}, \mathcal{T}_{i}\right)_{i \in I}$ uma família de espaços topológicos não vazios e $\left(\Pi_{i \in I} K_{i}, \mathcal{T}\right)$, onde $\mathcal{T}$ é a topologia produto ${ }^{8} .\left(\Pi_{i \in I} K_{i}, \mathcal{T}\right)$ é compacto se, e somente se, $\left(K_{i}, \mathcal{T}_{i}\right)$ é compacto para todo $i \in I$.

Demonstração. Indicamos [Eng], Teorema 3.2.4, página 138.

Os espaços que consideraremos são, em particular, espaços normais, como já provamos. O teorema que segue é bastante famoso e permite que estendamos funções contínuas a valores reais de certos subconjuntos destes espaços ao espaço inteiro:

Teorema 1.36 (da Extensão de Tietze). Seja $K$ um espaço topológico normal. Se $M \subseteq K$ é um subconjunto fechado de $K$ e $f: M \rightarrow \mathbb{R}$ uma função contínua, então existe uma função contínua $\tilde{f}: K \rightarrow \mathbb{R}$ tal que $\left.\tilde{f}\right|_{M}=f$ e $\|\tilde{f}\|=\|f\|$.

\footnotetext{
${ }^{8}$ Lembramos que se $\left(K_{i}, \mathcal{T}_{i}\right)_{i \in I}$ é uma família de espaços topológicos, a topologia produto em $\Pi_{i \in I} K_{i}$ é aquela gerada por elementos da forma $\prod_{i \in I} U_{i}$, onde $\left|\left\{i \in I: U_{i} \neq K_{i}\right\}\right|<\omega$ e para todo $i \in I, U_{i}$ é um aberto em $K_{i}$.
} 
Demonstração. Indicamos [Eng], Teorema 2.1.8, página 69 .

Definamos um outro cardinal topológicos para entender o próximos resultado:

Definição 1.37. Seja $K$ um espaço topológico. Definimos o peso de $K$ (e denotamos por $p(K))$ por

$$
p(K)=\min \{|B|: B \text { é base para a topologia de } K\}+\omega .
$$

Para terminar esta seção, vejamos que o axioma de Martin implica que se um espaço topológico $K$ tem peso menor que $2^{\omega}$, então $K$ tem seqüências convergentes não triviais. No Capítulo 3 , veremos que neste caso, $C(K)$ tem uma cópia complementada de $c_{0}$. Esta informação será bastante útil ao estudarmos espaços de Grothedieck da forma $C(K)$. Para fazer este resultado, precisamos de um pouco sobre o axioma de Martin (MA): o axioma de Martin é um princípio conjuntístico consistente com $\mathrm{ZFC}^{9}$. Sabe-se que MA é consistente com $\neg \mathrm{CH}$, a negação da hipótese do contínuo $^{10} \mathrm{Na}$ verdade, mais do que isso, sabemos que MA é consistente com $2^{\omega}=\kappa$ para diversos cardinais $\kappa$ (os regulares, por exemplo).

Para entendermos seu enunciado, vejamos primeiramente a definição do cardinal $p$ : chamamos $p$ ao menor cardinal tal que

existe $\left(M_{\alpha}\right)_{\alpha<p} \subseteq \wp(\mathbb{N})$ com $\bigcap_{\alpha \in F} M_{\alpha}$ infinito para todo $F \subseteq p$ finito, e não existe $M \subseteq \mathbb{N}$ infinito, tal que $\left|M \backslash M_{\alpha}\right|<\infty$ para todo $\alpha<p$.

Isto significa que podemos de certa forma diagonalizar menos que $p$ subconjuntos de $\mathbb{N}$ que sejam finitamente compatíveis. Sabe-se que $\omega_{1} \leq p \leq 2^{\omega}$ e que MA implica ${ }^{11}$ que $p=2^{\omega}$. Mostremos então o que queremos:

Proposição 1.38. (MA) Todo espaço compacto Hausdorff infinito com peso ${ }^{12}$ menor que $2^{\omega}$ tem seqüência convergente não trivial.

\footnotetext{
${ }^{9} \mathrm{ZFC}$ é a teoria de Zermelo-Fraenkel com o axioma da escolha (AC).

${ }^{10} \mathrm{~A}$ hipótese do contínuo $(\mathrm{CH})$ diz que $2^{\omega}=\omega_{1}$, isto é, que a cardinalidade de $\wp(\mathbb{N})$ é o primeiro cardinal não enumerável.

${ }^{11}$ Veja [We].

${ }^{12}$ Convém notar que a demonstração deste resultado garante algo mais forte: todo espaço compacto Hausdorff infinito com caráter menor que $2^{\omega}$ tem uma seqüência convergente não trivial.
} 
Demonstração. Seja $\left\{x_{n}: n \in \mathbb{N}\right\}=X \subseteq K$, com $x_{n} \neq x_{m}$ se $n \neq m$. Como $K$ é compacto, existe $x \in K$ ponto de acumulação de $X$. Seja $\mathcal{B}$ uma base de vizinhanças para $x$ de cardinalidade menor que $2^{\omega}$. Para cada $B \in \mathcal{B}$, defina $X_{B}=\left\{n \in \mathbb{N}: x_{n} \in B\right\}$ e considere a família $\mathcal{A}=\left\{X_{B}: B \in \mathcal{B}\right\}$ de subconjuntos de $\mathbb{N}$.

Vejamos que $\mathcal{A}$ tem p.i.f.: sejam $B_{1}, \ldots B_{k} \in \mathcal{B}$. Como para todo $1 \leq l \leq k$ temos que $B_{l}$ é vizinhança de $x$, segue que $\bigcap_{1 \leq l \leq k} B_{l} \neq \emptyset$ é uma vizinhança de $x$. Como $\mathcal{B}$ é base de vizinhanças de $x$, existe $C \in \mathcal{B}$ tal que $C \subseteq \bigcap_{1 \leq l \leq k} B_{l}$. Daí,

$$
\bigcap_{1 \leq l \leq k} X_{B_{l}}=\left\{n \in \mathbb{N}: x_{n} \in \bigcap_{1 \leq l \leq k} B_{l}\right\} \supseteq\left\{n \in \mathbb{N}: x_{n} \in C\right\}=X_{C} \text { é infinito, }
$$

pois $x$ é ponto de acumulação de $X$.

Como $|\mathcal{B}|<2^{\omega}$, por MA, temos que existe $A \subseteq \mathbb{N}$ infinito tal que $\left|A \backslash X_{B}\right|$ é finito para todo $B \in \mathcal{B}$. Considere $A=\left\{n_{0}<n_{1}<n_{2}<\ldots\right\}$.

Vejamos que a seqüência $\left(x_{n_{k}}\right)_{k \in \mathbb{N}}$ converge para $x$ : seja $V$ uma vizinhança de $x$. Então existe $B \in \mathcal{B}$ tal que $x \in B \subseteq V$. Como $A \backslash X_{B}$ é finito, temos que existe $k_{0} \in \mathbb{N}$ tal que se $k \geq k_{0}$, então $x_{n_{k}} \in B$. Portanto, $x_{n_{k}} \rightarrow x$.

\subsection{A dualidade de Stone}

\subsubsection{O espaço de Stone de uma álgebra}

Finalmente temos todos os elementos necessários para entender a dualidade existente entre álgebras de Boole e espaços compactos Hausdorff. O primeiro passo da dualidade de Stone é o Teorema de Representação de Stone, que garante que, dada uma álgebra de Boole, podemos obter um espaço compacto Hausdorff e zero-dimensional a ela relacionado e vice-versa. Comecemos então entendendo qual será o espaço topológico associado a uma álgebra de Boole:

Definição 1.39. Seja A uma álgebra de Boole. Considere Ult(A) o conjunto dos ultrafiltros de A. Para cada $a \in A$, definimos o conjunto $a^{*}$ por

$$
a^{*}=\{p \in U l t(A): a \in p\} .
$$


Os conjuntos assim definidos formarão uma base para uma topologia em $\operatorname{Ult}(A)$. Além de ser uma base, será formado por subconjuntos abertos-fechados de $U l t(A)$. Tal propriedade seguirá diretamente do seguinte resultado:

Proposição 1.40. Seja A uma álgebra de Boole. Então, para todo $a, b \in A$ temos:

1. $U l t(A) \backslash a^{*}=(-a)^{*}$;

2. $a^{*} \cup b^{*}=(a+b)^{*}$;

3. $a^{*} \cap b^{*}=(a \cdot b)^{*}$.

Demonstração. 1. Seja $a \in A$. Tomemos $p \in U l t(A) \backslash a^{*}$. Então $a \notin p$ e, portanto, $-a \in p$. Logo, $p \in(-a)^{*}$. Por outro lado, tomemos $p \in(-a)^{*}$. Isto significa que $-a \in p$. Daí, $a \notin p$ e, portanto, $p \notin a^{*}$.

2. Sejam $a, b \in A$. Tomemos $u \in a^{*} \cup b^{*}$. Temos então que ou $u \in a^{*}$ ou $u \in b^{*}$ e daí, ou $a \in u$ ou $b \in u$. Como $a, b \leq a+b$, em qualquer dos casos teremos que $a+b \in u$ e portanto $u \in(a+b)^{*}$. Por outro lado, tomemos $u \in(a+b)^{*}$. Então $a+b \in u$. Além disso, como $u$ é ultrafiltro, temos que ou $a \in u$ ou $-a \in u$. Se $a \in u$, então $u \in a^{*} \subseteq a^{*} \cup b^{*}$. Senão, temos que $-a \in u$ e portanto, $(a+b) \cap(-a) \in u$. Mas $(a+b) \cap(-a) \leq b$ e como $u$ é filtro temos que $b \in u$. Logo $u \in b^{*} \subseteq\left(a^{*} \cup b^{*}\right)$ e segue a igualdade.

3. Sejam $a, b \in A$. Tomemos $u \in a^{*} \cap b^{*}$. Então temos que $a, b \in u$ e como $u$ é filtro, $a \cdot b \in u$. Portanto $u \in(a \cdot b)^{*}$. Por outro lado, tomemos $u \in(a \cdot b)^{*}$. Então $a \cdot b \in u$ e como $u$ é filtro e $a \cdot b \leq a, b$, segue que $a, b \in u$. Portanto $u \in a^{*}, b^{*}$ e segue a igualdade.

Definição 1.41. Um espaço topológico $X$ é dito um espaço booleano se é compacto Hausdorff e zero-dimensional.

Definição 1.42. Dada uma álgebra de Boole A, a topologia de Stone é a topologia sobre Ult $(A)$ gerada ${ }^{13}$ por $\left\{a^{*}: a \in A\right\}$. Ao conjunto Ult $(A)$ munido desta topologia chamamos o espaço de Stone de $A$ e denotamos por $S(A)$.

\footnotetext{
${ }^{13}$ Temos em [Eng], Proposição 1.2.1, página 20, condições suficientes para que uma família gere uma topologia. É fácil ver que esta família satisfaz tais condições.
} 


\section{ÁLGEBRAS DE BOOLE E ESPAÇOS COMPACTOS}

Vejamos então que o espaço assim obtido é um espaço booleano:

Teorema 1.43. Se A é uma álgebra de Boole, então seu espaço de Stone $S(A)$ é um espaço booleano.

Demonstração. Primeiramente, provemos que $S(A)$ é compacto. Seja $\mathcal{C}$ um recobrimento aberto de $S(A)$. Para todo $p \in S(A)$, existem $a_{p} \in A$ e $C_{p} \in \mathcal{C}$ tais que $p \in a_{p}^{*} \subseteq C_{p}$. Suponha por absurdo que para todo $a_{p_{1}}, \ldots, a_{p_{n}}$, existe $p \in S(A)$ tal que $p \notin a_{p_{1}}^{*} \cup \cdots \cup a_{p_{n}}^{*}$. Daí, o conjunto $\left\{S(A)-a_{p}^{*}: p \in S(A)\right\}=\left\{\left(-a_{p}\right)^{*}: p \in S(A)\right\}$ tem a p.i.f.: sejam $p_{1}, \ldots, p_{n} \in S(A)$. Temos que existe $p \in S(A)$ tal que $p \notin a_{p_{1}}^{*} \cup \cdots \cup a_{p_{n}}^{*}$. Daí, $p \in$ $\left(-a_{p_{1}}\right)^{*} \cap \cdots \cap\left(-a_{p_{n}}\right)^{*} \neq \emptyset$. Então o conjunto $\left\{-a_{p}: p \in S(A)\right\}$ tem a p.i.f.: sejam $p_{1}, \ldots, p_{n} \in S(A)$. Temos que existe $p \in\left(-a_{p_{1}}\right)^{*} \cap \cdots \cap\left(-a_{p_{n}}\right)^{*}$. Então $-a_{p_{1}}, \ldots,-a_{p_{n}} \in p$ e como $p$ é filtro, $\left(-a_{p_{1}}\right) \cdots \cdot\left(-a_{p_{n}}\right) \neq 0$. Assim, podemos estender esta família a um ultrafiltro, digamos, $p_{0} \in S(A)$. Daí temos que $-a_{p} \in p_{0}$, para todo $p \in S(A)$, em particular, $-a_{p_{o}} \in p_{0}$. Mas por hipótese temos que $p_{0} \in a_{p_{0}}^{*}$ e, portanto, $a_{p_{0}} \in p_{o}$. Contradição. Logo, existem $p_{1}, \ldots, p_{n} \in S(A)$ tal que

$$
S(A) \subseteq a_{p_{1}}^{*} \cup \cdots \cup a_{u_{n}}^{*} \subseteq C_{p_{1}} \cup \cdots \cup C_{p_{n}} .
$$

Portanto, $S(A)$ é compacto.

Provemos, então, que $S(A)$ é Hausdorff. Sejam $p_{1}, p_{2} \in S(A), p_{1} \neq p_{2}$. Seja $a \in A$ tal que $a \in p_{1}$ e $a \notin p_{2}$. Como $a \notin p_{2}$, temos que $-a \in p_{2}$. Considere, então, os abertos $a^{*}$ e $(-a)^{*}$. Temos que $p_{1} \in a^{*}$ e $p_{2} \in(-a)^{*}$. Vejamos que estes dois abertos são disjuntos: suponha, por absurdo, que existe $p \in(-a)^{*} \cap a^{*}$. Então $a \in p$ e $-a \in p$. Como $p$ é filtro, teríamos que $0 \in p$, o que não pode ocorrer. Portanto, $a^{*}$ e $(-a)^{*}$ são abertos disjuntos, $p_{1} \in a^{*}$ e $p_{2} \in(-a)^{*}$. Assim, $S(A)$ é Hausdorff.

Por fim, provemos que $S(A)$ é zero-dimensional. Como $S(A)-a^{*}=(-a)^{*}$ e $b^{*}$ é aberto, qualquer que seja $b \in A$, temos que a família $\mathcal{B}=\left\{a^{*}: a \in A\right\} \subseteq S(A)$ é uma família de conjuntos aberto-fechados em $S(A)$ e já sabemos que é base da topologia de $S(A)$. Logo, $S(A)$ é zero-dimensional.

\subsubsection{A representação de Stone}

Já sabemos que dada uma álgebra de Boole, temos um espaço booleano. Vejamos que a ligação entre estes dois objetos é muito forte: 
Definição 1.44. Seja $K$ um espaço booleano. Definimos a álgebra ${ }^{14}$ dos abertos-fechados de $K$ e denotamos por $C l o p(K)$ a seguinte subálgebra de $\wp(K)$ :

$$
C l o p(K)=\{a \subseteq K: a \text { é aberto-fechado }\}
$$

com as operações conjuntísticas.

Teorema 1.45 (da Representação de Stone). Toda álgebra de Boole é isomorfa à álgebra $C l o p(K)$, para algum espaço booleano $K$.

Demonstração. Seja $A$ uma álgebra de Boole e $K=S(A)$. O fato que a função $*$ que leva $a \in A$ em $a^{*} \in C l o p(S(A))$ é um homomorfismo de $A$ em $C l o p(K)$ segue da Proposição 1.40 e do fato que $0^{*}=\emptyset$.

Que é injetor, segue do fato que se $a \neq b$, então existe $u \in U l t(A)$ tal que $a \in u \mathrm{e}$ $b \notin u$ e portanto, $u \in a^{*}$ e $u \notin b^{*}$.

Vejamos que é sobrejetor: seja $U \in C l o p(S(A))$. Como $A$ é base, existe $\mathcal{V} \subseteq A$ tal que $U=\bigcup \mathcal{V}$. Como $U$ é fechado num compacto, temos que $U$ é compacto e daí, existem $V_{1}, \ldots, V_{n} \in \mathcal{V} \subseteq A$ tais que $U=V_{1} \cup \ldots V_{n} \in A$.

Para obter uma álgebra de Boole a partir de um espaço booleano, basta considerar o conjunto dos subconjuntos abertos-fechados em tal espaço. Vejamos que isto de fato acontece:

Teorema 1.46. Todo espaço topológico booleano é homeomorfo ao espaço de Stone de uma álgebra de Boole.

Demonstração. Seja $K$ um espaço booleano. Vejamos que $K$ é homeomorfo a $S(C l o p(K))$ : defina $f: K \rightarrow S(C l o p(K))$ por $f(x)=\{a \in C l o p(K): x \in a\}$. Vejamos primeiramente que $f$ está bem definida: é claro que $f(x)$ é filtro e é ultrafiltro, pois dado $a \in C l o p(K)$, ou $x \in a$ ou $x \in-a$.

Vejamos que $f$ é injetora: sejam $x, y \in K$. Como $K$ é Hausdorff, existem $U, V$ abertos disjuntos tais que $x \in U$ e $y \in V$. Usando a compacidade e a normalidade (Proposição 1.32) de $K$ segue que existe $a \in C l o p(K)$ tal que $x \in a$ e $y \notin a$. Daí, $a \in f(x)$ e $a \notin f(y)$. Logo, $f(x) \neq f(y)$.

\footnotetext{
${ }^{14} \mathbf{E}$ fácil ver que o conjunto assim definido é uma álgebra de Boole.
} 
Para mostrar que $f$ é sobrejetora, tomemos $u \subseteq C l o p(K)$ um ultrafiltro. Temos que $u$ é uma família de abertos com p.i.f., num compacto, logo, existe $x \in \bigcap u$. Daí, é fácil ver que $f(x)=u$.

Por fim, provemos que $f$ é contínua: seja $U \subseteq S(C l o p(K))$ um aberto básico. Daí, existe $a \in A$ tal que $U=a^{*}=\{u \in S(C l o p(K)): a \in u\}$. Temos que

$$
h^{-1}\left[a^{*}\right]=\{x \in K: x \in a\}=a, \text { que é aberto. }
$$

Como os espaços são compactos Hausdorff, temos que $f$ é um homeomorfismo.

Corolário 1.47. Sejam $A$ e $B$ álgebras de Boole. Então, $A$ e $B$ são isomorfas se, $e$ somente se, $S(A)$ e $S(B)$ são homeomorfos.

Corolário 1.48. Se A é isomorfa a Clop $(K)$ para um espaço $K$ booleano, então $K$ é homeomorfo a $S(A)$.

A partir destes corolários, por abuso de linguagem, diremos que $K$ é o espaço de Stone de uma álgebra de Boole $A$ se $K$ é homeomorfo a $S(A)$. Vejamos alguns exemplos:

Exemplo 1.49 (uma seqüência convergente com seu limite). Considere a álgebra de Boole

$$
\text { FinCofin }(\mathbb{N})=\{a \subseteq \mathbb{N}: \text { a é finito ou cofinito }\} .
$$

Temos que $S($ FinCofin $(\mathbb{N}))$ é uma seqüência convergente.

Demonstração. Pelo corolário anterior, basta mostrar que se $K=\left\{x_{n}: n \in \mathbb{N}\right\} \cup\{x\}$, onde cada $\left\{x_{n}\right\}$ é aberto-fechado e $x_{n}$ converge a $x$, então $C l o p(K)$ é isomorfo à álgebra $\operatorname{FinCofin}(\mathbb{N})$. Definimos $h: C l o p(K) \rightarrow \operatorname{FinCofin}(\mathbb{N})$ por

$$
h(a)=\left\{n \in \mathbb{N}: x_{n} \in a\right\} .
$$

$h$ está bem definido, pois se $a$ é aberto-fechado de $K$, temos que:

- se $x \in a$, então $\left\{n \in \mathbb{N}: x_{n} \notin a\right\}$ é finito e, portanto, $h(a)$ é cofinito;

- se $x \notin a$, então $x \in K \backslash a$ e daí, $\left\{n \in \mathbb{N}: x_{n} \in a\right\}=\left\{n \in \mathbb{N}: x_{n} \notin K \backslash a\right\}$ é finito e, portanto, $h(a)$ é finito. 
É fácil ver que $h$ é um homomorfismo.

Que $h$ é injetor, segue do fato que se $a \neq b \in C l o p(K)$, então existe $y \in K$ tal que $y \in a$ se, e somente se, $y \notin b$. Se $y=x_{n}$ para algum $n \in \mathbb{N}$, temos o resultado. Se $y=x$, então $y \in a$ se, e somente se, $a$ é cofinito e daí, segue que $a$ é cofinito se, e somente se, $b$ é finito. Portanto $h(a)$ é cofinito se, e somente se, $h(b)$ é finito. Logo, $h(a) \neq h(b)$.

Por fim, vejamos que $h$ é sobrejetor: isto segue do fato que se $a \subseteq \mathbb{N}$ é finito, então $\left\{x_{n}: n \in a\right\}$ é um aberto-fechado.

Exemplo 1.50. O compactificado de Čech-Stone dos naturais $\beta \mathbb{N}$ é o espaço de Stone da álgebra $\wp(\mathbb{N})$. (Isto será provado no Capítulo 5.)

Exemplo 1.51. O espaço $\omega^{*}=\beta \mathbb{N} \backslash \mathbb{N}$ é o espaço de Stone da álgebra $\wp(\mathbb{N}) /$ Fin. (Isto será provado no Capítulo d.)

Lembramos que os seguintes espaços topológicos também são espaços de Stone: o produto de Tychonoff $2^{\kappa}$ do espaço discreto $\{0,1\}, \kappa$ vezes; o compactificado de Alexandroff $\Gamma(\kappa)$ de um espaço discreto com $\kappa$ elementos; e o espaço $[0, \alpha]$ com a topologia da ordem, onde $\alpha$ é um ordinal. Porém, todos eles possuem seqüências convergentes não triviais, o que não nos interessa, uma vez que o espaço $C(K)$, onde $K$ tem seqüências convergentes não triviais não tem a propriedade de Grothendieck.

\subsubsection{A dualidade de Stone}

A dualidade de Stone vai além da representação obtida na seção anterior. Na verdade, ela tem caráter funtorial, pois traduz subespaços e imagens contínuas em imagens homomorfas e subálgebras, respectivamente. Os próximos dois resultados provam isto.

Proposição 1.52. Seja $h: A \rightarrow B$ um epimorfismo. Então, existe $f: S(B) \rightarrow S(A)$ uma função contínua e injetora, dada por $f(u)=h^{-1}[u]$. Reciprocamente, se $L \subseteq K$ são espaços booleanos, então existe $h: C l o p(K) \rightarrow C l o p(L)$ um epimorfismo, dado por $h(a)=a \cap L$.

Demonstração. Suponha $h: A \rightarrow B$ um epimorfismo. Defina $f: S(B) \rightarrow S(A)$ por $f(u)=h^{-1}[u]$. É fácil ver que $h^{-1}[u]$ é um filtro em $A$. Para provar que é ultrafiltro, 
basta notar que dado $a \in A$, ou $h(a) \in u$ ou $h(-a)=-h(a) \in u$. Logo, ou $a \in f(u)$ ou $-a \in f(u)$.

Para provar que $f$ é contínua, seja $a \in A$. Temos que

$$
\begin{aligned}
f^{-1}\left[a^{*}\right] & =\left\{u \in S(B): f(u) \in a^{*}\right\}=\left\{u \in S(B): h^{-1}[u] \in a^{*}\right\} \\
& =\left\{u \in S(B): a \in h^{-1}[u]\right\}=\{u \in S(B): h(a) \in u\} \\
& =\left\{u \in S(B): u \in h(a)^{*}\right\}=h(a)^{*},
\end{aligned}
$$

que é aberto. Logo, $f$ é contínua.

Se $u_{1} \neq u_{2} \in S(B)$, então existe $b \in B$ tal que $b \in u_{1}$ se, e somente se, $b \notin u_{2}$. Como $h$ é sobrejetor, existe $a \in A$ tal que $h(a)=b$. Logo, $a \in f\left(u_{1}\right)$ se, e somente se, $a \notin f\left(u_{2}\right)$. Portanto, $f\left(u_{1}\right) \neq f\left(u_{2}\right)$.

Reciprocamente, suponha $L \subseteq K$ espaços booleanos e considere $h: \operatorname{Clop}(K) \rightarrow$ $C l o p(L)$ dado por $h(a)=a \cap L$. É claro que $h$ está bem definido. Para mostrar que $h$ é homomorfismo, considere $a, b \in C l o p(K)$. Temos que

$$
h(a \cdot b)=(a \cap b) \cap L=(a \cap L) \cap(b \cap L)=h(a) \cdot h(b) .
$$

A demonstração das demais operações é análoga e não faremos.

Para mostrar que $h$ é sobrejetor, seja $U \subseteq L$ um aberto-fechado. Assim, existem $E, F \subseteq K$ fechados tais que $U=E \cap L$ e $L \backslash U=F \cap L$. Como $L$ é compacto, temos que $L$ é fechado e daí $U$ e $L \backslash U$ são fechados disjuntos em $K$. Como $K$ é normal e booleano, existe $a \subseteq K$ um aberto-fechado tal que $U \subseteq a$ e $L \backslash U \cap a=\emptyset$. Logo, $h(a)=U$. Portanto, $h$ é sobrejetor.

Proposição 1.53. Seja A uma álgebra de Boole e B uma subálgebra de A. Então, existe $f: S(A) \rightarrow S(B)$ uma função contínua sobrejetora, dada por $f(u)=u \cap B$. Reciprocamente, se $f: K \rightarrow L$ é uma função contínua e sobrejetora entre espaços booleanos, então existe $h: C l o p(L) \rightarrow C l o p(K)$ um monomorfismo, dado por $h(a)=f^{-1}[a]$.

Demonstração. Suponha $B$ subálgebra de $A$ e considere $f: S(A) \rightarrow S(B)$ definida por $f(u)=u \cap B$. É fácil ver que se $u \in S(A)$, então $u \cap B \in S(B)$ e, assim, $f$ está bem definida. Vejamos que $f$ é sobrejetora. Dado $u \in S(B), u$ é um filtro sobre $A$. Daí, sabemos que existe $u^{\prime} \in S(A)$ que estende $u$. Logo, $f\left(u^{\prime}\right)=u$. 
Para provar que $f$ é contínua, considere $b \in B$. Temos

$$
\begin{aligned}
f^{-1}\left[b^{*_{B}}\right] & =\left\{u \in S(A): f(u) \in b^{*_{B}}\right\}=\{u \in S(A): b \in f(u)\} \\
& =\{u \in S(A): b \in u\}=\left\{u \in S(A): u \in b^{*_{A}}\right\}=b^{*_{A}} .
\end{aligned}
$$

Logo, $f$ é contínua.

Reciprocamente, suponha $f: K \rightarrow L$ contínua e sobrejetora. Considere $h: C \operatorname{lop}(L) \rightarrow$ Clop $(K)$ definido por $h(a)=f^{-1}[a]$. É claro que $h$ está bem definido. Para mostrar que $h$ é homomorfismo, seja $a, b \in C l o p(L)$. Temos

$$
h(a \cdot b)=f^{-1}[a \cap b]=f^{-1}[a] \cap f^{-1}[b]=h(a) \cdot h(b) .
$$

As demonstrações para as demais operações são análogas e não faremos aqui.

Por fim, vejamos que $h$ é injetor: seja $a \in C \operatorname{lop}(L), a \neq 0$. Logo, existe $u \in L$ tal que $u \in a$. Como $f$ é sobrejetora, existe $v \in L$ tal que $f(v)=u$. Daí, $v \in f^{-1}[a]=h(a) \neq \emptyset$. Portanto, $h$ é injetor.

Enunciamos aqui a dualidade de Stone, para reforçar quais são as traduções que temos.

Teorema 1.54 (da dualidade de Stone). Seja $h: A \rightarrow B$ um homomorfismo de álgebras de Boole e seja $f: S(B) \rightarrow S(A)$ dada por $f(u)=h^{-1}[u]$. Então $f$ é contínua $e$

- se hé sobrejetor, então $f$ é injetora;

- se h é injetor, então f é sobrejetora.

Seja $f: L \rightarrow K$ uma função contínua e seja $h: C l o p(K) \rightarrow C l o p(L)$ dado por $h(a)=f^{-1}[a]$. Então h é um homomorfismo e

- se f é sobrejetora, então h é injetor;

- se f é injetora, então h é sobrejetor.

Demonstração. O teorema segue diretamente das duas proposições anteriores.

Notemos que o teorema acima é bastante elegante e por isso o enunciamos assim. Porém, as duas proposições anteriores fornecem intuições combinatórias mais fortes, uma vez que os homomorfismos e funções contínuas são definidos explicitamente. 


\section{ÁLGEBRAS DE BOOLE E ESPAÇOS COMPACTOS}

\subsection{Algumas traduções}

Esta última seção tem o objetivo de explicitar algumas traduções obtidas através da dualidade de Stone. São exemplos de aplicação deste resultado que serão posteriormente utilizados, ou que têm importância por si só.

\subsubsection{Cardinalidade de álgebras e propriedades dos espaços de Stone}

Proposição 1.55. Seja A uma álgebra de Boole infinita. Então, $|A|=p(S(A))$.

Demonstração. Primeiramente, note que se $B$ é uma base de abertos para $S(A)$, então para cada $a \in A$, existe $B^{\prime} \subseteq B$ tal que $a^{*}=\bigcup B^{\prime}$. Como $a^{*}$ é fechado em um espaço booleano, temos que existe $B^{\prime \prime} \subseteq B^{\prime}$ finito tal que $a^{*}=\bigcup B^{\prime \prime}$. Logo, $|A| \leq \mid\left\{B^{\prime} \subseteq B\right.$ : $B^{\prime}$ é finito $\}|=| B \mid$. Portanto, $|A| \leq p(S(A))$.

Por outro lado, $p(S(A)) \leq|A|$, uma vez que $S(A)$ tem uma base de abertos-fechados isomorfa a $A$.

Lema 1.56. Seja $\kappa$ um cardinal infinito. Todo espaço booleano de peso $\kappa$ é homeomorfo a um subespaço de $2^{\kappa}$.

Demonstração. Seja $K$ um espaço booleano de peso $\kappa$. Pela Proposição 1.55 , temos que $|C l o p(K)|=\kappa$. Considere $M=\left\{a_{\xi}: \xi<\kappa\right\} \subseteq C l o p\left(2^{\kappa}\right)$, onde $a_{\xi}=\left\{x \in 2^{\kappa}: x(\xi)=0\right\}$. É fácil ver que $\operatorname{Clop}\left(2^{\kappa}\right)=\langle M\rangle$. Seja $\phi: M \rightarrow C l o p(K)$ uma função sobrejetora. Como para qualquer $F \subseteq \kappa$ finito e toda $e \in\{-1,1\}^{F}$, temos que $\prod_{\xi \in F} e(\xi) a_{\xi} \neq \emptyset$, temos, pelo Teorema 1.22, que existe $h: C \operatorname{Cop}\left(2^{\kappa}\right) \rightarrow \operatorname{Clop}(K)$ um homomorfismo sobrejetor. Assim, pelo Teorema 1.54, $K$ é homeomorfo a um subespaço de $2^{\kappa}$.

Proposição 1.57. Seja A uma álgebra de Boole infinita. Temos que A é enumerável se, e somente se, $S(A)$ é metrizável.

Demonstração. Se $A$ é enumerável, então $p(S(A))=\omega$ e, pelo lema anterior, $S(A)$ é subespaço de $2^{\omega}$ que é metrizável. 
Reciprocamente, se $S(A)$ é metrizável, como é compacto temos que $S(A)$ tem base de abertos enumerável e daí, $|A|=p(S(A))=\omega$.

\subsubsection{FinCofin $(\mathbb{N})$ como imagem homomorfa e seqüências con- vergentes não triviais}

Proposição 1.58. Dada uma álgebra de Boole A, as seguintes afirmaçóes são equivalentes:

(i) existe uma álgebra de Boole $B$ infinita e enumerável que é imagem homomorfa de A;

(ii) $\operatorname{FinCofin}(\mathbb{N})$ é imagem homomorfa de $A$;

(iii) o espaço de Stone $K$ de A possui um subespaço infinito, fechado e metrizável.

(iv) o espaço de Stone $K$ de A possui uma seqüência convergente não trivial.

Demonstração. As implicações (i) $\Leftrightarrow$ (iii) seguem da dualidade de Stone e da proposição anterior.

As implicações (ii) $\Leftrightarrow$ (iv) seguem da dualidade de Stone e do fato que o espaço de Stone da álgebra $\operatorname{Fin} \operatorname{Cofin}(\mathbb{N})$ é uma seqüência convergente não trivial.

A implicação (iv) $\Leftrightarrow$ (iii) segue do fato que uma seqüência convergente não trivial com seu limite é um espaço infinito, fechado e metrizável.

A implicação (iii) $\Leftrightarrow$ (iv) segue do fato que todo espaço infinito, fechado e metrizável num compacto, tem uma seqüência convergente não trivial.

\subsection{3 $\wp(\mathbb{N})$ como imagem homomorfa e $\beta \mathbb{N}$ como subespaço}

Definição 1.59. Dizemos que uma família $\left(a_{\xi}\right)_{\xi<\kappa} \subseteq A$ é uma família independente se para todo $F \subseteq \kappa$ finito e todo $e \in\{-1,1\}^{F}$, tem-se que $\prod_{\xi \in F} e(\xi) a_{\xi} \neq 0$.

Proposição 1.60. Se A possui uma família independente de cardinalidade $\kappa$, então $|S(A)| \geq 2^{\kappa}$. 


\section{ÁlgEBRAS DE BOOLE E ESPAÇOS COMPACTOS}

Demonstração. Seja $X \subseteq A$ uma família independente com $|X|=\kappa$. É fácil ver que Clop $\left(2^{\kappa}\right)$ é isomorfa a $\langle X\rangle$. Como $\langle X\rangle$ é subálgebra de $A$, temos, pelo Teorema 1.54, que $2^{\kappa}$ é imagem homomorfa de $S(A)$. Do fato que $\left|2^{\kappa}\right|=2^{\kappa}$, segue que $|S(A)| \geq 2^{\kappa}$.

Proposição 1.61. Dada uma álgebra de Boole $A$, as seguintes afirmações são equivalentes:

(i) A tem uma família independente de cardinalidade $2^{\omega}$;

(ii) $\wp(\mathbb{N})$ é imagem homomorfa de $A$;

(iii) $\{0,1\}^{2^{\omega}}$ é imagem contínua do espaço de Stone $K$ de $A$.

(iv) o espaço de Stone $K$ de A possui uma cópia de $\beta \mathbb{N}$.

Demonstração. A implicação (i) $\Rightarrow($ iii), segue da demonstração da proposição anterior.

A implicação (iii) $\Rightarrow$ (i) segue da dualidade de Stone e dos fato que $C l o p\left(2^{2^{\omega}}\right)$ tem uma família independente de cardinalidade $2^{\omega}$.

As implicações (ii) $\Leftrightarrow$ (iv) seguem da dualidade de Stone e do fato que o espaço de Stone da álgebra $\wp(\mathbb{N})$ é o $\beta \mathbb{N}$.

Veremos no Capítulo 5 que $\wp(\mathbb{N})$ tem uma família independente de cardinalidade $2^{\omega}$. Daí, como imagem inversa por um homomorfismo de uma família independente é uma família independente, temos a implicação (ii) $\Rightarrow$ (i).

Para a implicação (i) $\Rightarrow\left(\right.$ ii), considere $\left(a_{\xi}\right)_{\xi<2^{\omega}} \subseteq A$ uma família independente. Tome$\operatorname{mos} \Phi:\left\{a_{\xi}: \xi<2^{\omega}\right\} \rightarrow \wp(\mathbb{N})$ bijetora. É claro que tal $\Phi$ satisfaz a condição do Primeiro Critério de Extensão de Sikorski (1.22). Assim, existe $h:\left\langle\left\{a_{\xi}: \xi<2^{\omega}\right\}\right\rangle \rightarrow \wp(\mathbb{N})$ um epimorfismo. Como $\wp(\mathbb{N})$ é completa, pelo Teorema de Extensão de Sikorski (1.28), temos que existe $\tilde{h}: A \rightarrow \wp(\mathbb{N})$ um epimorfismo que estende $h$. Assim, $\wp(\mathbb{N})$ é imagem homomorfa de $A$.

Conhecendo as Proposições 1.58 e 1.61, é interessante mencionar um famoso problema de topologia: o problema de Efimov. Sua versão topológica é: será que todo espaço topológico compacto Hausdorff infinito tem, ou uma seqüência convergente não trivial, ou uma cópia de $\beta \mathbb{N}$ ? Se analisarmos esta pergunta para o caso dos espaços booleanos, pelas proposições mencionadas, podemos traduzi-la para: será que toda álgebra de Boole 
tem ou $\operatorname{Fin} \operatorname{Cofin}(\mathbb{N})$, ou $\wp(\mathbb{N})$ como imagem homomorfa? Sabe-se que a hipótese do contínuo $(\mathrm{CH})$ implica que isto não é verdade, ou seja, que existe um espaço compacto Hausdorff infinito que não tem seqüências convergentes não triviais, nem cópias de $\beta \mathbb{N}$. Porém, ainda não se sabe se a resposta afirmativa é consistente.

\subsection{4 Álgebras completas e espaços extremamente desconexos}

Definição 1.62. Dizemos que um espaço topológico $K$ é extremamente desconexo se para todo $U \subseteq K$ aberto, $\bar{U}$ é aberto.

Não faremos aqui a demonstração, mas é possível mostrar que todo espaço compacto Hausdorff extremamente desconexo é booleano.

Proposição 1.63. Uma álgebra de Boole A é completa se, e somente se, seu espaço de Stone $S(A)$ é extremamente desconexo.

Demonstração. Seja $A$ uma álgebra completa. Seja $U \subseteq S(A)$ um aberto qualquer. Temos que existe $M \subseteq A$ tal que $U=\bigcup\left\{m^{*}: m \in M\right\}$. Como $A$ é completa, $\sum M \in A$. Note que $U$ é denso em $\left(\sum M\right)^{*}$ e este é fechado. Logo, $\left(\sum M\right)^{*}=\bar{U}$ e assim, $\bar{U}$ é aberto.

Reciprocamente, suponha que $S(A)$ é extremamente desconexo. Seja $M \subseteq A$. Note que $\overline{\bigcup\left\{m^{*}: m \in M\right\}}=\sum\left\{m^{*}: m \in M\right\} \in C l o p(S(A))$. Logo, Clop $(S(A))$ é completa e, portanto, $A$ é completa.

\subsection{5 Álgebras superatômicas e espaços dispersos}

Definição 1.64. Seja $A$ uma álgebra de Boole $A . a \in A \backslash\{0\}$ é dito um átomo, se $0<b \leq a$ implica que $b=a$. Daí, $A$ é uma álgebra de Boole atômica se para todo $a \in A \backslash\{0\}$, existe $b \leq a$ um átomo. E, por fim, $A$ é dita uma álgebra de Boole superatômica se toda imagem homomorfa $B$ de $A$ tem um átomo.

Exemplo 1.65. A álgebra $\wp(\mathbb{N})$ é atômica.

Demonstraçấo. Todo conjunto não vazio tem um átomo da forma $\{n\}$ abaixo dele. 


\section{ÁLGEBRAS DE BOOLE E ESPAÇOS COMPACTOS}

Definição 1.66. Dizemos que um espaço topológico é disperso se todo seu subespaço não vazio tem pontos isolados.

Proposição 1.67. Uma álgebra de Boole A é superatômica se, e somente se, seu espaço de Stone $S(A)$ é disperso.

Demonstração. Suponha $A$ superatômica e seja $K \subseteq S(A)$ um subespaço. Temos que $\bar{K}$ é um subespaço booleano de $S(A)$. Daí, $C l o p(\bar{K})$ é imagem homomorfa de $A$ e, portanto, existe $b \in C \operatorname{Cop}(\bar{K})$ um átomo. Seja $u \in b$. É fácil ver que $b=\{u\}$ e assim, $u$ é isolado. Se $u \in \bar{K} \backslash K, u$ não poderia ser isolado. Logo, $u \in K$ é isolado.

Reciprocamente, suponha $S(A)$ extremamente desconexo e seja $B$ uma imagem homomorfa de $A$. Temos que $S(B)$ é subespaço de $S(A)$ e, portanto, tem um ponto $u \in S(B)$ isolado. Daí, é fácil ver que existe $b \in B$ tal que $b^{*}=\{u\}$ e assim, $b$ é um átomo de $B$.

\subsubsection{Retratos de álgebras e retratos de espaços topológicos}

Definição 1.68. Seja $K$ um espaço topológico. Dizemos que um subespaço $L$ de $K$ é um retrato se existe $r: K \rightarrow L$ contínua tal que $\left.r\right|_{L}=\left.i d\right|_{L}$. Chamamos tal $r$ de retração.

Sejam $A, B$ álgebras de Boole. Dizemos que um homomorfismo $h: A \rightarrow B$ é um retrato de $A$ sobre $B$ se existe $C$ subálgebra de $A$ tal que $h_{C}: C \rightarrow B$ é um isomorfismo. Por abuso de linguagem, diremos que $B$ é retrato de $A$.

Proposição 1.69. Sejam $K, L$ espaços booleanos, $K$ subespaço de $L$. $K$ é um retrato de $L$ se, e somente se, o homomorfismo canônico $h: C l o p(L) \rightarrow C l o p(K)$ é um retrato.

Demonstração. Suponha $r: L \rightarrow K$ uma retração. Tome $C=\left\{r^{-1}[b]: b \in C l o p(K)\right\}$. $C$ é subálgebra de $C l o p(L)$, pois imagem inversa de um aberto-fechado por uma função contínua é um aberto-fechado e é fácil ver que $C$ é fechada pelas operações,$+-\mathrm{e} \cdot$. Temos assim que $\left.h\right|_{C}$ é um homomorfismo de $C$ em $C l o p(K)$.

A injetividade segue do fato que se $b \in C \operatorname{lop}(K)$ e $r^{-1}[b] \neq \emptyset$, então $b \neq 0$ e daí temos um $x \in b \subseteq K$. Logo, $x=r(x)$ e, portanto, $x \in r^{-1}[b] \cap K=h\left(r^{-1}[b]\right) \neq \emptyset$.

Vejamos que $\left.h\right|_{C}$ é sobrejetora: dado $b \in C l o p(K)$, considere $r^{-1}[b] \in C$. Vejamos que $h\left(r^{-1}[b]\right)=b$. Se $x \in b$, temos que $x=r^{-1}(x)$ e daí, $x \in r^{-1}[b]$. Por outro lado, se $x \in r^{-1}[b]$ e $x \in K$, então $x=r(x) \in b$. Logo, $h\left(r^{-1}[b]\right)=r^{-1}[b] \cap K=b$. 
Reciprocamente, suponhamos que existe $C \subseteq C \operatorname{lop}(L)$ subálgebra tal que $\left.h\right|_{C}$ é um isomorfismo de $C$ sobre $C l o p(K)$. Para cada $x \in L$, seja $u_{x}=\left\{c \in C: x \in c^{*}\right\}$. Defina $r: L \rightarrow K$ por $r(x)=h\left[u_{x}\right]$.

$r$ está bem definida, pois a intersecção de um ultrafiltro com uma subálgebra é um ultrafiltro na subálgebra e a imagem de um ultrafiltro por um homomorfismo é um ultrafiltro na imagem.

Para mostrar que $r$ é contínua, considere $b \in C l o p(K)$. Seja $c \in C$ tal que $h(c)=b$. Temos que

$$
r^{-1}\left[b^{*}\right]=\{x \in L: b \in r(x)\}=\left\{x \in L: b \in h\left[u_{x}\right]\right\}=\left\{x \in L: x \in c^{*}\right\}=c^{*},
$$

que é aberto.

Por fim, dado $y \in L$, mostremos que $h\left[u_{y}\right]=y$. Como $\left.h\right|_{C}$ é isomorfismo, temos que $h\left[u_{y}\right]=\left\{h(c): c \in u_{y}\right\}=\left\{h(c): c \in C, y \in c^{*}\right\}=\left\{b \in C l o p(K):\left(\left.h\right|_{C}\right)^{-1}(b) \in y\right\}=y$. 


\section{Capítulo 2}

\section{Espaços de Banach}

Neste capítulo, apresentamos os principais resultados da teoria de espaços de Banach que serão necessários nos próximos capítulos. O objetivo deste estudo é lembrar importantes resultados de diversos tópicos desta teoria: as topologias fraca e fraca*, algumas propriedades do operador adjunto, alguns resultados sobre subespaços complementados ${ }^{1} \mathrm{e}$, por fim, definir a propriedade de Grothendieck com alguns lemas gerais que serão úteis adiante. Analisaremos, no Capítulo 3, de que forma tais objetos e propriedades se traduzem no caso de espaços de Banach da forma $C(K)$, onde $K$ é um espaço booleano.

Nesse sentido, apresentamos primeiramente os grandes teoremas da análise. Passamos então às definições das topologias fraca e fraca* e algumas propriedades simples. Podemos destacar aqui um primeiro resultado fundamental no que segue:

Teorema (de Eberlein-Šmulian). Seja $X$ um espaço normado e $M \subseteq X$. Então, $M$ é fracamente compacto se, e somente se, $M$ é fracamente seqüencialmente compacto.

Sobre o operador adjunto, analisamos apenas algumas propriedades que precisaremos. No que se refere aos subespaços complementados, apresentamos, além da definição, alguns resultados importantes, que facilitam nossa busca por subespaços complementados nos Capítulos 5, 6 e 7. Apresentamos, por exemplo, a seguinte equivalência:

Teorema. Seja $X$ um espaço de Banach e $Y \subseteq X$ um subespaço. Então, $Y$ é comple-

\footnotetext{
${ }^{1}$ Dizemos que um subespaço $Y$ de um espaço de Banach $X$ é complementado se existe uma projeção de $X$ sobre $Y$.
} 
mentado em $X$ se, e somente se, $Y$ é fechado e existe $Z$ subespaço fechado de $X$ tal que $X=Y \oplus Z$.

Estudamos ainda alguns resultados sobre quocientes e outros do tipo da Decomposição de Pełczyński.

Por fim, na última seção, introduzimos a propriedade de Grothendieck: dizemos que um espaço de Banach $X$ tem a propriedade de Grothendieck se a convergência de seqüências nas topologias fraca e fraca* no seu dual $X^{*}$ coincidem. O objetivo desta seção é entender o que significa que um espaço de Banach possui esta propriedade, isto é, quais são as propriedades que um espaço deve possuir para que isso aconteça, ou não. Podemos notar, por exemplo, que se $X$ é um espaço reflexivo, então as topologias fraca e fraca* coincidem no espaço dual $X^{*}$ e, portanto, $X$ tem a propriedade de Grothendieck trivialmente. Convém notar ainda que os espaços estudados nos Capítulos 5, 6 e 7 têm a propriedade de Grothendieck.

Sendo o conteúdo deste capítulo bastante fundamental e clássico, deixamos diversos resultados sem demonstração e indicamos onde encontrá-los. Como referência para os tópicos aqui abordados, indicamos [Kre] para a Seção 2.1 e [Me] para as Seções 2.2, 2.3, 2.4 e 2.5. A Seção 2.6 tem resultados clássicos que também podem ser encontrados em $[\mathrm{Me}]$ e outros que estão em [Ma]. Por fim, indicamos [Gro] como referência para Seção a 2.7 .

\subsection{Preliminares}

O objetivo desta primeira seção é lembrar algumas definições e resultados importantes relativos aos espaços de Banach. Comecemos com algumas definições que serão largamente utilizadas no que segue.

Definição 2.1. Sejam $X$ e $Y$ espaços de Banach. Dizemos que uma aplicação $T: X \rightarrow Y$ é um isomorfismo de $X$ sobre $Y$, se é $T$ linear, bijetora e contínua, e $T^{-1}$ é contínua. Neste caso, dizemos que $X$ e $Y$ são isomorfos e denotamos por $X \sim Y$. Se $X, Y, Z$ são espaços de Banach tais que $X \sim Y$ e $Y$ é subespaço de $Z$, dizemos que $Y$ é uma cópia de $X$ em Z.

Mais ainda, dizemos que uma aplicação $T: X \rightarrow Y$ é uma isometria se é um 
isomorfismo tal que $\|T(x)\|=\|x\|$ para todo $x \in X$. Neste caso, dizemos que $X$ e $Y$ são isométricos e denotamos por $X \equiv Y$.

Um importante resultado que caracteriza os espaços de Banach de dimensão finita é o seguinte:

Teorema 2.2. Seja $X$ um espaço de Banach. $X$ tem dimensão finita se, e somente se, a bola unitária fechada de $X, B_{X}$ é compacta.

Demonstração. Indicamos [Kre], Teorema 2.5.3, página 77 e Teorema 2.5.5, página 80.

Definição 2.3. Seja $X$ um espaço vetorial e $M \subseteq X$ um conjunto. Definimos o subespaço gerado por $M$ (e denotamos por $[M]$ ), o seguinte espaço vetorial:

$$
[M]=\left\{\sum_{x \in M^{\prime}} \alpha_{x} x: M^{\prime} \subseteq M \text { é finito e } \alpha_{x} \in \mathbb{R}\right\} .
$$

Proposição 2.4. Seja $X$ um espaço de Banach e $Y$ um subespaço vetorial de $X$. $Y$ é fechado se, e somente se, $Y$ é Banach.

Demonstração. Indicamos [Kre], Teorema 2.3.1, página 67.

\subsection{Os grandes teoremas}

Apresentamos aqui os grandes teoremas da análise funcional, começando pelo principal deles:

Teorema 2.5 (de Hahn-Banach). Seja $X$ um espaço normado, $Y$ um subespaço de $X$ e $f: Y \rightarrow \mathbb{R}$ um funcional linear limitado. Então, existe $\tilde{f}: X \rightarrow \mathbb{R}$ um funcional linear limitado tal que $\left.\tilde{f}\right|_{Y}=f$ e $\|\tilde{f}\|=\|f\|$.

Demonstração. Indicamos [Me], Teorema 1.9.6, página 75.

Teorema 2.6 (Princípio de Limitação Uniforme). Sejam $X, Y$ espaços de Banach $e\left(T_{n}\right)_{n \in \mathbb{N}}$ uma seqüência de aplicaçôes lineares limitadas de $X$ em $Y$ tal que para cada $x \in X$, a seqüência $\left(\left\|T_{n}(x)\right\|\right)_{n \in \mathbb{N}}$ é limitada em $\mathbb{R}$. Daí, existe $M>0$ tal que $\left\|T_{n}\right\| \leq M$ para todo $n \in \mathbb{N}$. 
Demonstração. Indicamos [Me], Teorema 1.6.9, página 45.

Teorema 2.7 (da Aplicação Aberta). Sejam $X, Y$ espaços de Banach e $T: X \rightarrow Y$ uma aplicaçấo linear, limitada e sobrejetora. Então, $T$ é aberta, isto é, se $U$ é um aberto em $X$, então $T[U]$ é um aberto em $Y$. Daí, se $T$ é bijetora, então $T$ é um isomorfismo.

Demonstração. Indicamos [Me], Teorema 1.6.5, página 43.

Teorema 2.8 (do Gráfico Fechado). Sejam $X, Y$ espaços de Banach, $M \subseteq X$ e $T$ : $M \rightarrow Y$ uma aplicaçấo linear. Se o gráfico de $T$

$$
G(T)=\{(x, y) \in X \times Y: x \in M \text { e } y=T(x)\}
$$

é fechado em $X \times Y$, então $T$ é contínua.

Demonstração. Indicamos [Me], Teorema 1.6.11, página 46.

\subsection{A topologia fraca}

O objetivo desta seção é apresentar alguns resultados importantes sobre a topologia fraca, que nos serão úteis nos Capítulos $3,5,6$ e 7 .

Definição 2.9. Dado um espaço normado $X$ definimos $X^{*}$ o espaço dual de $X$ por

$$
X^{*}=\{f: X \rightarrow \mathbb{R} \mid f \text { é linear e contínua }\} .
$$

Proposição 2.10. Se $X$ é um espaço normado, então $X^{*}$ é um espaço de Banach.

Demonstração. Indicamos [Me], Teorema 1.10.7, página 87.

Definimos, finalmente, a topologia fraca:

Definição 2.11. Seja $X$ um espaço de Banach. Definimos a topologia fraca sobre $X$ como a topologia gerada por conjuntos da forma

$$
V\left(x_{0}, \phi_{1}, \ldots, \phi_{n}, \varepsilon\right)=\left\{x \in X:\left|\phi_{i}(x)-\phi_{i}\left(x_{0}\right)\right|<\varepsilon \quad \forall i, 1 \leq i \leq n\right\},
$$

onde $x_{0} \in X$, cada $\phi_{i} \in X^{*}$ e $\varepsilon>0$. Denotamos o espaço $X$ munido desta topologia por $\left(X, \sigma\left(X, X^{*}\right)\right)$. Dizemos que uma seqüência $\left(x_{n}\right)_{n \in \mathbb{N}} \subseteq X$ é fracamente convergente $a$ 


\section{ESPAÇOS DE BANACH}

$x \in X$ se converge a $x$ na topologia fraca. Por fim, dizemos que uma seqüência $\left(x_{n}\right)_{n \in \mathbb{N}} \subseteq$ $X$ é fracamente nula se é fracamente convergente a 0 .

O lema a seguir é uma caracterização bastante útil das seqüências fracamente convergentes:

Lema 2.12. Sejam $X$ um espaço de Banach, $\left(x_{n}\right)_{n \in \mathbb{N}} \subseteq X$ ex $\in X$. Então, $x_{n}$ é fracamente convergente a se, e somente se, para todo $\Phi \in X^{*}, \Phi\left(x_{n}\right)$ converge a $\Phi(x)$ em $\mathbb{R}$.

Demonstração. Suponha que $x_{n}$ converge a $x$ na topologia fraca e fixemos $\Phi \in X^{*}$. Dado $\varepsilon>0$, temos que existe $n_{0} \in \mathbb{N}$ tal que $n \geq n_{0}$ implica que $x_{n} \in V(x, \Phi, \varepsilon)$. Logo, se $n \geq n_{0}$, então $\left|\Phi\left(x_{n}\right)-\Phi(x)\right|<\varepsilon$. Portanto, $\Phi\left(x_{n}\right)$ converge a $\Phi(x)$ em $\mathbb{R}$.

Reciprocamente, suponha que para todo $\Phi \in X^{*}$, tem-se que $\Phi\left(x_{n}\right)$ converge a $\Phi(x)$ em $\mathbb{R}$. Fixemos $\Phi_{1}, \ldots, \Phi_{k} \in X^{*}$ e $\varepsilon>0$. Temos que existe, para cada $1 \leq i \leq k$, $n_{i} \in \mathbb{N}$ tal que $n \geq n_{i}$ implica que $\left|\Phi_{i}\left(x_{n}\right)-\Phi_{i}(x)\right|<\varepsilon$. Tomemos $n_{0}=\max \left\{n_{1}, \ldots, n_{k}\right\}$. Assim, se $n \geq n_{0}$, então $x_{n} \in V\left(x, \Phi_{1}, \ldots, \Phi_{k}, \varepsilon\right)$. Portanto, $x_{n}$ converge a $x$ na topologia fraca.

E ainda uma propriedade de seqüência fracamente convergente:

Lema 2.13. Sejam $X$ um espaço de Banach, $\left(x_{n}\right)_{n \in \mathbb{N}} \subseteq X$ fracamente convergente a $x \in X$. Então, $\left(x_{n}\right)_{n \in \mathbb{N}}$ é limitada.

Demonstração. Seja $\left(x_{n}\right)_{n \in \mathbb{N}} \subseteq X$ fracamente convergente a $x \in X$. Então, pelo lema acima, para toda $\Phi \in X^{*}$ temos que $\left(\Phi\left(x_{n}\right)\right)_{n \in \mathbb{N}}$ converge a $\Phi(x)$, em $\mathbb{R}$. Assim, para toda $\Phi \in X^{*}$, temos que $\left(\Phi\left(x_{n}\right)\right)_{n \in \mathbb{N}}$ é limitada em $\mathbb{R}$. Considere, para cada $\Phi \in X^{*}, r_{\Phi}>0$ tal que para todo $n \in \mathbb{N}$ temos que $\left|\Phi\left(x_{n}\right)\right| \leq r_{\Phi}$.

Consideremos ainda, para cada $n \in \mathbb{N}, c_{n} \in X^{* *}$ dado por $c_{n}(\Phi)=\Phi\left(x_{n}\right)$. Temos então que para todo $n \in \mathbb{N}$ e toda $\Phi \in X^{*},\left|c_{n}(\Phi)\right|=\left|\Phi\left(x_{n}\right)\right| \leq r_{\Phi}$. Assim, para cada $\Phi \in X^{*}$, temos que $\left(\left|c_{n}(\Phi)\right|\right)_{n \in \mathbb{N}}$ é limitada. Daí, pelo Princípio de Limitação Uniforme (2.6), existe $\alpha>0$ tal que para todo $n \in \mathbb{N},\left\|c_{n}\right\| \leq \alpha$. Como $\left\|c_{n}\right\|=\left\|x_{n}\right\|$, segue que $\left(x_{n}\right)_{n \in \mathbb{N}}$ é limitada.

Definição 2.14. Seja $M \subseteq X . M$ é dito 
- fracamente compacto se é compacto na topologia fraca;

- fracamente seqüencialmente compacto se toda seqüência $\left(x_{n}\right)_{n \in \mathbb{N}} \subseteq M$ tem subseqüência convergente na topologia fraca;

- relativamente fracamente compacto se seu fecho na topologia fraca é fracamente compacto.

Apresentamos, por fim, o principal resultado desta seção:

Teorema 2.15 (de Eberlein-Šmulian). Seja $X$ um espaço normado e $M \subseteq X$. Então, $M$ é fracamente compacto se, e somente se, $M$ é fracamente seqüencialmente compacto.

Demonstração. Indicamos [Me], Teorema 2.8.6, página 248.

\subsection{A topologia fraca*}

O objetivo desta seção é análogo ao da seção anterior: apresentar os principais resultados sobre a topologia fraca* a serem utilizados no que segue.

Definição 2.16. Sejam $X$ um espaço de Banach. Definimos a topologia fraca* sobre $X^{*}$ como a topologia gerada por conjuntos da forma

$$
V^{*}\left(\phi_{0}, x_{1}, \ldots, x_{n}, \varepsilon\right)=\left\{\phi \in X^{*}:\left|\phi\left(x_{i}\right)-\phi_{0}\left(x_{i}\right)\right|<\varepsilon \quad \forall i, 1 \leq i \leq n\right\}
$$

onde $\phi_{0} \in X^{*}$, cada $x_{i} \in X$ e $\varepsilon>0$. Denotamos o espaço $X^{*}$ munido desta topologia por $\left(X^{*}, \sigma\left(X^{*}, X\right)\right)$. Dizemos que uma seqüência $\left(\Phi_{n}\right)_{n \in \mathbb{N}} \subseteq X^{*}$ é fracamente* convergente a $\Phi$ se converge a $\Phi$ na topologia fraca*.

O lema abaixo é análogo ao Lema 2.12 e caracteriza, como o anterior, as seqüências fracamente* convergentes.

Lema 2.17. Sejam $X$ um espaço de Banach, $\left(\Phi_{n}\right)_{n \in \mathbb{N}} \subseteq X^{*} e \Phi \in X^{*}$. Então, $\Phi_{n}$ é

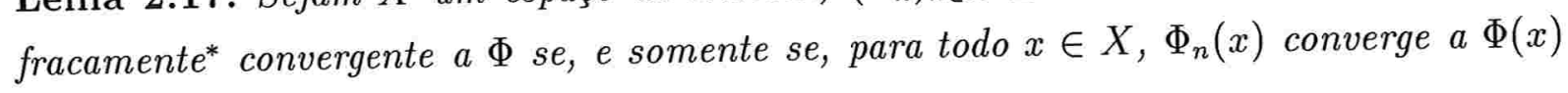
em $\mathbb{R}$. 
Demonstração. Suponha que $\Phi_{n}$ converge a $\Phi$ na topologia fraca* e fixemos $x \in X$. Dado $\varepsilon>0$, temos que existe $n_{0} \in \mathbb{N}$ tal que $n \geq n_{0}$ implica que $\Phi_{n} \in V^{*}(\Phi, x, \varepsilon)$. Logo, se $n \geq n_{0}$, então $\left|\Phi_{n}(x)-\Phi(x)\right|<\varepsilon$. Portanto, $\Phi_{n}(x)$ converge a $\Phi(x)$ em $\mathbb{R}$.

Reciprocamente, suponha que para todo $x \in X$, tem-se que $\Phi_{n}(x)$ converge a $\Phi(x)$ em $\mathbb{R}$. Fixemos $x_{1}, \ldots, x_{k} \in X$ e $\varepsilon>0$. Temos que existe, para cada $1 \leq i \leq k, n_{i} \in \mathbb{N}$ tal que $n \geq n_{i}$ implica que $\left|\Phi_{n}\left(x_{i}\right)-\Phi\left(x_{i}\right)\right|<\varepsilon$. Tomemos $n_{0}=\max \left\{n_{1}, \ldots, n_{k}\right\}$. Assim, se $n \geq n_{0}$, então $\Phi_{n} \in V^{*}\left(\Phi, x_{1}, \ldots, x_{k}, \varepsilon\right)$. Portanto, $\Phi_{n}$ converge a $\Phi$ na topologia fraca*.

Definição 2.18. Seja $M \subseteq X^{*}$. $M$ é dito fracamente* compacto se é compacto na topologia fraca*.

Por fim, um resultado clássico sobre a topologia fraca*, necessário para alguns resultados que faremos:

Teorema 2.19 (de Alaoglu). Seja $X$ um espaço normado. Se $M \subseteq X^{*}$ é limitado, então $M$ é relativamente fracamente* compacto.

Demonstração. Indicamos [Me], Teorema 2.6.18, página 229.

\subsection{O operador adjunto}

Apresentamos aqui o operador adjunto, começando por sua definição:

Definição 2.20. Sejam $X, Y$ espaços de Banach e $T: X \rightarrow Y$ linear e limitado. Definimos $T^{*}: Y^{*} \rightarrow X^{*}$, o operador adjunto, por

$$
T^{*}(f)(x)=f(T(x))
$$

Temos que $T^{*}$ é linear e limitado e $\left\|T^{*}\right\|=\|T\|$ ([Me], Proposição 3.1.2, página 284).

Temos então uma propriedade do operador adjunto bastante interessante quando estudamos isomorfismos entre espaços de Banach.

Proposição 2.21. Sejam $X, Y$ espaços de Banach e $T: X \rightarrow Y$ linear e limitado. Então: 
- (a) T é um isomorfismo sobre sua imagem se, e somente se, $T^{*}$ é sobrejetor;

- (b) T é sobrejetor se, e somente se, $T^{*}$ é um isomorfismo sobre sua imagem.

Demonstração. Indicamos [Me], Teorema 3.1.22, página 293.

A próxima proposição fornece uma propriedade do operador adjunto, bastante particular: ele leva seqüências fracamente* ${ }^{*}$ convergentes em seqüências fracamente* convergentes. Ao trabalhar com a propriedade de Grothendieck, isto pode ser muito útil:

Proposição 2.22. Sejam $X, Y$ espaços de Banach e $T: X \rightarrow Y$ linear e limitado. Então, $T^{*}: Y^{*} \rightarrow X^{*}$ é $w^{*}-w^{*}$-contínua, isto é, $T^{*}:\left(Y^{*}, \sigma\left(Y^{*}, Y\right)\right) \rightarrow\left(X^{*}, \sigma\left(X^{*}, X\right)\right)$ é contínua.

Demonstração. Considere $x_{1}, \ldots, x_{n} \in X, \Phi_{0} \in T^{*}\left[Y^{*}\right]$ e $\varepsilon>0$. Seja $\Psi_{0} \in Y^{*}$ tal que $T^{*}\left(\Psi_{0}\right)=\Phi_{0}$. Temos:

$$
\begin{aligned}
\left(T^{*}\right)^{-1}\left[V^{*}\left(\Phi_{0}, x_{1}, \ldots, x_{n}, \varepsilon\right)\right] & =\left(T^{*}\right)^{-1}\left[\left\{\Phi \in X^{*}: \forall 1 \leq i \leq n,\left|\Phi\left(x_{i}\right)-\Phi_{0}\left(x_{i}\right)\right|<\varepsilon\right\}\right] \\
& =\left\{\Psi \in Y^{*}: \forall 1 \leq i \leq n,\left|T^{*}(\Psi)\left(x_{i}\right)-T^{*}\left(\Psi_{0}\right)\left(x_{i}\right)\right|<\varepsilon\right\} \\
& =\left\{\Psi \in Y^{*}: \forall 1 \leq i \leq n,\left|\Psi\left(T\left(x_{i}\right)\right)-\Psi_{0}\left(T\left(x_{i}\right)\right)\right|<\varepsilon\right\} \\
& =V^{*}\left(\Psi_{0}, T\left(x_{1}\right), \ldots, T\left(x_{n}\right), \varepsilon\right)
\end{aligned}
$$

que é aberto em $Y^{*}$ com a topologia fraca*.

Para terminar esta seção, apresentamos a definição de aplicações fracamente compactas, a serem estudadas no contexto de espaços da forma $C(K)$. Tais aplicações têm muitas boas propriedades em espaços desta forma.

Definição 2.23. Sejam $X, Y$ espaços de Banach. Dizemos que uma aplicação linear limitada $T: X \rightarrow Y$ é fracamente compacta se leva conjuntos limitados de $X$ em conjuntos relativamente fracamente compactos de $Y$.

Proposição 2.24 (de Gantmacher). Sejam $X, Y$ espaços de Banach e $T: X \rightarrow Y$ uma aplicação linear limitada. Então, $T$ é fracamente compacta se, e somente se, $T^{*} e ́$ fracamente compacta.

Demonstração. Indicamos [Me], Teorema 3.5.13, página 343. 


\subsection{Operações em espaços de Banach}

Pretendemos apresentar algumas outras definições e resultados que serão utilizados adiante.

\subsubsection{Subespaços complementados}

Nosso objetivo aqui é lembrar resultados sobre subespaços complementados, bem como apresentar algumas propriedades interessantes.

Definição 2.25. Seja $X$ um espaço de Banach e $Y$ um subespaço de $X$. Dizemos que $P: X \rightarrow Y$ é uma projeção de $X$ sobre $Y$ se é uma aplicação linear limitada tal que $P^{2}=P$. Dizemos que $Y$ é um subespaço complementado de $X$ se existe $P: X \rightarrow Y$ uma projeção de $X$ sobre $Y$.

O primeiro exemplo de espaços complementados são os espaços de dimensão finita:

Exemplo 2.26. Se $X$ é um espaço de Banach e $Y$ é um subespaço de $X$ de dimensão ou codimensão finita, então $Y$ é complementado em $X$.

Demonstração. Indicamos [Me], Teorema 3.2.18, página 300.

Vejamos uma caracterização para os subespaços complementados em um espaço de Banach $X$. Para isto, definimos:

Definição 2.27. Se $Y$ e $Z$ são subespaços vetoriais quaisquer de um espaço vetorial $X$, então $X$ é dito a soma direta de $Y$ e $Z$ em $X$ (e escrevemos $X=Y \oplus Z$ ) se para cada $x \in X$ existem $y \in Y$ e $z \in Z$ únicos tais que $x=y+z$. Neste caso, dizemos que $Z$ é um complemento algébrico de $Y$ em $X$.

O seguinte resultado nos dá a ligação entre subespaços complementados e a existência de complementos:

Teorema 2.28. Seja $X$ um espaço de Banach e $Y \subseteq X$ um subespaço. Então, $Y$ é complementado em $X$ se, e somente se, $Y$ é fechado e existe $Z$ subespaço fechado de $X$ tal que $X=Y \oplus Z$. Neste caso, chamamos $Z$ o complemento de $Y$ em $X$. 
Demonstração. Vejamos primeiramente a volta: como para cada $x \in X$ existem $y \in Y$ e $z \in Z$ únicos tais que $x=y+z$, defina $P: X \rightarrow Y$ por $P(x)=y$. Pela unicidade, $P$ está bem definida. Tome $x_{1}, x_{2} \in X$ e $\lambda \in \mathbb{R}$. Temos $x_{1}=y_{1}+z_{1}$ e $x_{2}=y_{2}+z_{2}$, onde $y_{1}, y_{2} \in Y$ e $z_{1}, z_{2} \in Z$. Logo, $P\left(x_{1}\right)=y_{1}$ e $P\left(x_{2}\right)=y_{2}$. Daí

$$
\lambda x_{1}+x_{2}=\lambda\left(y_{1}+z_{1}\right)+\left(y_{2}+z_{2}\right)=\left(\lambda y_{1}+y_{2}\right)+\left(\lambda z_{1}+z_{2}\right),
$$

$\operatorname{com}\left(\lambda y_{1}+y_{2}\right) \in Y$ e $\left(\lambda z_{1}+z_{2}\right) \in Z$. Pela unicidade, segue que

$$
P\left(\lambda x_{1}+x_{2}\right)=\lambda y_{1}+y_{2}=\lambda P\left(x_{1}\right)+P\left(x_{2}\right) .
$$

Portanto, $P$ é linear. Além disso, $P$ é sobrejetora sobre $Y$, pois para cada $y \in Y$, temos $y=y+0$, onde $y \in Y$ e $0 \in Z$. Logo, $P(y)=y$. Daí temos também que para cada $x \in X$, sendo $y \in Y$ e $z \in Z$ tais que $x=y+z$ segue que $P^{2}(x)=P(P(x))=P(y)=y=P(x)$. Logo, $P^{2}=P$.

Para provar que $P$ limitado, considere $\left(x_{n}\right)_{n \in \omega} \subseteq X$ tal que $x_{n} \longrightarrow x \in X$ e $P\left(x_{n}\right) \longrightarrow$ $x^{\prime} \in X$. Queremos provar que $P(x)=x^{\prime}$. Como $\left(P\left(x_{n}\right)\right)_{n \in \omega} \subseteq Y$ e $Y$ é fechado, temos que $x^{\prime} \in Y$ e, portanto, $P\left(x^{\prime}\right)=x^{\prime}$. Temos também que $x_{n}-P\left(x_{n}\right) \longrightarrow x-x^{\prime}$. Mas se para cada $n, x_{n}=y_{n}+z_{n}$, onde $y_{n} \in Y$ e $z_{n} \in Z$, temos que

$$
x_{n}-P\left(x_{n}\right)=\left(y_{n}+z_{n}\right)-P\left(y_{n}+z_{n}\right)=y_{n}+z_{n}-y_{n}=z_{n}
$$

para cada $n$. Logo $\left(x_{n}-P\left(x_{n}\right)\right)_{n \in \omega} \subseteq Z$ e então, $x-x^{\prime} \in Z$, pois $Z$ é fechado. Mas para todo $z \in Z$, temos que $z=0+z$, onde $0 \in Y$ e $z \in Z$. Logo, $P(z)=0$ para todo $z \in Z$. Como $x-x^{\prime} \in Z$, segue que $P\left(x-x^{\prime}\right)=0$ e então, $P(x)=P\left(x^{\prime}\right)=x^{\prime}$. Assim, temos que o gráfico de $P$ é fechado. Daí, pelo Teorema do Gráfico Fechado (2.8), temos que $P$ é contínua e portanto, limitada.

Para a ida, note que $Y=\{x \in X: P(x)=x\}$, pois dado $y \in Y$, temos que $y=P(y)$, pela sobrejetividade de $P$ e $P^{2}=P$. Daí, como $P^{2}=P$, segue que $y=P(x)=P^{2}(x)=$ $P(P(x))=P(y)$. Por outro lado, temos que se $x \in X$ e $P(x)=x$, então $x \in P[X]=Y$. Defina $Z=P^{-1}[\{0\}]$.

Para provar que $Y$ é fechado, basta considerar $\left(y_{n}\right)_{n \in \omega} \subseteq Y$ e $y \in X$ tal que $y_{n} \rightarrow y$. Vejamos que $y \in Y$ : como cada $y_{n} \in Y$, temos que $P\left(y_{n}\right)=y_{n}$ para todo $n \in \omega$. Logo,

$$
\lim _{n \rightarrow \infty} P\left(y_{n}\right)=\lim _{n \rightarrow \infty} y_{n}=y .
$$




\section{ESPAÇOS DE BANACH}

Por outro lado, como $P$ é contínua, $P\left(y_{n}\right) \rightarrow P(y)$. Assim, $P(y)=y$ e, portanto, $y \in Y$. É claro que $Z$ é fechado, pois é imagem inversa de um fechado por uma função contínua sobrejetora.

Para todo $x \in X$, temos $x=P(x)+(x-P(x))$. Mas $P(x) \in Y$ e $P(x-P(x))=P(x)-$ $P(P(x))=P(x)-P(x)=0$. Logo, $x-P(x) \in Z$. Para mostrar a unicidade, tomemos $y_{1}, y_{2} \in Y$ e $z_{1}, z_{2} \in Z$ tais que $y_{1}+z_{1}=y_{2}+z_{2}=x$. Daí, $P\left(y_{1}+z_{1}\right)=P\left(y_{2}+z_{2}\right)=P(x)$. Mas $P\left(y_{1}+z_{1}\right)=P\left(y_{1}\right)+P\left(z_{1}\right)=y_{1}$ e $P\left(y_{2}+z_{2}\right)=P\left(y_{2}\right)+P\left(z_{2}\right)=y_{2}$. Logo, $y_{1}=y_{2}$. Mas daí, $z_{1}=x-y_{1}=x-y_{2}=z_{2}$. Logo, $P$ e $Y$ satisfazem todas as propriedades desejadas e, assim, $Y$ é complementado.

Notemos que para um espaço complementado $Y$, o complemento não é necessariamente único.

Exemplo 2.29. Considere o espaço $\mathbb{R}^{2}$ e uma reta $r \subseteq \mathbb{R}^{2}$ tal que $(0,0) \in r$. Temos que $r$ é um subespaço complementado de $\mathbb{R}^{2}$, e temos que qualquer reta $s$ tal que $(0,0) \in s$ e $s \neq r$ é um complemento para $r$.

Para estabelecermos mais uma relação entre a soma direta de espaços e um outro tipo de soma de espaços de Banach, definamos primeiramente o seguinte:

Definição 2.30. Sejam $Y, Z$ espaços de Banach. Definimos o espaço de Banach que é soma exterior de $Y$ e $Z$ (e denotamos por $Y \oplus Z$ ) por

$$
Y \oplus Z=\{(y, z) \in Y \times Z\}, \operatorname{com}\|(y, z)\|=\max \{\|y\|,\|z\|\}
$$

Não mostraremos que o espaço assim definido é, de fato, um espaço de Banach. Notemos, porém, que usamos a mesma notação $(\oplus)$ para a soma exterior e para a soma direta de espaços de Banach. O próximo resultado garante que não há confusão, uma vez que no caso em que consideramos ambas, elas coincidem:

Teorema 2.31. Seja $X$ um espaço de Banach. Se $Y$ é complementado em $X$ e $Z$ é um complemento de $Y$ em $X$, então $X$ é isomorfo a $Y \oplus Z$, onde $\oplus$ é a soma exterior.

Demonstração. Indicamos [Me], Proposição 1.8.10, página 65. 


\subsubsection{O método de decomposição de Pełczyński}

O método de decomposição de Pełczyński é um método para obter subespaços complementados isomorfos ao espaço todo. Ele nos fornece condições suficientes para isso. Vejamos:

Proposição 2.32. Suponha $X, Y$ espaços de Banach tais que $X$ é complementado em $Y$ e $Y$ é complementado em $X$. Então, $X \sim X \oplus Y$ se, e somente se, $Y \sim Y^{2}$. Daí, $X \sim X \oplus Y \sim Y$ se, e somente se $X \sim X^{2}$ e $Y \sim Y^{2}$.

Demonstraçấo. Se $X \sim X \oplus Y$, dado $Z$ tal que $Y \sim X \oplus Z$, temos

$$
Y^{2} \sim Y \oplus Y \sim X \oplus Z \oplus Y \sim X \oplus Y \oplus Z \sim X \oplus Z \sim Y
$$

Reciprocamente, se $Z$ é tal que $X=Y \oplus Z$ e $Y \sim Y^{2}$, então

$$
X \sim Y \oplus Z \sim Y^{2} \oplus Z \sim Y \oplus Y \oplus Z \sim X \oplus Y .
$$

Definição 2.33. Seja $X$ um espaço de Banach. Definimos o espaço $l_{\infty}(X)$ por

$$
l_{\infty}(X)=\left\{\left(x_{n}\right)_{n \in \mathbb{N}}: \forall n \in \mathbb{N} x_{n} \in X \quad e \sup _{n \in \mathbb{N}}\left\|x_{n}\right\|<\infty\right\}
$$

com norma $\left\|\left(x_{n}\right)_{n \in \mathbb{N}}\right\|=\sup _{n \in \mathbb{N}}\left\|x_{n}\right\|$.

Temos que o espaço assim definido é um espaço de Banach, mas não mostraremos isto aqui. Além disso, é fácil ver que para todo espaço de Banach $X, l_{\infty}(X)=\left(l_{\infty}(X)\right)^{2}$. Vejamos então uma propriedade do tipo Schroeder-Bernstein ${ }^{2}$ :

Teorema 2.34 ( de Decomposição de Pełczyński). Sejam $X, Y$ espaços de Banach tais que $X$ é complementado em $Y$ e $Y$ é complementado em $X$. Se $X \sim l_{\infty}(X)$, entâo $X \sim Y$.

Demonstração. Como $X \sim l_{\infty}(X)$, temos que $X \sim X^{2}$.

\footnotetext{
${ }^{2}$ Dada uma ordem parcial $\leq$, dizemos que ela tem a propriedade de Schroeder-Bernstein se $X \leq Y$ e $Y \leq X$ implica que $X=Y$. No nosso caso, a ordem é "ser subespaço complementado" e a igualdade é o isomorfismo.
} 


\section{ESPAÇOS DE BANACH}

Sejam $A, B$ espaços de Banach tais que $X \sim Y \oplus A$ e $Y \sim X \oplus B$. Temos

$$
\begin{aligned}
Y & \sim X \oplus A \sim X \oplus X \oplus A \sim X \oplus Y \sim l_{\infty}(X) \oplus Y \\
& \sim l_{\infty}(Y \oplus B) \oplus Y \sim l_{\infty}(Y) \oplus l_{\infty}(B) \oplus Y \\
& \sim l_{\infty}(Y) \oplus l_{\infty}(B) \sim l_{\infty}(Y \oplus B) \sim l_{\infty}(X) \sim X .
\end{aligned}
$$

\subsubsection{Quocientes}

Para aprofundar um pouco o estudo dos subespaços complementados, vejamos alguns resultados sobre espaços quociente:

Definição 2.35. Sejam $X, Y$ espaços de Banach e suponha $Y$ subespaço de $X$. Definimos a seguinte relação de equivalência em $X: x_{1} \sim x_{2}$ se $x_{1}-x_{2} \in Y$. Definimos então o espaço quociente $X / Y=\{[x]: x \in X\}$ com as operaçóes naturais e $\|[x]\|=\inf \{\| x-$ $y \|: y \in Y\}$ como norma.

Não verificaremos aqui que, de fato, este espaço está bem definido. Temos ainda os seguintes resultados, que também não serão provados:

Proposição 2.36. Se $X, Y$ são espaços de Banach e $Y$ é subespaço de $X$, então $X / Y$ é um espaço de Banach.

Demonstração. Indicamos [Me], Teorema 1.7.7, página 53.

Proposição 2.37. Sejam $X, Y$ espaços de Banach, $Y$ subespaço de $X$. Então, a aplicação $T: X \rightarrow X / Y$ dada por $T(x)=[x]$ é linear, limitada e sobrejetora.

Demonstração. Indicamos [Me], Proposição 1.7.12, página 54.

Temos então, a seguinte proposição que nos será útil mais adiante:

Proposição 2.38. Se $T: X \rightarrow Y$ é uma aplicação linear contínua sobrejetora, então $Y \sim X / \operatorname{Ker}(T)$. 
Demonstração. Considere $i: X / K \operatorname{er}(T) \rightarrow Y$ dada por $i([x])=T(x)$. $i$ está bem definida, pois se $\left[x_{1}\right]=\left[x_{2}\right]$, então $x_{1}-x_{2} \in \operatorname{Ker}(T)$ e daí, $T(x)=T(y)$. A linearidade de $i$ segue da linearidade de $T$.

$i$ é injetora, pois se $i([x])=0$, então $T(x)=0$ e daí $x \in \operatorname{Ker}(T)$. Logo, $[x]=0$. A sobrejetividade de $i$ segue da sobrejetividade de $T$.

Por fim, fixemos $x \in X$. Dado $\varepsilon>0$, temos que existe $z \in \operatorname{Ker}(T)$ tal que $\|x-z\| \leq$ $\|[x]\|+\varepsilon$, pela definição de $\|[x]\|$. Daí, temos

$$
\|i([x])\|=\|T(x)\|=\|T(x-z)\| \leq\|T\| \cdot\|x-z\| \leq\|T\| \cdot(\|x\|+\varepsilon) .
$$

Logo, $\|i([x])\| \leq\|T\| \cdot\|[x]\|$. Portanto, $T$ é contínua e, pelo Teorema da Aplicação Aberta (2.7), temos que $T$ é um isomorfismo.

Corolário 2.39. Sejam $X, Y$ espaços de Banach tais que $Y$ é complementado em $X$. Então $Y$ é isomorfo a um quociente de $X$.

Demonstração. Se $Y$ é complementado em $X$ então existe $P: X \rightarrow Y$ uma projeção. Segue, da proposição anterior, que $Y \sim X / \operatorname{Ker}(P)$.

\subsection{A propriedade de Grothendieck}

Queremos, nesta seção, introduzir a propriedade de Grothendieck, que será explorada nos Capítulos 3, 5, 6 e 7. Vejamos primeiramente sua definição:

Definição 2.40. Dizemos que um espaço de Banach $X$ tem a propriedade de Grothendieck, ou $X$ é um espaço de Grothendieck, se as convergências fraca e fraca* de seqüências coincidem no espaço $X^{*}$, dual de $X$.

Podemos fazer algumas observações para tentar entender um pouco melhor esta propriedade. Em primeiro lugar, dado um espaço de Banach $X$, como a topologia fraca em $X^{*}$ é induzida por elementos de $X^{* *}$ e a topologia fraca* é induzida por elementos de $X$, e ainda, $X$ está imerso em $X^{* *}$, temos que a convergência fraca sempre implica na convergência fraca*. Assim, precisamos verificar apenas a outra implicação. 


\section{ESPAÇOS DE BANACH}

Se consideramos $X$ um espaço reflexivo, temos que as duas topologias coincidem e, portanto, a convergência em cada uma delas também coincide. Assim, o caso $X$ reflexivo é trivial para esta propriedade e assim, não o consideraremos.

Vejamos um primeiro exemplo de espaço que não possui esta propriedade:

Exemplo 2.41. $c_{0}$ não tem a propriedade de Grothendieck.

Demonstração. Considere $\left(f_{n}\right)_{n \in \mathbb{N}} \subseteq c_{0}^{*}$ a seqüência de funcionais tal que se $x=\left(\xi_{n}\right)_{n \in \mathbb{N}} \in$ $c_{0}$, então $f_{n}(x)=\xi_{n}$. É fácil ver que, de fato, $f_{n} \in c_{0}^{*}$. Assim, temos que para todo $x \in c_{0}$, $f_{n}(x)=\xi_{n} \rightarrow 0$ quando $n \rightarrow \infty$. Logo, pelo Lema $2.12,\left(f_{n}\right)_{n \in \mathbb{N}}$ é fracamente ${ }^{*}$ convergente a 0 .

Por outro lado, considere $\Phi \in c_{0}^{* *}$, dado por $\Phi(f)=\sum_{n \in \mathbb{N}} f\left(e_{2 n}\right)$. É fácil ver que, de fato, $\Phi \in c_{0}^{* *}$. Temos que $\Phi\left(f_{n}\right)=1$, se $n$ é par e $\Phi\left(f_{n}\right)=0$ se $n$ é ímpar Portanto, $\left(f_{n}\right)_{n \in \mathbb{N}}$ não converge fracamente a 0 e assim, $c_{0}$ não é Grothendieck.

Outro exemplo de espaços com a propriedade de Grothendieck são os quocientes de espaços com a propriedade de Grothendieck. Vejamos:

Proposição 2.42. Sejam $X, Y$ espaços de Banach e $T: X \rightarrow Y$ linear, limitada $e$ sobrejetora. Se $X$ tem a propriedade de Grothendieck, então $Y$ tem a propriedade de Grothendieck.

Demonstração. Seja $\left(f_{n}\right)_{n \in \mathbb{N}} \subseteq Y^{*}$ e $f \in Y^{*}$ tais que $f_{n}$ converge na topologia fraca* a f. Daí, $\left(T^{*}\left(f_{n}\right)\right)_{n \in \mathbb{N}} \subseteq X^{*}, T^{*}(f) \in X^{*}$. Como $T^{*}$ é $w^{*}$ - $w^{*}$-contínua ${ }^{3}, T^{*}\left(f_{n}\right)$ converge na topologia fraca* a $T^{*}(f)$. Como $X$ é Grothendieck, $T^{*}\left(f_{n}\right)$ converge fracamente a $T^{*}(f)$.

Sejam $\phi_{1}, \ldots, \phi_{m} \in Y^{* *}$ e $\varepsilon>0$. Como $T$ é sobrejetora, temos que $T^{* *}$ é sobrejetora pela Proposição 2.21. Logo, existem $\psi_{1}, \ldots, \psi_{m} \in X^{* *}$ tais que $T^{* *}\left(\psi_{i}\right)=\phi_{i}$ para todo $1 \leq i \leq m$. Daí, existe $n_{0} \in \mathbb{N}$ tal que se $n \geq n_{0}$, então $\left|\psi_{i}\left(T^{*}\left(f_{n}\right)\right)-\psi_{i}\left(T^{*}(f)\right)\right|<\varepsilon$ para todo $1 \leq i \leq m$.

Como

$$
\psi_{i}\left(T^{*}\left(f_{n}\right)\right)-\psi_{i}\left(T^{*}(f)\right)=\psi_{i}\left(T^{*}\left(f_{n}-f\right)\right)=T^{* *}\left(\psi_{i}\right)\left(f_{n}-f\right)=\phi_{i}\left(f_{n}-f\right),
$$

\footnotetext{
${ }^{3} \mathrm{Se} X, Y$ são espaços de Banach, dizemos que uma aplicação linear $T: X^{*} \rightarrow Y^{*}$ é $w^{*}$-w*-contínua se é contínua com relação às topologias fraca* de $X^{*}$ e $Y^{*}$.
} 
segue que para todo $1 \leq i \leq m,\left|\phi_{i}\left(f_{n}\right)-\phi_{i}(f)\right|<\varepsilon$. Portanto, $f_{n}$ converge a $f$ na topologia fraca.

Corolário 2.43. Sejam X,Y espaços de Banach, $Y$ subespaço de $X$. Se $X$ é de Grothendieck, então $X / Y$ é de Grothendieck.

Demonstração. Pela Proposição 2.37, temos que $T: X \rightarrow X / Y$ dada por $T(x)=[x]$ é linear, limitada e sobrejetora. Assim, pela proposição anterior, se $X$ é Grothendieck, então $X / Y$ é Grothendieck.

Temos assim um pequeno painel de como se comporta a propriedade de Grothendieck e obtemos um pouco de intuição neste sentido, para trabalharmos com ela no contexto de espaços $C(K)$. 


\section{Capítulo 3}

\section{Espaços de Banach da forma $C(K)$}

Neste capítulo fazemos o estudo dos espaços de Banach de funções contínuas, incluindo os principais resultados a serem utilizados nos Capítulos 5,6 e 7. Pretendemos analisar, paralelamente ao capítulo anterior, o que o fato de ser um espaço de funções contínuas acrescenta à estrutura de um espaço de Banach. Com esse intuito, analisaremos como se comportam as topologias fraca e fraca*, os subespaços e subespaços complementados, os operadores fracamente compactos e a propriedade de Grothendieck, cujos exemplos serão apresentados nos Capítulos 5, 6 e 7.

Para analisar a estrutura de tais espaços, começamos apresentando alguns clássicos teoremas que permitem o intercâmbio entre a estrutura de espaço de Banach e a estrutura de espaço de funções contínuas: o Teorema de Stone-Weierstrass e o Teorema de Representação de Riesz. Este último será utilizado em todos os capítulos que seguem:

Teorema de Representação de Riesz. Se K é um espaço topológico compacto Hausdorff, então para todo funcional linear limitado $\xi$ sobre $C(K)$ existe uma única medida de Radon $\mu$ sobre $K$ tal que

$$
\xi(f)=\int_{K} f d \mu
$$

para toda $f \in C(K)$. Neste caso, $\|\xi\|=\|\mu\|$.

Utilizando tais resultados, obtemos boas propriedades das topologias fraca e fraca* em espaços da forma $C(K)$. Obtemos condições necessárias para seqüências serem convergentes nestas topologias. Tais resultados encontram-se nas Seções 3.2, 3.3 e 3.4. 
No contexto de subespaços e subespaços complementados, estudamos alguns exemplos de subespaços complementados em espaços $C(K)$. Mostramos, por exemplo, que dados $L \subseteq K$ espaços booleanos, se $L$ é retrato de $K$, então $C(L)$ é complementado em $C(K)$.

Passamos então a questões relacionadas aos espaços $c_{0}$ e $l_{\infty}$ e pretendemos estudar os superespaços de $c_{0}$ e $l_{\infty}$. Apresentamos resultados que respondem perguntas do tipo: "quais são seus superespaços?" ou "quando é que $c_{0}$ e $l_{\infty}$ são complementados?". Sobre o $c_{0}$, podemos destacar o seguinte resultado:

Teorema (de Sobczyk). Se X é um espaço de Banach separável e co é subespaço de $X$, então c có complementado em $X$.

Este teorema, combinado com outro resultado, garante que todo espaço da forma $C(K)$ separável tem uma cópia complementada de $c_{0}$. Veremos adiante que isto implica que tais $C(K)$ não são espaços de Grothendieck.

No que se refere ao $l_{\infty}$, temos sua injetividade ${ }^{1}$ como principal resultado:

Teorema. Se X é um espaço de Banach e $l_{\infty}$ é subespaço de $X$, então $l_{\infty}$ é complementado em $X$.

Salientamos que diversos dos resultados sobre espaços da forma $C(K)$ valem para qualquer espaço compacto Hausdorff $K$. Faremos, porém, a demonstração apenas para espaços booleanos, sendo a demonstração neste caso mais elegante. Optamos por isso, pois os exemplos dos Capítulos 5, 6 e 7 são espaços $C(K)$, onde $K$ é booleano. Além disso, notemos que se $K$ é um compacto Hausdorff métrico, então $C(K)$ é isomorfo a $C(L)$, para algum espaço booleano $L$ (veja [Ros2]). Para o caso geral, os primeiros exemplos de espaços compactos Hausdorff $K$ tais que $C(K)$ não é isomorfo a $C(L)$ para nenhum $L$ booleano foram obtidos apenas recentemente (veja [Kosz]). Entendemos assim que os espaços da forma $C(K)$, onde $K$ é booleano têm um papel bastante significativo na teoria de espaços de Banach da forma $C(K)$, onde $K$ é um compacto Hausdorff qualquer.

A Seção 3.6 tem por objetivo a apresentação de dois resultados combinatórios, cuja aplicação neste trabalho é para os espaços de Banach da forma $C(K)$. O principal deles

\footnotetext{
${ }^{1}$ Dizemos que um espaço de Banach $X$ é injetivo se, para quaisquer espaços de Banach $Y, Z$ tais que $Y$ é subespaço de $Z$ e para qualquer aplicação linear limitada $T: Y \rightarrow X$, existe $\tilde{T}: Z \rightarrow X$ linear e limitada que estende $T$. O teorema enunciado equivale a esta propriedade para o espaço $l_{\infty}$.
} 
é chamado Lema de Rosenthal.

Como os operadores fracamente compactos foram muito utilizados no entendimento de certos espaços da forma $C(K)$, como o $l_{\infty}$ e o $l_{\infty} / c_{0}$, este tópico é o assunto da Seção 3.7. O principal resultado fornece a equivalência entre aplicações fracamente compactas e outras três propriedades:

Proposição. Sejam $K, L$ espaços booleanos e $T: C(K) \rightarrow C(L)$ uma aplicação linear limitada. São equivalentes:

(a) T é fracamente compacta;

(b) $T$ é completamente contínua ${ }^{2}$;

(c) não existe $X$ uma cópia de $c_{0}$ em $C(K)$ tal que $\left.T\right|_{X}$ é um isomorfismo sobre sua imagem;

(d) para toda anticadeia $\left(a_{n}\right)_{n \in \mathbb{N}} \subseteq C l o p(K)$ tem-se que $\left(T\left(\chi_{a_{n}}\right)\right)_{n \in \mathbb{N}}$ converge a 0 (na norma).

Com esta caracterização, os principais resultados para $C(K)$ usando operadores fracamente compactos podem ser obtidos usando linguagem combinatória.

Por fim, analisamos as propriedades de espaços de Grothendieck da forma $C(K)$. Vimos anteriormente que espaços reflexivos têm, trivialmente, a propriedade de Grothendieck. Assim, temos um primeiro exemplo de espaço $C(K)$ Grothendieck: o espaço $\mathbb{R}^{n}$, para todo $n \in \mathbb{N}$, é de Grothendieck e $\mathbb{R}^{n}=C(K)$, onde $|K|=n$ e $K$ tem a topologia discreta. Sendo este caso trivial, consideraremos apenas espaços $C(K)$ de dimensão infinita e, portanto, $K$ infinito.

Pretendemos obter aqui uma idéia geral de como se comportam tais espaços, para posteriormente considerarmos casos específicos e analisá-los. Podemos destacar, dentre suas propriedades, o seguinte:

Teorema. Seja K um espaço booleano. São equivalentes as seguintes afirmações:

\footnotetext{
${ }^{2}$ Dizemos que uma aplicação linear $T: X \rightarrow Y$, onde $X$ e $Y$ são espaços de Banach, é completamente contínua se leva seqüências de $X$ que convergem fracamente a 0 em seqüências de $Y$ convergentes a 0 na norma.
} 
(a) $C(K)$ é de Grothendieck;

(b) $C(K)$ não possui cópias complementadas de $c_{0}$.

Este resultado caracteriza os espaços de Grothendieck da forma $C(K)$, para $K$ booleano, através de seus subespaços complementados. Podemos, assim, considerar a propriedade de Grothendieck em espaços $C(K)$ como uma tradução para o fato que $K$ não tem seqüências convergentes não triviais. Notemos, porém, que não devemos esperar uma tradução fiel entre $K$ e $C(K)$, como aquela que temos entre álgebras de Boole e espaços booleanos, uma vez que existem espaços compactos Hausdorff não homeomorfos $K$ e $L$ tais que $C(K)$ é isomorfo a $C(L)$. Assim, esta tradução do fato que $K$ não tem seqüências convergentes não triviais para $C(K)$ ter a propriedade de Grothendieck não é perfeita: existem espaços da forma $C(K)$ sem a propriedade de Grothendieck, onde $K$ não tem seqüências convergentes não triviais (veja Exemplo 4.10 de [Scha]). Cada um dos Capítulos 5, 6 e 7 possui exemplos de espaços de Grothendieck $C(K)$.

Como referência para os tópicos aqui abordados, indicamos [Se] para as Seções 3.1 e 3.2. e [Di] para as Seções 3.3 e 3.4. A referência [Ma] faz a maioria dos resultados da Seção 3.5 em detalhes. Indicamos [Ros1] para os resultados combinatórios da Seção 3.6 e [DU] como referência para a Seção 3.7. Porém, a linguagem lá utilizada é a de medidas vetoriais e a que optamos por utilizar aqui é bastante combinatória. Assim, indicamos também [Di] para esta mesma seção. Por fim, indicamos [Scha] para a Seção 3.8, uma vez que faz-se lá um estudo aprofundado sobre a relação da propriedade de Grothendieck com outras propriedades clássicas ${ }^{3}$.

\subsection{Resultados úteis}

Começamos o capítulo apresentando alguns resultados sobre espaços de funções contínuas que permitirão obter interessantes propriedades sobre estrutura de tais espaços como espaços de Banach.

\footnotetext{
${ }^{3}$ A propriedade de Nikodym, a propriedade de Vitali-Hahn-Sacks, etc.
} 


\subsubsection{O Teorema de Stone-Weierstrass}

Comecemos com uma definição:

Definição 3.1. Dizemos que $M \subseteq C(K)$ separa pontos de $K$ se para todos $x, y \in K$ com $x \neq y$, existe $f \in M$ tal que $f(x) \neq f(y)$.

O primeiro resultado que apresentamos fornece um conjunto denso em $C(K)$, onde $K$ é booleano, com propriedades tais que permitem argumentos combinatórios: o conjunto das funções simples sobre conjuntos abertos-fechados de $K$.

Teorema 3.2 (de Stone-Weierstrass). Seja $K$ um espaço compacto Hausdorff. Suponhamos $W \subseteq C(K)$ com as seguintes propriedades:

- dados $f, g \in W$, então $f+g, f \cdot g \in W$;

- W contém as funções constantes;

- W separa pontos de $K$.

Então $\bar{W}=C(K)$.

Demonstração. Indicamos [Eng], Teorema 3.2.21, página 144.

Definição 3.3. Seja A uma subálgebra de Boole de $\wp(X)$ para um conjunto $X$. Dizemos que $f$ é uma função simples sobre $A$ se $f=\sum_{k=1}^{n} r_{k} \chi_{a_{k}}$, onde $a_{1}, \ldots, a_{n} \in A$ e $a_{1} \cup \ldots \cup$ $a_{n}=1$ e $r_{1}, \ldots, r_{n} \in \mathbb{R}$. Denotamos por $\mathcal{W}_{0}(A)$ o conjunto das funçốes simples sobre $A$.

Notemos que se $K$ é booleano, $\mathcal{W}_{0}(C l o p(K))$ possui as três propriedades da hipótese do resultado acima. Assim, se $K$ é booleano, temos que $\overline{\mathcal{W}_{0}(C l o p(K))}=C(K)$. Vejamos que, neste caso, a densidade de $C(K)$ é a cardinalidade da álgebra $C l o p(K)$.

Proposição 3.4. Seja $K$ um espaço booleano. Então, $p(K)=d(C(K))$.

Demonstração. Suponha que $K$ tem peso $\kappa$. Pela Proposição 1.55 , temos que $|C l o p(K)|=$ $\kappa$. Sabemos ainda, pelo Teorema de Stone-Weierstrass (3.2), que o conjunto

$$
D=\left\{\sum_{i=1}^{n} r_{i} \chi_{a_{i}}: n \in \mathbb{N}, r_{i} \in \mathbb{Q}, a_{i} \in C l o p(K)\right\}
$$


é denso em $C(K)$. Mas $|D| \leq\left|[C l o p(K)]^{<\omega}\right| \cdot\left|[\mathbb{Q}]^{<\omega}\right| \cdot|\mathbb{N}|=|C \operatorname{lop}(K)| \cdot \omega \cdot \omega=\kappa$. Logo, $d(C(K)) \leq \kappa$.

Suponhamos agora que $d(C(K))=\kappa$. Seja então $D \subseteq C(K)$ denso com $|D|=\kappa$. Daí,

$$
B=\left\{f^{-1}[(p, q)]: f \in D, p, q \in \mathbb{Q}, p<q\right\}
$$

forma uma base de abertos para $K$ e $|B| \leq|D| \cdot\left|[\mathbb{Q}]^{2}\right|=|D| \cdot \omega=|D|=\kappa$. Portanto, $p(K) \leq d(C(K))$ e temos o resultado.

\subsubsection{O Teorema de Representação de Riesz}

Vejamos agora o Teorema de Representação de Riesz. Este resultado nos fornece uma representação para funcionais sobre $C(K)$ como medidas sobre $K$. Esta representação é bastante interessante, uma vez que podemos ter uma intuição geométrica de tais objetos. Para entendê-lo, precisamos ainda de algumas definições.

Definição 3.5. $\mu$ é dita uma medida finitamente aditiva sobre uma álgebra de Boole $A$, ou simplesmente uma medida sobre $A$, se é uma função $\mu: A \rightarrow \mathbb{R}$ tal que $\mu(0)=0$ e para todos $a_{1}, \ldots, a_{n} \in A$ dois a dois disjuntos,

$$
\mu\left(\sum_{i=1}^{n} a_{i}\right)=\sum_{i=1}^{n} \mu\left(a_{n}\right) .
$$

Denotaremos por $\mathcal{N}(A)$ o conjunto de todas as medidas sobre $A$. Diremos que $\mu$ é uma medida sobre um espaço topológico $K$, se $\mu$ é uma medida sobre uma subálgebra $A$ de $\wp(K)$.

Definição 3.6. Seja $\mu$ uma medida sobre A. Se $f \in \mathcal{W}_{0}(A), f=\sum_{k=1}^{n} r_{k} \chi_{a_{k}}$, onde $a_{1} \cup \ldots \cup a_{n}=1$ e $r_{1}, \ldots r_{n} \in \mathbb{R}$, então a integral de $f$ com respeito a $\mu$ é definida por

$$
\xi_{\mu}(f)=\int f d \mu=\sum_{k=1}^{n} r_{k} \mu\left(a_{k}\right) .
$$

Se $a \in A$, então $\int_{a} f d \mu=\xi_{\mu}\left(f \chi_{a}\right)$.

Definição 3.7. Dizemos que uma medida $\mu$ sobre A é uma medida limitada se

$$
\sup \{|\mu(a)|: a \in A\}<\infty .
$$


Neste caso, definimos a variação de $\mu$ sobre um $a \in A$ por

$$
|\mu|(a)=\sup \left\{\sum_{i=1}^{n}\left|\mu\left(a_{i}\right)\right|: a=\bigcup_{i=1}^{n} a_{i} \text { e } a_{i} \cap a_{j}=0 \text { se } i \neq j\right\} .
$$

Denotaremos por $\mathcal{N}_{b}(A)$ o conjunto das medidas sobre A limitadas. Definimos a norma de uma medida limitada $\mu$ por $\|\mu\|=|\mu|(1)$. Notemos que se $\mu$ é uma medida limitada sobre um espaço topológico $K$, então $\|\mu\|=|\mu|(K)$ e se, mais ainda, $\mu$ é não negativa, entẫo $|\mu|(a)=\mu(a)$ para todo $a \in A$ e $\|\mu\|=\mu(K)$.

Definição 3.8. Se $\mu$ é uma medida limitada sobre $A$, então o funcional $\xi_{\mu}$ sobre $\mathcal{W}_{0}(A)$ tem uma única extensão a $\mathcal{W}(A)$, o completamento de $\mathcal{W}_{0}(A)$. Se $f \in \mathcal{W}(A)$, então o valor desta extensão em $f$ será denotado por $\int f d \mu$ e será chamado a integral de $f$ com respeito a $\mu$. Se $a \in A$, então $\int_{a} f d \mu$ é definido como $\int f \cdot \chi_{a} d \mu$.

Definição 3.9. Uma medida boreliana sobre um espaço topológico $K$ é uma medida $\sigma$-aditiva sobre a álgebra dos conjuntos borelianos ${ }^{4} \operatorname{Bor}(K)$, isto é, uma medida $\mu$ sobre $\operatorname{Bor}(K)$ tal que se $\left(B_{n}\right)_{n \in \mathbb{N}} \subseteq$ Bor $(K)$ é uma família de borelianos dois a dois disjuntos, então

$$
\mu\left(\sum_{n \in \mathbb{N}} B_{n}\right)=\sum_{n \in \mathbb{N}} \mu\left(B_{n}\right) .
$$

Sabemos que para conjuntos borelianos $\sum_{n \in \mathbb{N}} B_{n}=\bigcup_{n \in \mathbb{N}} B_{n} \in \operatorname{Bor}(K)$, pois Bor $(K)$ é uma álgebra $\sigma$-completa.

Definição 3.10. Um medida boreliana limitada $\mu$ é uma medida regular se para todo $B \in \operatorname{Bor}(K)$ e todo $\varepsilon>0$ existem conjuntos $G$ aberto e $F$ fechado tais que $F \subseteq B \subseteq G$ e $|\mu|(G \backslash F)<\varepsilon$.

Definição 3.11. Uma medida $\mu$ sobre $K$ é uma medida de Radon se é uma medida boreliana limitada regular. Denotamos por $\mathcal{M}(K)$ o conjunto de todas as medidas de Radon sobre $K$.

Apresentamos aqui um resultado que será freqüentemente utilizado:

Lema 3.12. Seja $K$ um espaço compacto Hausdorff e $\mu$ uma medida de Radon sobre $K$. Então, $|\mu|$ é uma medida de Radon não negativa sobre $K$.

\footnotetext{
${ }^{4} \mathrm{~A}$ álgebra dos conjuntos borelianos de $K$ é a menor álgebra de Boole $\sigma$-completa de $\wp(K)$ que contém os subconjuntos abertos de $K$.
} 
Demonstração. Indicamos [Se], Teorema 17.2.2, página 289.

Vejamos finalmente o Teorema de Representação de Riesz:

Teorema 3.13 (de Representação de Riesz). Se K é um espaço compacto Hausdorff, então para todo funcional linear limitado $\xi$ sobre $C(K)$ existe uma única medida de Radon $\mu$ sobre $K$ tal que

$$
\xi(f)=\int_{K} f d \mu
$$

para toda $f \in C(K)$. Neste caso, $\|\xi\|=\|\mu\|$.

Demonstração. Indicamos [Se], Teorema 8.4.1, página 312.

Notemos que, da forma como definimos, temos que medidas de Radon são casos particulares de medidas finitamente aditivas sobre uma álgebra de Boole. Porém, na realidade, medidas finitamente aditivas sobre álgebras de Boole podem ser interpretadas como medidas de Radon sobre seu espaço de Stone. Vejamos de que forma isto ocorre.

Temos que, pelo teorema anterior, um funcional linear e limitado $\xi$ sobre $C(K)$, isto é, um elemento $\xi$ de $C(K)^{*}$, pode ser representado como a integral com respeito a uma medida de Radon $\mu$ sobre $K$, ou seja, uma medida regular $\mu, \sigma$-aditiva sobre $\operatorname{Bor}(K)$. Mas vimos que o Teorema de Stone-Weierstrass (3.2) garante que para espaços booleanos $K$, $C(K)=\overline{\mathcal{W}_{0}(C l o p(K))}$. Daí, $\xi$ está bem determinada por seu valor nas funções simples sobre $C l o p(K)$. Assim, $\xi$ pode ser representado como a integral com respeito a uma medida finitamente aditiva $\nu$ sobre $C l o p(K)$, onde $\nu=\left.\mu\right|_{C l o p(K)}$.

Porém, temos que $\mu$ é $\sigma$-aditiva e $\nu$ é apenas finitamente aditiva, pois, para começar, temos que $C l o p(K)$ é uma subálgebra de $\operatorname{Bor}(K)$ e $\operatorname{Bor}(K)$ é $\sigma$-completa, mas $C l o p(K)$ não o é. Além disso, se $M=\left\{m_{n}: n \in \mathbb{N}\right\} \subseteq C l o p(K)$ é uma anticadeia, mesmo que exista $\sum_{C l o p(K)} M \in C l o p(K)$, podemos ter que $\sum_{C l o p(K)} M \neq \sum_{B o r(K)} M=\bigcup M$. Suponha que para algum tal $M$, temos que $\sum_{C l o p(K)} M=\sum_{B o r(K)} M=\bigcup M$. Neste caso, $\bigcup M \in C l o p(K)$ e daí, $\bigcup M$ é um fechado em um compacto Hausdorff e, portanto, é compacto. Como $M$ é um recobrimento de $\bigcup M$ por abertos, temos que existe $n \in \mathbb{N}$ tal que $\bigcup M=\bigcup_{i=0}^{n} m_{n}$ e daí, $m_{i}=0$ para $i>n$. Assim, na realidade, não existem uniões infinitas em $C l o p(K)$ e, portanto, toda medida finitamente aditiva sobre $C l o p(K)$ é $\sigma$-aditiva com respeito a uniões, por vacuidade. 
Não faremos a demonstração aqui, mas temos que, reciprocamente, se $\nu$ é uma medida finitamente aditiva sobre $C l o p(K)$, então $\nu$ pode ser estendida a uma única medida limitada $\mu \sigma$-aditiva regular sobre álgebra $\sigma$-completa gerada por $C l o p(K)$ que é $\operatorname{Bor}(K)$, isto é, uma única medida de Radon $\mu$ sobre $K$.

Conseqüentemente, temos uma correspondência biunívoca entre as medidas finitamente aditivas sobre $C l o p(K)$ e as medidas de Radon sobre $K$. Pode-se provar ainda que $\nu$ será $\sigma$-aditiva sobre $C l o p(K)$ (com respeito à operação $\sum$ ) se, e somente se, para toda $\left(a_{n}\right)_{n \in \mathbb{N}} \subseteq C \operatorname{lop}(K)$, tal que o fecho de $a$ é aberto, onde $a=\bigcup_{n \in \mathbb{N}} a_{n}$, tem-se que $\nu$ na fronteira de $a$ é zero. Um exemplo de uma tal medida é quando seu valor é zero em todo subconjunto raro de $K$. Indicamos [Se] para mais detalhes.

Vamos identificar, a partir de agora, cada seqüência de elementos de $C(K)^{*}$ com a seqüência de medidas de Radon dada pelo Teorema de Representação de Riesz.

Ainda mais um resultado sobre espaços de funções, que, combinado com o Teorema de Representação de Riesz, é bastante útil quando entendemos as topologias fraca e fraca* em tais espaços:

Teorema 3.14 (da Convergência Dominada de Lebesgue). Seja $K$ um espaço compacto Hausdorff. Suponhamos que $\left(f_{n}\right)_{n \in \mathbb{N}} \subseteq C(K)$ converge pontualmente a $f \in C(K)$ e existem $\mu \in C(K)^{*}$ e $g: K \rightarrow \mathbb{R}$ tais que $\int_{K} g d \mu<\infty$. Se para todo $n \in \mathbb{N}$, tem-se que $\left|f_{n}\right| \leq g$, então

$$
\lim _{n \rightarrow \infty} \int_{K} f_{n} d \mu=\int_{K} f d \mu
$$

Demonstração. Indicamos [Se], Teorema 19.2.6, página 329.

\subsection{A topologia fraca em $C(K)$}

Vejamos como se comporta a topologia fraca em espaços da forma $C(K)$ :

Proposição 3.15. Seja $K$ um espaço compacto Hausdorff. Temos que $\left(f_{n}\right)_{n \in \mathbb{N}} \subseteq C(K)$ converge fracamente a $f \in C(K)$ se, e somente se, $\left(f_{n}\right)_{n \in \mathbb{N}}$ converge pontualmente a $f$ e é limitada. 
Demonstração. Suponha que $\left(f_{n}\right)_{n \in \mathbb{N}} \subseteq C(K)$ converge fracamente a $f \in C(K)$. Segue, do Lema 2.13, que $\left(f_{n}\right)_{n \in \mathbb{N}}$ é limitada.

Além disso, dado $x \in K$, definimos $\Phi_{x}: C(K) \rightarrow \mathbb{R}$ por $\Phi_{x}(g)=g(x)$. $\Phi_{x}$ está bem definida e é fácil ver que $\Phi_{x}$ é linear. $\Phi_{x}$ é limitada, pois dada $g \in C(K)$, temos

$$
\left\|\Phi_{x}(g)\right\|=|g(x)| \leq\|g\| .
$$

Assim $\Phi_{x} \in C(K)^{*}$ e, como $\left(f_{n}\right)_{n \in \mathbb{N}}$ converge fracamente a $f$, temos que

$$
\lim _{n \rightarrow \infty} f_{n}(x)=\lim _{n \rightarrow \infty} \Phi_{x}\left(f_{n}\right)=\Phi_{x}(f)=f(x)
$$

Portanto, $\left(f_{n}\right)_{n \in \mathbb{N}}$ converge pontualmente a $f$.

Reciprocamente, suponhamos que $\left(f_{n}\right)_{n \in \mathbb{N}}$ converge pontualmente a $f$ e é limitada. Tomemos $M>0$ tal que para todo $n \in \mathbb{N},\left\|f_{n}\right\| \leq M$. Temos que a função constante igual a $M$ é integrável com relação a toda $\mu \in C(K)^{*}$. Daí, pelo teorema anterior, temos que para toda $\mu \in C(K)^{*}$,

$$
\lim _{n \rightarrow \infty} \mu\left(f_{n}\right)=\lim _{n \rightarrow \infty} \int_{K} f_{n} d \mu=\int_{K} f d \mu=\mu(f) .
$$

Portanto, $\left(f_{n}\right)_{n \in \mathbb{N}}$ converge fracamente a $f$.

\subsection{A topologia fraca em $C(K)^{*}$}

Vejamos então como se comporta a topologia fraca em espaços da forma $C(K)^{*}$ :

Lema 3.16. Seja $K$ um espaço booleano e sejam $\left(\xi_{n}\right)_{n \in \mathbb{N}} \subseteq C(K)^{*}$. Considere $\left(\mu_{n}\right)_{n \in \mathbb{N}} \subseteq$ $\mathcal{M}(K)$ as medidas de Radon (dadas pelo Teorema de Representação de Riesz, Teorema 3.13) sobre $K$ tais que

$$
\forall f \in C(K) \quad \xi_{n}(f)=\int_{K} f d \mu_{n}
$$

Se existem $a \in \operatorname{Bor}(K), \varepsilon>0$ e $M_{1}, M_{2} \subseteq \mathbb{N}$ infinitos tais que

$$
\forall n \in M_{1}\left|\mu_{n}(a)\right| \geq \varepsilon \text { e } \forall n \in M_{2}\left|\mu_{n}(a)\right| \leq \frac{\varepsilon}{2},
$$

então $\left(\xi_{n}\right)_{n \in \mathbb{N}}$ não é convergente na topologia fraca. 
Demonstração. Suponha $a \in \operatorname{Bor}(K), \varepsilon>0$ e $M_{1}, M_{2} \subseteq \mathbb{N}$ como na hipótese. Defina $\Phi: C(K)^{*} \rightarrow \mathbb{R}$ por $\Phi(\mu)=\int_{K} \chi_{a} d \mu$.

$\Phi$ está bem definida, pois como $a \in \operatorname{Bor}(K)$, temos que $\chi_{a}$ é integrável com relação a toda medida de Radon $\mu$. É fácil ver que $\Phi$ é linear. $\Phi$ é limitada, pois dada $\mu \in C(K)^{*}$, temos

$$
\|\Phi(\mu)\|=\left|\int_{K} \chi_{a} d \mu\right|=|\mu(a)| \leq\|\mu\| .
$$

Portanto, temos que $\Phi \in C^{* *}(K)$. Daí, temos que

$$
\forall n \in M_{1}\left|\Phi\left(\xi_{n}\right)\right|=\left|\int_{K} \chi_{a} d \mu_{n}\right|=\left|\mu_{n}(a)\right| \geq \varepsilon
$$

e

$$
\forall n \in M_{2}\left|\Phi\left(\xi_{n}\right)\right|=\left|\int_{K} \chi_{a} d \mu_{n}\right|=\left|\mu_{n}(a)\right| \leq \frac{\varepsilon}{2} .
$$

Portanto, $\left(\Phi\left(\xi_{n}\right)\right)_{n \in \mathbb{N}}$ não é uma seqüência convergente em $\mathbb{R}$ e, portanto, pelo Lema $2.12,\left(\xi_{n}\right)_{n \in \mathbb{N}}$ não converge na topologia fraca.

O próximo resultado será utilizado para achar uma seqüência $\left(a_{n}\right)_{n \in \mathbb{N}}$ de abertosfechados de $K$ como acima. À primeira vista, podemos notar que se tivermos um conjunto limitado de medidas de Radon que não é relativamente fracamente compacto, ele nos fornece uma seqüência de abertos e não de abertos-fechados. Porém, ao trabalharmos com espaços booleanos, a partir desta seqüência de abertos, podemos obter uma de abertosfechados com as mesmas propriedades, usando a regularidade da medida (de Radon).

Teorema 3.17 (de Dieudonné-Grothendieck). Seja $K$ um espaço compacto Hausdorff e considere Bor $(K)$ a álgebra $\sigma$-completa dos subconjuntos borelianos de $K$. Seja $M$ um subconjunto limitado de medidas de Radon sobre $K$. Então, temos que $M$ é relativamente fracamente compacto se, e somente se, para toda seqüência $\left(a_{n}\right)_{n \in \mathbb{N}}$ de abertos de $K$ dois a dois disjuntos, tem-se que $\mu\left(a_{n}\right)$ converge uniformemente a 0 para $\mu \in M$.

Demonstração. Indicamos [Di], Teorema 14, página 98.

\subsection{A topologia fraca* em $C(K)^{*}$}

Por fim, vejamos como se comporta a topologia fraca* em espaços da forma $C(K)^{*}$ : 
Lema 3.18. Seja $K$ um espaço booleano e sejam $\left(\xi_{n}\right)_{n \in \mathbb{N}} \subseteq C(K)^{*}$. Considere $\left(\mu_{n}\right)_{n \in \mathbb{N}} \subseteq$ $\mathcal{M}(K)$ as medidas de Radon (dadas pelo Teorema de Representação de Riesz, Teorema 3.13) sobre $K$ tais que

$$
\forall f \in C(K) \quad \xi_{n}(f)=\int_{K} f d \mu_{n} .
$$

Se existem $a \in C l o p(K), \varepsilon>0$ e $M_{1}, M_{2} \subseteq \mathbb{N}$ infinitos tais que

$$
\forall n \in M_{1}\left|\mu_{n}(a)\right| \geq \varepsilon \text { e } \forall n \in M_{2}\left|\mu_{n}(a)\right| \leq \frac{\varepsilon}{2},
$$

então $\left(\xi_{n}\right)_{n \in \mathbb{N}}$ não é convergente na topologia fraca*.

Demonstração. Suponha $a \in C l o p(K), \varepsilon>0$ e $M_{1}, M_{2} \subseteq \mathbb{N}$ como na hipótese. Como $a \in C l o p(K)$, temos que $\chi_{a} \in C(K)$. Daí, temos que

$$
\forall n \in M_{1}\left|\xi_{n}\left(\chi_{a}\right)\right|=\left|\int_{K} \chi_{a} d \mu_{n}\right|=\left|\mu_{n}(a)\right| \geq \varepsilon
$$

e

$$
\forall n \in M_{2}\left|\xi_{n}\left(\chi_{a}\right)\right|=\left|\int_{K} \chi_{a} d \mu_{n}\right|=\left|\mu_{n}(a)\right| \leq \frac{\varepsilon}{2}
$$

Portanto, $\left(\xi_{n}\left(\chi_{a}\right)\right)_{n \in \mathbb{N}}$ não é uma seqüência convergente em $\mathbb{R}$ e então, pelo Lema 2.17 , $\left(\xi_{n}\right)_{n \in \mathbb{N}}$ não converge na topologia fraca*.

Lema 3.19. Seja A uma álgebra de Boole. Suponha $\left(\mu_{n}\right)_{n \in \mathbb{N}} \subseteq C(S(A))^{*}$ uma seqüência que converge na topologia fraca* a $\mu \in C(S(A))^{*}$ e que não converge na topologia fraca. Então, existem $\varepsilon>0,\left(a_{n}\right)_{n \in \mathbb{N}} \subseteq A$ anticadeia $e\left(k_{n}\right)_{n \in \mathbb{N}} \subseteq \mathbb{N}$ crescente tais que

$$
\forall n \in \mathbb{N} \quad\left|\mu_{k_{n}}\left(a_{n}\right)\right| \geq \varepsilon .
$$

Demonstração. Seja $K=S(A)$.

Se $\left\{\mu_{n}: n \in \mathbb{N}\right\}$ fosse fracamente compacto, pelo Teorema de Eberlein-Šmulian (2.15), seria fracamente seqüencialmente compacto. Daí, existiriam $M_{1}, M_{2} \subseteq \mathbb{N}$ infinitos disjuntos tais que $\left(\mu_{n}\right)_{n \in M_{i}}$ seria fracamente convergente a $\nu_{i}$, para $i=1,2$, e $\nu_{1} \neq \nu_{2}$. Como convergência fraca implica em convergência fraca*, teríamos que $\left(\mu_{n}\right)_{n \in M_{i}}$ seria fraca* convergente a $\nu_{i}$, para $i=1,2$, uma contradição.

Assim, podemos assumir que $\left\{\mu_{n}: n \in \mathbb{N}\right\}$ não é fracamente compacto. Pelo Princípio de Limitação Uniforme (2.6), $\left(\mu_{n}\right)_{n \in \mathbb{N}}$ é uma seqüência limitada. Pelo Teorema de Dieudonné-Grothendieck (3.17) existe uma seqüência $\left(U_{n}\right)_{n \in \mathbb{N}}$ de abertos de $K$ dois a dois 
disjuntos e $\varepsilon>0$ tais que para todo $n_{0} \in \mathbb{N}$, existe $n \geq n_{0}$ e $k_{n} \in \mathbb{N}$ tais que $\left|\mu_{k_{n}}\left(U_{n}\right)\right| \geq \varepsilon$. Como $K$ é um espaço booleano, usando a regularidade de cada $\mu_{n}$, podemos supor, sem perda de generalidade, que $U_{n}=a_{n}^{*}$, para $a_{n} \in A$. Além disso, se para um $k \in \mathbb{N}$ temos $\left|\mu_{k}\left(a_{n_{i}}^{*}\right)\right| \geq \varepsilon$ para uma seqüência $\left(n_{i}\right)_{i \in \mathbb{N}} \subseteq \mathbb{N}$, segue que

$$
\left|\mu_{k}\right|\left(\bigcup_{i \in \omega} a_{n_{i}}^{*}\right) \geq \sum_{i \in \omega}\left|\mu_{k}\left(a_{n_{i}}^{*}\right)\right|=\infty
$$

contradizendo que $\mu_{k}$ é limitada.

Assim, sejam $n_{0} \in \mathbb{N}$ e $k_{0} \in \mathbb{N}$ tais que $\left|\mu_{k_{0}}\left(a_{n_{0}}^{*}\right)\right| \geq \varepsilon$ e por indução, construímos $n_{i+1}>n_{i}$ e $k_{i+1}>k_{i}$ tais que $\left|\mu_{k_{i}}\left(a_{n_{i}}^{*}\right)\right| \geq \varepsilon$.

As seqüências $\left(a_{i_{n}}\right)_{n \in \mathbb{N}}$ e $\left(k_{n}\right)_{n \in \mathbb{N}}$ satisfazem a tese do lema.

Convém mencionar que os resultados apresentados nestas três últimas seções ajudarão na demonstração do fato que alguns espaços (dos Capítulos 5, 6 e 7) são de Grothendieck.

\subsection{Subespaços e subespaços complementados}

O objetivo desta seção é fazer uma análise dos subespaços e subespaços complementados de espaços $C(K)$. Começamos com exemplos gerais e passamos à análise do superespaços do $c_{0}$ e do $l_{\infty}$.

\subsubsection{Alguns exemplos}

Às vezes, ao trabalhar com espaços de Banach da forma $C(K)$, precisamos estender funções contínuas a valores reais de um subespaço $L$ ao espaço $K$. Porém, o Teorema de Extensão de Tietze (1.36) não é suficiente para isso, pois quando trabalhamos com espaços de Banach, estamos preocupados com a linearidade da aplicação que levará $C(L)$ em $C(K)$. Assim, sendo $L \subseteq K$, não teremos necessariamente uma cópia de $C(L)$ em $C(K)$. Vejamos um caso em que isso ocorre:

Exemplo 3.20. Seja $K$ um espaço compacto Hausdorff e suponha que $U \subseteq K$ é um aberto-fechado. Então temos que $C(U)$ é isomorfo a um subespaço complementado de $C(K)$. 
Demonstração. Considere $T: C(U) \longrightarrow C(K)$ dada por

$$
T(f)=g \quad \text { onde }\left.g\right|_{U}=f \text { e }\left.g\right|_{(K \backslash U)}=0 .
$$

É fácil ver que $T$ é um isomorfismo sobre sua imagem. $\operatorname{Logo}, \operatorname{Im}(T) \subseteq C(K)$ é isomorfo a $C(U)$. Por abuso de linguagem diremos que $C(U) \subseteq C(K)$. Então a função restrição $P: C(K) \rightarrow \operatorname{Im}(T)$ definida por $P(f)=T\left(\left.f\right|_{U}\right)$ é uma projeção: é um operador linear limitado (claro), é sobrejetora e $P^{2}=P$ pois dada $h \in C(U), P(T(h))=T\left(\left.T(h)\right|_{U}\right)=$ $T(h)$.

Por outro lado, não precisamos que $L$ seja subespaço de $K$ como mostra o seguinte:

Exemplo 3.21. Se $K, L$ são espaços compactos Hausdorff e $F: K \rightarrow L$ é uma função contínua sobrejetora, então $C(L)$ é isométrico a um subespaço de $C(K)$.

Demonstração. Considere $T: C(L) \rightarrow C(K)$ definida por $T(f)=f \circ F$. T está bem definida, pois $F$ é contínua. É fácil ver que $T$ é linear. Se $f \in C(L)$, temos que

$$
\|T(f)\|=\|f \circ F\|=\sup _{x \in K}|f \circ F(x)|=\sup _{x \in L}|f(x)|=\|f\|,
$$

pois $F$ é sobrejetora. Logo, $T$ é injetora e, portanto, é uma isometria de $C(L)$ sobre $T[C(L)] \subseteq C(K)$.

Vejamos que no caso de retratos, isto também acontece, de forma ainda mais forte:

Exemplo 3.22. Sejam $L \subseteq K$ espaços compactos Hausdorff e suponha $r: K \rightarrow L$ um retrato. Então $C(L)$ é isométrico a um subespaço complementado em $C(K)$.

Demonstração. Como $r$ é retrato, temos que é contínua, sobrejetora e $\left.r\right|_{L}=i d_{L}$. Já sabemos, do exemplo anterior, que $C(L)$ é isométrico ao subespaço $X=\{f \circ r: f \in C(L)\}$ de $C(K)$.

Definamos $P: C(K) \rightarrow X$ por $P(f)=\left.f\right|_{L} \circ r$. É claro que $P$ está bem definida e que é linear. Além disso, dada $f \in C(K)$, temos

$$
\|P(f)\|=\left\|\left.f\right|_{L} \circ r\right\|=\sup _{x \in K}|f|_{L}(r(x))\left|=\sup _{x \in L}\right| f(x) \mid \leq\|f\|,
$$

uma vez que $r$ é sobrejetora. Assim, $P$ é limitada. 
$P$ é sobrejetora, pois dada $g \in X$, temos que existe $f \in C(L)$ tal que $g=f \circ r$. Daí, pelo Teorema da Extensão de Tietze (1.36), existe $\tilde{f} \in C(K)$ tal que $\left.\tilde{f}\right|_{L}=f$. Logo, $g=f \circ r=\left.\tilde{f}\right|_{L} \circ r=P(\tilde{f})$.

Por fim, dada $f \in C(K)$ e $x \in K$, como $r(x) \in L$, temos que $r(r(x))=r(x)$ e então, $P(P(f))(x)=P\left(\left.f\right|_{L} \circ r\right)(x)=\left.\left(\left.f\right|_{L} \circ r\right)\right|_{L}(r(x))=\left.f\right|_{L}(r(r(x)))=\left.f\right|_{L}(r(x))=P(f)(x)$.

Logo, $P^{2}=P$ e, portanto, $P$ é uma projeção de $C(K)$ sobre $X$.

Por fim, vejamos que se $L \subseteq K$, então $C(L)$ é um quociente de $C(K)$.

Teorema 3.23. Sejam $L \subseteq K$ compactos Hausdorff e defina $C_{0}(K \mid L)=\{f \in C(K)$ : $\left.\left.f\right|_{L}=0\right\}$. Daí,

$$
C(L) \equiv C(K) / C_{0}(K \mid L)
$$

Demonstração. Considere $T: C(K) \rightarrow C(L)$ dada por $T(f)=\left.f\right|_{L}$. É claro que $T$ está bem definida e é fácil ver que $T$ é linear. Pelo Teorema de Extensão de Tietze, garantimos que $T$ é sobrejetora.

Daí, pela Proposição 2.38, $i: C(K) / K e r(T) \rightarrow C(L)$ dado por $i([f])=T(f)$ é um isomorfismo. Além disso, é fácil ver que $\operatorname{Ker}(T)=C_{0}(K \mid L)$. Vejamos que é uma isometria.

Fixemos $f \in C(K)$. Dada $g \in C_{0}(K \mid L)$, temos que

$$
\|i([f])\|=\|T(f)\|=\left\|\left.f\right|_{L}\right\| \leq\|f-g\| .
$$

Logo, $\|i([f])\| \leq\|[f]\|$. Por outro lado, pelo Teorema de Extensão de Tietze, existe $f^{\prime} \in C(K)$ tal que $\left.f^{\prime}\right|_{L}=\left.f\right|_{L}$ e $\left\|f^{\prime}\right\|=\left\|\left.f\right|_{L}\right\|$. Daí,

$$
\|[f]\| \leq\left\|f^{\prime}\right\|=\left\|\left.f\right|_{L}\right\|=\|i([f])\| .
$$

Portanto, $i$ é uma isometria.

\subsubsection{O $c_{0}$ como subespaço}

Após alguns exemplos de subespaços complementados em $C(K)$, pretendemos agora estudar os espaços de Banach que possuem cópias de $c_{0}$, complementadas ou não. $\mathrm{O}$ primeiro 
resultado importante, o Teorema de Sobczyk, garante que $c_{0}$ é complementado em todo seu superespaço separável. Para prová-lo, precisamos do seguinte lema:

Lema 3.24. Seja $X$ um espaço de Banach separável e seja $Y$ um subespaço fechado de $X$. Se $T_{0}: Y \rightarrow c_{0}$ é linear e limitada com $\left\|T_{0}\right\| \leq \lambda$, para um $\lambda>0$, então existe $T: X \rightarrow c_{0}$ linear e limitada tal que $\left.T\right|_{Y}=T_{0}$ e $\|T\| \leq 2 \lambda$.

Demonstração. Considere $B_{\lambda}=\left\{f \in X^{*}:\|f\| \leq \lambda\right\}$. Pelo Teorema de Alaoglu (Teorema $2.19)$, temos que $B_{\lambda}$ é fracamente* compacto.

Como $X$ é separável, segue que a bola unitária de $X^{*}, B_{X^{*}}$ é metrizável. Portanto, $B_{\lambda}$ é metrizável. Seja $d$ uma métrica sobre $B_{\lambda}$.

Para cada $n \in \mathbb{N}$, defina $\Phi_{n}: Y \rightarrow \mathbb{R}$ por $\Phi_{n}(y)=T_{0}(y)(n)$, para todo $y \in Y$. É fácil ver que cada $\Phi_{n}$ é linear. Além disso, dado $y \in Y$, temos

$$
\left\|\Phi_{n}(y)\right\| \leq\left\|T_{0}(y)\right\| \leq\left\|T_{0}\right\| \cdot\|y\| \leq \lambda\|y\| .
$$

Logo, cada $\Phi_{n} \in Y^{*}$. Daí, pelo Teorema de Hahn-Banach, existe, para cada $n \in \omega$, $\tilde{\Phi}_{n} \in X^{*}$ tal que $\left.\tilde{\Phi}_{n}\right|_{Y}=\Phi_{n}$ e $\left\|\tilde{\Phi}_{n}\right\|=\|\Phi\| \leq \lambda$. Portanto, $\left(\tilde{\Phi}_{n}\right)_{n \in \omega} \subseteq B_{\lambda}$.

Considere $K=B_{\lambda} \cap\left\{f \in X^{*}: \forall y \in Y f(y)=0\right\}$. Temos que todo ponto de acumulação de $\left(\tilde{\Phi}_{n}\right)_{n \in \omega} \subseteq B_{\lambda}$ pertence a $K$. Daí, $d\left(\tilde{\Phi}_{n}, K\right) \rightarrow 0$ quando $n \rightarrow \infty$.

Como $K$ é fechado, para cada $n \in \omega$, existe $\Psi_{n} \in K$ tal que $d\left(\tilde{\Phi}_{n}, K\right)=d\left(\tilde{\Phi}_{n}, \Psi_{n}\right)$. Daí, 0 é o único ponto aderente a $\left\{\Phi_{n}-\Psi_{n}: \Phi_{n}-\Psi_{n} \in B_{2 \lambda}\right\}$.

Definimos $T(x)(n)=\tilde{\Phi}_{n}(x)-\Psi_{n}(x)$, para cada $x \in X$ e cada $n \in \omega$. Para ver que $T: X \rightarrow c_{0}$ está bem definida, considere $x \in X$ e temos

$$
\lim _{n \rightarrow \infty} T(x)(n)=\lim _{n \rightarrow \infty}\left(\tilde{\Phi}_{n}(x)-\Psi_{n}(x)\right) \leq\|x\| \lim _{n \rightarrow \infty}\left\|\tilde{\Phi}_{n}-\Psi_{n}\right\|=0 .
$$

É claro que $T$ é linear, uma vez que $\tilde{\Phi}_{n}$ e $\Psi$ o são. Vejamos que $T$ é limitada: dado $x \in X$ temos

$$
\|T(x)\|=\sup _{n \in \omega}\|T(x)(n)\|=\sup _{n \in \omega}\left\|\tilde{\Phi}_{n}(x)-\Psi_{n}(x)\right\| \leq \sup _{n \in \omega}\left\|\tilde{\Phi}_{n}-\Psi_{n}\right\| \cdot\|x\| \leq 2 \lambda\|x\|,
$$

e, portanto, $\|T\| \leq 2 \lambda$. Assim, temos o resultado.

Teorema 3.25 (de Sobczyk). Se $X$ é um espaço de Banach separável e $c_{0}$ é subespaço de $X$, então c co é complementado em $X$. 
Demonstração. Seja $c_{0} \subseteq X, X$ separável. Temos que $I d: c_{0} \rightarrow c_{0}$ é linear e limitada. Logo, existe $P: X \rightarrow c_{0}$ linear e limitada que estende $I d$, pelo lema anterior. Portanto, $P$ é uma projeção de $X$ sobre $c_{0}$.

Provemos agora o Lema de Philips, primeiro passo na direção da descoberta de que o $l_{\infty}$ é primo (fato que será mostrado no Capítulo 5). Apresentamos tal resultado aqui, pois ele ilustra que o Teorema de Sobczyk não pode ser generalizado para espaços não separáveis.

Proposição 3.26 (Philips). A cópia standard de c nấo é complementada no $l_{\infty}$.

Demonstração. Suponhamos, por absurdo, que existe $X \subseteq l_{\infty}$ tal que $l_{\infty}=c_{0} \oplus X$. Seja $P$ a projeção de $l_{\infty}$ sobre $X$.

Considere $\left(a_{\xi}\right)_{\xi<\omega_{1}} \subseteq \wp(\mathbb{N})$ uma família quase disjunta ${ }^{5}$. Daí, existem $\delta>0, k \in \mathbb{N}$ e $S \subseteq \omega_{1}$ tais que $|S|=\omega_{1}$ e para todo $\xi \in S$, tem-se que $\left|P\left(\chi_{a_{\xi}}\right)(k)\right| \geq \delta$. Podemos supor, sem perda de generalidade, que $P\left(\chi_{a_{\xi}}\right)(k) \geq \delta$ para todo $\xi \in S$.

Daí, para $\xi_{1}, \ldots, \xi_{n} \in S$, existem $F_{1}, \ldots F_{n} \subseteq \mathbb{N}$ finitos tais que

$$
\chi_{\bigcup_{i=1}^{n} a_{\xi_{i}}}=\sum_{i=1}^{n}\left(\chi_{a_{\xi_{i}}}-\chi_{F_{i}}\right) .
$$

Como $\left\|\chi \bigcup_{i=1}^{n} a_{\xi_{i}}\right\|=1$, temos que

$$
\left\|P\left(\chi_{\bigcup_{i=1}^{n} a_{\xi_{i}}}\right)\right\| \leq\|P\| .
$$

Por outro lado,

$$
\left\|P\left(\chi_{\bigcup_{i=1}^{n} a_{\xi_{i}}}\right)\right\|=\left\|P\left(\sum_{i=1}^{n}\left(\chi_{a_{\xi_{i}}}-\chi_{F_{i}}\right)\right)\right\|=\left\|P\left(\sum_{i=1}^{n} \chi_{a_{\xi_{i}}}\right)\right\| \geq P\left(\sum_{i=1}^{n} \chi_{a_{\xi_{i}}}\right)(k) \geq n \cdot \delta
$$

uma contradição.

Vejamos então que para todo $K$ booleano, $C(K)$ tem uma cópia de $c_{0}$. Para isto, notemos primeiramente o seguinte:

\footnotetext{
${ }^{5}$ Dizemos que $\mathcal{F} \subseteq \wp(\mathbb{N})$ é quase disjunta se, para quaisquer $F_{1}, F_{2} \in \mathcal{F}$, tem-se que $F_{1} \cap F_{2}$ é finito. Ao provarmos, no Capítulo 6 , que $\wp(\mathbb{N}) / F$ in tem uma anticadeia de cardinalidade $2^{\omega}$, garantimos que existe uma família quase disjunta de cardinalidade $\omega_{1}$.
} 
Exemplo 3.27. $C(S(F$ in $\operatorname{Cofin}(\mathbb{N}))) \sim c_{0}$.

Demonstração. Seja $S($ FinCofin $(\mathbb{N}))=\left\{x_{n}: n \in \mathbb{N}\right\} \cup\{\infty\}$ um conjunto infinito discreto com um único ponto de acumulação, isto é, uma seqüência convergente com seu limite. Defina $T: C(S($ FinCofin $(\mathbb{N}))) \rightarrow c_{0}$ por $T(f)(0)=f(\infty)$ e $T(f)(n+1)=f\left(x_{n}\right)-f(\infty)$. $T$ está bem definida, uma vez que $f\left(x_{n}\right)$ converge a $f(\infty)$.

$T$ é linear, pois é definida pontualmente. Além disso, é fácil ver que $\|T(f)\| \leq 2\|f\|$. $T$ é injetora, pois se $T(f)=0$, então $f(\infty)=0$ e daí, $f\left(x_{n}\right)=T(f)(n+1)+f(\infty)=$ $T(f)(n+1)=0$.

Por fim, $T$ é sobrejetora, pois dada $\left(\alpha_{n}\right)_{n \in \mathbb{N}} \in c_{0}$, definimos $f(\infty)=\alpha_{0}$ e $f\left(x_{n}\right)=$ $\alpha_{n+1}+\alpha_{0}$ e temos que $T(f)=\left(\alpha_{n}\right)_{n \in \mathbb{N}}$. Daí, pelo Teorema da Aplicação Aberta (2.7), temos que $T$ é um isomorfismo.

Proposição 3.28. Para todo espaço booleano ${ }^{6}$ infinito $K, C(K)$ possui um subespaço isomorfo a $c_{0}$.

Demonstração. O resultado vale para qualquer $K$ compacto Hausdorff e infinito. Porém, faremos a demonstração apenas para espaços $K$ booleanos. Como $K$ é infinito, temos que $C l o p(K)$ é infinita. Podemos tomar então $\left(a_{n}\right)_{n \in \mathbb{N}} \subseteq C l o p(K)$ uma anticadeia. Considere $A=\left\langle\left\{a_{n}: n \in \mathbb{N}\right\}\right\rangle$ subálgebra de $C \operatorname{lop}(K)$. Vejamos que $A$ é isomorfa a $\operatorname{Fin} C o f i n(\mathbb{N})$ : defina $h: A \rightarrow \operatorname{FinCofin}(\mathbb{N})$ por $h(a)=\left\{n \in \mathbb{N}: a_{n} \leq a\right\}$. É fácil ver que $h$ é um homomorfismo.

Temos que $h$ é injetor, pois se $h(a)=0$, para um $a \in A$, temos, pelo Teorema 1.8, que $a=\sum_{i=1}^{n}\left(\prod_{j_{i}=1}^{k_{i}}\left(A_{i, j_{i}}\right)\right)$, onde cada $A_{i, j_{i}}=a_{n}$, ou $A_{i, j_{i}}=-a_{n}$. Daí, temos que para todo $1 \leq i \leq n$,

$$
\left\{n \in \mathbb{N}: \forall j_{i} 1 \leq j_{i} \leq k_{i} a_{n} \leq A_{i, j_{i}}\right\}=\prod_{j_{i}=1}^{k_{i}} h\left(A_{i, j_{i}}\right)=h\left(\prod_{j_{i}=1}^{k_{i}}\left(A_{i, j_{i}}\right)\right)=0 .
$$

Logo, cada $\prod_{j_{i}=1}^{k_{i}} A_{i, j_{i}}=0$ e, portanto, $a=0$.

$h$ é sobrejetor, pois para cada $F \subseteq \mathbb{N}$ finito, tomando $a_{F}=\bigcup_{n \in F} a_{n} \in A$, temos que $h\left(a_{F}\right)=F$. Logo, se $X \subseteq \mathbb{N}$ é cofinito, então $X=h\left(a_{\mathbb{N} \backslash X}\right)$.

\footnotetext{
${ }^{6} \mathrm{Na}$ realidade esta proposição vale para todo espaço compacto Hausdorff $K$ infinito. Como comentamos na introdução deste capítulo, não faremos sua demonstração em geral, uma vez que ela é mais técnica e não nos interessa neste trabalho.
} 


\section{ESPAÇOS DE BANACH DA FORMA $C(K)$}

Assim, $C l o p(K)$ tem subálgebra isomorfa a FinCofin $(\mathbb{N})$ e, portanto, $K$ tem $S(A)$ como imagem homomorfa. Do Exemplo 3.21 e do exemplo anterior, temos que $C(K)$ tem subespaço isométrico a $C(S(A)) \sim c_{0}$.

Obtemos então o seguinte resultado, ilustrando que para $C(K)$ separável, considerar quando $c_{0}$ é subespaço complementado não fornece informações, pois isso sempre acontece: Corolário 3.29. Seja $K$ um espaço booleano ${ }^{7}$ infinito. Se $C(K)$ é um espaço de Banach separável, então $C(K)$ possui uma cópia complementada de $c_{0}$.

Pela Proposição 3.4, temos que, para $K$ booleano, $C(K)$ ser separável é equivalente a $K$ ter peso $\omega$. Assim, temos o primeiro exemplo de que propriedades do espaço $K$ influenciam os subespaços (complementados) de $C(K)$. Vejamos que podemos generalizar o corolário acima, usando outra propriedade de $C(K)$ :

Teorema 3.30. Seja $K$ um espaço booleano ${ }^{8}$. Se $K$ possui uma seqüência convergente não trivial, então $C(K)$ possui uma cópia complementada de $c_{0}$.

Demonstração. Suponha $K$ booleano e $\left(x_{n}\right)_{n \in \mathbb{N}} \subseteq K$ e $x \in K$ tal que $x_{n}$ converge para $x$. Seja $X=\left\{x_{n}: n \in \mathbb{N}\right\} \cup\{x\}$. Podemos obter $\left(b_{n}\right)_{n \in \mathbb{N}} \subseteq C l o p(K)$ tais que $b_{n} \cap X=\left\{x_{n}\right\}$. Tomemos então $a_{0}=b_{0}$ e $a_{n+1}=b_{n+1} \backslash \bigcup_{i \leq n} a_{i}$. Temos assim que $\left(a_{n}\right)_{n \in \mathbb{N}} \subseteq C \operatorname{Cop}(K)$ é uma anticadeia e $a_{n} \cap X=\left\{x_{n}\right\}$.

Definamos $f: K \rightarrow X$ por

$$
f(y)=\left\{\begin{array}{cl}
x_{n}, & \text { se } y \in a_{n} \\
x, & \text { se } y \notin \bigcup_{n \in \mathbb{N}} a_{n} .
\end{array}\right.
$$

Vejamos que $f$ é um retrato. $f$ é contínua, pois se $U \subseteq X$ é um aberto, então ou $U=\left\{x_{n_{1}}, \ldots x_{n_{k}}\right\}$ ou $U=X \backslash\left\{x_{n_{1}}, \ldots x_{n_{k}}\right\}$. Daí, ou $f^{-1}[U]=a_{n_{1}} \cup \cdots \cup a_{n_{k}}$, ou $f^{-1}[U]=K \backslash a_{n_{1}} \cup \cdots \cup a_{n_{k}}$. Como ambos são abertos-fechados, temos que $f$ é contínua.

Além disso, $f\left(x_{n}\right)=x_{n}$ e $f(x)=x$. Logo, $\left.f\right|_{X}=i d$. Portanto, $f$ é um retrato e, pelo Exemplo 3.22, temos que $C(X)$ é complementado em $C(K)$. Mas pelo Exemplo 3.27, temos que $C(X) \sim c_{0}$. Daí, $C(K)$ tem uma cópia complementada de $c_{0}$.

\footnotetext{
${ }^{7}$ Temos aqui o mesmo caso que na proposição anterior. O resultado vale para qualquer $K$ compacto Hausdorff infinito.

${ }^{8}$ Temos aqui mais um resultado que vale para $K$ compacto Hausdorff e que provamos apenas para $K$ booleano.
} 
Convém notar que a volta não é verdadeira, isto é, existe $K$ booleano sem seqüências convergentes não triviais tal que $c_{0}$ é subespaço complementado de $C(K)$ (veja Exemplo 4.10 de [Scha]).

\subsubsection{O $l_{\infty}$ como subespaço}

Para provar que o $l_{\infty}$ é complementado em todo seu superespaço, vejamos primeiramente a definição de um caso especial de subespaços complementados:

Definição 3.31. Dizemos que um espaço de Banach $X$ é injetivo se quaisquer que sejam $Y, Z$ espaços de Banach em que $Y$ é subespaço de $Z$ e qualquer que seja $T_{0}: Y \rightarrow X$ uma aplicação linear e limitada, existe uma aplicação linear e limitada $T: Z \rightarrow X$ que estende $T$.

Temos o seguinte teorema, que traduz uma propriedade de álgebras de Boole a uma propriedade de espaços $C(K)$ :

Teorema 3.32 (de Nachbin-Goodman). Se K é um espaço extremamente desconexo ${ }^{9}$, então $C(K)$ é injetivo. Portanto, se A é completa, então $C(S(A))$ é injetivo.

Demonstração. Seja $K$ extremamente desconexo. Temos que $C l o p(K)$ é subálgebra de $\wp(K)$. Logo, pela Proposição $1.69, K$ é retrato de $S(\wp(K))$. Daí, pelo Exemplo 3.22, temos que $C(K)$ é complementado em $C(S(\wp(K))) \equiv l_{\infty}[K]$, sendo que $l_{\infty}[K]$ é o espaço das funções limitadas sobre $K$ (não faremos esta isometria, mas ela é análoga à isometria $l_{\infty} \equiv C(\beta \mathbb{N})$ apresentada no Capítulo 4$)$.

Vejamos que é suficiente provar que $l_{\infty}[K]$ é injetivo: suponha $X, Y$ espaços de Banach, $Y$ subespaço de $X$ e $T: Y \rightarrow C(K) \subseteq l_{\infty}[K]$. Se $l_{\infty}[K]$ é injetivo, então existe $\tilde{T}: X \rightarrow$ $l_{\infty}[K]$ aplicação linear limitada que estende $T$. Considere $P: l_{\infty}[K] \rightarrow C(K)$ uma projeção. Então, temos que $P \circ \tilde{T}: X \rightarrow C(K)$ é uma aplicação linear limitada que estende $T$ e, portanto, $C(K)$ é injetivo.

Para mostrar que $l_{\infty}[K]$ é injetivo, considere $T: Y \rightarrow l_{\infty}[K]$ uma aplicação linear limitada. Temos que $T(y)=(T(y)(\xi))_{\xi \in K}$. Defina, para cada $\xi \in K, \Phi_{\xi}: Y \rightarrow \mathbb{R}$ por $\Phi_{\xi}(y)=T(y)(\xi)$. É fácil ver que cada $\Phi_{\xi} \in Y^{*}$. Daí, pelo Teorema de Hahn-Banach

\footnotetext{
${ }^{9}$ Todo espaço extremamente desconexo é booleano, mas não provaremos isto aqui.
} 
(2.5), existe $\tilde{\Phi}_{\xi}: X \rightarrow \mathbb{R}$ funcional linear contínua que estende $\Phi_{\xi}$ e tal que $\left\|\Phi_{\xi}\right\|=\left\|\tilde{\Phi}_{\xi}\right\|$. Defina $\tilde{T}: X \rightarrow l_{\infty}[K]$ por $T(x)(\xi)=\tilde{\Phi}_{\xi}(x)$. Temos que $\tilde{T}$ assim definida é linear e

$$
\left.\|\tilde{T}\|=\sup _{x \in X} \sup _{\xi \in K}|\tilde{T}(x)(\xi)|\right)=\sup _{x \in X}\left(\sup _{\xi \in K}\left|\tilde{\Phi}_{\xi}(x)\right|\right)=\sup _{\xi \in K}\left\|\tilde{\Phi}_{\xi}\right\|=\sup _{\xi \in K}\left\|\Phi_{\xi}\right\|=\|T\| .
$$

Portanto, $l_{\infty}[K]$ é injetivo.

Pelo teorema acima, parece-nos que ser injetivo é uma propriedade relativamente comum entre os espaços de Banach $C(K)$. Porém, não se conhece outros espaços injetivos além dos extremamente desconexos, nem mesmo da forma $C(K)$ para $K$ compacto Hausdorff, não booleano.

Vamos agora analisar quais espaços de Banach devem ter o $l_{\infty}$ como subespaço complementado. O resultado que temos é um corolário da seguinte proposição:

Proposição 3.33. $l_{\infty}$ é injetivo.

Demonstração. Segue diretamente do teorema anterior e do Exemplo 1.25 e do fato que $l_{\infty} \equiv C(S(\wp(\mathbb{N}))$, como veremos no Capítulo 5 (Teorema 5.3).

Note que esta proposição é uma espécie de Teorema de Hahn-Banach, com o espaço $l_{\infty}$ no lugar do corpo.

Corolário 3.34. Se $X$ é um espaço de Banach e $l_{\infty}$ é subespaço de $X$, então $l_{\infty}$ é complementado em $X$.

Demonstrą̧ão. Considere $I d: l_{\infty} \rightarrow l_{\infty}$. Pelo lema anterior, temos que existe $P: X \rightarrow l_{\infty}$ linear e limitada tal que $\left.P\right|_{l_{\infty}}=I d$ e $\|P\|=1$. Logo, $l_{\infty}$ é complementado em $X$.

\subsection{Lemas combinatórios}

Apresentamos nesta seção, dois lemas combinatórios. O primeiro deles será utilizado na demonstração que alguns espaços dos Capítulos 5, 6 e 7 têm a propriedade de Grothendieck:

Lema 3.35. Sejam A uma álgebra de Boole e $K=S(A)$. Seja $\left(a_{n}\right)_{n \in \omega} \subseteq A$ uma anticadeia e $\left(\mu_{k}\right)_{k \in \mathbb{N}} \subseteq C(K)^{*}$. Se $\left(N_{\xi}\right)_{\xi<\omega_{1}} \subseteq \wp(\mathbb{N})$ é uma família quase disjunta ${ }^{10}$ de conjuntos

\footnotetext{
${ }^{10}$ Lembramos que a existência de tal família será provada no Capítulo 6 .
} 
infinitos, então existe $\xi<\omega_{1}$ tal que

$$
\mu_{k}\left(\chi_{\sum_{n \in M} a_{n}}\right)=\sum_{n \in M} \mu_{k}\left(\chi_{a_{n}}\right)
$$

para todo $k \in \mathbb{N}$ e todo $M \subseteq N_{\xi}$ tal que $\sum_{n \in M} a_{n} \in A$.

Demonstração. Considere uma família quase disjunta $\left(N_{\xi}: \xi<\omega_{1}\right)$ de subconjuntos infinitos de $\mathbb{N}$. Suponha por absurdo que o lema não é verdadeiro. Então, para cada $\xi<\omega_{1}$ existem $k_{\xi} \in \mathbb{N}$ e $M_{\xi} \subseteq N_{\xi}$ infinito tal que

$$
\sum_{i \in M_{\xi}} \mu_{k_{\xi}}\left(\chi_{a_{i}}\right) \neq \mu_{k_{\xi}}\left(\chi_{\sum_{i \in M_{\xi}} a_{i}}\right) \text { e } \sum_{i \in M_{\xi}} a_{i} \in A .
$$

Como temos $\omega$ possibilidades para todos os $k_{\xi}$ 's, podemos supor, sem perda de generalidade, que existe $k \in \mathbb{N}$ tal que para todo $\xi<\omega_{1}$, existe $M_{\xi} \subseteq N_{\xi}$ infinito tal que

$$
\sum_{i \in M_{\xi}} \mu_{k}\left(\chi_{a_{i}}\right) \neq \mu_{k}\left(\chi_{\sum_{i \in M_{\xi}} a_{i}}\right) \text { e } \sum_{i \in M_{\xi}} a_{i} \in A .
$$

Daí, para todo $\xi<\omega_{1}$ existe $m_{\xi} \in \mathbb{N}$ tal que

$$
\left|\mu_{k}\left(\chi_{\sum_{i \in M_{\xi}} a_{i}}\right)-\sum_{i \in M_{\xi}} \mu_{k}\left(\chi_{a_{i}}\right)\right| \geq \frac{1}{m_{\xi}} .
$$

Novamente, temos $\omega$ possibilidade para todos os $m_{\xi}$ 's e então, podemos supor, sem perda de generalidade, que existe $m \in \mathbb{N}$ tal que para todo $\xi<\omega_{1}$,

$$
\left|\mu_{k}\left(\chi_{\sum_{i \in M_{\xi}} a_{i}}\right)-\sum_{i \in M_{\xi}} \mu_{k}\left(\chi_{a_{i}}\right)\right| \geq \frac{1}{m} .
$$

Seja $\varepsilon=\frac{1}{m}$. Podemos assumir ainda, sem perda de generalidade, que todos os números acima têm o mesmo sinal, por exemplo, positivo (o caso negativo é análogo). Como

$$
\sum_{i \in M_{\xi}} \mu_{k}\left(\chi_{a_{i}}\right)=\lim _{l \rightarrow \infty} \sum_{i \in M_{\xi} \cap[l]} \mu_{k}\left(\chi_{a_{i}},\right)
$$

onde $[l]=\{0, \ldots, l\}$, temos que para cada $\xi<\omega_{1}$, existem $l \in \mathbb{N}$ arbitrariamente grandes tais que

$$
\mu_{k}\left(\chi_{\sum_{i \in M_{\xi} \backslash[l]} a_{i}}\right)=\mu_{k}\left(\chi_{\sum_{i \in M_{\xi}} a_{i}}\right)-\sum_{i \in M_{\xi} \cap[l]} \mu_{k}\left(\chi_{a_{i}}\right) \geq \varepsilon .
$$


Sejam $n \in \mathbb{N}$ tal que $n \cdot \varepsilon>\left\|\mu_{k}\right\|$ e $\xi_{1}<\cdots<\xi_{n}<\omega_{1}$ ordinais quaisquer. Sejam $l_{\xi_{i}} \in \mathbb{N}$ satisfazendo $(*)$ e tais que $M_{\xi_{i}} \backslash\left[l_{\xi_{i}}\right]$ são dois a dois disjuntos para $1 \leq i \leq n$. Temos então que

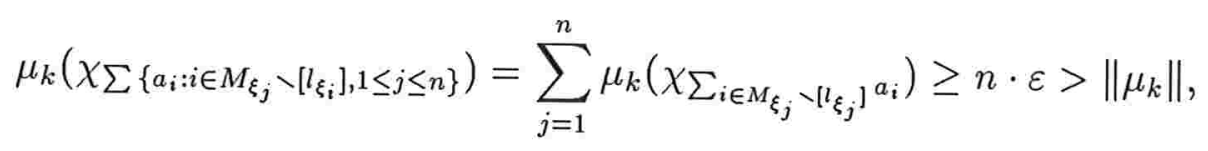

que é um absurdo. Logo, vale o lema.

O próximo resultado é, como o anterior, um resultado combinatório sobre números reais. Porém, sua maior aplicação neste trabalho se dá quando as seqüências de números reais de seu enunciado são seqüências de medidas aplicadas em abertos-fechados. Vejamos sua demonstração:

Teorema 3.36 (de Rosenthal). Seja, para cada $k \in \omega,\left(m_{n}^{k}\right)_{n \in \omega}$ uma seqüência e $M \in \mathbb{R}$ fixo tal que $\sum_{n \in \mathbb{N}}\left|m_{n}^{k}\right| \leq M$ para todo $k \in \mathbb{N}$. Então para todo $\varepsilon>0$ existe um subconjunto infinito $L$ de $\mathbb{N}$ tal que para todo $k \in L$

$$
\sum_{n \in L \backslash\{k\}}\left|m_{n}^{k}\right|<\varepsilon
$$

Demonstração. Fixe $\varepsilon>0$ e seja $\left(A_{i}\right)_{i \in \mathbb{N}}$ uma família de subconjuntos infinitos de $\mathbb{N}$ dois a dois disjuntos, tais que $\mathbb{N}=\bigcup_{i \in \mathbb{N}} A_{i}$. Se para algum $A_{i}$ vale que para todo $k \in A_{i}$ tem-se

$$
\sum_{n \in A_{i} \backslash\{k\}}\left|m_{n}^{k}\right|<\varepsilon
$$

então temos o resultado. Senão, para cada $i \in \omega$ existe $k_{i} \in A_{i}$ tal que

$$
\sum_{n \in A_{i} \backslash\left\{k_{i}\right\}}\left|m_{n}^{k_{i}}\right| \geq \varepsilon
$$

Seja $X_{1}$ o conjunto de tais $k_{i}$ e temos

$$
\begin{aligned}
\sum_{n \in X_{1}}\left|m_{n}^{k}\right| & =\left(\sum_{n \in X_{1}}\left|m_{n}^{k}\right|+\sum_{n \in \mathbb{N} \backslash X_{1}}\left|m_{n}^{k}\right|\right)-\sum_{n \in \mathbb{N} \backslash X_{1}}\left|m_{n}^{k}\right| \\
& =\sum_{n \in \mathbb{N}}\left|m_{n}^{k}\right|-\sum_{n \in \mathbb{N} \backslash X_{1}}\left|m_{n}^{k}\right| \leq M-\varepsilon
\end{aligned}
$$

para todo $k \in X_{1}$. Repita a construção acima para o conjunto $X_{1}$ e as seqüências $\left(m_{n}^{k}\right)_{n \in X_{1}}$, com $k \in X_{1}$, isto é, seja $\left(A_{i}^{1}\right)_{i \in \omega}$ uma família de subconjuntos infinitos de 
$X_{1}$ dois a dois disjuntos tais que $X_{1}=\bigcup_{i \in \omega} A_{i}^{1}$. Daí, teremos que ou existe $i \in \omega$ tal que para todo $n \in A_{i}^{1}$ tem-se

$$
\sum_{n \in A_{i}^{1} \backslash\{k\}}\left|m_{n}^{k}\right|<\varepsilon
$$

e temos o resultado, ou acharemos um subconjunto infinito $X_{2}$ de $X_{1}$ tal que

$$
\sum_{n \in X_{2}}\left|m_{n}^{k}\right| \leq M-2 \varepsilon
$$

para todo $k \in X_{2}$. Suponhamos que $X_{m}$ é um subconjunto infinito de $X_{m-1}$ tal que

$$
\begin{aligned}
\sum_{n \in X_{m}}\left|m_{n}^{k}\right| & =\left(\sum_{n \in X_{m}}\left|m_{n}^{k}\right|+\sum_{n \in \mathbb{N} \backslash X_{m}}\left|m_{n}^{k}\right|\right)-\sum_{n \in \mathbb{N} \backslash X_{m}}\left|m_{n}^{k}\right| \\
& =\sum_{n \in \mathbb{N}}\left|m_{n}^{k}\right|-\sum_{n \in \mathbb{N} \backslash X_{m}}\left|m_{n}^{k}\right| \leq M-m \varepsilon
\end{aligned}
$$

para todo $k \in X_{m}$. Se não achamos este conjunto é porque já conseguimos o resultado para um $A_{n}^{j} \subseteq A_{j}$ com $j<m$. Então, seja $\left(A_{i}^{m}\right)_{i \in \omega}$ uma família de subconjuntos infinitos de $X_{m}$ dois a dois disjuntos tais que $X_{m}=\bigcup_{i \in \omega} A_{i}^{m}$. Daí, teremos que ou existe $i \in \omega$ tal que para todo $k \in A_{i}^{m}$ tem-se

$$
\sum_{n \in A_{i}^{m} \backslash\{k\}}\left|m_{n}^{k}\right|<\varepsilon
$$

e temos o resultado, ou acharemos um subconjunto infinito $X_{m+1}$ de $X_{m}$ tal que

$$
\sum_{n \in X_{m+1}}\left|m_{n}^{k}\right| \leq M-(m+1) \varepsilon
$$

para todo $k \in X_{m}$. Assim, seguimos por indução até encontrar $X_{m}$ tal que ou existe $i \in \omega$ tal que $A_{i}^{m}$ satisfaz

$$
\sum_{n \in A_{i}^{m} \backslash\{k\}}\left|m_{n}^{k}\right|<\varepsilon
$$

para todo $k \in A_{i}^{m}$ ou até encontrar $X_{m}$ tal que $m \varepsilon>M$. O processo pára, pois sendo $m \in \omega$ o menor tal que $m \varepsilon>M$, repetindo sucessivamente esta construção, devemos achar um subconjunto que satisfaz as propriedades até o $m$-ésimo passo, pois se não achássemos, teríamos $A_{m} \subseteq \mathbb{N}$ tal que

$$
\sum_{n \in A_{m}}\left|m_{n}^{k}\right| \leq M-m \varepsilon<0
$$

o que não pode ocorrer. 
Assim, suponha que temos $\left(a_{n}\right)_{n \in \mathbb{N}}$ uma seqüência de abertos-fechados em $K$ e $\left(\mu_{k}\right)_{k \in \mathbb{N}}$ uma seqüência de medidas de Radon. Daí, se pudermos garantir que a seqüência de medidas $\left(\mu_{k}\right)_{k \in \mathbb{N}}$ é uniformemente limitada por um $M>0$, então, para cada $k \in \mathbb{N}$, teremos que $\sum_{n \in \mathbb{N}}\left|\mu_{k}\left(a_{n}\right)\right| \leq\left\|\mu_{k}\right\| \leq M$ e portanto, tomando $m_{n}^{k}=\mu_{k}\left(a_{n}\right)$, temos a hipótese deste resultado e podemos aplicá-lo. Isso justifica termos apresentado este resultado aqui.

Apresentamos ainda mais um resultado, que segue do resultado acima, envolvendo seqüências de abertos-fechados que utilizaremos mais à frente:

Lema 3.37. Sejam $K, L$ um espaço booleano e $T: C(K) \rightarrow C(L)$ uma aplicaçấo linear limitada. Suponha que existe uma anticadeia $\left(a_{n}\right)_{n \in \mathbb{N}} \subseteq C l o p(K)$ tal que $\left\|T\left(\chi_{a_{n}}\right)\right\| \nrightarrow 0$ quando $n \rightarrow \infty$. Então existe $M \subseteq \mathbb{N}$ infinito tal que

$$
\left.T\right|_{\overline{\left[a_{n}: n \in M\right]}} \text { é um isomorfismo sobre sua imagem. }
$$

Demonstração. Suponha $\left(a_{n}\right)_{n \in \mathbb{N}} \subseteq C l o p(K)$ como na hipótese. Então, existem $\varepsilon>0$ e $N \subseteq \mathbb{N}$ infinito tais que

$$
\forall n \in N \quad\left\|T\left(\chi_{a_{n}}\right)\right\|>\varepsilon .
$$

Como a norma em $C(L)$ é a norma do supremo, temos que para cada $n \in N$, existe $l_{n} \in \mathbb{N}$ tal que $\left|T\left(\chi_{a_{n}}\right)\left(l_{n}\right)\right|>\varepsilon$. Defina, para todos $k, n \in N, m_{n}^{k}=T\left(\chi_{a_{n}}\right)\left(l_{k}\right)$. Daí, fixado $k \in N$ e $F \subseteq N$ finito, considere $F_{+}=\left\{n \in F: T\left(\chi_{a_{n}}\right)\left(l_{k}\right) \geq 0\right\}$. Temos então que

$$
\begin{aligned}
\sum_{n \in F}\left|m_{n}^{k}\right| & =\sum_{n \in F}\left|T\left(\chi_{a_{n}}\right)\left(l_{k}\right)\right|=\sum_{n \in F_{+}} T\left(\chi_{a_{n}}\right)\left(l_{k}\right)-\sum_{n \in F \backslash F_{+}} T\left(\chi_{a_{n}}\right)\left(l_{k}\right) \\
& =T\left(\sum_{n \in F_{+}} \chi_{a_{n}}-\sum_{n \in F \backslash F_{+}} \chi_{a_{n}}\right)\left(l_{k}\right) \leq\left\|T\left(\sum_{n \in F_{+}} \chi_{a_{n}}-\sum_{n \in F \backslash F_{+}} \chi_{a_{n}}\right)\right\| \\
& \leq\|T\| \cdot\left\|\sum_{n \in F_{+}} \chi_{a_{n}}-\sum_{n \in F \backslash F_{+}} \chi_{a_{n}}\right\|=\|T\| .
\end{aligned}
$$

Portanto, para todo $k \in N, \sum_{n \in N}\left|m_{n}^{k}\right| \leq\|T\|$.

Então, pelo Lema de Rosenthal (3.36), temos que existe $M \subseteq N$ infinito tal que

$$
\forall k \in M \sum_{n \in M \backslash\{k\}}\left|m_{n}^{k}\right|<\frac{\varepsilon}{2} .
$$

Consideremos $M^{\prime} \subseteq M$ finito não vazio e $\left(\alpha_{n}\right)_{n \in M^{\prime}} \subseteq \mathbb{R}$. Tomemos $n_{0} \in M^{\prime}$ tal que $\left|\alpha_{n_{0}}\right|=\max \left\{\left|\alpha_{n}\right|: n \in M^{\prime}\right\}$. Assim, $\left\|\sum_{n \in M^{\prime}} \alpha_{n} \chi_{a_{n}}\right\|=\left|\alpha_{n_{0}}\right|$, uma vez que $\left(a_{n}\right)_{n \in \mathbb{N}}$ é uma anticadeia. Assim,

$$
\left\|T\left(\sum_{n \in M^{\prime}} \alpha_{n} \chi_{a_{n}}\right)\right\| \leq\|T\| \cdot\left|\alpha_{n_{0}}\right|
$$


e por outro lado, temos

$$
\left\|T\left(\sum_{n \in M^{\prime}} \alpha_{n} \chi_{a_{n}}\right)\right\|=\left\|\sum_{n \in M^{\prime}} \alpha_{n} T\left(\chi_{a_{n}}\right)\right\| \geq\left|\sum_{n \in M^{\prime}} \alpha_{n} T\left(\chi_{a_{n}}\right)\left(l_{n_{0}}\right)\right| \geq\left|\alpha_{n_{0}}\right| \cdot \varepsilon-\left|\alpha_{n_{0}}\right| \cdot \frac{\varepsilon}{2}=\left|\alpha_{n_{0}}\right| \cdot \frac{\varepsilon}{2}
$$

Logo, para qualquer $M^{\prime} \subseteq M$ finito e $\left(\alpha_{n}\right)_{n \in M^{\prime}} \subseteq \mathbb{R}$ temos que

$$
\frac{\varepsilon}{2} \cdot\left\|\sum_{n \in M^{\prime}} \alpha_{n} \chi_{a_{n}}\right\| \leq\left\|T\left(\sum_{n \in M^{\prime}} \alpha_{n} \chi_{a_{n}}\right)\right\| \leq\|T\| \cdot\left\|\sum_{n \in M^{\prime}} \alpha_{n} \chi_{a_{n}}\right\| .
$$

Então, para todo $f \in \overline{\left[\chi_{a_{n}}: n \in M\right]}$, temos que

$$
\frac{\varepsilon}{2} \cdot\|f\| \leq\|T(f)\| \leq\|T\| \cdot\|f\| .
$$

Portanto, $\left.T\right|_{\overline{\left[\chi_{n}: n \in M\right]}}$ é um isomorfismo sobre sua imagem.

\subsection{Operadores fracamente compactos em $C(K)$}

Nosso primeiro objetivo nesta seção é verificar que espaços da forma $C(K)$, onde $K$ é um espaço booleano, têm a propriedade de Dunford-Pettis:

Definição 3.38. Seja $X$ um espaço de Banach. Dizemos que $X$ tem a propriedade de Dunford-Pettis ${ }^{11}$ se para toda seqüência $\left(\Phi_{n}\right)_{n \in \mathbb{N}} \subseteq X^{*}$ fracamente nula e toda seqüência $\left(x_{n}\right)_{n \in \mathbb{N}} \subseteq X$ fracamente nula tem-se que a seqüência $\left(\Phi_{n}\left(x_{n}\right)\right)_{n \in \mathbb{N}}$ converge a 0 .

Teorema 3.39. Se K é um espaço booleano, então $C(K)$ tem a propriedade de DunfordPettis.

Demonstração. Sejam $\left(\mu_{n}\right)_{n \in \mathbb{N}} \subseteq C(K)^{*}$ e $\left(f_{n}\right)_{n \in \mathbb{N}} \subseteq C(K)$ seqüências fracamente nulas. Temos então que para todo $x \in K,\left(f_{n}(x)\right)_{n \in \mathbb{N}}$ converge a 0 . Como $\left(\mu_{n}\right)_{n \in \mathbb{N}}$ converge fracamente a 0 , temos, pelo Corolário 3.15 que $\left\{\mu_{n}(f): f \in C(K)\right\}$ é limitado. Daí, pelo Princípio de Limitação Uniforme (2.6), temos que $\left\{\mu_{n}: n \in \mathbb{N}\right\}$ é limitado. Seja $M>0$ tal que $\left\|\mu_{n}\right\| \leq M$ para todo $n \in \mathbb{N}$.

Dado $\delta>0$ e $n \in \mathbb{N}$, defina

$$
E_{n}=\left\{x \in K:\left|f_{n}(x)\right| \geq \delta\right\} .
$$

\footnotetext{
${ }^{11}$ Esta propriedade é usualmente definida de outra forma. Nossa definição é a de [Di] e equivale à usual.
} 
Temos que cada $x \in K$ pertence apenas a um número finito de tais $E_{n}$ 's. Logo,

$$
\bigcap_{m \in \mathbb{N} n \geq m} \bigcup_{n} E_{n}=\left\{x \in K: \forall m \exists n \geq m x \in E_{n}\right\}=\emptyset .
$$

Dado então $\delta^{\prime}>0$, suponhamos por absurdo que existem infinitos $k$ 's tais que

$$
\left|\mu_{k}\right|\left(\bigcup_{n \geq k} E_{n}\right)>\delta^{\prime}
$$

Então, podemos obter $\left(k_{m}\right)_{m \in \mathbb{N}} \subseteq \mathbb{N}$ crescente tal que

$$
\forall m \in \mathbb{N} \quad\left|\mu_{k_{m}}\right|\left(\bigcup_{n=k_{m}+1}^{k_{m+1}} E_{n} \backslash E_{n+1}\right)>\delta^{\prime} .
$$

Pela regularidade de cada $\mu_{k_{m}}$ e usando que $K$ é booleano, podemos obter $\left(a_{m}\right)_{m \in \mathbb{N}} \subseteq$ $C l o p(K)$ dois a dois disjuntos tais que

$$
\forall m \in \mathbb{N} \quad\left|\mu_{k_{m}}\right|\left(a_{m}\right)>\delta
$$

Fixemos $i \in \mathbb{N}$. Temos que, para todo $l \in \mathbb{N}$,

$$
\sum_{n=0}^{l}\left|\mu_{k_{i}}\right|\left(a_{n}\right)=\left|\mu_{k_{i}}\right|\left(\bigcup_{n=0}^{l} a_{n}\right) \leq\left\|\mu_{k_{i}}\right\| \leq M .
$$

Logo, temos que $\sum_{n=0}^{\infty}\left|\mu_{k_{i}}\right|\left(a_{n}\right) \leq M$ para todo $i \in \mathbb{N}$. Daí, pelo Lema de Rosenthal (3.36), existe $L \subseteq \mathbb{N}$ infinito tal que para todo $i \in L$,

$$
\sum_{n \in L \backslash\{i\}}\left|\mu_{k_{i}}\right|\left(a_{n}\right)<\frac{\delta}{2}
$$

Seja $a=\bigcup_{n \in L} a_{n} \in \operatorname{Bor}(K)$. Temos que para todo $i \in L$,

$$
\left|\mu_{k_{i}}(a)\right| \geq\left|\mu_{k_{i}}\left(a_{i}\right)\right|-\sum_{n \in L \backslash\{i\}}\left|\mu_{k_{i}}\right|\left(a_{n}\right) \geq \varepsilon-\frac{\varepsilon}{2}=\frac{\varepsilon}{2} .
$$

Portanto, pelo Lema 3.16, $\left(\mu_{k}\right)_{k \in \mathbb{N}}$ não converge fracamente a 0 , uma contradição.

Assim, para todo $\delta^{\prime}>0$, existe $k_{0} \in \mathbb{N}$ tal que se $k \geq k_{0}$, então $\left|\mu_{k}\right|\left(\bigcup_{n \geq k} E_{n}\right) \leq \delta^{\prime}$. Logo, se $k \geq k_{0}$, temos

$$
\mu_{k}\left(f_{k}\right)=\int_{K} f_{k} d \mu_{k}=\int_{E_{k}} f_{k} d \mu_{k}+\int_{K \backslash E_{k}} f_{k} d \mu_{k} \leq \delta^{\prime}\left\|f_{k}\right\|+\delta\left\|\mu_{k}\right\| .
$$

Daí, dado $\varepsilon>0$, escolhendo $\delta, \delta^{\prime}>0$ tais que $\delta^{\prime}\left\|f_{k}\right\|+\delta\left\|\mu_{k}\right\|<\varepsilon$, temos que se $k \geq k_{0}$, então $\mu_{k}\left(f_{k}\right)<\varepsilon$. Portanto, $\left(\mu_{k}\left(f_{k}\right)\right)_{k \in \mathbb{N}}$ converge a 0 . 
Vejamos que em espaços $C(K)$ os operadores fracamente compactos são caracterizados por outras propriedades muito interessantes. Primeiramente, uma definição:

Definição 3.40. Sejam $X, Y$ espaços de Banach. Dizemos que uma aplicação linear limitada $T: X \rightarrow Y$ é completamente contínua se leva seqüências fracamente nulas de $X$ em seqüências de $Y$ convergentes a zero (na norma).

Proposição 3.41. Sejam $K, L$ espaços booleanos e $T: C(K) \rightarrow C(L)$ uma aplicação linear limitada. São equivalentes:

(a) Té fracamente compacta;

(b) Té completamente contínua;

(c) não existe $X$ uma cópia de $c_{0}$ em $C(K)$ tal que $\left.T\right|_{X}$ é um isomorfismo sobre sua imagem;

(d) para toda anticadeia $\left(a_{n}\right)_{n \in \mathbb{N}} \subseteq C l o p(K)$ tem-se que $\left(T\left(\chi_{a_{n}}\right)\right)_{n \in \mathbb{N}}$ converge a 0 (na norma).

Demonstração. (a) $\Rightarrow$ (b)

Suponhamos $T$ fracamente compacta e suponhamos, por absurdo, que existe $\left(f_{n}\right)_{n \in \mathbb{N}} \subseteq$ $C(K)$ uma seqüência fracamente nula tal que $\left(T\left(f_{n}\right)\right)_{n \in \mathbb{N}}$ não converge a 0 . Daí, existem $\varepsilon>0$ e $\left(n_{k}\right)_{k \in \mathbb{N}} \subseteq \mathbb{N}$ crescente tais que para todo $k \in \mathbb{N}$, tem-se que $\left\|T\left(f_{n_{k}}\right)\right\| \geq \varepsilon$. Logo, para cada $k \in \mathbb{N}$, existe $x_{k} \in K$ tal que $\left|T\left(f_{n_{k}}\right)\left(x_{k}\right)\right| \geq \varepsilon$.

Considere $\delta_{x_{k}} \in C(K)^{*}$ tal que $\delta_{x_{k}}(f)=f\left(x_{k}\right)$. Temos que $\left\{\delta_{x_{k}}: k \in \mathbb{N}\right\} \subseteq C(K)^{*}$ é limitado. Como, pela proposição anterior, $T^{*}$ é fracamente compacta, segue que o conjunto $\left\{T^{*}\left(\delta_{x_{k}}\right): k \in \mathbb{N}\right\}$ é relativamente fracamente compacto. Logo, pelo Teorema de Eberlein-ŠSmulian (2.15), existem $\left(k_{i}\right)_{i \in \mathbb{N}} \subseteq \mathbb{N}$ crescente e $\mu \in C(K)^{*}$ tais que $\left(T^{*}\left(\delta_{x_{k_{i}}}\right)\right)_{i \in \mathbb{N}}$ é fracamente convergente a $\mu$ e, portanto, $\left(T^{*}\left(\delta_{x_{k_{i}}}\right)-\mu\right)_{i \in \mathbb{N}}$ é fracamente nula.

Segue, da propriedade de Dunford-Pettis, que $\left(T^{*}\left(\delta_{x_{k_{i}}}\right)\left(f_{n_{k_{i}}}\right)-\mu\left(f_{n_{k_{i}}}\right)\right)_{i \in \mathbb{N}}$ converge a zero. Por outro lado, como $\left(f_{n_{k_{i}}}\right)_{i \in \mathbb{N}}$ converge fracamente a 0 , temos que $\left(\mu\left(f_{n_{k_{i}}}\right)\right)_{i \in \mathbb{N}}$ converge a 0 . Logo, $\left(T^{*}\left(\delta_{x_{k_{i}}}\right)\left(f_{n_{k_{i}}}\right)\right)_{i \in \mathbb{N}}$ converge a 0 .

Mas para todo $k \in \mathbb{N}$, temos que $\left|T^{*}\left(\delta_{x_{k}}\right)\left(f_{n_{k}}\right)\right|=\left|T\left(f_{n_{k}}\right)\left(x_{k}\right)\right| \geq \varepsilon$, uma contradição. Portanto, $T$ é completamente contínua. 
(b) $\Rightarrow(\mathbf{c})$

Suponhamos $T$ completamente contínua e $X \subseteq C(K)$ uma cópia de $c_{0}$. Considere $S: c_{0} \rightarrow X$ um isomorfismo e seja, para cada $n \in \mathbb{N}, f_{n}=S\left(e_{n}\right)$.

Dada $\Phi=\left(\alpha_{n}\right)_{n \in \mathbb{N}} \in l_{1}=c_{0}^{*}$, temos que $\Phi\left(e_{n}\right)=\alpha_{n}$ e daí, como $\Phi \in l_{1},\left(\Phi\left(e_{n}\right)\right)_{n \in \mathbb{N}}$ converge a 0 . Logo, $\left(e_{n}\right)_{n \in \mathbb{N}}$ converge fracamente a 0 e, como $S$ é um isomorfismo, temos que $\left(f_{n}\right)_{n \in \mathbb{N}}$ converge fracamente a $0 \mathrm{em} X$.

Seja $\mu \in C(K)^{*}$. Temos que $\left.\mu\right|_{X} \in X^{*}$. Logo, $\left(\mu\left(f_{n}\right)\right)_{n \in \mathbb{N}}=\left(\left.\mu\right|_{X}\left(f_{n}\right)\right)_{n \in \mathbb{N}}$ converge a 0 . Assim, $\left(f_{n}\right)_{n \in \mathbb{N}}$ converge fracamente a 0 em $C(K)$. Como $T$ é completamente contínua, $T\left(f_{n}\right)_{n \in \mathbb{N}}$ converge a 0 e, portanto, $\left.T\right|_{X}$ não é um isomorfismo sobre sua imagem.

(c) $\Rightarrow(d)$

Suponhamos que existe uma anticadeia $\left(a_{n}\right)_{n \in \mathbb{N}} \subseteq C \operatorname{lop}(K)$ tal que $\left(T\left(\chi_{a_{n}}\right)\right)_{n \in \mathbb{N}}$ não converge a 0 . Daí, pelo Lema 3.37 , existe $M \subseteq \mathbb{N}$ infinito tal que $\left.T\right|_{\overline{\left[\chi_{a_{n}}: n \in M\right]}}$ é um isomorfismo sobre sua imagem. Vejamos que $X=\overline{\left[\chi_{a_{n}}: n \in M\right]}$ é isométrico a $c_{0}$.

Fixemos $M=\left\{m_{n}: n \in \mathbb{N}\right\}$ uma enumeração para $M$. Seja $Q: X \rightarrow c_{0}$ aplicação linear dada por $Q\left(\chi_{a_{m_{n}}}\right)=e_{n}$. Assim, temos $Q$ definida para todo elemento de $\left[\chi_{a_{n}}: n \in\right.$ $M]$ e, como este conjunto é denso em $X, Q$ está bem definida para todo elemento de $X$. $Q$ é linear, por definição e $Q$ é sobrejetora, pois $\left[e_{n}: n \in \mathbb{N}\right] \subseteq \operatorname{Im}(Q)$ e $\left[e_{n}: n \in \mathbb{N}\right]$ é denso em $c_{0}$.

Temos que

$$
\left\|Q\left(\chi_{a_{n}}\right)\right\|=\left\|e_{n}\right\|=1=\left\|\chi_{a_{n}}\right\|
$$

Logo, dados $M^{\prime} \subseteq \mathbb{N}$ finito e $\left(\alpha_{n}\right)_{n \in M^{\prime}} \subseteq \mathbb{R}$, seja $n_{0} \in M^{\prime}$ tal que $\left|\alpha_{n_{0}}\right|=\max _{n \in M^{\prime}}\left\{\left|\alpha_{n}\right|\right.$ : $\left.n \in M^{\prime}\right\}$ e temos

$$
\left\|Q\left(\sum_{n \in M^{\prime}} \alpha_{m_{n}} \chi_{a_{n}}\right)\right\|=\left\|\sum_{n \in M^{\prime}} \alpha_{m_{n}} Q\left(\chi_{a_{n}}\right)\right\|=\left\|\sum_{n \in M^{\prime}} \alpha_{n} e_{n}\right\|=\left|\alpha_{n_{0}}\right|=\left\|\sum_{n \in M^{\prime}} \alpha_{n} \chi_{a_{m_{n}}}\right\| .
$$

Assim, temos que $Q$ é injetora e preserva a norma. Portanto, $Q$ é uma isometria de $X$ sobre $c_{0}$.

(d) $\Rightarrow($ b)

Se $T$ não é fracamente compacto, pelo Teorema de Gantmacher (2.24), temos que $T^{*}: C(K)^{*} \rightarrow C(K)^{*}$ não é fracamente compacto. Então existe $X \subseteq C(K)^{*}$ limitado tal que $T^{*}[X]$ não é relativamente fracamente compacto. 
Seja $M>0$ tal que $\|\mu\| \leq M$ para todo $\mu \in X$. Temos então que, para todo $\mu \in X$,

$$
\left\|T^{*}(\mu)\right\| \leq\left\|T^{*}\right\| \cdot\|\mu\| \leq\left\|T^{*}\right\| \cdot M
$$

Assim, $T^{*}[X]$ é limitado e não é relativamente fracamente compacto. Logo, pelo Teorema de Dieudonné-Grothendieck (3.17), existe uma seqüência de abertos $\left(U_{n}\right)_{n \in \mathbb{N}}$ dois a dois disjuntos tais que $\left(T^{*}(\mu)\left(U_{n}\right)\right)_{n \in \mathbb{N}}$ não converge uniformemente a 0 para $\mu \in X$. Como $K$ é booleano, podemos supor, sem perda de generalidade que $U_{n}=a_{n}$, onde $\left(a_{n}\right)_{n \in \mathbb{N}} \subseteq$ Clop $(K)$.

Suponhamos, por absurdo, que $\left\|T\left(a_{n}\right)\right\|$ converge a 0 . Daí, dado $\varepsilon>0$, existe $n_{0} \in \mathbb{N}$ tal que se $n \geq n_{0}$, então $\left\|T\left(a_{n}\right)\right\| \leq \frac{\varepsilon}{M}$. Segue que, para todo $\mu \in X$,

$$
\left|T^{*}(\mu)\left(a_{n}\right)\right|=\left|\mu\left(T\left(a_{n}\right)\right)\right| \leq\|\mu\| \cdot\left\|T\left(a_{n}\right)\right\|<M \cdot \frac{\varepsilon}{M}=\varepsilon,
$$

contradizendo que $\left(T^{*}(\mu)\left(U_{n}\right)\right)_{n \in \mathbb{N}}$ não converge uniformemente a 0 para $\mu \in X$.

Portanto, $\left\|T\left(a_{n}\right)\right\|$ não converge a 0.

(b) $\Rightarrow$ (a)

Suponhamos por absurdo, que $T$ é completamente contínua e não é fracamente compacta. Daí, pela Proposição 2.24, $T^{*}$ não é fracamente compacta. Logo, existe $M \subseteq C(K)^{*}$ limitado tal que $T^{*}[M]$ não é relativamente fracamente compacto. Mas se $M$ é limitado, então $T^{*}[M]$ também é limitado. Logo, pelo Teorema de Dieudonné-Grothendieck (3.17), existe $\left(a_{n}\right)_{n \in \mathbb{N}}$ abertos dois a dois disjuntos tais que $\left(T^{*}(\mu)\left(a_{n}\right)\right)_{n \in \mathbb{N}}$ não converge uniformemente a 0 para $\mu \in M$. Como $K$ é booleano $T^{*}[M]$ é um conjunto de medidas de Radon, e portanto, regulares, podemos supor, sem perda de generalidade, que $\left(a_{n}\right)_{n \in \mathbb{N}} \subseteq$ Clop $(K)$.

Daí, existem $\varepsilon>0,\left(n_{k}\right)_{k \in \mathbb{N}} \subseteq \mathbb{N}$ crescente e $\left(\mu_{k}\right)_{k \in \mathbb{N}} \subseteq M$ tais que

$$
\forall k \in \mathbb{N} \quad\left|\mu_{k}\left(T\left(\chi_{a_{n_{k}}}\right)\right)\right|=\left|T^{*}\left(\mu_{k}\right)\left(a_{n_{k}}\right)\right| \geq \varepsilon
$$

Por outro lado, é fácil ver que $\left(\chi_{a_{n}}\right)_{n \in \mathbb{N}} \subseteq C(K)$ converge fracamente a 0 . Então, como $T$ é completamente contínua, temos que $\left(T\left(\chi_{a_{n}}\right)\right)_{n \in \mathbb{N}} \subseteq C(K)$ converge a 0 . Além disso, como $M$ é limitado, existe $M>0$ tal que para todo $k \in \mathbb{N}$, $\left\|\mu_{k}\right\| \leq M$. Logo,

$$
\left|\mu_{k}\left(T\left(\chi_{a_{n_{k}}}\right)\right)\right| \leq\left\|\mu_{k}\right\| \cdot\left\|T\left(\chi_{a_{n_{k}}}\right)\right\| \leq M \cdot\left\|T\left(\chi_{a_{n_{k}}}\right)\right\| \longrightarrow 0
$$

contradizendo $(*)$. 
Por fim, um resultado que ajuda no estudo dos possíveis subespaços complementados de um espaço da forma $C(K)$ :

Proposição 3.42. Seja $K$ um espaço booleano. Se $X$ é um subespaço de $C(K)$ de dimensão infinita e $P: C(K) \rightarrow X$ é uma projeção de $C(K)$ sobre $X$, então $P$ não é fracamente compacta.

Demonstração. Suponhamos, por absurdo, que $P: C(K) \rightarrow X$ é uma projeção sobre $X$, que $P$ é fracamente compacta e que $X$ tem dimensão infinita. $O$ fato que $X$ tem dimensão infinita implica, pelo Teorema 2.2, que a bola unitária de $X, B_{X}$ não é compacta e, portanto, não é seqüencialmente compacta. Daí, existe $\left(f_{n}\right)_{n \in \mathbb{N}} \subseteq B_{X}$ sem subseqüências convergentes.

Como $B_{X}$ é limitado e $P$ é fracamente compacto, temos que $\left\{P\left(f_{n}\right): n \in \mathbb{N}\right\}=\left\{f_{n}\right.$ : $n \in \mathbb{N}\}$ é relativamente fracamente compacto. Pelo Teorema de Eberlein-Šmulian (2.15), existe $\left(n_{k}\right)_{k \in \mathbb{N}} \subseteq \mathbb{N}$ crescente e $f \in X$ tal que $\left(f_{n_{k}}\right)_{k \in \mathbb{N}}$ é fracamente convergente a $f$. Logo, $\left(f_{n_{k}}-f\right)_{k \in \mathbb{N}}$ é fracamente nula. Pela proposição anterior, temos que se $P$ é fracamente compacta, então $P$ é completamente contínua. Assim, $\left(P\left(f_{n_{k}}-f\right)\right)_{k \in \mathbb{N}}=\left(f_{n_{k}}-f\right)_{k \in \mathbb{N}}$ converge a 0 e, portanto, $\left(f_{n_{k}}\right)_{k \in \mathbb{N}}$ converge a $f$, contradizendo o fato que $\left(f_{n}\right)_{n \in \mathbb{N}}$ não tem subseqüências convergentes.

Por fim, um resultado importante devido a Pełczyński:

Corolário 3.43. Seja $K$ um espaço booleano e $X$ um subespaço complementado em $C(K)$ de dimensão infinita. Então $X$ tem uma cópia de $c_{0}$.

Demonstração. Seja $P: C(K) \rightarrow X$ uma projeção. Pela proposição anterior, temos que $P$ não é fracamente compacta. Logo, pela Proposição 3.41 , temos que existe em $C(K)$ uma cópia $Y$ de $c_{0}$ tal que $\left.P\right|_{Y}$ é um isomorfismo sobre sua imagem. Assim, $P[Y]$ é uma cópia de $c_{0}$ em $X$.

\subsection{A propriedade de Grothendieck em $C(K)$}

Analisamos, por fim, como se comporta a propriedade de Grothendieck em espaços da forma $C(K)$ e um exemplo de tal espaço. 


\subsubsection{Uma caracterização}

Em 1982, Schachermayer provou em seu trabalho (veja [Scha]), a seguinte caracterização para espaços de Grothendieck.

Teorema 3.44. Seja K um espaço booleano. São equivalentes as seguintes afirmações:

(a) $C(K)$ é de Grothendieck;

(b) $C(K)$ não possui cópias complementadas de $c_{0}$.

Demonstração. (a) $\Rightarrow(\mathrm{b})$. Suponhamos, por absurdo, que $C(K)$ é Grothendieck e que $C(K)$ possui uma cópia complementada de $c_{0}$. Então existe $X \subseteq C(K) \operatorname{com} X \sim c_{0} \mathrm{e}$ $P: C(K) \rightarrow X$ uma projeção. Daí, pela Proposição 2.42, temos que $X$ é Grothendieck e, portanto, $c_{0}$ é Grothendieck. Porém, pelo Exemplo 2.41, $c_{0}$ não é Grothendieck. Logo, se $C(K)$ é Grothendieck, então $C(K)$ não possui uma cópia complementada de $c_{0}$.

(b) $\Rightarrow(\mathrm{a})$. Suponhamos que $C(K)$ não é Grothendieck. Daí, existe uma seqüência de medidas de Radon $\left(\mu_{n}\right)_{n \in \mathbb{N}} \subseteq C(K)^{*}$ que converge na topologia fraca* a $\mu \in C(K)^{*}$ e não converge na topologia fraca. Pelo Lema 3.19, temos que existem uma anticadeia $\left(a_{n}\right)_{n \in \mathbb{N}} \subseteq C \operatorname{lop}(K)$ e $\left(k_{n}\right)_{n \in \mathbb{N}} \subseteq \mathbb{N}$ crescente tais que

$$
\forall n \in \mathbb{N} \quad\left|\mu_{k_{n}}\left(a_{n}\right)\right| \geq \varepsilon
$$

Definimos então $T: C(K) \rightarrow c_{0}$ por $T(f)=\left(\mu_{k_{n}}(f)-\mu(f)\right)_{n \in \mathbb{N}}$. $T$ está bem definida, pois $\left(\mu_{n}\right)_{n \in \mathbb{N}}$ converge na topologia fraca* a $\mu$. É fácil ver que $T$ é linear. $T$ é limitada, pois dada $f \in C(K)$, temos

$$
\|T(f)\|=\left\|\left(\mu_{k_{n}}(f)-\mu(f)\right)_{n \in \mathbb{N}}\right\|=\sup _{n \in \mathbb{N}}\left|\mu_{k_{n}}(f)-\mu(f)\right| \leq 2\|\mu\| \cdot\|f\| .
$$

Temos ainda que existe $L \subseteq \mathbb{N}$ infinito tal que para todo $n \in L$, tem-se que $|\mu|\left(a_{n}\right) \leq \frac{\varepsilon}{2}$, pois senão, existiria $L \subseteq \mathbb{N}$ infinito tal que para todo $n \in L,|\mu|\left(a_{n}\right)>\frac{\varepsilon}{2}$ e daí,

$$
|\mu|\left(\bigcup_{n \in L} a_{n}\right) \geq \sum_{n \in L}|\mu|\left(a_{n}\right)=\infty
$$

contradizendo que $\mu$ é limitada. 
Assim, temos tal $L$ e, para todo $n \in L$,

$$
\left\|T\left(\chi_{a_{n}}\right)\right\|=\left\|\mu_{k_{n}}\left(a_{n}\right)-\mu\left(a_{n}\right)\right\| \geq\left|\mu_{k_{n}}\left(a_{n}\right)\right|-|\mu|\left(a_{n}\right) \geq \varepsilon-\frac{\varepsilon}{2}=\frac{\varepsilon}{2} .
$$

Logo, pelo Lema 3.37, existe $M \subseteq L$ infinito tal que $\left.T\right|_{\overline{\left[{\chi_{n}}_{n}: n \in M\right]}}$ é um isomorfismo sobre sua imagem. Seja $X=\overline{\left[\chi_{a_{n}}: n \in M\right]}$ e como na demonstração da Proposição 3.41 temos que $X \equiv c_{0}$.

Tomemos $Y=T[X] \sim c_{0}$. Pelo Teorema de Sobczyk (3.25), existe uma projeção $P$ de $c_{0}$ sobre $Y$, uma vez que $c_{0}$ é separável e $Y \sim c_{0}$. Seja ainda $S: Y \rightarrow X$ a inversa de $\left.T\right|_{X}$. Defina enfim $P^{\prime}=S \circ P \circ T: C(K) \rightarrow X$. É claro que $P^{\prime}$ está bem definida. Vejamos que é uma projeção de $C(K)$ sobre $X$. $P^{\prime}$ é linear e limitada, pois $S, P$ e $T$ o são.

Além disso, se $n \in M$, como $T\left(\chi_{a_{n}}\right) \in Y$ e $\left.P\right|_{Y}=I d$, temos que

$$
P^{\prime}\left(\chi_{a_{n}}\right)=S \circ P \circ T\left(\chi_{a_{n}}\right)=S \circ T\left(\chi_{a_{n}}\right)=\chi_{a_{n}} .
$$

Logo, para todo $f \in\left[\chi_{a_{n}}: n \in M\right]$ temos que $P^{\prime}(f)=f$. Portanto, $\left(P^{\prime}\right)^{2}=P^{\prime}$ e $P^{\prime}[C(K)]=X$, uma vez que $\left[\chi_{a_{n}}: n \in M\right] \subseteq P^{\prime}[C(K)]$ e é denso em $X$.

Utilizaremos tal caracterização para mostrar que alguns dos espaços dos Capítulos 5, 6 e 7 possuem a propriedade de Grothendieck.

Corolário 3.45. Seja $K$ um espaço booleano. Se $C(K)$ é injetivo, então $C(K)$ é Grothen$\operatorname{dieck}^{12}$.

Demonstração. Suponhamos, por absurdo, que $C(K)$ é injetivo e não é Grothendieck. Pelo teorema anterior, temos que existe $P: C(K) \rightarrow c_{0}$ uma projeção. Daí, temos que $c_{0}$ é injetivo, pois dados $X, Y$ espaços de Banach, tais que $Y$ é subespaço de $X$ e $T: Y \rightarrow c_{0} \subseteq C(K)$ linear e limitada, como $C(K)$ é injetivo, existe $\tilde{T}: X \rightarrow C(K)$ linear e limitada que estende $T$. Daí, $P \circ \tilde{T}: X \rightarrow c_{0}$ é linear e limitada e estende $T$.

Por outro lado, pelo Lema de Philips (Proposição 3.26), temos que $c_{0}$ não é injetivo. Portanto, $C(K)$ deve ser Grothendieck.

\footnotetext{
${ }^{12} \mathrm{Na}$ realidade, todo espaço de Banach injetivo tem a propriedade de Grothendieck. Veja [Ma], Teorema 9.4 , página 33 .
} 


\subsubsection{A propriedade de completude subseqüencial}

Nosso objetivo aqui é apresentar um primeiro exemplo não trivial de espaço da forma $C(K)$ com a propriedade de Grothendieck.

Definição 3.46. Seja A uma álgebra de Boole. Dizemos que A tem a propriedade de completude subseqüencial, ou simplesmente, A tem PCS, se para toda anticadeia $\left(a_{n}\right)_{n \in \mathbb{N}} \subseteq A$, existe $M \subseteq \mathbb{N}$ infinito tal que $\sum_{n \in M} a_{n} \in A$.

Teorema 3.47. Seja A uma álgebra de Boole e $K$ seu espaço de Stone. Se A tem a propriedade de completude subseqüencial, então $C(K)$ tem a propriedade de Grothendieck.

Demonstração. Pelo Teorema de Representação de Riesz, temos que $C(K)^{*}$ é isométrico ao espaço de medidas de Radon sobre $K$. Suponha, por absurdo, que $\left(\mu_{n}\right)_{n \in \omega}$ é uma seqüência de medidas de Radon que não converge fracamente mas que é convergente a uma medida de Radon $\mu$ na topologia fraca*.

Pelo Lema 3.19 , temos que existe $\varepsilon>0,\left(a_{n}\right)_{n \in \mathbb{N}} \subseteq A$ anticadeia e $\left(k_{n}\right)_{n \in \mathbb{N}} \subseteq \mathbb{N}$ crescente tais que

$$
\forall n \in \mathbb{N} \quad\left|\mu_{k_{n}}\left(a_{n}\right)\right| \geq \varepsilon
$$

Pelo Lema de Rosenthal (3.36), existe $L \subseteq \omega$ infinito tal que $\sum_{n \in L \backslash\{k\}}\left|\mu_{k}\right|\left(a_{n}\right)<\frac{\varepsilon}{3}$ para todo $k \in L$. Pelo Lema 3.35 , podemos assumir que $\mu_{k}(a)=\sum_{n \in M} \mu_{k}\left(a_{n}\right)$, para cada $k \in L$ e $M \subseteq L$. Mas, pela propriedade de completude subseqüencial, existe $M \subseteq L$ infinito e com $L \backslash M$ infinito tal que $a=\sum_{n \in M} a_{n} \in A$.

Vejamos que $a$ separa $\left(\mu_{k}\right)_{k \in L \backslash M}$ de $\left(\mu_{k}\right)_{k \in M}$ na topologia fraca*. Se $k \in L \backslash M$, temos que

$$
\left|\mu_{k}(a)\right|=\left|\sum_{n \in M} \mu_{k}\left(a_{n}\right)\right| \leq \sum_{n \in M}\left|\mu_{k}\left(a_{n}\right)\right| \leq \sum_{n \in M}\left|\mu_{k}\right|\left(a_{n}\right) \leq \sum_{n \in L \backslash\{k\}}\left|\mu_{k}\right|\left(a_{n}\right)<\frac{\varepsilon}{3} .
$$

Se $k \in M$, temos que

$$
\left|\mu_{k}\left(\sum_{n \in M \backslash\{k\}} a_{n}\right)\right|=\left|\sum_{n \in M \backslash\{k\}} \mu_{k}\left(a_{n}\right)\right| \leq \sum_{n \in M \backslash\{k\}}\left|\mu_{k}\right|\left(a_{n}\right) \leq \sum_{n \in L \backslash\{k\}}\left|\mu_{k}\right|\left(a_{n}\right)<\frac{\varepsilon}{3}
$$

e então,

$$
\left|\mu_{k}(a)\right|=\left|\mu_{k}\left(a_{k}\right)+\sum_{n \in M \backslash\{k\}} \mu_{k}\left(a_{n}\right)\right| \geq \frac{2 \varepsilon}{3} .
$$


Portanto, pelo Lema 3.18 , temos que $\left(\mu_{n}\right)_{n \in \omega}$ não é convergente na topologia fraca*, contradizendo nossa hipótese.

Corolário 3.48. Seja A uma álgebra de Boole e $K$ seu espaço de Stone. Se A é $\sigma$ completa, então $C(K)$ tem a propriedade de Grothendieck.

Demonstração. Basta notar que se $A$ é $\sigma$-completa, então $A$ tem PCS.

Temos agora um painel geral sobre espaços de Grothendieck e pretendemos então estudar exemplos de tais espaços que possuem outras propriedades interessantes. 


\section{Capítulo 4}

\section{O método de forcing}

O objetivo deste capítulo é introduzir o método de forcing ${ }^{1}$, com os principais resultados da teoria, e apresentar alguns teoremas sobre forcing's com certas boas propriedades. Na primeira seção, temos o primeiro resultado importante deste capítulo, que garante a força deste método:

Teorema (Princípio de Forcing). Para mostrar que uma sentença $\Phi$ é consistente ${ }^{2}$, basta mostrar que para todo modelo transitivo e enumerável $M$ de $Z F C^{B}$, existe um modelo

\footnotetext{
${ }^{1}$ O forcing é um método criado por Paul Cohen em 1963-64, pelo qual ele recebeu a medalha Fields em 1964 ([Co1] e [Co2]). Mais especificamente, usando este método ele mostrou que o problema do contínuo de Cantor, primeiro da lista de Hilbert do congresso de Paris de 1900, é indecidível (Veja [Go]). O método pertence formalmente à lógica e é usado para mostrar que, dada uma afirmação matemática, nem ela nem sua negação tem uma demonstração. Ou seja, ele serve para mostrar que o problema de que se esta afirmação é verdadeira é indecidível ou, em outras palavras, insolúvel usando as ferramentas usuais da matemática. Na forma moderna, desenvolvida no começo dos anos setenta, este é um método combinatório que trata de estruturas onde os axiomas da teoria dos conjuntos são verdadeiros (modelos de ZFC). Desde Cohen, numerosos problemas foram mostrados indecidíveis, usando o método de forcing. Alguns exemplos são a Conjectura de Borel sobre conjuntos de medida nula forte (veja [La]), a Conjectura de Kaplansky sobre álgebras de Banach (veja [DW]) e a Conjectura de Whitehead sobre o funtor Ext para grupos abelianos (veja [Ek1] e [Ek2].

${ }^{2}$ Dizemos que uma sentença é consistente se assumindo que os axiomas de ZFC são consistentes, temos que a teoria obtida adicionando tal sentença a ZFC também é consistente. Isto é equivalente ao fato que a negação da sentença não pode ser provada de ZFC, a menos que ZFC tenha contradições. Na realidade, esta é a definição de ser relativamente consistente, mas usamos consistente, por abuso de linguagem. Veja $[\mathrm{Ku}]$ para mais detalhes.

${ }^{3}$ Chamamos de ZFC o conjunto dos axiomas conhecidos como axiomas de Zermelo Fraenkel com o
} 
transitivo e enumerável $N$ tal que $M \subseteq N$ e $N \vDash Z F C+\Phi$.

O forcing é uma técnica que, dados um modelo transitivo e enumerável $(M)$ para ZFC e uma ordem parcial $(\mathbb{P})$, nos fornece um modelo transitivo e enumerável (que denotaremos por $M[G]$ ), que contém o inicial, e em que as afirmações são, de certa forma, controladas pela ordem parcial. Assim, pelo Princípio de Forcing, temos uma forte ferramenta para provar a consistência ou a independência ${ }^{4}$ de afirmações.

Na Seção 3.2, cuidamos de definir o modelo $M[G]$ e mostrar que ele é transitivo, enumerável, satisfaz ZFC, e diversas outras propriedades que serão necessárias posteriormente. Aqui, temos o seguinte resultado:

Teorema. $M[G]$ satisfaz $Z F C$.

Abordamos, nas Seções 3.3 e 3.4, de que forma se dá o controle da verdade no modelo $M[G]$ pelos elementos da ordem $\mathbb{P}$. Na realidade, definimos este controle de duas formas,

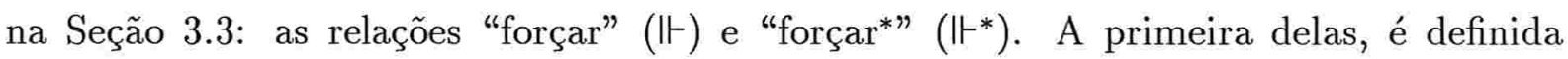
de forma que $\mathbb{P}$ ou, mais ainda, os elementos de $G \subseteq \mathbb{P}$ sejam responsáveis por como a verdade em $M[G]$ é aproximada a partir de $M$. Nesse sentido, o principal resultado é:

Teorema. $M[G]$ satisfaz uma sentença $\Phi$ se, e somente se, existe $p \in G$ tal que $p \Vdash \Phi$.

Usando os elementos de $\mathbb{P}$, definimos então objetos que são candidatos aos objetos de $M[G]$, isto é, possíveis objetos de $M[G]$. Feito isto, definimos a relação "forçar*", dentro de $M$ e mostramos, na Seção 3.4, que elas são equivalentes. Isso é interessante, pois queríamos controlar a verdade em $M[G]$, a partir de $M$. Notemos que a relação "forçar" faz o serviço de controlar a verdade em $M[G]$ e a relação "forçar*" é definida dentro de $M$. Com sua equivalência, obtemos o que queríamos.

Porém, para usarmos o forcing para mostrar a consistência de afirmações, não é necessário fazermos um estudo completo de porque, de fato, ele funciona. Se acreditamos que o modelo $M[G]$ gerado pelo forcing é um modelo para ZFC e que a relação "forçar" garante a veracidade de certas afirmações no modelo $M[G]$, basta que entendamos como axioma da escolha (o C se refere ao axioma da escolha): a axiomatização usual da matemática. Esse conjunto de axiomas foi escolhido como o mais natural para a teoria dos conjuntos. Assim, quando fazemos demonstrações, usamos as regras de lógica e os axiomas de ZFC. Veja [Ci], Apêndice A, para a lista completa de tais axiomas.

${ }^{4}$ Dizemos que uma sentença é independente se sua afirmação e sua negação são consistentes. 
funciona tal relação e como devem ser os elementos da ordem para que tenhamos válida em $M[G]$ uma dada sentença. Tentamos dar, ao longo do capítulo, explicações suficientes para isto.

Os forcing's c.c.c. e os forcing's $\sigma$-fechados possuem boas propriedades que serão destacadas na Seção 4.5. A principal delas é a preservação de cardinais, que permite-nos controlar o valor de certos cardinais (como o $2^{\omega}$ ), a fim de obter, por exemplo, que em um certo $M[G]$ vale que $2^{\omega}>\omega_{1}$.

Cohen desenvolveu o método de forcing para provar a consistência de $\neg$ CH com ZFC. O modelo que ele criou para isto, e, portanto, o primeiro modelo obtido usando o forcing, é chamado de modelo de Cohen. O forcing de Cohen (que gera tal modelo) é apresentado na Seção 4.6, com os principais resultados, incluindo o fato que ele é c.c.c. e, portanto, preserva cardinais. Mesmo não sendo utilizado neste trabalho, apresentamos o forcing de Cohen por sua importância histórica e para exemplificar quais propriedades de um forcing podem influenciar o modelo que ele gera.

Por fim, na Seção 4.7, definimos o forcing de Sacks. Este forcing será utilizado no Capítulo 7, para mostrar um resultado de consistência sobre espaços com a propriedade de Grothendieck. Ainda nesta seção, mostramos algumas propriedades interessantes deste forcing, bem como os resultados utilizados posteriormente. Notemos que o forcing de Sacks, apesar de não ser c.c.c., nem $\sigma$-fechado, preserva cardinais.

Como referência para os tópicos aqui discutidos, indicamos [Ci] para a Seção 4.1, [Ku] para as Seções 4.2, 4.3, 4.4, 4.5 e 4.6 e indicamos [Ba] e [Sa] para a Seção 4.7. Para detalhes de lógica que aqui são discutidos, porém não de forma muito rigorosa, indicamos [End]. Assumimos algum conhecimento de teoria dos conjuntos que pode ser encontrado em [Roi].

\subsection{Consistência e modelos para ZFC}

Primeiramente, lembramos que a linguagem de ZFC é formada apenas pelo símbolo $\epsilon$ (significando a relação pertencer). Assim, sentenças e fórmulas na linguagem ${ }^{5}$ de ZFC devem conter apenas tal símbolo, além de variáveis, do símbolo de igualdade $(=)$ e dos

\footnotetext{
${ }^{5}$ Para mais detalhes, veja [End].
} 
conectivos e quantificadores lógicos usuais $(\wedge, \vee, \neg, \rightarrow, \forall, \exists)$. No que segue, sempre que falarmos em sentenças e fórmulas, estaremos nos referindo a sentenças e fórmulas nesta linguagem.

Como já dissemos, o forcing é um método para provar a consistência de afirmações, isto é, para provar que se ZFC (Teoria de Zermelo-Fraenkel) é consistente, então uma certa afirmação é consistente com esta teoria. Para entender o que isto significa, temos a seguinte definição:

Definição 4.1. Dado um conjunto de sentenças $T$ na linguagem de ZFC, dizemos que uma sentença $\Phi$ é conseqüência de $T$ (e denotamos por $T \vdash \Phi$ ), se $\Phi$ pode ser provada ${ }^{6}$ a partir de $T$. Daí, dizemos que um conjunto de sentenças $T$ é inconsistente se ele leva a uma contradição, isto é, se existe uma sentença $\Phi$ tal que $T \vdash \Phi$ e $T \vdash \neg \Phi$. Daí, $T$ é dito consistente (e denotamos por Con $(T)$ ) se não é inconsistente, ou seja, se não prova nenhuma contradição. Dizemos ainda que uma sentença $\Phi$ é relativamente consistente com um conjunto de sentenças $T$ se, supondo que $T$ é consistente, temos que o conjunto $T \cup\{\Phi\}$ é consistente.

Notemos que se um conjunto de sentenças $T$ é inconsistente, então existe $\Phi$ tal que $T \vdash \Phi \wedge \neg \Phi$. Daí, existe uma prova formal de $\Phi \wedge \neg \Phi$ a partir de $T$. Como uma prova formal é uma seqüência finita, temos que existe $T_{0} \subseteq T$ finito (composto pelos elementos de $T$ envolvidos na prova formal de $\Phi \wedge \neg \Phi)$ tal que $T_{0}$ é inconsistente.

Por abuso de linguagem, muitas vezes diremos apenas que uma sentença $\Phi$ é "consistente" ao invés de dizer que $\Phi$ é "relativamente consistente" com ZFC. E de que forma o forcing ajuda a provar a consistência de afirmações, uma vez que ele fornece modelos? Vejamos:

Definição 4.2. Seja $M$ um conjunto e $\Phi$ uma fórmula. Definimos a relativização de $\Phi$ a $M$ (e denotamos por $\Phi^{M}$ ) por indução na complexidade de $\Phi$, por

(a) $(x=y)^{M}$ é $x=y$;

\footnotetext{
${ }^{6}$ Uma sentença $\Phi$ pode ser provada a partir de um conjunto de sentenças $T$ se existe uma prova formal de $\Phi$ a partir de $T$, isto é, se existe uma seqüência finita $\Phi_{1}, \ldots, \Phi_{n}$ de fórmulas tais que $\Phi_{n}=\Phi$ e cada $\Phi_{k}$ ou é um elemento de $T$, ou é um axioma lógico (incluindo as fórmulas universalmente válidas), ou existem $i, j<k$ tais que $\Phi_{j}$ é da forma $\Phi_{i} \rightarrow \Phi_{k}$. Intuitivamente, uma prova formal é uma demonstração de $\Phi$, tendo os elementos de $T$ como hipótese. Veja [End] para mais detalhes.
} 
(b) $(x \in y)^{M}$ é $x \in y$;

(c) $(\Phi \wedge \Psi)^{M}$ é $\Phi^{M} \wedge \Psi^{M}$;

(d) $(\neg \Phi)^{M}$ é $\neg\left(\Phi^{M}\right)$;

(e) $(\exists x \Phi)^{M}$ é $\exists x\left(x \in M \wedge \Phi^{M}\right)$.

Temos, portanto, que a relativização de uma fórmula $\Phi$ a $M$ é obtida substituindo-se os quantificadores $\exists x$ e $\forall x$ por $\exists x \in M$ e $\forall x \in M$. Intuitivamente, podemos pensar que $\Phi^{M}$ é a fórmula $\Phi$ olhada por alguém morando dentro de $M$ (que não enxerga fora de $M$ ).

Definição 4.3. Dados um conjunto $M$ e uma sentença $\Phi$, dizemos que $M$ satisfaz $\Phi$ ou que $\Phi$ vale em $M$ (e denotamos por $M \vDash \Phi)$ ), se vale $\Phi^{M}$. Dado ainda um conjunto de sentenças $T$, dizemos que $M$ é um modelo para $T$ (e denotamos por $M \vDash T$ ) se para toda $\Phi \in T, M \vDash \Phi$.

Assim, um modelo para um conjunto de sentenças $T$ é um conjunto que satisfaz tais sentenças. Temos então o seguinte:

Teorema 4.4 (da Completude de Gödel). Seja T um conjunto de sentenças. Então $T$ é consistente se, e somente se, existe um modelo $M$ para $T$.

Demonstração. Indicamos [End], página 128.

Convém notar que os axiomas de ZFC implicam que existe o conjunto dos naturais. Assim, se $M$ é um modelo para $\mathrm{ZFC}$, então temos neste modelo o conjunto dos naturais, bem como os dos inteiros, dos racionais, dos reais, os espaços de Banach, etc. Temos, enfim, todos os objetos matemáticos que serão aqui estudados e podemos pensar intuitivamente que $M$ é um modelo para a matemática. Na verdade, temos, para cada objeto matemático, um objeto em $M$ que tem as mesmas propriedades que este objeto matemático.

Porém, pelo teorema acima, se obtivéssemos um modelo para ZFC, teríamos provado a consistência de ZFC, sendo que, pelo Teorema da Incompletude de Gödel ${ }^{7}$, isto não pode

\footnotetext{
${ }^{7} \mathrm{O}$ Teorema da Incompletude de Gödel garante que, usando os axiomas usuais de lógica e os axiomas de ZFC, não podemos mostrar que ZFC é consistente. Portanto, sempre assumimos que ZFC é consistente. Veja [End], página 229.
} 
ser feito. Entretanto, podemos obter modelos para qualquer subconjunto finito de axiomas de ZFC e para uma demonstração, usamos apenas um número finito de tais axiomas, uma vez que uma demonstração é uma seqüência finita de passos. Assim, quando dizemos que temos um modelo para ZFC, queremos dizer, na realidade, que temos um modelo para um número finito de tais axiomas, suficientes para o que quisermos mostrar.

No forcing, trabalhamos apenas com modelos transitivos, o que facilita em diversos aspectos:

Definição 4.5. Dizemos que um conjunto $M$ é transitivo se para todo $a \in M$ tem-se que $a \subseteq M$. Daí, dizemos que $M$ é um modelo transitivo para um conjunto de sentenças $T$ se é um modelo para $T$ e é um conjunto transitivo.

Note que modelos transitivos são casos especiais de modelos. Veremos adiante que tais modelos preservam algumas propriedades importantes, por exemplo, veremos que se $M \subseteq N$ são modelos transitivos para ZFC, então uma sentença envolvendo elementos de $M$ vale em $M$ se e somente se ela vale em $N$. Assim, dado um modelo transitivo, poderemos, a partir dele, obter informações sobre outros modelos transitivos. Porém, omitiremos muitas vezes esta parte técnica.

Antes disso, vejamos que, no forcing, basta que consideremos tais modelos. O que nos garante isso é o Princípio de Forcing. Para prová-lo, precisamos ainda de alguns resultados.

Lema 4.6. Para todo subconjunto finito $T$ de axiomas de ZFC, temos que

$$
Z F C \vdash \exists M \text { um modelo transitivo e enumerável para } T \text {. }
$$

Demonstração. Indicamos [Ku], Corolário 7.11 , página 140.

Antes de mostrar o principal resultado desta seção, façamos algumas observações. Fixemos primeiramente uma fórmula $\Phi$. Suponhamos que através do forcing garantimos que para todo modelo transitivo e enumerável $M$ para $\mathrm{ZFC}$, existe um modelo transitivo e enumerável $M[G]$ para $\mathrm{ZFC}+\Phi$. Na realidade, quando provamos que o forcing fornece um modelo $M[G]$ para cada sentença de ZFC e para $\Phi$, usamos apenas o fato que $M$ é modelo para um conjunto finito de axiomas de ZFC. Em particular, se $\Phi$ é uma sentença tal que se $M$ é modelo de ZFC, então $M[G]$ é modelo para $\Phi$ (note que $\Phi$ aqui pode ser 
um axioma de ZFC), então existe um subconjunto finito $T_{\Phi}$ de axiomas de ZFC tal que se $M$ é modelo para $T_{\Phi}$, então $M[G]$ é modelo para $\Phi$. Assim, no enunciado do próximo resultado, a hipótese

"para todo modelo transitivo e enumerável $M$ para ZFC, existe um modelo transitivo e enumerável $N$ para $Z F C+\Phi$ tal que $M \subseteq N$ "

deve ser entendida como

$$
\begin{gathered}
\text { para todas } \Phi_{1}, \ldots, \Phi_{n} \text { fórmulas de } \mathrm{ZFC}+\Phi, \\
\text { existem fórmulas } \Psi_{1}, \ldots, \Psi_{k} \text { de } \mathrm{ZFC} \text { tais que } \\
\mathrm{ZFC} \vdash\left\{\begin{array}{l}
\forall M \text { modelo transitivo e enumerável para } \Psi_{1}, \ldots, \Psi_{k}, \\
\exists N \text { um modelo transitivo e enumerável para } \Phi_{1}, \ldots, \Phi_{n} .
\end{array}\right.
\end{gathered}
$$

Teorema 4.7 (Princípio de forcing). Se para todo modelo transitivo e enumerável $M$ para $Z F C$, existe um modelo transitivo e enumerável $N$ para $Z F C+\Phi$ tal que $M \subseteq N$, então temos que $\Phi$ é relativamente consistente, isto é, Con $(Z F C)$ implica $C o n(Z F C+\Phi)$.

Demonstração. Fixemos $\Phi$ uma fórmula e suponhamos que vale a hipótese do teorema. Suponhamos também que ZFC $+\Phi$ é inconsistente. Daí, existem $\Phi_{1}, \ldots \Phi_{n}$ fórmulas de $\mathrm{ZFC}+\Phi$ que provam uma contradição.

Pela hipótese do teorema, existem $\Psi_{1}, \ldots, \Psi_{k}$ fórmulas de ZFC tais que

$$
\text { ZFC } \vdash\left\{\begin{array}{l}
\forall M \text { modelo transitivo e enumerável para } \Psi_{1}, \ldots, \Psi_{k}, \\
\exists N \text { um modelo transitivo e enumerável para } \Phi_{1}, \ldots, \Phi_{n} .
\end{array}\right.
$$

Mas pelo Lema 4.6,

ZFC $\vdash \exists M$ um modelo transitivo e enumerável para $\Psi_{1}, \ldots, \Psi_{k}$.

Logo,

ZFC $\vdash \exists N$ um modelo transitivo e enumerável para $\Phi_{1}, \ldots, \Phi_{n}$.

Assim, temos que ZFC é inconsistente. Portanto, assumindo que ZFC é consistente, segue que $\mathrm{ZFC}+\Phi$ é consistente.

Pelo Teorema da Completude de Gödel (4.4), para mostrar a consistência relativa de uma sentença $\Phi$, precisaríamos mostrar que se existe um modelo para ZFC, então existe 
um modelo para $\mathrm{ZFC}+\Phi$. O resultado acima garante que, para provar isto, é suficiente provar algo mais fraco: se existe um modelo transitivo e enumerável $M$ para ZFC (uma hipótese mais forte do que existir um modelo qualquer), então existe um modelo transitivo e enumerável $N$ para $\mathrm{ZFC}+\Phi$, que contém $M$.

Para terminar esta seção, uma última definição importante:

Definição 4.8. Dado um conjunto de sentenças $T$ e uma sentença $\Phi$, dizemos que $\Phi$ é relativamente independente de $T$ se $\Phi$ e $\neg \Phi$ são ambas relativamente consistentes com $T$. Diremos também que $\Phi$ é independente se $\Phi$ é relativamente independente de $Z F C$.

\subsection{A construção de $M[G]$}

O objetivo desta seção é mostrar de que forma usaremos o forcing para obter a consistência de afirmações: construímos, a partir de um modelo transitivo e enumerável $M$ e de uma ordem parcial $\mathbb{P}$, um modelo transitivo $M[G]$. Portanto, nosso objetivo aqui é fazer esta construção. Comecemos definindo forcing:

Definição 4.9. Seja $\mathbb{P}$ uma ordem parciaß e sejam $p, q \in \mathbb{P}$. Dizemos que $p$ é uma extensão de $q$ ou simplesmente, que $p$ estende $q$, se $p \leq q$. Dizemos que $p, q \in \mathbb{P}$ são compatíveis se $\exists r \in \mathbb{P}$ que estende $p$ e $q$ e dizemos que $p, q$ são incompatíveis $(p \perp q)$ caso contrário.

Chamamos de forcing toda ordem parcial $\mathbb{P}$ tal que para todo $p \in \mathbb{P}$, existem $q_{1}, q_{2}$ incompativeis, que estendem $p$, isto é,

$$
\forall p \in \mathbb{P} \quad \exists q_{1}, q_{2} \in \mathbb{P} \quad q_{1}, q_{2} \leq p \text { e } q_{1} \perp q_{2},
$$

e existe o elemento máximo de $\mathbb{P}$ (e o denotamos por 1$)$, isto é, existe $1 \in \mathbb{P}$ tal que para todo $p \in \mathbb{P}$ temos que $p \leq 1$.

Assim, chamamos de forcing uma ordem parcial com elemento máximo, sem "partes" triviais: se existe $p \in \mathbb{P}$ tal que não existem extensões $q_{1}, q_{2}$ de $p$ incompatíveis, então $\{q \in \mathbb{P}: q \leq p\}$ é trivial.

\footnotetext{
${ }^{8}$ Uma ordem parcial é um par $\langle P, \leq\rangle$, onde $P$ é um conjunto não vazio e $\leq$ é uma relação transitiva e reflexiva entre elementos de $P$. Denotamos por $\mathbb{P} \circ$ par $\langle P, \leq\rangle$ e usamos $\mathbb{P}$ para $P$, por abuso de notação.
} 
Exemplo 4.10. Tomamos $\mathbb{P}=\left\{f \subseteq \omega_{2} \times 2\right.$ : $\operatorname{dom}(f)$ é finito e $f$ é função $\}$ com a ordem $f \leq g$ se $f \supseteq g$. Ou seja, $\mathbb{P}$ é o conjunto das funçôes finitas de $\omega_{2}$ em 2 , com $f \leq g$ se $f$ estende g como função. Claramente, temos que 1 de $\mathbb{P}$ é a função $\emptyset$, isto é, o maior elemento desta ordem parcial é $\emptyset$.

O modelo $M[G]$ será definido a partir do modelo $M$ e dos elementos da ordem $\mathbb{P}$ que pertencem a $M$. Para isto, precisamos algumas definições relacionadas à ordem $\mathbb{P}$.

Definição 4.11. Seja $\mathbb{P}$ um forcing. Dizemos que $D \subseteq \mathbb{P}$ é denso em $\mathbb{P}$ se $\forall p \in P \exists q \in$ $D(q \leq p)$, ou seja, se para todo elemento de $\mathbb{P}$, existe um elemento de $D$ que o estende.

Dizemos que $G \subseteq \mathbb{P}, G \neq \emptyset$ é um filtro em $\mathbb{P}$ se ocorrem:

- $\forall p, q \in G \exists r \in G(r \leq p \wedge r \leq q)$;

- $\forall p \in G \forall q \in \mathbb{P}(p \leq q \rightarrow q \in G)$.

Dissemos que para definir $M[G]$, iríamos usar $M$ e o forcing $\mathbb{P}$. Mas então porque esta notação $M[G]$ ? Na realidade, definiremos $M[G]$ a partir de $M$, da ordem $\mathbb{P}$ e de um filtro $G$ em $\mathbb{P}$ (o que explica a notação $M[G]$ ) com uma propriedade muito forte:

Definição 4.12. Seja $\mathbb{P} u m$ forcing e $M$ um conjunto. Dizemos que $G \subseteq \mathbb{P}$ é um $\mathbb{P}$ genérico sobre $M$ se $G$ é um filtro em $\mathbb{P}$ e para todo denso $D \subseteq \mathbb{P}$, se $D \in M$, então $G \cap D \neq \emptyset$.

Pretendemos ter um controle sobre a verdade em $M[G]$, a partir de $M$. Iremos obter tal controle através do filtro $G$. Como já dissemos, este filtro terá uma propriedade forte, que é ser $\mathbb{P}$-genérico sobre $M$. Não vimos porém, como garantiremos que, de fato, $G$ ou $\mathbb{P}$ (que estão em $M$ ) terão controle sobre este modelo $M[G]$. O que fazemos é obter, usando $M$ e $\mathbb{P}$, candidatos a elementos de $M[G]$. Assim, dados um modelo $M$ e $\mathbb{P}$ um forcing em $M$, temos um conjunto de candidatos a elementos de $M[G]$. Feito isto, veremos que $G$ decidirá quem são estes candidatos.

Definição 4.13. Seja $\mathbb{P}$ um forcing. Dizemos que $\tau$ é um $\mathbb{P}$-nome ${ }^{9}$ se $\tau$ é uma relação binária e

$$
\forall\langle\sigma, p\rangle \in \tau[\sigma \text { é um } \mathbb{P} \text {-nome e } p \in \mathbb{P}] .
$$

\footnotetext{
${ }^{9}$ Notamos que isto pode ser definido por recursão, uma vez que $\epsilon$ é uma relação bem-fundada. Veja em [Ku], Teorema 5.6, página 103, que garante isto.
} 
Denotamos por $V^{\mathbb{P}}$ a classe de todos os $\mathbb{P}$-nomes e se $M$ é um modelo transitivo de $Z F C$ com $\mathbb{P} \in M$, denotamos por $M^{\mathbb{P}}$ o conjunto $V^{\mathbb{P}} \cap M$.

Exemplo 4.14. Seja $\mathbb{P}=F n\left(\omega_{2}, 2\right)$ como no Exemplo 4.10. Temos que $\tau_{0}=\emptyset$ é um $\mathbb{P}$-nome trivialmente. E temos também que $\tau_{1}=\langle\emptyset, 1\rangle$, onde $1 \in \mathbb{P}$ é um $\mathbb{P}$-nome. Assim como $\tau_{2}=\left\{\left\langle\tau_{0}, 1\right\rangle,\left\langle\tau_{1}, 1\right\rangle\right\}$.

Os elementos de $M^{\mathbb{P}}$ são os candidatos a elementos de $M[G]$, isto é, os possíveis elementos de $M[G]$. Queremos agora, para cada um destes candidatos, decidir quem ele representará em $M[G]$. Teremos assim que cada um destes "nomes" para elementos de $M[G]$ corresponde a um específico elemento de $M[G]$. Note, porém, que podemos ter mais de um nome para cada elemento de $M[G]$, isto é, podemos decidir que dois nomes distintos são nomes para um mesmo elemento de um $M[G]$.

Quem faz esse serviço de decidir quem cada nome representa é o $\mathbb{P}$-genérico $G$. Por isso a notação $M[G]$ faz sentido: dados um modelo transitivo e enumerável $M$ para ZFC e um forcing $\mathbb{P}$ em $M$, temos definidos acima os candidatos para $M[G]$. Ao fixar $G$ um $\mathbb{P}$-genérico, temos quem são os elementos de $M[G]$ :

Definição 4.15. Sejam $\mathbb{P}$ um forcing, $G$ um filtro e $\tau$ um $\mathbb{P}$-nome. Chamamos de $\operatorname{val}(\tau, G)$ o conjunto ${ }^{10}\{\operatorname{val}(\sigma, G): \exists p \in G\langle\sigma, p\rangle \in \tau\}$.

Seja $M$ um modelo transitivo para $Z F C$ e suponha que $\mathbb{P} \in M$ e que $G$ é um $\mathbb{P}$-genérico sobre $M$. Então, chamamos de $M[G]$ o conjunto $\left\{\operatorname{val}(\tau, G): \tau \in M^{\mathbb{P}}\right\}$.

Exemplo 4.16. Seja $M$ um modelo transitivo e enumerável para $Z F C, \mathbb{P}=F n\left(\omega_{2}, 2\right) \cap$ $M \in M$ (como em 4.10), e $G$ um $\mathbb{P}$-genérico sobre $M$. Assim, temos que val $\left(\tau_{2}, G\right)=$ $2=\{0,1\}$, onde $\tau_{2}$ é como em 4.14, pois observe que $1 \in G$, qualquer que seja $G$ e, portanto, $\operatorname{val}\left(\tau_{2}, G\right)=\operatorname{val}\left(\left\{\left\langle\tau_{0}, 1\right\rangle,\left\langle\tau_{1}, 1\right\rangle\right\}, G\right)=\left\{\operatorname{val}\left(\tau_{0}, G\right), \operatorname{val}\left(\tau_{1}, G\right)\right\} . \quad E$ temos que $\operatorname{val}\left(\tau_{0}, G\right)=\operatorname{val}(\emptyset, G)=\emptyset=0 \operatorname{eval}\left(\tau_{1}, G\right)=\operatorname{val}\left(\left\{\left\langle\tau_{0}, 1\right\rangle\right\}, G\right)=\left\{\operatorname{val}\left(\tau_{0}, G\right)\right\}=\{0\}=1$. Assim, temos que $\tau_{0}$ é um candidato para o 0 de $M[G]$, ou seja, um nome para o 0 de $M[G]$ e $\operatorname{val}\left(\tau_{0}, G\right)$ é o 0 de $M[G]$. Analogamente para $\tau_{1}$ e $\tau_{2}$.

Note que dados $\tau, \sigma \mathbb{P}$-nomes, temos que $\operatorname{val}(\tau, G) \in \operatorname{val}(\sigma, G)$ se, e somente se, existe $p \in G$ tal que $\langle\tau, p\rangle \in \sigma$. Isso será expresso por $p$ força que $\tau$ pertence a $\sigma$.

\footnotetext{
${ }^{10}$ Novamente aqui usamos que $\epsilon$ é uma relação bem-fundada, para fazer a definição por recursão, como no caso de $\mathbb{P}$-nomes.
} 
Convém mencionar que, da forma como $M[G]$ foi definido, já temos que se $M$ é enumerável, então $M[G]$ também o é: para isto, basta notar que $M^{\mathbb{P}}$ é enumerável, uma vez que $\operatorname{val}(\cdot, G): M^{\mathbb{P}} \rightarrow M[G]$ é sobrejetora. Como $M^{\mathbb{P}} \subseteq M$, temos que $M^{\mathbb{P}}$ é enumerável e, assim, $M[G]$ é enumerável.

Pretendemos agora verificar que $M[G]$ possui diversas propriedades desejadas. Vimos que os elementos de $M[G]$ são definidos usando-se os elementos de $\mathbb{P}$ e notamos que os elementos de $G$ têm um papel central nesta definição: eles definem quem é, em $M[G]$, um candidato a elemento de $M[G]$. Assim, pode ser interessante saber que um certo $p \in \mathbb{P}$, também está em $G$. Para isso, temos o seguinte:

Lema 4.17. Sejam $M$ um conjunto enumerável, $\mathbb{P}$ um forcing e $p \in \mathbb{P}$. Existe $G$ um $\mathbb{P}$-genérico sobre $M$ tal que $p \in G$.

Demonstração. Como $M$ é enumerável, existem apenas enumeráveis $D \subseteq \mathbb{P}$ tais que $D \in$ $M$. Seja assim $\left(D_{n}\right)_{n \in \omega}$ uma enumeração dos densos em $\mathbb{P}$ que estão em $M$. Definimos, por indução, uma seqüência $\left(q_{n}\right)_{n \in \omega}$ tal que $q_{0}=p, q_{n+1} \leq q_{n}$ e $q_{n+1} \in D_{n}$. Podemos fazer isto pois dado $q_{n}$, existe $q_{n+1} \in D_{n} \operatorname{com} q_{n+1} \leq q_{n}$, uma vez que $D_{n}$ é denso. Daí, tomamos como $G$ o filtro gerado por $\left\{q_{n}: n \in \omega\right\}$, isto é, $G=\left\{p^{\prime} \in P: \exists n\left(p^{\prime} \leq q_{n}\right)\right\}$. Assim, $p \in G$ e $G$ é $\mathbb{P}$-genérico sobre $M$.

Outra propriedade que gostaríamos que $M[G]$ tivesse, é que $M[G] \neq M$. O que garante isto é $\mathbb{P}$-genérico $G$ : veremos adiante que $G \in M[G]$ e o próximo resultado mostra que $G \notin M:$

Lema 4.18. Seja $M$ um modelo transitivo e enumerável para $Z F C$ e $\mathbb{P}$ um forcing. Se $\mathbb{P} \in M$ e $G$ é um $\mathbb{P}$-genérico sobre $M$, então $G \notin M$.

Demonstração. Suponha por absurdo que $G \in M$. Então $D=\mathbb{P} \backslash G \in M$, já que $\mathbb{P}, G \in M$ e $M$, sendo modelo transitivo para ZFC, é fechado pelas operações conjuntísticas . Vamos provar que $D$ é denso em $\mathbb{P}$. Considere $p \in \mathbb{P}$. Então existem $q, r \in \mathbb{P}$ tais que $q \leq p$, $r \leq p$ e $q \perp r$. Mas daí não vale que $q, r \in G$, já que $G$ é filtro e, portanto, todos os seus elementos são dois a dois compatíveis. Logo, pelo menos uma das extensões não pertence a $G$, isto é, pertence a $D$. Com isso, temos que $D$ é denso e que $D \cap G=\emptyset$ (pela definição de $D$ ), contradizendo o fato que $G$ é $\mathbb{P}$-genérico sobre $M$. 
Para obter mais propriedades de $M[G]$, precisamos definir um conceito de lógica bastante útil aqui:

Definição 4.19. Sejam $M, N$ modelos para $Z F C$ tais que $M \subseteq N$. Dizemos que uma fórmula $\Phi$ é absoluta para $M, N$ se

$$
\forall x_{1}, \ldots, x_{n} \in M\left(\Phi^{M}\left(x_{1}, \ldots, x_{n}\right) \leftrightarrow \Phi^{N}\left(x_{1}, \ldots, x_{n}\right)\right)
$$

Dizemos ainda que uma fórmula é absoluta para $M$ se

$$
\forall x_{1}, \ldots, x_{n} \in M\left(\Phi^{M}\left(x_{1}, \ldots, x_{n}\right) \leftrightarrow \Phi\right) .
$$

A absolutividade nos servirá para passar de fórmulas que valem em $M$ a fórmulas que valem em $M[G]$ e vice-versa. O fato que $M$ e $M[G]$ são transitivos, garantem que muitas fórmulas são absolutas para $M, M[G]$. É claro que muitas outras não são absolutas, senão não conseguiríamos mudar nada ao passar de $M$ para $M[G]$. Não entraremos nestes detalhes, mas isso pode ser encontrado em [Ku], Capítulo 4, Seção 3. Enunciamos apenas alguns exemplos importantes:

Proposição 4.20. Sejam $\mathbb{P}$ um forcing, $M, N$ modelos transitivos para $Z F C$ tais que $\mathbb{P} \in M \subseteq N$. Então:

- "T é um $\mathbb{P}$-nome" é absoluta para $M, N$ e para $M$;

- se $G$ é um $\mathbb{P}$-genérico sobre $M$, então “ $x$ é val $(\tau, G)$ ” é absoluta para $M, N$ e para $M$.

Demonstração. Veja [Ku], Capítulo 4, Seção 3.

Note que, desta proposição, podemos concluir que

$$
M^{\mathbb{P}}=\left\{\tau \in M:(\tau \text { é um } \mathbb{P} \text {-nome })^{M}\right\} .
$$

Temos definido $M[G]$ e já sabemos que $G \notin M$, se $G$ é um $\mathbb{P}$-genérico sobre $M$. Para poder utilizar o Princípio de Forcing (4.7), precisamos que $M[G]$ tenha algumas propriedades: que $M \subseteq M[G]$ e que $M[G]$ seja um modelo transitivo e enumerável para ZFC (enumerável já temos). Para justificar a notação $M[G]$ gostaríamos também que $G \in M[G]$. 
Para mostrar que $M \subseteq M[G]$, precisamos achar, para cada elemento de $M$, um candidato a elemento de $M[G]$, isto é, um $\mathbb{P}$-nome, tal que $\operatorname{val}(\tau, G)$ seja este elemento. Para isso, definimos:

Definição 4.21. Seja $\mathbb{P}$ um forcing. Definimos o $\mathbb{P}$-nome $\check{x}$ recursivamente ${ }^{11}$ como $\check{x}=$ $\{\langle\check{y}, 1\rangle: y \in x\}$.

Exemplo 4.22. Em 4.14 temos que $\tau_{0}, \tau_{1}$ e $\tau_{2}$ são iguais a $\check{0}, \check{1}$ e $\check{2}$ respectivamente.

Notemos que no último exemplo temos que $0=\operatorname{val}(\check{0}, G), 1=\operatorname{val}(\check{1}, G)$ e $2=$ $\operatorname{val}(\check{2}, G)$. Vejamos que isso não é uma coincidência. De fato, temos que se $x \in M$, então $\operatorname{val}(\check{x}, G)=x$ e portanto, $x \in M[G]$. Para provar isso, precisamos mais um resultado de absolutividade:

Proposição 4.23. Sejam $\mathbb{P}$ um forcing, $M, N$ modelos transitivos para ZFC tais que $\mathbb{P} \in M \subseteq N$. Então " $\tau$ é $\check{x}$ ” é absoluta para $M, N$ e para $M$.

Demonstração. Veja [Ku], Capítulo 4, Seção 3.

Vejamos enfim que $M \subseteq M[G]$ :

Lema 4.24. Seja $M$ um modelo transitivo de $Z F C, \mathbb{P}$ um forcing tal que $\mathbb{P} \in M$ e $G$ um filtro não vazio qualquer sobre $\mathbb{P}$. Então:

(i) $\forall x \in M\left[\check{x} \in M^{\mathbb{P}} \wedge \operatorname{val}(\check{x}, G)=x\right]$

(ii) $M \subseteq M[G]$

Demonstração. (i) Pela absolutividade de $\check{x}$, temos que $\check{x} \in M^{\mathbb{P}}$. Já para provarmos que $\operatorname{val}(\check{x}, G)=x$, basta usar indução sobre $x$ e notar que $\operatorname{val}(\check{x}, G)=\{\operatorname{val}(\check{y}, G)$ : $y \in x\}$.

(ii) Imediato a partir do item anterior.

\footnotetext{
${ }^{11}$ Notamos novamente que, como no caso de $\mathbb{P}$-nome, isto pode ser definido por recursão, uma vez que $\in$ é uma relação bem-fundada. Veja em [Ku], Teorema 5.6, página 103, que garante isto.
} 
Em resumo, temos que para cada elemento de $M[G]$, podem existir vários nomes, mas, para cada elemento $x$ de $M$ (e note que $M \subseteq M[G]$ ), temos um nome canônico para $x$, o nome $\check{x}$.

Estamos agora interessados em provar que $G \in M[G]$. Como fizemos para cada elemento de $M$, encontraremos um $\mathbb{P}$-nome $\Gamma$ tal que $\operatorname{val}(\Gamma, G)=G$ e, portanto, $G \in M[G]$. Neste caso porém, não podemos fazê-lo como no caso de elementos de $M$, uma vez que $G$ não está em $M$ e $\breve{G}$ definido da mesma forma não estaria em $M$. Vejamos qual é a saída neste caso:

Definição 4.25. Seja $\mathbb{P}$ um forcing. Então chamamos de $\Gamma$ o nome $\{\langle\check{p}, p\rangle: p \in \mathbb{P}\}$.

Lema 4.26. Seja $M$ um modelo transitivo para $Z F C, \mathbb{P}$ um forcing tal que $\mathbb{P} \in M$ e $G$ um filtro não vazio qualquer sobre $\mathbb{P}$. Então, val $(\Gamma, G)=G$ e, portanto, $G \in M[G]$.

Demonstração.

$\operatorname{val}(\Gamma, G)=\operatorname{val}(\{\langle\check{p}, p\rangle: p \in \mathbb{P}\}, G)=\{\operatorname{val}(\check{p}, G): p \in G\}=\{p: p \in G\}=G$.

Resta ver ainda que $M[G]$ é transitivo e que é modelo para ZFC. A transitividade de $M[G]$ está garantida desde o momento em que definimos $M[G]$, uma vez que os $\operatorname{val}(\tau, G)$ foram definidos por recursão e assim, elementos de $\operatorname{val}(\tau, G)$ são da forma $\operatorname{val}(\sigma, G)$ com $\sigma \in M^{\mathbb{P}}$ :

Lema 4.27. Seja $M$ um modelo transitivo para $Z F C, \mathbb{P}$ um forcing tal que $\mathbb{P} \in M$ e $G$ um filtro não vazio qualquer sobre $\mathbb{P}$. Então $M[G]$ é transitivo.

Demonstração. Temos que mostrar que se $a \in M[G]$ e $b \in a$ então $b \in M[G]$. Mas se $a \in$ $M[G]$, então $a=\operatorname{val}(\sigma, G)$, onde $\sigma \in M^{\mathbb{P}}$. Assim, $a=\{\operatorname{val}(\pi, G): \exists p \in G,\langle\pi, p\rangle \in \sigma\}$. Como $b \in a$, então $b=\operatorname{val}(\pi, G)$ para algum $\pi \in M^{\mathbb{P}}$, ou seja, $b \in M[G]$.

Vejamos então que $M[G]$ está contido em todo modelo transitivo que contém $M$ e ao qual $G$ pertence.

Lema 4.28. Seja $M$ um modelo transitivo de $Z F C, \mathbb{P}$ um forcing tal que $\mathbb{P} \in M$ e $G$ um $\mathbb{P}$-genérico sobre $M$. Se $N$ é um modelo transitivo de ZFC tal que $N \supseteq M$ e $G \in N$, então $M[G] \subseteq N$. 
Demonstração. Seja $\operatorname{val}(\tau, G) \in M[G]$. Então $\tau \in M^{\mathbb{P}}$ e, portanto, $\tau \in N^{\mathbb{P}}$, uma vez que "ser um $\mathbb{P}$-nome" é absoluta para modelos transitivos. Como $G \in N$ e "ser $\operatorname{val}(\tau, G)$ " é transitiva para modelos transitivos, temos que $\operatorname{val}(\tau, G)=(\operatorname{val}(\tau, G))^{N} \in N$.

O próximo teorema garante que $M[G]$ é um modelo para ZFC. Sua demonstração não será feita aqui, uma vez que nosso objetivo é aplicar o forcing. Note, porém, que usamos que $G$ deve ser um $\mathbb{P}$-genérico sobre $M$ apenas para garantir que $G \notin M$. Esta hipótese também é necessária para este resultado:

Teorema 4.29. Seja $\mathbb{P}$ um forcing, $M$ um modelo transitivo e enumerável para ZFC tal que $\mathbb{P} \in M$ e $G$ um $\mathbb{P}$ genérico sobre $M$. Então, $M[G]$ é um modelo para $Z F C$.

Demonstração. Indicamos [Ku], Capítulo 7, Seção 4.

Temos assim que se $M$ é um modelo transitivo e enumerável para ZFC, $\mathbb{P} \in M$ um forcing e $G$ um $\mathbb{P}$-genérico sobre $M$, então $M[G]$ é um modelo transitivo e enumerável para ZFC tal que $M \subseteq M[G], G \in M[G]$ e que $M[G]$ é o menor com tais propriedades.

\subsection{As relações $\Vdash \mathrm{e} \mathbb{}^{*}$}

Com os resultados da seção anterior, estamos em condições de aplicar o Princípio de Forcing (4.7) para obter a consistência de uma fórmula $\Phi$, desde que possamos garantir de alguma forma que se $M$ é um modelo transitivo e enumerável para ZFC, então $M[G]$ é um modelo para $\Phi$. Já sabemos que se $M$ é um modelo transitivo e enumerável para ZFC, então $M[G]$ também o é e que $M \subseteq M[G]$.

Desde o início da seção anterior, estávamos preocupados em garantir que $\mathbb{P}$ (ou $M$ ) tivesse um controle sobre a verdade em $M[G]$. Assim, se tivemos sucesso nesta tentativa, seria de se esperar que pudéssemos garantir que alguma fórmula $\Phi$ vale em $M[G]$, quando escolhemos $G$ para isso.

Pretendemos agora entender de que forma os elementos de $\mathbb{P}$ fornecem informações sobre a verdade em $M[G]$, a fim de poder garantir que se escolhemos $G$ convenientemente, então $\Phi$ vale em $M[G]$. Para isto, definiremos as relações $\Vdash$ (forçar) e $\Vdash^{*}$ (forçar-estrela), 
que fazem esta ligação entre as sentenças e os elementos de $\mathbb{P}$ e, dependendo de como eles se relacionam, temos a verdade ou falsidade das sentenças em $M[G]$.

Definiremos $\Vdash$ utilizando a verdade em $M[G]$ e, portanto, $\Vdash$ terá um controle sobre esta. Por outro lado, definiremos $\mathbb{1}^{*}$ diretamente usando propriedades de $\mathbb{P}$. Assim, $\mathbb{1}^{*}$ será definível dentro de $M$. Ao mostrarmos que ambas coincidem (na Seção 4.4), teremos um controle sobre a verdade em $M[G]$ a partir de propriedades da ordem $\mathbb{P}$, que são propriedades de elementos de $M$.

Convém mencionar, primeiramente, que a linguagem do forcing é formada por todos os símbolos da linguagem de ZFC, acrescida de um conjunto de constantes. Precisamos ter, na linguagem do forcing, uma constante para cada $\mathbb{P}$-nome. Usaremos, porém, o próprio $\mathbb{P}$-nome como constante, para entender do que estamos falando. No que segue, sempre que tivermos sentenças e fórmulas depois do símbolo de forcing $\left(\Vdash^{-} \mathrm{e} \Vdash^{*}\right)$, estamos nos referindo a sentenças e fórmulas nesta linguagem.

Definição 4.30. Sejam $\phi\left(x_{1}, \ldots, x_{n}\right)$ uma fórmula, $M$ um modelo transitivo enumerável para $Z F C, \mathbb{P}$ um forcing em $M, \tau_{1}, \ldots, \tau_{n} \in M^{\mathbb{P}}$ e $p \in \mathbb{P}$. Dizemos que $p \Vdash_{\mathbb{P}, M} \phi\left(\tau_{1}, \ldots, \tau_{n}\right)$ $\left(p\right.$ força $\left.\phi\left(\tau_{1}, \ldots, \tau_{n}\right)\right)$ se

$$
\forall G\left[(G \text { é } \mathbb{P} \text {-genérico sobre } M \wedge p \in G) \rightarrow \phi^{M[G]}\left(\operatorname{val}\left(\tau_{1}, G\right), \ldots, \operatorname{val}\left(\tau_{n}, G\right)\right)\right] .
$$

Quando $M$ e $\mathbb{P}$ estiverem claros no contexto, usaremos $\Vdash$ no lugar de $\Vdash_{M, \mathbb{P}}$.

Esta relação nos diz quais sentenças são válidas em $M[G]$, quando um certo elemento $p \in G$. Notemos, por exemplo, que se para todo $p \in \mathbb{P}$ temos que $p \Vdash \Phi$, então para todo $G \mathbb{P}$-genérico sobre $M$, temos $(\Phi)^{M[G]}$. Na verdade, uma hipótese mais fraca é suficiente para garantir isto: basta que o conjunto $\{p \in \mathbb{P}: p \Vdash \Phi\}$ seja denso em $\mathbb{P}$, uma vez que todo filtro $\mathbb{P}$-genérico intercepta todo denso. Pretendemos assim garantir que os elementos de $\mathbb{P}$ sabem (ou dizem) bastante sobre a verdade em $M[G]$. Se muitos deles dizem que algo vale em $M[G]$ é porque, de fato, aquilo vale em $M[G]$.

Provaremos adiante que dados $M$ e $G$, temos que uma sentença $\Phi$ vale em $M[G]$ se, e somente se, existe $p \in G$ tal que $p \Vdash \Phi$. A volta segue diretamente da definição acima. Assim, temos que para mostrar que uma sentença é verdadeira em $M[G]$, basta achar $p \in G$ tal que $p \Vdash \Phi$. Usando o fato que as relações $\Vdash$ e $\Vdash^{*}$ são equivalentes, basta achar $p \in G$ tal que $p \mathbb{\Vdash}^{*} \Phi$, e isto será possível, dependendo das propriedades de $\mathbb{P}$. 
Vejamos algumas propriedades importantes desta relação:

Lema 4.31. Seja $M$ um modelo transitivo enumerável para $Z F C, \mathbb{P}$ um forcing em $M$, $\tau_{1}, \ldots, \tau_{n} \in M^{\mathbb{P}}$ e $p \in \mathbb{P}$. Então:

(i) $\left(p \Vdash \phi\left(\tau_{1}, \ldots, \tau_{n}\right) \wedge q \leq p\right) \Rightarrow q \Vdash \phi\left(\tau_{1}, \ldots, \tau_{n}\right)$.

(ii) $\left(p \Vdash \phi\left(\tau_{1}, \ldots, \tau_{n}\right) \wedge p \Vdash \psi\left(\tau_{1}, \ldots, \tau_{n}\right)\right) \Leftrightarrow p \Vdash \phi\left(\tau_{1}, \ldots, \tau_{n}\right) \wedge \psi\left(\tau_{1}, \ldots, \tau_{n}\right)$.

Demonstração. (i) Temos que $p \Vdash \phi\left(\tau_{1}, \ldots, \tau_{n}\right)$ e $q \leq p$. Seja $G$ um $\mathbb{P}$-genérico sobre $M$ com $q \in G$. Então, temos que $p \in G$, pois $q \leq p$ e $G$ é filtro. Assim, temos que vale $\phi^{M[G]}\left(\operatorname{val}\left(\tau_{1}, G\right), \ldots, \operatorname{val}\left(\tau_{n}, G\right)\right)$ e, portanto, $q \Vdash \phi\left(\tau_{1}, \ldots, \tau_{n}\right)$.

(ii) Basta notar que $\left(\phi^{M[G]}\left(\operatorname{val}\left(\tau_{1}, G\right), \ldots, \operatorname{val}\left(\tau_{n}, G\right)\right) \wedge \psi^{M[G]}\left(\operatorname{val}\left(\tau_{1}, G\right), \ldots, \operatorname{val}\left(\tau_{n}, G\right)\right)\right)$ $\Leftrightarrow\left(\phi\left(\operatorname{val}\left(\tau_{1}, G\right), \ldots, \operatorname{val}\left(\tau_{n}, G\right)\right) \wedge \psi\left(\operatorname{val}\left(\tau_{1}, G\right), \ldots, \operatorname{val}\left(\tau_{n}, G\right)\right)\right)^{M[G]}$.

Com o item (i) deste resultado, temos que se $q \leq p$ e $p$ força uma fórmula $\Phi$, então $q$ também força esta mesma fórmula. Logo, $q$ força tudo o que $p$ força e mais coisas. Entendemos assim que, quando $q \leq p$, então $q$ é mais forte que $p$, isto é, $q$ nos dá mais informações sobre $M[G]$ do que $p$, se ambos estiverem no $\mathbb{P}$-genérico $G$. $O$ item (ii) acima nos garante apenas que vale algo que já esperávamos, intuitivamente, que valesse.

Como já dissemos, pretendemos definir a relação It* dentro de $M$. Para isso, usaremos propriedades da estrutura de $\mathbb{P}$.

Definição 4.32. Seja $\mathbb{P} u m$ forcing, $p \in \mathbb{P} e D \subseteq \mathbb{P}$. Dizemos que $D$ é denso abaixo de $p$ se

$$
\forall q \leq p \exists r \leq q(r \in D)
$$

Nossa definição de $\Vdash$ exige conhecimento sobre todos os $G$ possíveis e, portanto, não é feita dentro de $M$. Como queremos fazer algo definível em $M$, precisamos definir algo diferente (note que por 4.18, temos que $G \notin M$ e assim, nossa nova definição não pode exigir conhecimento sobre $G$ ). Para isso, definiremos $\mathbb{1}^{*}$ e, mais adiante, mostraremos a relação entre $\Vdash^{*}$ e $\Vdash$.

Pretendemos que um elemento $p \in \mathbb{P}$ force* ${ }^{*}$ uma fórmula se para todo $\mathbb{P}$-genérico sobre $M, G$ tal que $p \in G$, tem-se que $M[G]$ satisfaz $\Phi$. Queremos isso, pois queremos 
que $\mathbb{1}^{*}$ seja equivalente a $\Phi$. Mas queremos definir $\mathbb{}^{*}$ dentro de $M$. Tendo em vista os comentários que fizemos de que se $\{p \in \mathbb{P}: p \Vdash \Phi\}$ é denso em $\mathbb{P}$, então $M[G]$ satisfaz $\Phi$, definimos $\Vdash^{*}$ da seguinte maneira:

Definição 4.33. Seja $\mathbb{P}$ uma ordem parcial. Definimos $p \mathbb{1}^{*} \phi\left(\tau_{1}, \ldots, \tau_{n}\right)$ por indução na complexidade de $\phi$, onde $p \in \mathbb{P}, \phi\left(\tau_{1}, \ldots, \tau_{n}\right)$ é uma fórmula e $\tau_{1}, \ldots, \tau_{n} \in V^{\mathbb{P}}$ de forma que:

(a) $p \Vdash^{*} \tau_{1}=\tau_{2}$ se:

(i) para todo $\left\langle\pi_{1}, s_{1}\right\rangle \in \dot{\tau}_{1}$

$$
\left\{q \leq p: q \leq s_{1} \rightarrow \exists\left\langle\pi_{2}, s_{2}\right\rangle \in \tau_{2}\left(q \leq s_{2} \wedge q \Vdash^{*} \pi_{1}=\pi_{2}\right)\right\}
$$

é denso abaixo de $p$.

(ii) para todo $\left\langle\pi_{2}, s_{2}\right\rangle \in \tau_{2}$

$$
\left\{q \leq p: q \leq s_{2} \rightarrow \exists\left\langle\pi_{1}, s_{1}\right\rangle \in \tau_{1}\left(q \leq s_{1} \wedge q \Vdash^{*} \pi_{1}=\pi_{2}\right)\right\}
$$

é denso abaixo de $p$.

(b) $p \mathbb{H}^{*} \tau_{1} \in \tau_{2} s e$

$$
\left\{q \in \mathbb{P}: \exists\langle\sigma, s\rangle \in \tau_{2}\left(q \leq s \wedge q \Vdash^{*} \pi=\tau_{1}\right)\right\}
$$

é denso abaixo de p.

(c) $p \mathbb{R}^{*}\left(\phi\left(\tau_{1}, \ldots, \tau_{n}\right) \wedge \psi\left(\tau_{1}, \ldots, \tau_{n}\right)\right) s e$

$$
p \Vdash^{*} \phi\left(\tau_{1}, \ldots, \tau_{n}\right) \text { e } p \Vdash^{*} \psi\left(\tau_{1}, \ldots, \tau_{n}\right) .
$$

(d) $p \Vdash^{*} \neg \phi\left(\tau_{1}, \ldots, \tau_{n}\right)$ se não existe $q \leq p$ tal que $q \Vdash^{*} \phi\left(\tau_{1}, \ldots, \tau_{n}\right)$.

(e) $p \Vdash^{*} \exists x \phi\left(x, \tau_{1}, \ldots, \tau_{n}\right) s e$

$$
\left\{r \in \mathbb{P}: \exists \sigma \in V^{\mathbb{P}}\left(r \Vdash^{*} \phi\left(\sigma, \tau_{1}, \ldots, \tau_{n}\right)\right)\right\}
$$

é denso abaixo de p. 
Na definição de forçar*, podemos pensar que estamos trabalhando com uma hierarquia bem comportada de nomes ${ }^{12}$, isto é, podemos pensar que os elementos de um nome têm grau menor que o nome em questão. Assim, fórmulas atômicas sobre nomes podem ser reduzidas a fórmulas mais complexas sobre nomes de posto menor. Daí, para definir o que significa forçar* para uma fórmula sobre alguns nomes, assumimos que já sabemos o que significa forçar* para todas as fórmulas sobre nomes de posto menor que os nomes envolvidos naquela fórmula.

Notemos ainda que teremos que um elemento $p \in \mathbb{P}$ force* uma fórmula se muitas extensões dela forçam* esta mesma fórmula. Logo, devem haver muitos $q \leq p$ que forcem* a fórmula. Isso garantirá que um $\mathbb{P}$-genérico intercepte o conjunto de tais elementos e que, portanto, tal fórmula vale em $M[G]$.

Uma vez que definimos a relação $\mathbb{I}^{*}$ usando os conjuntos densos abaixo de um elemento $p \in \mathbb{P}$, precisaremos freqüentemente trabalhar com tais conjuntos. Já havíamos notado que estes conjuntos também têm um papel interessante na relação $\Vdash$. Vejamos então algumas propriedades destes conjuntos que serão utilizadas:

Lema 4.34. Sejam $\mathbb{P}$ um forcing, $p \in \mathbb{P}$ e $D \subseteq \mathbb{P}$. Então:

(i) se $D$ é denso abaixo de $p$ e $r \leq p$, então $D$ é denso abaixo de $r$;

(ii) se o conjunto $\{r \in \mathbb{P}: D$ é denso abaixo de $r\}$ é denso abaixo de $p$, então $D$ é denso abaixo de $p$.

Demonstração. (i) Seja $q \leq r$. Como $r \leq p$, temos $q \leq p$ e, portanto, existe $s \leq q$ tal que $s \in D$, pois $D$ é denso abaixo de $p$.

(ii) Seja $q \leq p$. Então existe $r \leq q$ tal que $D$ é denso abaixo de $r$. Logo, existe $s \in D$ tal que $s \leq r$. Assim, $s \in D$ e $s \in q$.

O item (i) do resultado acima garante que se $p$ força* uma fórmula e $q \leq p$, então $q$ força* esta mesma fórmula. Convém lembrar que já tínhamos obtido esta mesma propriedade sobre $\Vdash$, no Lema 4.31, item (i). Já o item (ii) diz que, dado $p \in \mathbb{P}$, se o conjunto dos $r$ que forçam* uma fórmula $\Phi$ é denso abaixo de $p$, então $p$ força* $^{*} \Phi$.

\footnotetext{
${ }^{12} \mathrm{O}$ que garante isto é o fato que a relação $\in$ é bem-fundada. Veja [Ku], Capítulo 3.
} 
Vejamos algumas relações entre conjunto densos e $\mathbb{P}$-genéricos. O item (i) do lema abaixo garante, por exemplo, que se $E$ é uma anticadeia maximal em $\mathbb{P}$, então $G \cap E \neq \emptyset$. Veremos que isso dá às anticadeias maximais um papel importante. O item (ii), por sua vez, garante que um $\mathbb{P}$-genérico não só intercepta os densos em $\mathbb{P}$, mas intercepta também os densos abaixo de cada elemento de $G$ :

Lema 4.35. Sejam $M$ um modelo transitivo de $Z F C, \mathbb{P} \in M$ um forcing, $E \subseteq \mathbb{P}$ com $E \in M$. Seja $G$ um $\mathbb{P}$-genérico sobre $M$. Então:

(i) ou $(G \cap E \neq \emptyset)$, оu $(\exists q \in G \forall r \in E(r \perp q))$;

(ii) se $p \in G$ e E é denso abaixo de $p$, então $G \cap E \neq \emptyset$.

Demonstração. (i) Seja

$$
D=\{p \in \mathbb{P}: \exists r \in E(p \leq r)\} \cup\{q \in \mathbb{P}: \forall r \in E(r \perp q)\} .
$$

Note que $D$ é denso, pois seja $q \in \mathbb{P}$ com $q \notin D$. Seja $r \in E$, tal que $r, q$ são compatíveis, isto é, existe $p \in \mathbb{P}$ tal que $p \leq r$ e $p \leq q$. Como $r \in E$, temos que $p \in D$.

Como $D$ é denso, $G \cap D \neq \emptyset$. Assim, tomando $r \in G \cap D$, temos que ou $\exists p \in E$ tal que $p \leq r$ e daí $p \in G$, pois $G$ é filtro, ou $p \perp q$ para todo $q \in E$.

(ii) Seja $p \in G$ e suponha que $E$ é denso abaixo de $p$. Suponha por absurdo que $E \cap G=\emptyset$. Pelo item anterior, temos que existe $q \in G$ tal que $\forall r \in E(q \perp r)$. Seja $q^{\prime} \in G$ tal que $q^{\prime} \leq q$ e $q^{\prime} \leq p$ (existe pois $G$ é filtro). Daí, como $E$ é denso abaixo de $p$, existe $r \in E$ tal que $r \leq q^{\prime}$. Portanto, $r \leq q$ e $r \leq r$, contrariando o fato que $q \perp r$.

Temos assim uma lista de propriedades que já relacionam, de alguma forma, as relações $\Vdash$ e $\mathbb{}^{*}$ : tais propriedades ao menos ilustram que elas se comportam de forma parecida.

Por fim, para terminar esta seção, vejamos algumas propriedades de $\Vdash^{*}$, que utilizaremos posteriormente.

Lema 4.36. São equivalentes: 
(i) $p \mathbb{\Vdash}^{*} \phi\left(\tau_{1}, \ldots, \tau_{n}\right)$.

(ii) $\forall r \leq p\left(r \Vdash^{*} \phi\left(\tau_{1}, \ldots, \tau_{n}\right)\right)$.

(iii) $\left\{r \in \mathbb{P}: r \mathbb{1}^{*} \phi\left(\tau_{1}, \ldots, \tau_{n}\right)\right\}$ é denso abaixo de $p$.

Demonstração. $(i) \Rightarrow(i i)$ : Suponha $\phi$ da forma $\tau_{1}=\tau_{2}$ ou $\tau_{1} \in \tau_{2}$. Então, só precisamos mostrar que os conjuntos nas definições de $r \Vdash^{*} \tau_{1}=\tau_{2}$ e $r \Vdash^{*} \tau_{1} \in \tau_{2}$ são densos abaixo de $p$. Mas como $r \leq p$, por 4.34, temos o resultado. Por indução sobre a complexidade de $\phi$ provamos o resultado.

(ii) $\Rightarrow($ iii): Imediato.

(iii) $\Rightarrow($ i $)$ : Suponha $\phi$ da forma $\tau_{1}=\tau_{2}$ ou $\tau_{1} \in \tau_{2}$. Então, os conjuntos nas definições de $r \Vdash^{*} \tau_{1}=\tau_{2}$ e $r \Vdash^{*} \tau_{1} \in \tau_{2}$ são densos abaixo de $r$. Assim, por 4.34, eles são densos abaixo de $p$. Logo, $p \Vdash^{*} \tau_{1}=\tau_{2}$ e $p \mathbb{1}^{*} \tau_{1} \in \tau_{2}$. Por indução sobre a complexidade $\phi$, provamos o resultado .

Convém notar que a relação $\Vdash$ possui propriedades análogas às acima enunciadas. Por exemplo, se $p \in \mathbb{P}$ e $p \Vdash \Phi$, então vimos que para todo $q \leq p$, temos $q \Vdash \Phi$. É claro que se todo elemento abaixo de $p$ força $\Phi$, então o conjunto dos elementos abaixo de $p$ que forçam $\Phi$ é denso abaixo de $p$. Mas será que vale também que se o conjunto dos elementos abaixo de $p$ que forçam $\Phi$ é denso abaixo de $p$, então $p$ força $\Phi$ ? Veremos adiante que sim.

\subsection{A equivalência das relações $\Vdash$ e $\Vdash^{*}$}

Estamos interessados em entender a relação entre $\mathbb{I}^{*}$ e $\Vdash$ : pretendemos provar sua equivalência. Como $\Vdash$ está relacionado com a verdade em $M[G]$, vejamos como $\mathbb{1}^{*}$ se relaciona com a verdade em $M[G]$. Este resultado prova o lado mais difícil da equivalência:

Lema 4.37. Seja $\phi\left(x_{1}, \ldots, x_{n}\right)$ uma fórmula. Sejam $M$ um modelo transitivo para $Z F C$, $\mathbb{P} u m$ forcing em $M, \tau_{1}, \ldots, \tau_{n} \in M^{\mathbb{P}}$ e seja $G$ um $\mathbb{P}$-genérico sobre $M$. Então:

(i) Se $p \in G$ e $\left(p \mathbb{1}^{*} \phi\left(\tau_{1}, \ldots, \tau_{n}\right)\right)^{M}$, então $\left(\phi\left(\operatorname{val}\left(\tau_{1}, G\right), \ldots, \operatorname{val}\left(\tau_{n}, G\right)\right)\right)^{M[G]}$.

(ii) $\operatorname{Se}\left(\phi\left(\operatorname{val}\left(\tau_{1}, G\right), \ldots, \operatorname{val}\left(\tau_{n}, G\right)\right)\right)^{M[G]}$, então existe $p \in G$ tal que $\left(p \Vdash^{*} \phi\left(\tau_{1}, \ldots, \tau_{2}\right)\right)^{M}$. 
Demonstração. Não faremos aqui todos os casos. Para a demonstração completa, indicamos [Ku], Teorema 3.5, página 197.

Suponhamos que já temos o resultado para fórmulas envolvendo nomes "menores"13" que os envolvidos na fórmula que vamos provar.

Provemos, por exemplo, o resultado para $\phi$ da forma $\tau_{1}=\tau_{2}$, assumindo que para nomes "menores" que $\tau_{1}$ e $\tau_{2}$, já temos o resultado:

(i) Suponha $p \in G$ e $p \Vdash^{*} \tau_{1}=\tau_{2}$. Queremos provar que $\operatorname{val}\left(\tau_{1}, G\right)=\operatorname{val}\left(\tau_{2}, G\right)$. Para isso, provemos que $\operatorname{val}\left(\tau_{1}, G\right) \subseteq \operatorname{val}\left(\tau_{2}, G\right)$ e $\operatorname{val}\left(\tau_{1}, G\right) \supseteq \operatorname{val}\left(\tau_{2}, G\right)$.

Assim, seja $\operatorname{val}(\pi, G) \in \operatorname{val}\left(\tau_{1}, G\right)$, onde $\left\langle\pi_{1}, s_{1}\right\rangle \in \tau_{1}$, para algum $s_{1} \in G$ (os elementos de $\operatorname{val}\left(\tau_{1}, G\right)$ são desta forma). Como $p, s_{1} \in G$, existe $r \in G \operatorname{com} r \leq p, s_{1}$. Daí, $r \Vdash^{*} \tau_{1}=\tau_{2}$, pelo Lema 4.36. Então, pela definição de $\Vdash^{*}$, temos que

$$
\left\{q \leq r: q \leq s_{1} \rightarrow \exists\left\langle\pi_{2}, s_{2}\right\rangle \in \tau_{2}\left(q \leq s_{2} \wedge q \Vdash^{*} \pi_{1}=\pi_{2}\right)\right\}
$$

é denso abaixo de $r$. Como $r \in G$, pelo Lema 4.35, temos que existe $q \in G$ tal que $g \leq r$ e $q \leq s_{1}$ implica que

$$
\exists\left\langle\pi_{2}, s_{2}\right\rangle \in \tau_{2}\left(q \leq s_{2} \wedge q \Vdash^{*} \pi_{1}=\pi_{2}\right) .
$$

Como $q \leq s_{1}$, fixemos $\left\langle\pi_{2}, s_{2}\right\rangle$ com em $\left(^{*}\right)$. Assim, $s_{2} \in G$, pois $q \leq s_{2}$ e daí, $\operatorname{val}\left(\pi_{2}, G\right) \in$ $\operatorname{val}\left(\tau_{2}, G\right)$. Por outro lado, $q \mathbb{1}^{*} \pi_{1}=\pi_{2}$ e daí, pela hipótese de indução, $\operatorname{val}\left(\pi_{1}, G\right)=$ $\operatorname{val}\left(\pi_{2}, G\right)$. Logo, $\operatorname{val}\left(\pi_{1}, G\right) \in \operatorname{val}\left(\tau_{2}, G\right)$, como queríamos.

A outra inclusão é análoga.

(ii) Reciprocamente, suponha que $\operatorname{val}\left(\tau_{1}, G\right)=\operatorname{val}\left(\tau_{2}, G\right)$. Precisamos mostrar que existe $p \in G$ tal que $p \Vdash^{*} \tau_{1}=\tau_{2}$. Considere $D$ o conjunto dos $r \in \mathbb{P}$ tais que

(I) $r \mathbb{H}^{*} \tau_{1}=\tau_{2}$ ou

(II) $\exists\left\langle\pi_{1}, s_{1}\right\rangle \in \tau_{1}\left(r \leq s_{1} \wedge \forall\left\langle\pi_{2}, s_{2}\right\rangle \in \tau_{2} \forall q \in \mathbb{P}\left(\left(q \leq s_{2} \wedge q \Vdash^{*} \pi_{1}=\pi_{2}\right) \rightarrow q \perp r\right)\right)$ ou

(III) $\exists\left\langle\pi_{2}, s_{2}\right\rangle \in \tau_{2}\left(r \leq s_{2} \wedge \forall\left\langle\pi_{1}, s_{1}\right\rangle \in \tau_{1} \forall q \in \mathbb{P}\left(\left(q \leq s_{1} \wedge q \Vdash^{*} \pi_{1}=\pi_{2}\right) \rightarrow q \perp r\right)\right)$.

\footnotetext{
${ }^{13}$ Lembramos que dissemos que $\in$ é um relação bem fundada. Isso significa que podemos pensar que os nomes estão numa hierarquia. Assim, assumimos que temos o resultado para fórmulas sobre nomes que estão abaixo, na hierarquia, dos nomes envolvidos na fórmula. Veja [Ku], Capítulo 3 .
} 
Primeiramente, note que se $r \in G$, então $r$ não satisfaz nem $(I I)$ nem $(I I I)$, pois suponha que satisfaça (II) (o outro caso é análogo), então existe $\left\langle\pi_{1}, s_{1}\right\rangle \in \tau_{1}$ como em $(I I)$. Daí, como $r \leq s_{1}$, temos que $s_{1} \in G$. Assim, $\operatorname{val}\left(\pi_{1}, G\right) \in \operatorname{val}\left(\tau_{1}, G\right)=\operatorname{val}\left(\tau_{2}, G\right)$. Fixe $\left\langle\pi_{2}, s_{2}\right\rangle \in \tau_{2}$, tal que $s_{2} \in G$ e $\operatorname{val}\left(\pi_{1}, G\right)=\operatorname{val}\left(\pi_{2}, G\right)$. Pela hipótese de indução, existe $q_{0} \in G$ tal que $q_{0} \Vdash^{*} \pi_{1}=\pi_{2}$. Assim, tomando $q \in G$ tal que $q \leq s_{2}, q_{0}$, temos que $q \Vdash^{*} \pi_{1}=\pi_{2}$, pelo Lema 4.36. Assim, por $(I I)$ temos que $q \perp r$, contradizendo que $q, r \in G$.

Assim, se $\neg \exists r \in G\left(r \Vdash^{*} \tau_{1}=\tau_{2}\right)$, então teremos que $D \cap G=\emptyset$. Se provarmos que $D$ é denso em $\mathbb{P}$, como $D \in M$, não pode acontecer que $D \cap G=\emptyset$ e daí, existe $r \in G$ tal que $r \Vdash^{*} \tau_{1}=\tau_{2}$, como queremos.

Seja $p \in \mathbb{P}$. Temos que ou $p \Vdash^{*} \tau_{1}=\tau_{2}$, ou pelo menos uma das condições 4.33(a)(i) e 4.33(a)(ii) falha. Suponha que 4.33(a)(i) falha (o caso em que 4.33(a)(ii) falha é análogo). Então, existe $\left\langle\pi_{1}, s_{1}\right\rangle \in \tau_{1}$ e $r \leq p$ tais que

$$
\forall q \leq r\left(q \leq s_{1} \wedge \forall\left\langle\pi_{2}, s_{2}\right\rangle \in \tau_{2}\left(\neg\left(q \leq s_{2} \wedge q \Vdash^{*} \pi_{1}=\pi_{2}\right)\right)\right) .
$$

Em particular, temos que $r \leq s_{1}$. Seja $\left\langle\pi_{2}, s_{2}\right\rangle \in \tau_{2}$. Se $q \leq s_{2}$ e $q \mathbb{H}^{*} \pi_{1}=\pi_{2}$, então temos que $q \perp r$, pois se eles tivessem uma extensão em comum, ela iria contradizer (4.1). Assim, $r \leq p$ e $r \in D$, pois $r$ satisfaz $(I I)$. Portanto, $D$ é denso e temos (ii) para fórmulas da forma $\tau_{1}=\tau_{2}$.

Deixamos as fórmulas da forma $\tau_{1}=\tau_{2}$ (que é análogo ao caso anterior). Suponhamos que já temos o resultado para $\phi$ atômica e provemos o resultado para as outras formas de $\phi$, por indução em sua complexidade. Para facilitar a notação, no que se segue não escreveremos $\tau_{1}, \ldots, \tau_{n}$ (mas estes podem facilmente ser escritos posteriormente).

Suponha $\phi$ da forma $\neg \varphi$ e que temos o resultado para $\varphi$ :

(i) Suponha $p \in G$ e $\left(p \Vdash^{*} \neg \varphi\right)^{M}$. Precisamos mostrar que $(\neg \varphi)^{M[G]}$. Suponha que não, então vale $(\varphi)^{M[G]}$. Pela hipótese indutiva, existe $q \in G$ tal que $\left(q \mathbb{1}^{*} \varphi\right)^{M}$. Seja $r \in G$ tal que $r \leq p$ e $r \leq q$. Então $\left(r \Vdash^{*} \varphi\right)^{M}$, contradizendo que $\left(p \Vdash^{*} \neq \varphi\right)^{M}$.

(ii) Suponha $(\neg \varphi)^{M[G]}$ e seja

$$
D=\left\{p \in \mathbb{P}:\left(p \Vdash^{*} \varphi\right)^{M} \vee\left(p \Vdash^{*} \neg \varphi\right)^{M}\right\}
$$


Temos que $D \in M$ e que $D$ é denso pela definição de $\mathbb{H}^{*}$. Assim, existe $p \in G \cap D$. Se $\left(p \Vdash^{*} \neg \varphi\right)^{M}$, acabou. Se não, temos que $\left(p \Vdash^{*} \varphi\right)^{M}$, e, pela hipótese indutiva, temos que $(\phi)^{M[G]}$, um absurdo.

Deixamos as fórmulas da forma $\varphi \wedge \psi$.

Suponha $\phi$ da forma $\exists x \varphi(x)$ e que temos o resultado para $\varphi$ : (i) Suponha $p \in G$ e $\left(p \Vdash^{*} \varphi(\sigma)\right)^{M}$. Então

$$
\left\{r \in \mathbb{P}: \exists \sigma \in M^{\mathbb{P}}\left(r \Vdash^{*} \varphi(\sigma)\right)^{M}\right\}
$$

é denso abaixo de $p$ e pertence a $M$. Então seja $r \in G$ tal que $\left(r \Vdash^{*} \varphi(\sigma)\right)^{M}$. Daí, pela hipótese indutiva, vale $(\varphi(\operatorname{val}(\sigma, G)))^{M[G]} \mathrm{e}$, portanto, vale $(\exists x \varphi(x))^{M[G]}$.

(ii) Suponha que vale $(\exists x \varphi(x))^{M[G]}$. Assim, seja $\sigma \in M^{\mathbb{P}}$ tal que $(\varphi(\operatorname{val}(\sigma, G)))^{M[G]}$. Então, pela hipótese de indução, existe $p \in G$ tal que $\left(p \mathbb{\Vdash}^{*} \varphi(\sigma)\right)^{M}$. Logo, temos que $\forall q \leq p\left(q \Vdash^{*} \varphi(\sigma)\right)^{M}$. Portanto, $\left(p \Vdash^{*} \exists x \varphi(x)\right)^{M}$.

O resultado acima é bastante técnico mas é quem garante a equivalência das relações $\Vdash$ e $\Vdash^{*}$. Vejamos então que, de fato, isto ocorre:

Lema 4.38. Sejam $M$ um modelo transitivo enumerável para $Z F C$ e $\mathbb{P}$ um forcing em M. Sejam $\phi\left(x_{1}, \ldots, x_{n}\right)$ uma fórmula e $\tau_{1}, \ldots, \tau_{n} \in M^{\mathbb{P}}$.

Então, para todo $p \in \mathbb{P}$ temos

$$
p \Vdash \phi\left(\tau_{1}, \ldots, \tau_{n}\right) \Leftrightarrow\left(p \Vdash^{*} \phi\left(\tau_{1}, \ldots, \tau_{n}\right)\right)^{M}
$$

Demonstração. $(\Leftarrow)$ : Imediato a partir do lema anterior $(i)$ e da definição de $\Vdash$.

$(\Rightarrow)$ : Suponha $p \in \mathbb{P}$ tal que $p \Vdash \phi\left(\tau_{1}, \ldots, \tau_{n}\right)$. Para mostrar que $\left(p \Vdash^{*} \phi\left(\tau_{1}, \ldots, \tau_{n}\right)\right.$ é suficiente mostrar (por 4.36) que $D=\left\{r \in \mathbb{P}:\left(r \mathbb{R}^{*} \phi\left(\tau_{1}, \ldots, \tau_{n}\right)\right)^{M}\right\}$ é denso abaixo de $p$. Suponha que não, então seja $q \leq p$ tal que $\neg \exists r \leq q, r \in D$. Assim, pela definição de $\mathbb{1}^{*}$, temos que $\left(q \Vdash^{*} \neg \phi\left(\tau_{1}, \ldots, \tau_{n}\right)\right)^{M}$. Daí, aplicando $(\Leftarrow)$, temos que $q \Vdash \neg \phi\left(\tau_{1}, \ldots, \tau_{n}\right)$. Seja $G$ um $\mathbb{P}$-genérico sobre $M$ tal que $q \in G$. Então, $\left(\neg \phi\left(\operatorname{val}\left(\tau_{1}, G\right), \ldots, \operatorname{val}\left(\tau_{n}, G\right)\right)\right)^{M[G]}$. Note que $p \in G$, já que $q \leq p$. Assim, temos que $\left(\phi\left(\operatorname{val}\left(\tau_{1}, G\right), \ldots, \operatorname{val}\left(\tau_{n}, G\right)\right)\right)^{M[G]}$. Absurdo.

Temos assim a equivalência das duas relações definidas. O motivo pelo qual isto acontece é que ao definir $\Vdash$ que era o que queríamos, analisamos suas propriedades, a fim 
de entender quais eram as propriedades de $\mathbb{P}$ que garantiam isso. Usando tais observações, definimos a relação $\mathbb{H}^{*}$ que, cumpriu, de fato, seu papel de ser definida dentro de $M$ e, ao mesmo tempo, ser equivalente a $\Vdash$. Assim, como $\Vdash$ "controla" a verdade em $M[G]$ e $\Vdash^{*}$ foi definida dentro de $M$, podemos controlar, a partir de $M$, a verdade em $M[G]$.

Por fim, usando esta equivalência, conseguimos o resultado central do forcing, que nos permite estudar as propriedades de $\mathbb{P}$ para obter informações sobre a verdade em $M[G]$ :

Teorema 4.39. Sejam $M$ um modelo transitivo enumerável para $Z F C$ e $\mathbb{P}$ um forcing em M. Sejam $\phi\left(x_{1}, \ldots, x_{n}\right)$ uma fórmula e $\tau_{1}, \ldots, \tau_{n} \in M^{\mathbb{P}}$.

Então, para todo $\mathbb{P}$-genérico $G$ sobre $M$ temos:

$$
\phi\left(\operatorname{val}\left(\tau_{1}, G\right), \ldots, \operatorname{val}\left(\tau_{n}, G\right)\right)^{M[G]} \Leftrightarrow \exists p \in G\left(p \Vdash \phi\left(\tau_{1}, \ldots, \tau_{n}\right)\right) .
$$

Demonstração. $(\Rightarrow)$ : Imediato a partir do resultado anterior e de 4.37(ii) que diz a mesma coisa sobre $\mathbb{1}^{*}$.

$(\Leftarrow)$ : Imediato a partir da definição de $\Vdash$.

Note que, neste momento, estamos prontos para usar o forcing: seja $\Phi$ uma fórmula, cuja consistência relativa com ZFC queremos provar. Suponha que $M$ é um modelo transitivo e enumerável para ZFC e $\mathbb{P}$ um forcing em $M$ e que existe $p \in \mathbb{P}$ tal que $p \Vdash \Phi$. Vimos que é possível então obter $G$ um $\mathbb{P}$-genérico sobre $M$ tal que $p \in G$. Assim, teremos que vale $\Phi^{M[G]}$. Além disso, sabemos que $M[G]$ é um modelo transitivo e enumerável para ZFC e que $M \subseteq M[G]$. Temos, portanto, as hipóteses do Princípio de Forcing e daí, segue que $\Phi$ é relativamente consistente com ZFC, como queríamos.

Retomando, para provar que uma fórmula $\Phi$ é relativamente consistente com ZFC, basta obter $\mathbb{P}$ um forcing e um elemento $p \in \mathbb{P}$ tal que $p \Vdash \Phi$. O corolário abaixo facilita o trabalho que teremos para achar um tal $p \in G$, quando a fórmula $\Phi$ é da forma $\neg \Phi_{1}$, ou $\exists x \Phi_{1}(x)$ :

Corolário 4.40. Sejam $M$ um modelo transitivo enumerável para $Z F C$ e $\mathbb{P}$ uma ordem parcial em $M$. Sejam $\phi\left(x_{1}, \ldots, x_{n}\right)$ uma fórmula e $\tau_{1}, \ldots, \tau_{n} \in M^{\mathbb{P}}$. Então:

(i) $\left\{p \in \mathbb{P}:\left(p \Vdash \phi\left(\tau_{1}, \ldots, \tau_{n}\right)\right) \vee\left(p \Vdash \neg \phi\left(\tau_{1}, \ldots, \tau_{n}\right)\right\}\right.$ é denso. 
(ii) $p \Vdash \neg \phi\left(\tau_{1}, \ldots, \tau_{n}\right)$ se, e somente se, $\neg \exists q \leq p\left(q \Vdash \phi\left(\tau_{1}, \ldots, \tau_{n}\right)\right)$.

(iii) $p \Vdash \exists x \phi\left(x, \tau_{1}, \ldots, \tau_{n}\right)$ se, e somente se, $\left\{r \leq p: \exists \sigma \in M^{\mathbb{P}}\left(r \Vdash \phi\left(\sigma, \tau_{1}, \ldots, \tau_{n}\right)\right)\right\}$ é denso abaixo de $p$.

(iv) Se $p \Vdash \exists x\left(x \in \sigma \wedge \phi\left(x, \tau_{1}, \ldots, \tau_{n}\right)\right)$, então $\exists q \leq p \exists \pi \in \operatorname{dom}(\sigma)\left(q \Vdash \phi\left(\pi, \tau_{1}, \ldots, \tau_{n}\right)\right)$.

Demonstração. Temos que $(i),(i i)$ e $(i i i)$ valem para $\Vdash^{*}$ e, portanto, valem para $\Vdash$ por 4.38 .

Para (iv) temos que, suponha que $p \Vdash \exists x\left(x \in \sigma \wedge \phi\left(x, \tau_{1}, \ldots, \tau_{n}\right)\right)$. Então seja $G$ $\mathbb{P}$-genérico tal que $p \in G$. Pela definição de $\Vdash$, existe $a \in M[G]$ tal que $a \in \operatorname{val}(\sigma, G)$ e $\left(\phi\left(a, \operatorname{val}\left(\tau_{1}, G\right), \ldots, \operatorname{val}\left(\tau_{n}, G\right)\right)^{M[G]}\right.$. Temos que $a=\operatorname{val}(\pi, G)$ para algum $\pi \in \sigma$, então, por 4.39 temos que existe $r \in G$ tal que $r \Vdash \phi\left(\pi, \tau_{1}, \ldots \tau_{n}\right)$. Tomando $q \in G \operatorname{com} q \leq p$ e $q \leq r$, temos $q \Vdash \phi\left(\pi, \tau_{1}, \ldots, \tau_{n}\right)$.

Mais uma propriedade interessante do forcing é que podemos decidir, abaixo de um $p \in \mathbb{P}$, que ou $\Phi$ ou $\neg \Phi$ é forçada:

Proposição 4.41. Seja $\mathbb{P}$ um forcing e $\Phi$ uma fórmula. Então, existe $q \leq p$ tal que $q \Vdash \Phi$ ou $q \Vdash \neg \Phi$.

Demonstração. Basta notar que

$$
D=\left\{q \in \mathbb{P}: q \Vdash^{*} \Phi \text { ou } q \Vdash^{*}\right\}
$$

é denso em $\mathbb{P}$. Isto segue da definição de $\mathbb{1}^{*}$.

Não usaremos mais, no que segue, a notação $\mathbb{}^{*}$. Tendo a equivalência das duas relações, não há problema em usar um símbolo apenas para ambas as relações.

Vejamos ainda mais um resultado, consequência da equivalência destas relações e que nos será útil ao trabalharmos com o forcing de Sacks, na Seção 4.7.

Proposição 4.42. Sejam $s, s_{1}, \ldots, s_{n} \in \mathbb{S}$ e sejam $\Phi_{1}, \ldots, \Phi_{n}$ fórmulas. Se $\left\{s_{1}, \ldots, s_{n}\right\}$ forma uma anticadeia maximal abaixo de $s$ e para cada $1 \leq i \leq n$ temos que $s_{i} \Vdash \Phi_{i}$, então $s \Vdash \Phi_{1} \vee \cdots \vee \Phi_{n}$. Neste caso, se existe uma fórmula $\Phi$ tal que para todo $1 \leq i \leq n$ tem-se que $\Phi_{i}=\Phi$, então $s \Vdash \Phi$. 
Demonstração. Suponha $s, s_{1}, \ldots, s_{n} \in \mathbb{S}$ e $\Phi_{1}, \ldots, \Phi_{n}$ fórmulas tais que $\left\{s_{1}, \ldots, s_{n}\right\}$ forma uma anticadeia maximal abaixo de $s$ e para cada $1 \leq i \leq n$ temos que $s_{i} \Vdash \Phi_{i}$.

Daí, para cada $s^{\prime} \leq s$, temos que existe $1 \leq i \leq n$ tal que $s_{i}$ é compatível com $s^{\prime}$. Logo, existe $t \leq s^{\prime}, s_{i}$. Como $s_{i} \Vdash \Phi$, segue que $t \Vdash \Phi_{i}$. Assim,

$$
\left\{t \in \mathbb{P}: \exists 1 \leq i \leq n, t \Vdash \Phi_{i}\right\}=\left\{t \in \mathbb{P}: t \Vdash \Phi_{1} \vee \cdots \vee \Phi_{n}\right\}
$$

é denso abaixo de $s$ e, portanto, $s \Vdash \Phi_{1} \vee \cdots \vee \Phi_{n}$.

A segunda parte do resultado segue diretamente da primeira e do fato que $\Phi \vee \cdots \vee \Phi=$ $\Phi$.

Este resultado é interessante quando trabalhamos com forcing's que nos dão algumas anticadeias maximais abaixo de um $p$, uma vez que neste caso, se sabemos que os elementos desta anticadeia forçam algumas fórmulas, então este elemento $p$ força a alternativa destas coisas. Veremos na Seção 4.7 que o forcing de Sacks possui boas propriedades neste sentido.

\subsection{Preservação de cardinais}

O objetivo desta seção é apresentar alguns resultados sobre preservação de cardinais. Como já dissemos, o forcing foi criado por Cohen para provar a consistência da negação da hipótese do contínuo (CH). Tal hipótese afirma que $\omega_{1}$, o primeiro cardinal não enumerável é igual ao cardinal do contínuo $2^{\omega}$ (a cardinalidade do conjunto dos números reais). Esta hipótese tem profundas consequências em todas as áreas da matemática.

Porém, não é nosso interesse aqui seguir os passos de Cohen e mostrar a consistência da negação de CH. Mas porque então queremos resultados sobre preservação de cardinais? Em primeiro lugar, para entender um pouco melhor como se comportam objetos quando passamos do modelo inicial à extensão. Temos, por exemplo, que se $\alpha$ é um ordinal no modelo inicial, então $\alpha$ será um ordinal em toda sua extensão, pois "ser um ordinal" é absoluto para modelos transitivos. Porém, com os cardinais não acontece o mesmo: "ser um cardinal" não é absoluto. Assim, temos que um cardinal $\kappa \in M$ será um ordinal em $M[G]$ que pode ou não ser um cardinal. Mas podemos precisar saber a cardinalidade de algum objeto do modelo inicial na extensão, sabendo sua cardinalidade no modelo inicial. 
Para calculá-la, os resultados apresentados nesta seção serão necessários. Para entender isto, vejamos a seguinte definição:

Definição 4.43. Seja $\mathbb{P}$ um forcing. Dizemos que $\mathbb{P}$ preserva cardinais se para todo $M$ modelo transitivo enumerável tal que $\mathbb{P} \in M$, todo cardinal $\kappa \in M$, e todo filtro genérico $G$ sobre $M$ tem-se que $\kappa$ é um cardinal em $M[G]$.

Os primeiros forcing's que consideraremos, que preservam cardinais são os forcing's com a propriedade de c.c.c.:

Definição 4.44. Seja $\kappa$ um cardinal. Dizemos que um forcing $\mathbb{P} e ́ \kappa$-c.c. se toda anticadeia em $\mathbb{P}$ tem cardinalidade menor que $\kappa$. Dizemos que $\mathbb{P}$ é c.c.c. se é $\omega_{1}$-c.c.

Proposição 4.45. Se $\mathbb{P}$ é um forcing c.c.c., então $\mathbb{P}$ preserva cardinais.

Demonstração. Indicamos [Ku], Teorema 5.10, página 207.

Outros forcing's que preservam cardinais são forcing's com a propriedade de $\kappa$-c.c. e $\sigma$-fechados:

Definição 4.46. Seja $\kappa$ um cardinal. Dizemos que um forcing $\mathbb{P}$ é $\kappa$-fechado se para toda cadeia $\left(p_{\alpha}\right)_{\alpha<\kappa} \subseteq \mathbb{P}$ decrescente, existe $p \in \mathbb{P}$ tal que $p \leq p_{\alpha}$ para todo $\alpha<\kappa$. Dizemos que um forcing $\mathbb{P}$ é $\sigma$-fechado se é $\omega_{1}$-fechado.

Proposição 4.47. Seja $\kappa$ um cardinal. Se $\mathbb{P}$ é um forcing $\kappa^{+}$-c.c. e $\kappa$-fechado, então $\mathbb{P}$ preserva cardinais.

Demonstração. Indicamos [Ku], Teorema 6.9, página 213 e Corolário 6.15, página 215.

Temos assim que um forcing com alguma destas boas propriedades preserva cardinais, como é o caso do forcing de Cohen.

\subsection{O forcing de Cohen}

Apresentamos, nesta seção, o forcing de Cohen. Ele não será aplicado no que segue, mas optamos por apresentá-lo, dada sua importância histórica. Lembramos que o forcing de 
Cohen foi criado por Cohen para mostrar a consistência da negação da hipótese do contínuo (CH), ou seja, de forma que tal hipótese não valesse em $M[G]$. A consistência desta afirmação já havia sido obtida, através de um modelo. Comecemos com sua definição:

Definição 4.48. Seja $\kappa$ um cardinal regular. Chamamos de forcing de Cohen o conjunto

$$
F n(\kappa, 2)=\{f: \operatorname{dom}(f) \rightarrow\{0,1\} \text { tais que } \operatorname{dom}(f) \subseteq \kappa \text { é finito. }\}
$$

com a ordem inversa à da extensão, isto é, se $f, g \in F n(\kappa, 2)$, então $f \leq g$ se $\operatorname{dom}(f) \supseteq$ $\operatorname{dom}(g)$ e $\left.f\right|_{\operatorname{dom}(g)}=g$.

Convém mencionar aqui que nosso primeiro exemplo de forcing(o Exemplo 4.10) é desta forma. Tendo sido criado para que não valesse $\mathrm{CH}$ no modelo $M[G]$ e assumindo que $\mathrm{CH}$ valia em $M$ (uma vez que a consistência de $\mathrm{CH}$ já estava provada), gostaríamos que este forcing preservasse cardinais, para que pudéssemos controlar a cardinalidade de $\wp(\mathbb{N})$ em $M[G]$, mas que acrescentasse novos conjuntos de números naturais ao modelo $M[G]$. Vejamos um primeiro resultado:

Proposição 4.49. $F n(\kappa, 2)$ tem c.c.c. e, portanto, preserva cardinais.

Demonstração. Indicamos [Ku], Lema 5.4, página 205.

Já temos assim que $F n(\kappa, 2)$ preserva cardinais e podemos controlar a cardinalidade de $\wp(\mathbb{N})$ em $M[G]$. Mas será que este forcing acrescenta novos conjuntos de números naturais? Vejamos:

Lema 4.50. Consideremos $\mathbb{P}=F n(\omega, 2)$. Seja $M$ um modelo transitivo enumerável para $Z F C$ e seja $G$ um filtro $\mathbb{P}$-genérico sobre $M$. Então $\cup G$ é uma função de $\omega$ sobre $\{0,1\}$.

Demonstração. Indicamos [Ku], Lema 5.2, página 205.

Notemos que podemos identificar a função obtida através deste lema $\cup G$ com o conjunto dos números naturais em que ela vale 1. Por outro lado, sabemos que $G \notin M$ e podemos garantir então que $\cup G \notin M$ e, portanto, nosso conjunto de naturais com o qual identificamos $\cup G$ também não está em $M$. Temos assim que acrescentamos pelo menos um conjunto de naturais a $M[G]$. Para garantir que temos muito mais conjuntos de naturais, trabalhamos com forcing da forma $F n(\kappa, 2)$ 
Proposição 4.51. Seja $M$ um modelo transitivo e enumerável para $Z F C$ e consideremos $\kappa$ um cardinal em $M$ e $\mathbb{P}=(F n(\kappa, 2))^{M} \in M$. Se $G$ é um filtro $\mathbb{P}$-genérico sobre $M$, então $M[G] \Vdash 2^{\omega} \geq|\check{\kappa}|$.

Demonstração. Indicamos [Ku], Lema 5.3, página 205.

Combinando o resultado acima com o fato que $F n(\kappa, 2)$ preserva cardinais, garantimos que $\mathrm{CH}$ não vale em $M[G]$ :

Teorema 4.52. Sejam $M$ um modelo transitivo enumerável para $Z F C+C H, \kappa \in M$ um cardinal regular e $G$ um $F n(\kappa, 2)$-genérico sobre $M$. Então, temos $M[G] \vDash 2^{\omega}=\kappa$.

Demonstração. Indicamos [Ku], Lema 5.14, página 209.

Para ilustrar que propriedades este novo conjunto de naturais pode possuir, temos o seguinte:

Lema 4.53. Seja $M$ um modelo de ZFC e $G$ um $F n(\omega, 2)$-genérico sobre $M$. Suponha c um real de Cohen sobre $M$, isto é, $c \in \wp(\mathbb{N}) \cap M[G]$ e $c \notin M$. Então c é independente ${ }^{14}$ de todo subconjunto infinito e coinfinito de $\mathbb{N}$ de $M$, isto é, se $X \in \wp(\mathbb{N}) \cap M$ é infinito, então $X \cap c, X \backslash c, c \backslash X e \mathbb{N} \backslash(c \cup X)$ são infinitos.

Demonstração. Seja $A \subseteq \omega$ infinito e coinfinito, $A \in M$ e seja $c=\bigcup G$ um real de Cohen sobre $M$. Considere os seguintes subconjuntos de $F n(\omega, 2)$ :

$$
D_{k}=\{p \in F n(\omega, 2): \text { existe } n>k \text { tal que } p(n)=0 \text { e } n \in A\}
$$

Para todo $k \in \omega, D_{k}$ é denso, pois dado $p \in F n(\omega, 2)$, como $A$ é infinito, existe $n \in A \backslash(\operatorname{dom}(p) \cup\{0, \ldots, k\})$. Definimos $q=p \cup(n, 0)$ e temos que $q \leq p$ e $q \in D_{k}$.

Assim, se $c$ é um real de Cohen sobre $M$, temos que existem infinitos $n \in A$ tais que $n \notin c$, isto é, $A \backslash c$ é infinito.

\footnotetext{
${ }^{14}$ Lembramos que, na realidade, $c$ é uma função de $\omega$ em 2. Porém, podemos identificar tal função com o conjunto dos números naturais em que ela vale 1. Reciprocamente, se temos um conjunto de naturais, a função característica deste conjunto é uma função de $\omega$ em 2.
} 
Considerando para cada $k \in \omega$,

$$
E_{k}=\{p \in F n(\omega, 2): \text { existe } n>k \text { tal que } p(n)=1 \text { e } n \in A\}
$$

como $A$ é infinito, temos que cada $E_{k}$ é denso e assim existem infinitos $n \in A$ tais que $n \in c$, isto é, $A \cap c$ é infinito.

Analogamente, como $N \backslash A$ é infinito, temos que $(N \backslash A) \backslash c$ e $(N \backslash A) \cap c$ são infinitos.

Logo, $c$ é independente de $A$.

Por fim, um último resultado também para ilustrar de que forma este novo conjunto de naturais influencia as propriedades de $(\wp(\mathbb{N}))^{M}$.

Corolário 4.54. Se $M$ é um modelo de ZFC e c é um real de Cohen sobre $M$, então, em $M[c](M[c]$ é $M[G]$, onde $c=\bigcup G)$ não existe um ultrafiltro não principal em $(\wp(\mathbb{N}))^{M[c]}$ gerado por elementos de $M$.

Demonstração. Suponha que $u \in M[c]$ é um ultrafiltro não principal gerado por elementos de $M$. Como $u$ é ultrafiltro em $M[c]$, temos que $c \in u$ ou $N \backslash c \in u$. Se $c \in u$, como $u$ é gerado por elementos de $M$, existe $A \in M, A \subseteq N$ infinito tal que $A \subseteq c$, que contradiz o lema anterior. Se $c \notin u$, então, $N \backslash c \in u$ e daí existe $A \in M, A \subseteq N$ infinito tal que $A \subseteq N \backslash c$, ou seja, $A \cap c=\emptyset$, que contradiz o lema anterior.

\subsection{O forcing de Sacks}

O objetivo desta última seção é apresentar o forcing de Sacks. Tal forcing será utilizado no Capítulo 7, para mostrar a consistência de uma afirmação sobre a densidade de espaços de Grothendieck da forma $C(K)$. Chamamos de forcing de Sacks um forcing específico que garante que temos um real a mais que em $M$, no modelo $M[G]$. O forcing que usaremos no Capítulo 7 é, na realidade, um produto de tais forcing's, que garante que temos muitos reais a mais no modelo $M[G]$ que em $M$. 


\subsubsection{O forcing de Sacks simples}

Vejamos então um pouco sobre o forcing de Sacks simples, que garante apenas que temos um real a mais em $M[G]$ que no modelo inicial. Comecemos com sua definição:

Definição 4.55. Seja $1_{\mathbb{S}}=\bigcup\left\{\{0,1\}^{n}: n \in \omega\right\}$. Dizemos que um subconjunto não vazio $s \subseteq 1_{\mathbb{S}}$ é uma árvore perfeita $s e$

1. $\left.\forall p \in s \quad \forall n \quad p\right|_{n} \in s$;

2. $\forall p \in s \quad \exists q, r \in s \quad p \subseteq q, r$ e q e r são incomparáveis.

A condição (1) diz que $s$ é, de fato, uma árvore. A condição (2) pode ser interpretada como "uma árvore perfeita bifurca acima de todo nó" .

Definição 4.56. Chamamos de forcing de Sacks (e denotamos por $\mathbb{S}$ ) o conjunto de todas as árvores perfeitas com a ordem da inclusão. Além disso, dados $s \in \mathbb{S}$ e $p \in s$, definimos o nível de bifurcação $p$ em $s$ (denotamos por bif $(p, s)$ ) como a cardinalidade de $\left\{i<|p|: \exists q \in s,|q|>i,\left.q\right|_{i}=\left.p\right|_{i}\right.$ e $\left.\left.q\right|_{i+1} \neq\left. p\right|_{i+1}\right\}$. Este é o número de vezes que $s$ bifurca abaixo de $p$. Dado $n \in \mathbb{N}$, definimos o $n$-ésimo nível de bifurcação de $s$ (denotamos por $l(n, s)$ ) como o conjunto dos elementos de s com nivel de bifurcação $n$. Note que $|l(n, s)|=2^{n}$. Além disso, dizemos que $s \leq_{n} t$ se $s \leq t$ e $l(n, s)=l(n, t)$.

Lema 4.57 (de fusão). Seja $s_{n} \in \mathbb{S}$ tais que $s_{n+1} \leq_{n} s_{n}$. Então, existe $s \in \mathbb{S}$ tal que $\forall n \in \omega, s \leq s_{n}$.

Demonstração. Dado $n \in \mathbb{N}$, defina $\overline{l\left(s_{n}, n\right)}=\left\{p \in 1_{\mathbb{S}}: \exists q \in l\left(s_{n}, n\right), p \leq q\right\}$ e chamamos a este conjunto de o fecho para baixo de $l\left(s_{n}, n\right)$. Considere $s=\bigcup_{n \in \mathbb{N}} \overline{l\left(s_{n}, n\right)}$. Vejamos que $s \in \mathbb{S}$ e $s \leq s_{n}$ para todo $n \in \mathbb{N}$.

É claro que $s$ é um subconjunto não vazio de $1_{\mathbb{S}}$. Para verificar a condição 1 da Definição 4.55 , seja $p \in s$ e $q \in 1_{\mathbb{S}}$ tal que $q \leq p$. Considere $n \in \mathbb{N}$ tal que $p \in \overline{l\left(s_{n}, n\right)}$ e temos que $q \in \overline{l\left(s_{n}, n\right)} \subseteq s$. Para a condição 2, dado $p \in s$, considere $n \in \mathbb{N}$ tal que $p \in \overline{l\left(s_{n}, n\right)} \subseteq s_{n+1}$. Sejam $q_{1}, q_{2} \in l\left(s_{n+1}, n+1\right)$ tais que $p \subseteq q_{1}, q_{2}$. Como $q_{1}, q_{2} \in s$, temos a propriedade.

Por fim, para mostrar que $s \leq s_{n}$ para todo $n \in \mathbb{N}$, considere $p \in s$ e $n \in \mathbb{N}$ tal que $p \in \overline{l\left(s_{n}, n\right)}$. Se $m \geq n$, temos que $p \in s_{n} \subseteq s_{m}$. Se $m<n$, então $l\left(s_{n}, n\right)=l\left(s_{m}, n\right)$ e daí, $\overline{l\left(s_{n}, n\right)}=\overline{l\left(s_{m}, n\right)}$ e assim, $p \in \overline{l\left(s_{m}, n\right)} \subseteq s_{m}$. 
Definição 4.58. Dado $s \in \mathbb{S}$ e $p \in s$, definimos $s \mid p \in \mathbb{S}$ por

$$
s \mid p=\{q \in s: q \subseteq p \text { ou } p \subseteq q\}
$$

Lema 4.59. $\{s \mid p: p \in l(s, n)\}$ é uma anticadeia maximal abaixo de $s$ de cardinalidade $2^{n}$.

Demonstração. Para mostrar que é anticadeia, notemos que se $p_{1}, p_{2} \in l(s, n)$ são tais que $p_{1} \neq p_{2}$, então $s\left|p_{1} \cap s\right| p_{2}$ é finito e, portanto, são incompatíveis. De fato, se $t \in s\left|p_{1} \cap s\right| p_{2}$, então $t \subseteq p_{1}, p_{2}$, pois $p_{1} \nsubseteq p_{2}$ e $p_{2} \nsubseteq p_{1}$.

Para mostrar que é maximal abaixo de $s$, tomemos $r \leq s$ e $p \in r$ tal que $b i f(p, r)=n$. Assim, bif $(p, s) \geq n$. Tomemos $q \subseteq p, q \in l(s, n)$. Daí, $r|p \leq s| q, r$. Logo, $s \mid q$ e $r$ são compatíveis. Logo, temos que o conjunto forma uma anticadeia maximal abaixo de $s$.

Notemos que o lema acima se torna bastante poderoso, quando o associamos à Proposição 4.42 .

Lema 4.60. Seja $s \in \mathbb{S}$ e $n \in \mathbb{N}$. Se para cada $p \in l(s, n)$ temos $r(p) \in \mathbb{S}$ tal que $r(p) \leq s \mid p$, então existe $r \leq_{n}$ s tal que $r \mid p \leq r(p)$ para todo $p \in l(s, n)$.

Demonstração. Suponha $s, n, r(p)$ como na hipótese. Tomemos $r=\bigcup\{r(p): p \in l(s, n)\}$. Como cada $r(p) \leq s \mid p \leq s$, temos que $r \leq s$. Além disso, é fácil ver que $l(r, n)=l(s, n)$ e $r \mid p \leq r(p)$.

Para ilustrar um pouco as diferenças entre o forcing de Cohen e o forcing de Sacks, temos o seguinte:

Proposição 4.61. Seja $M$ um modelo transitivo e enumerável para $Z F C, \mathbb{P}=(\mathbb{S})^{M} \in M$ e G um $\mathbb{P}$-genérico sobre $M$. Se $X$ é um subconjunto dos naturais infinito e coinfinito de $M[G]$, então existe $Y$ um subconjunto dos naturais infinito de $M$ tal que

$$
M[G] \vDash \check{Y} \subseteq X \text { ou } \check{Y} \subseteq \mathbb{N} \backslash X \text {. }
$$

Demonstração. Seja $s \in \mathbb{S}$ e seja $\dot{X}$ um $\mathbb{S}$-nome para $X$.

Se existe $t \leq s$ e $Y \subseteq \mathbb{N}$ infinito tal que $t \Vdash \check{Y} \subseteq \check{\mathbb{N}} \backslash \dot{X}$, então não há o que provar. Senão, construímos, por indução, seqüências $\left(s_{n}\right)_{n \in \mathbb{N}} \subseteq \mathbb{S}$ e $\left(k_{n}\right)_{n \in \mathbb{N}} \subseteq \mathbb{N}$ tais que 
- $s_{n+1} \leq_{n} s_{n}$

- $k_{n}<k_{n+1}$

- $s_{n} \Vdash \check{k}_{n} \in \dot{X}$.

Daí, pelo Lema de Fusão (4.57), existe $s^{*} \leq s_{n}$ para todo $n \in \mathbb{N}$. Tal $s^{*}$ é tal que se $Y=\left\{k_{n}: n \in \mathbb{N}\right\}$, então $s^{*} \Vdash \check{Y} \subseteq \dot{x}$ e temos o resultado.

Para a construção, suponha que temos $p_{0}, \ldots, p_{n}$ e $k_{0}, \ldots, k_{n}$ como queremos. Para todo $p \in l\left(s_{n}, n\right)$, temos que $s_{n} \mid p \leq s$ e não satisfaz o Caso 1 . Assim, para todo $p \in l\left(s_{n}, n\right)$, temos que

$$
A_{p}=\left\{k \in \mathbb{N}: k \geq k_{n}, s_{n} \mid p \Vdash \check{k} \in \check{\mathbb{N}} \backslash \dot{X}\right\}
$$

é finito, pois $s_{n} \mid p \Vdash \check{A}_{p} \subseteq \check{\mathbb{N}} \backslash \dot{X}$. Seja então $k_{n+1}>k_{n}$ tal que $k_{n+1} \notin \bigcup_{p \in l\left(s_{n}, n\right)} A_{p}$.

Como $s_{n} \mid p \Downarrow \check{k}_{n+1} \in \dot{X}$, existem $q(p) \leq s_{n} \mid p$ tais que $q(p) \Vdash \check{k}_{n+1} \in \dot{X}$. Tomemos então $s_{n+1} \leq_{n} s_{n}$ tal que $s_{n+1} \mid p \leq q(p)$, como no lema anterior. Assim, $s_{n+1} \Vdash \check{k}_{n+1} \in \dot{X}$, pelo Lema 4.42 .

Comparando a proposição acima com o Lema 4.53, temos uma grande diferença entre os forcing's de Cohen e de Sacks.

\subsubsection{O produto de forcing's de Sacks}

Como dissemos, se forçamos com $\mathbb{S}$, obtemos um modelo que possui um real a mais que o modelo inicial. Portanto, para obter muitos reais a mais (por exemplo $2^{\omega}=\omega_{2}$ ), precisamos forçar com a seguinte ordem:

Definição 4.62. Dado um cardinal regular $\kappa$ e $s: \kappa \rightarrow \mathbb{S}$, chamamos de suporte de $s$ (e denotamos por $\operatorname{supp}(s))$ o conjunto $\left\{\alpha \in \kappa: s(\alpha) \neq 1_{\mathbb{S}}\right\}$. Dai, $\mathbb{S}^{\kappa}$ é o conjunto das funções $s: \kappa \rightarrow \mathbb{S}$ com $\operatorname{supp}(s)$ enumerável, com a seguinte ordem: $s \leq t$ se $\operatorname{supp}(s) \subseteq \operatorname{supp}(t) e$ para todo $\alpha \in \operatorname{supp}(s), s(\alpha) \subseteq t(\alpha)$. Dados $s \in \mathbb{S}^{\kappa}, F \subseteq \operatorname{supp}(s)$ e $n \in \mathbb{N}$, definimos

$$
l(F, n, s)=\{\sigma: \operatorname{supp}(\sigma)=F \text { e para todo } \alpha \in F, \sigma(\alpha) \in l(n, s(\alpha))\}
$$

Além disso, dizemos que $s \leq_{F, n} t$ se $s \leq t$ e para todo $\alpha \in F, s(\alpha) \leq_{n} t(\alpha)$. 
Sabe-se que $\mathbb{S}^{\kappa} \Vdash \omega_{1} \leq c=\kappa$.

Lema 4.63 (de fusão). Sejam $s_{n} \in \mathbb{S}^{\kappa}, F_{n} \subseteq \kappa$ finitos tais que

- $F_{n} \subseteq F_{n+1}$;

- $\bigcup\left\{F_{n}: n \in \mathbb{N}\right\}=\bigcup\left\{\operatorname{supp}\left(s_{n}\right): n \in \mathbb{N}\right\}$

- $s_{n+1} \leq_{F_{n}, n} s_{n}$.

Então, existe $s \in \mathbb{S}^{\kappa}$ tal que para todo $n \in \mathbb{N}, s \leq s_{n}$.

Demonstração. Para cada $n \in \mathbb{N}$, fixemos $\left(\alpha_{i}^{n}\right)_{i \in \mathbb{N}}$ uma enumeração para $\operatorname{supp}\left(s_{n}\right)$. Tomemos a cada passo, $F_{n}=\left\{\alpha_{i}^{j}: i, j \leq n\right\}$ e teremos 1 e 2 .

Seja $\xi \in \kappa$. Se $\xi \notin \bigcup_{n \in \mathbb{N}} \operatorname{supp}\left(s_{n}\right)$, então $s_{n}(\xi)=1_{\xi}$ para todo $n \in \mathbb{N}$. Tome $s(\xi)=1$ e temos $s(\xi) \leq s_{n}(\xi)$ para todo $n \in \mathbb{N}$.

Senão, existe $N \in \mathbb{N}$ tal que $\xi \in F_{n}$ para todo $n \geq N$. Como $s_{n+1} \leq_{F_{n}, n} s_{n}$, temos que para todo $n \geq N, s_{n+1}(\xi) \leq_{n} s_{n}(\xi)$. Daí, pelo Lema 4.57, existe $s(\xi) \leq s_{n}(\xi)$ para todo $n \in \mathbb{N}$.

Assim, $s=(s(\xi))_{\xi \in \kappa} \in \mathbb{S}^{\kappa}$, pois $\left|\bigcup_{n \in \mathbb{N}} \operatorname{supp}\left(s_{n}\right)\right| \leq \omega$ e $s \leq s_{n}$ para todo $n \in \mathbb{N}$, pela construção de $s$.

Definição 4.64. Dados $s \in \mathbb{S}^{\kappa}, F \subseteq \kappa$ finito e $\sigma: F \rightarrow 1_{\mathbb{S}}$, definimos $s \mid \sigma \in \mathbb{S}^{\kappa}$ por $(s \mid \sigma)(\xi)=s(\xi) \mid \sigma(\xi)$ se $\xi \in F$ e $(s \mid \sigma)(\xi)=s(\xi)$, se $\xi \notin F$.

Lema 4.65. $\{s \mid \sigma: \sigma \in l(F, n, s)\}$ é uma anticadeia maximal abaixo de $s$ de cardinalidade $2^{n|F|}$.

Demonstração. Para mostrar que é anticadeia, notemos que se $\sigma, \tau \in l(F, n, s)$ são tais que $\sigma \neq \tau$, então existe $\xi \in F$ tais que $\sigma(\xi) \neq \tau(\xi)$. Por definição, $(s \mid \sigma)(\xi)=s(\xi) \mid \sigma(\xi)$ e $(s \mid \tau)(\xi)=s(\xi) \mid \tau(\xi)$. Como $\sigma(\xi), \tau(\xi) \in l(s(\xi), n)$, pelo Lema $4.59, s(\xi) \mid \sigma(\xi)$ e $s(\xi) \mid \tau(\xi)$ são incompatíveis. Logo, é anticadeia.

Para mostrar que é maximal abaixo de $s$, tomemos $t \leq s$. Sabemos, do Lema 4.59 que para todo $\xi \in F,\{s(\xi) \mid \sigma: \sigma \in l(s, n)\}$ é uma anticadeia maximal abaixo de $s(\xi)$. Assim, para todo $\xi \in F$, existe $\sigma_{\xi} \in l(s(\xi), n)$ tal que $s(\xi) \mid \sigma_{\xi}$ é compatível com $t(\xi)$. Defina 
$\sigma(\xi)=\sigma_{\xi}$ e temos que $s \mid \sigma$ é compatível com $t$, pois se $\xi \in F,(s \mid \sigma)(\xi)=s(\xi)|\sigma(\xi)=s(\xi)| \sigma_{\xi}$ que é compatível com $q(\xi)$.

Notemos ainda mais uma vez a força que o lema acima ganha, quando o utilizamos junto com a Proposição 4.42 .

Lema 4.66. Sejam $s \in \mathbb{S}^{\kappa}, n \in \mathbb{N}$ e $F \subseteq \kappa$ finito. Se $\sigma \in l(F, n, s)$ e $t \in \mathbb{S}^{\kappa}$ é tal que $t \leq s \mid \sigma$, então existe $s^{*} \leq_{F, n} s$ tal que $s^{*} \mid \sigma \leq t$.

Demonstração. Sejam $s, n, F, \sigma, t$ como na hipótese. Fixemos $\xi \in F$. Como $\sigma \in l(F, n, s)$, temos que $\sigma(\xi) \in l(p(\xi), n)$. Além disso, $t \leq s \mid \sigma$ e, portanto, $t(\xi) \leq(s \mid \sigma)(\xi)=s(\xi) \mid \sigma(\xi)$.

Dado $\tau \in l(s(\xi), n), \tau \neq \sigma(\xi)$ tomemos $q(\tau)=s(\xi) \mid \tau \in \mathbb{S}$ e $q(\sigma(\xi))=t(\xi) \in \mathbb{S}$. Assim, para todo $p \in l(s(\xi), n), q(p) \leq s(\xi) \mid p$. Logo, pelo Lema 4.60 , existe $s^{*}(\xi) \leq_{n} s(\xi)$ tal que $s^{*}(\xi) \mid p \leq q(p)$ para todo $p \in l(s(\xi), n)$.

Se $\xi \notin F$, tome $s^{*}(\xi)=t(\xi)$. Temos que $s^{*}=\left(s^{*}(\xi)\right)_{\xi \in \kappa} \in \mathbb{S}^{\kappa}$. Para cada $\xi \in F$, temos que $s^{*}(\xi) \leq s(\xi)$. Mais ainda, se $\xi \in F,\left(s^{*} \mid \sigma\right)(\xi)=s^{*}(\xi) \mid \sigma(\xi) \leq t(\xi)$ e se $\xi \notin F$, $\left(s^{*} \mid \sigma\right)(\xi)=s^{*}(\xi)=t(\xi)$. Logo, $s^{*} \mid \sigma \leq t$.

Definição 4.67. Seja $\mathbb{P}$ um forcing. Dizemos que $M \subseteq \mathbb{P}$ é aberto se é fechado para baixo, isto é, se $s \in M$ e $t \leq s$, então $t \in M$.

Lema 4.68. Se $s \in \mathbb{S}^{\kappa}, F \subseteq \operatorname{supp}(s)$ é finito, $n \in \mathbb{N}$ e $D$ é um aberto denso abaixo de $s$ em $\mathbb{S}^{\kappa}$, então existe $s^{*} \in \mathbb{S}^{\kappa}$ tal que $s^{*} \leq_{F, n} s$ e para todo $\sigma \in l(F, n, s)$, tem-se que $s^{*} \mid \sigma \in D$.

Demonstração. Seja $K=2^{n|F|}$. Seja $l(F, n, s)=\left\{\sigma_{i}: 0 \leq i<K\right\}$.

Tomemos $t_{0} \leq s \mid \sigma_{0}$ tal que $t_{0} \in D$. Pelo lema anterior, existe $s_{0} \leq_{F, N} s$ tal que $s_{0} \mid \sigma_{0} \leq t_{0}$. Como $D$ é aberto denso e $t_{0} \in D$, então $s_{0} \in D$.

Achemos então $t_{1} \leq s_{0} \mid \sigma_{1}$ tal que $t_{1} \in D$ e tomemos, pelo lema anterior, $s_{1} \leq_{F, N} s_{0}$ tal que $s_{1} \mid \sigma_{1} \leq t_{1}$. Como $D$ é aberto denso e $t_{1} \in D$, então $s_{1} \in D$ e seguimos por indução.

É claro que $s^{*}=s_{K-1}$ satisfaz o lema.

Proposição 4.69. Considere $M$ um modelo transitivo e enumerável $M$ para $Z F C$ e $\kappa$ um cardinal. Se $G$ é um $\mathbb{S}^{\kappa}$-genérico sobre $M$, então

$$
M[G] \vDash \check{\omega}_{1}=\left(\omega_{1}\right)^{M} .
$$


Se $M \vDash C H$, então $\mathbb{S}^{\kappa}$ preserva cardinais.

Demonstração. Indicamos [Ba], Teorema 1.11, página 217.

Proposição 4.70. Considere $M$ um modelo transitivo e enumerável $M$ para $Z F C+C H e$ $\kappa$ um cardinal regular. Se $G$ é um $\mathbb{S}^{\kappa}$-genérico sobre $M$, então

$$
M[G] \vDash 2^{\omega}=\check{\kappa}=(\kappa)^{M} .
$$

Demonstração. Indicamos [Ba], Teorema 1.14, página 218. 


\section{Capítulo 5}

\section{$\mathbf{O}$ espaço $l_{\infty} \equiv C(\beta \mathbb{N})$ e a álgebra $\wp(\mathbb{N})$}

Neste capítulo, o objetivo é estudar com detalhes o espaço $l_{\infty}$. O espaço $l_{\infty}$ é um dos mais clássicos e importantes espaços de Banach de dimensão infinita. Isto se deve ao fato de ser simples defini-lo e nem tão simples trabalhar com ele (por exemplo, ele não é separável e, portanto, não tem base de Schauder). Sua importância também se dá por ser o dual do clássico espaço de Banach $l_{1}$ e, portanto, o bidual do espaço $c_{0}$.

Na primeira seção deste capítulo, veremos a demonstração do seguinte teorema, que garante que o $l_{\infty}$ é da forma $C(K)$, onde $K$ é um espaço booleano:

Teorema. O espaço $l_{\infty}$ é isométrico ao espaço $C(\beta \mathbb{N})$.

Com este resultado, temos uma relação entre a álgebra $\wp(\mathbb{N})$, seu espaço de Stone $\beta \mathbb{N}$ e o espaço das funções contínuas sobre $\beta \mathbb{N}$ e podemos observar como as propriedades de cada um destes objetos podem ser traduzidas para os demais. Podemos, enfim, usar resultados sobre espaços de Banach da forma $C(K)$ ao estudar o $l_{\infty}$.

Veremos ainda que o $l_{\infty}$ possui a propriedade de Grothendieck. Assim, temos nosso primeiro exemplo de um espaço da forma $C(K)$ com a propriedade de Grothendieck, o que justifica nosso interesse por tal espaço.

$\mathrm{Na}$ seção seguinte, iniciamos o estudo de algumas propriedades do espaço $\beta \mathbb{N}$ e da álgebra $\wp(\mathbb{N})$, na tentativa de identificar alguma relação entre elas e a propriedade de Grothendieck. Existem propriedades de álgebras de Boole $A$ que podem ser traduzidas a propriedades do espaço $C(S(A))$. Tentamos aqui verificar se algumas propriedades da 
álgebra $\wp(\mathbb{N})$ podem ser traduzidas a propriedades de $l_{\infty}$, mesmo que esta tradução não ocorra em geral para álgebras de Boole quaisquer com tal propriedade.

Por fim, na última seção, analisamos os possíveis subespaços complementados do $l_{\infty}$, com o seguinte resultado que determina tais espaços:

Teorema. $l_{\infty}$ é primo, isto é, todo subespaço complementado em $l_{\infty}$ de dimensão infinita é isomorfo a $l_{\infty}$.

O estudo dos possíveis subespaços complementados do $l_{\infty}$ tem sua motivação em dois fatos: o primeiro é que, como vimos, se $C(K)$ é Grothendieck, então $C(K)$ não tem cópias complementadas de $c_{0}$. Assim, os subespaços complementados em $l_{\infty}$ de dimensão infinita devem ser, de certa forma, mais complicados que o $c_{0}$. Pretendemos, portanto, entender quão complicados eles são.

A segunda motivação para o estudo dos possíveis subespaços complementados do $l_{\infty}$ é a história da busca por espaços de Banach de dimensão infinita, que possuem apenas subespaços complementados triviais, isto é, de dimensão ou codimensão finita. Pełczyński mostrou que os espaços $c_{0}$ e $l_{p}$, para $1 \leq p<\infty$, são primos e conjecturou que $l_{\infty}$ fosse primo. Posteriormente, Lindenstrauss provou esta conjectura, e em 1970 conjecturou que todo espaço de Banach possuísse subespaços complementados não triviais, ou seja, de dimensão finita ou cofinita. Somente em 1994, Gowers provou que esta conjectura é falsa, e ganhou a Medalha Fields por seu trabalho. Na realidade, ele mostrou algo mais forte: ele construiu um espaço de Banach hereditariamente indecomponível, isto é, tal que todo seu subespaço não é soma direta de dois subespaços de dimensão infinita, ou seja, todo seu subespaço não tem subespaços complementados não triviais. Não pretendemos, porém, mostrar tal resultado. Mostraremos apenas que o $l_{\infty}$ é primo, encerrando este capítulo.

Como referência para os resultados aqui apresentados, indicamos [vM] para as Seções 5.1 e 5.2 e [Ma] para a Seção 5.3.

\subsection{A isometria $l_{\infty} \equiv C(\beta \mathbb{N})$}

O objetivo desta seção é mostrar que o espaço $l_{\infty}$ é um espaço de funções contínuas sobre um espaço booleano. Para isso, precisamos de algumas definições e resultados. 
Definição 5.1. Seja $X$ um espaço topológico Hausdorff e com a propriedade 1 que dados um fechado $F$ em $X$ e um ponto $x \notin F$, existe uma função contínua de $X$ em [0,1] com $\left.f\right|_{F}=0$ e $f(x)=1$. Chamamos de compactificado de Čech-Stone de $X$ (e denotamos por $\beta X)$ um espaço ${ }^{2}$ compacto Hausdorff tal que $X$ é um subespaço topológico denso em $\beta X$ e toda função contínua de $X$ em $[0,1]$ pode ser continuamente estendida a $\beta X$.

Sabe-se que o compactificado de Čech-Stone de um espaço $X$ é único a menos de homeomorfismo. Vejamos então um resultado importante na demonstração do principal teorema desta seção:

Teorema 5.2. O espaço de Stone da álgebra $\wp(\mathbb{N})$ é o compactificado de Čech-Stone de $\mathbb{N}$.

Demonstração. Sabemos que o espaço de Stone de toda álgebra de Boole é Hausdorff e compacto. Para cada $n \in \mathbb{N}$, considere $n^{*}=\{a \subseteq \mathbb{N}: n \in a\}$. Vejamos que cada $n^{*}$ é um ultrafiltro: temos que para todo $a \subseteq \mathbb{N}$, ou $n \in a$ ou $n \in \mathbb{N} \backslash a$. Logo, ou $a \in n^{*}$ ou $\mathbb{N} \backslash a \in n^{*}$.

Para mostrar que o conjunto $\left\{n^{*}: n \in \mathbb{N}\right\}$ é discreto, basta notar que $\left\{n^{*}\right\}=\{n\}^{*}$. Vejamos então que o conjunto $\left\{n^{*}: n \in \mathbb{N}\right\}$ é denso em $S(\wp(\mathbb{N}))$ : para isso, mostremos que tal conjunto intercepta todo aberto-fechado não vazio de $S(\wp(\mathbb{N}))$. Seja então $a \subseteq \mathbb{N}$, $a \neq \emptyset$. Daí, existe $m \in \mathbb{N}$ tal que $m \in a$. Logo, $a \in m^{*}$ e, portanto, $m^{*} \in a^{*}$. Assim, $\left\{n^{*}: n \in \mathbb{N}\right\} \cap a^{*} \supseteq\left\{m^{*}\right\} \neq \emptyset$.

Por fim, considere $f:\left\{n^{*}: n \in \mathbb{N}\right\} \rightarrow[0,1]$ contínua. Para cada $u \in \beta \mathbb{N}$, a família $\{f(a): a \in u\}$ tem a p.i.f.. Como cada $\overline{f(a)}$ é compacto (fechado em um compacto), temos que $\bigcap_{a \in u} \overline{f(a)} \neq \emptyset$. Defina $f(u)$ sendo qualquer elemento de $\bigcap_{a \in u} \overline{f(a)}$ (podemos tomar qualquer valor, pois na verdade este conjunto é unitário). Vejamos que definindo $f$ desta forma, temos que $f$ é contínua: dado $u_{0} \in \beta \mathbb{N}$ e $\varepsilon>0$, seja $a_{0}=\left\{n \in \mathbb{N}:\left|f(n)-f\left(u_{0}\right)\right|<\right.$ $\varepsilon\}$. Mas para todo $a \in u_{0}$ temos que $f(a) \cap\left(f\left(u_{0}\right)-\varepsilon, f\left(u_{0}\right)+\varepsilon\right) \neq \emptyset$. Logo, $a_{0} \cap a \neq \emptyset$ para todo $a \in u_{0}$ e assim, $a_{0} \in u_{0}$. Portanto, $u_{0} \in a_{0}^{*}$ e assim $\left|f(u)-f\left(u_{0}\right)\right| \leq \varepsilon$ para todo $u \in a_{0}^{*}$.

Temos agora os elementos necessários para a demonstração do principal resultado desta

\footnotetext{
${ }^{1}$ Esta propriedade é chamada de $\mathrm{T}_{3 \frac{1}{2}}$, em topologia.

${ }^{2}$ Para todo espaço $T_{3 \frac{1}{2}}$, existe um compactificado de Čech-Stone
} 
seção, que nos fornece fortes ferramentas para trabalhar com o espaço $l_{\infty}$ e justifica nosso interesse por ele:

Teorema 5.3. O espaço $l_{\infty}$ é isométrico ao espaço $C(\beta \mathbb{N})$.

Demonstração. Considere $T: C(\beta \mathbb{N}) \rightarrow l_{\infty}$ definida por

$$
T(f)=\left.f\right|_{\mathbb{N}}
$$

para todo $f \in C(\beta \mathbb{N})$. É claro que $T$ está bem definida, pois $\beta \mathbb{N}$ é compacto e, portanto, se $f \in C(\beta \mathbb{N})$, então $f$ é limitada. É fácil ver que $T$ é linear. $T$ é limitada, pois dada $f \in C(\beta \mathbb{N})$, temos

$$
\|T(f)\|=\sup _{n \in \mathbb{N}} f(n)=\sup _{x \in \beta \mathbb{N}} f(x)=\|f\|,
$$

pois $\mathbb{N}$ é denso em $\beta \mathbb{N}$. Assim, $T$ é limitada, injetora e uma isometria sobre sua imagem.

$T$ é sobrejetora, pois dado $(f(n))_{n \in \mathbb{N}} \in l_{\infty}$, temos que $f$ pode ser estendida a $\beta \mathbb{N}$ continuamente, pela definição de compactificado de Čech-Stone. Portanto, $T$ é uma isometria entre $C(\beta \mathbb{N})$ e $l_{\infty}$.

Teorema 5.4. $l_{\infty}$ tem a propriedade de Grothendieck e do Exemplo 1.25.

Demonstração. Segue diretamente do Corolário 3.48.

\section{$5.2 \quad$ Propriedades de $\wp(\mathbb{N})$ e $\beta \mathbb{N}$}

Pretendemos nesta seção fazer um estudo das propriedades da álgebra $\wp(\mathbb{N})$ e do espaço $\beta \mathbb{N}$ e procurar possíveis traduções de tais propriedades a propriedades do espaço $l_{\infty}$. Sabemos que há uma dualidade entre as propriedades de $\wp(\mathbb{N})$ e de $\beta \mathbb{N}$ e que muitas delas podem ser traduzidas para o $l_{\infty}$.

Definição 5.5. Dizemos que uma álgebra de Boole tem c.c.c. se não tem anticadeias não enumeráveis.

Note que a definição acima coincide com a definição de c.c.c. para ordens parciais que vimos no Capítulo 4. 
Proposição 5.6. $\wp(\mathbb{N})$ tem c.c.c.

Demonstração. Suponhamos, por absurdo, que existe $\left(a_{\alpha}\right)_{\alpha<\omega_{1}} \subseteq \wp(\mathbb{N})$ uma anticadeia. Para cada $\alpha<\omega_{1}$, seja $n_{\alpha} \in a_{\alpha}$ um natural. Temos que se $\alpha<\beta<\omega_{1}$, então $n_{\alpha} \neq n_{\beta}$, pois $a_{\alpha} \cap a_{\beta}=\emptyset$. Daí, teríamos $\omega_{1}$ naturais distintos, um absurdo.

Proposição 5.7. $|\wp(\mathbb{N})|=2^{\omega}$ e, portanto, $\beta \mathbb{N}$ não é metrizável.

Demonstração. Segue diretamente da definição do cardinal $2^{\omega}$ e da Proposição 1.57.

Proposição 5.8. $\wp(\mathbb{N})$ é completa e, portanto, $\beta \mathbb{N}$ é extremamente desconexo.

Demonstração. Segue do Exemplo 1.25 e da Proposição 1.63.

Uma tradução natural para a completude de $\wp(\mathbb{N})$ é a injetividade de $l_{\infty}$. Isto é natural pois a completude de uma álgebra de Boole é equivalente a uma propriedade análoga à injetividade de espaços de Banach: dizemos que uma álgebra de Boole $A$ é injetiva se para quaisquer álgebras $B, C$ tais que $B$ é subálgebra de $C$ e qualquer homomorfismo $h: B \rightarrow A$, existe $\tilde{h}: C \rightarrow A$ um homomorfismo que estende $h$. De fato, esta tradução ocorre, mas não apenas para a álgebra $\wp(\mathbb{N})$ e sim, para toda álgebra completa, como vimos no Teorema de Nachbin-Goodman (3.32). Temos, assim, o primeiro exemplo de tradução.

Proposição 5.9. $\wp(\mathbb{N})$ tem família independente de cardinalidade $2^{\omega}$ e, portanto, $|\beta \mathbb{N}|=$ $2^{2^{\omega}}$.

Demonstração. É claro que $|\beta \mathbb{N}|=|U l t(\wp(\mathbb{N}))| \leq|\wp(\wp(\mathbb{N}))|=2^{2^{\omega}}$. Para a outra desigualdade, pela Proposição 1.60, basta achar uma família independente de cardinalidade $2^{\omega}$ em $\wp(X)$, onde $X$ é um conjunto enumerável.

Temos que $2^{\omega}$ é um espaço topológico ${ }^{3}$ metrizável e, portanto, $C l o p\left(2^{\omega}\right)$ é enumerável. Achemos então uma família independente de cardinalidade $2^{\omega}$ em $\wp\left(C l o p\left(2^{\omega}\right)\right)$. Temos que $\operatorname{Ult}\left(\operatorname{Clop}\left(2^{\omega}\right)\right) \subseteq \wp\left(C \operatorname{lop}\left(2^{\omega}\right)\right)$. Como $2^{\omega}$ é homeomorfo a $S\left(C \operatorname{lop}\left(2^{\omega}\right)\right)$, temos que $\left|S\left(C l o p\left(2^{\omega}\right)\right)\right|=\left|2^{\omega}\right|=2^{\omega}$. Vejamos que $U l t\left(C l o p\left(2^{\omega}\right)\right)$ é uma família independente.

\footnotetext{
${ }^{3}$ Denotamos por $2^{\omega}$ aqui o produto de Tychonoff do espaço discreto $\{0,1\}, \omega$ vezes.
} 
Sejam $u_{1}, \ldots, u_{n} \in \operatorname{Ult}\left(C \operatorname{Clop}\left(2^{\omega}\right)\right)$ e $e:\{1, \ldots, n\} \rightarrow\{-1,1\}$. Usando o fato que $S\left(C l o p\left(2^{\omega}\right)\right)$ é booleano, é possível achar $a \in C l o p\left(2^{\omega}\right)$ tal que $a \in u_{i}$ se, e somente se, $e(i)=1$. Portanto, $\operatorname{Ult}\left(C \operatorname{Cop}\left(2^{\omega}\right)\right)$ é uma família independente em $\wp\left(C l o p\left(2^{\omega}\right)\right)$.

Lema 5.10. Se A é imagem homomorfa infinita de $\wp(\mathbb{N})$, então $|A| \geq 2^{\omega}$.

Demonstração. Seja $h: \wp(\mathbb{N}) \rightarrow A$ um epimorfismo e tomemos $\left(a_{n}\right)_{n \in \mathbb{N}} \subseteq A$ uma anticadeia. Existe então $\left(b_{n}\right)_{n \in \mathbb{N}} \subseteq \wp(\mathbb{N})$ tal que $h\left(b_{n}\right)=a_{n}$. Consideremos $c_{0}=b_{0}$ e, para cada $n \in \mathbb{N}, c_{n+1}=b_{n+1} \backslash \bigcup_{i=0}^{n} b_{i}$. Daí, temos que $c_{n+1}=b_{n+1} \backslash \bigcup_{i=0}^{n}\left(b_{n+1} \cap b_{i}\right)$. Logo,

$$
h\left(c_{n+1}\right)=h\left(b_{n+1} \backslash \bigcup_{i=0}^{n}\left(b_{n+1} \cap b_{i}\right)\right)=h\left(b_{n+1}\right)-\sum_{i=0}^{n} h\left(b_{n+1}\right) \cdot h\left(b_{i}\right)=a_{n+1} .
$$

Assim, temos que $\left(c_{n}\right)_{n \in \mathbb{N}} \subseteq \wp(\mathbb{N})$ é uma anticadeia e $h\left(c_{n}\right)=a_{n}$ para todo $n \in \mathbb{N}$.

Para cada $X \in \wp(\mathbb{N})$, defina $c_{X}=\bigcup_{n \in X} c_{n} \in \wp(\mathbb{N})$. Temos que se $X, Y \in \wp(\mathbb{N})$, $X \neq Y$, então $h\left(c_{X}\right) \neq h\left(c_{Y}\right)$. Daí, $\left\{h\left(c_{X}\right): X \in \wp(\mathbb{N})\right\} \subseteq A$ e, portanto, $|A| \geq 2^{\omega}$.

Proposição 5.11. $\beta \mathbb{N}$ não tem seqüências convergentes não triviais.

Demonstração. Se $\beta \mathbb{N}$ tivesse uma seqüência convergente não trivial, pela dualidade de Stone (1.54) e pela Proposição 1.58, teríamos que $\operatorname{Fin} \operatorname{Cofin}(\mathbb{N})$ seria imagem homomorfa de $\wp(\mathbb{N})$, contradizendo o lema anterior.

Lembramos que vimos (Teorema 3.30) que se $K$ tem seqüências convergentes não triviais, então $C(K)$ tem uma cópia complementada de $c_{0}$. Vimos também (Teorema 3.44) que espaços $C(K)$ com a propriedade de Grothendieck não têm cópias complementadas de $c_{0}$. Como $l_{\infty}=C(\beta \mathbb{N})$ é Grothendieck, $\beta \mathbb{N}$ não poderia, de fato, ter sequiências convergentes não triviais. Mas temos acima uma demonstração direta deste fato.

Notemos que uma tradução natural para o fato que uma álgebra de Boole $A$ não tem imagens homomorfas enumeráveis, seria que $C(S(A))$ é Grothendieck, como acontece neste caso. Porém, esta tradução não acontece sempre, como mostra o Exemplo 4.10 de [Scha].

Proposição 5.12. Se $X \subseteq \beta \mathbb{N}$ é homeomorfo a $\beta \mathbb{N}$, então $X$ é retrato de $\beta \mathbb{N}$. 
Demonstração. Seja $h: \wp(\mathbb{N}) \rightarrow \wp(\mathbb{N})$ um epimorfismo. Considere $\left(a_{n}\right)_{n \in \mathbb{N}} \subseteq \wp(\mathbb{N})$ uma anticadeia tal que $\bigcup_{n \in \mathbb{N}} a_{n}=\mathbb{N}$ e $h\left(a_{n}\right)=\{n\}$. Seja então $A=\left\{\bigcup_{n \in X} a_{n}: X \in \wp(\mathbb{N})\right\}$. Vejamos que $A$ é uma subálgebra de $\wp(\mathbb{N})$. Para isso, considere $X, Y \subseteq \mathbb{N}$ e temos:

- $\left(\bigcup_{n \in X} a_{n}\right) \cup\left(\bigcup_{n \in Y} a_{n}\right)=\bigcup_{n \in X \cup Y} a_{n}$;

- $\left(\bigcup_{n \in X} a_{n}\right) \cap\left(\bigcup_{n \in Y} a_{n}\right)=\bigcup_{n \in X \cap Y} a_{n}$, uma vez que $a_{n}$ 's são dois a dois disjuntos;

- $\mathbb{N} \backslash\left(\bigcup_{n \in X} a_{n}\right)=\bigcup_{n \in \mathbb{N} \backslash X} a_{n}$, uma vez que $a_{n}$ 's são dois a dois disjuntos e $\bigcup_{n \in \mathbb{N}} a_{n}=\mathbb{N}$;

- $\mathbb{N}=\bigcup_{n \in \mathbb{N}} a_{n}$ e $\emptyset=\bigcup_{n \in \emptyset} a_{n}$.

Portanto, $A$ é subálgebra de $\wp(\mathbb{N})$. Vejamos que $\left.h\right|_{A}$ é isomorfismo de $A$ sobre $\wp(\mathbb{N})$. Já sabemos que é homomorfismo. Vejamos que se $X \in \wp(\mathbb{N})$ então $h\left(\bigcup_{n \in X} a_{n}\right)=X$. Temos que se $k \in X$, então $\{k\}=h\left(a_{k}\right) \subseteq h\left(\bigcup_{n \in X} a_{n}\right)$ e se $k \notin X$, então $\{k\}=h\left(a_{k}\right) \subseteq$ $h\left(\bigcup_{n \in \mathbb{N} \backslash X}\right)$. Daí, é fácil ver que $\left.h\right|_{A}$ é um isomorfismo de $A$ sobre $\wp(\mathbb{N})$.

Assim, temos que todo epimorfismo $h: \wp(\mathbb{N}) \rightarrow \wp(\mathbb{N})$ é um retrato. Em particular, pela Proposição 1.69 , temos que todo subespaço de $\beta \mathbb{N}$ homeomorfo a $\beta \mathbb{N}$ é um retrato.

Proposição 5.13. Se $X \subseteq \beta \mathbb{N}$ é homeomorfo a $\omega^{*}$, então $X$ não é retrato de $\beta \mathbb{N}$.

Demonstração. Suponhamos, por absurdo, que existe $X \subseteq \beta \mathbb{N}$ homeomorfo a $\omega^{*}$ e $X$ é retrato de $\beta \mathbb{N}$. Daí, pela Proposição 1.69, temos que existe $h: \wp(\mathbb{N}) \rightarrow \wp(\mathbb{N}) /$ Fin um retrato, isto é, existe $A \subseteq \wp(\mathbb{N})$ subálgebra tal que $\left.h\right|_{A}$ é isomorfismo. Logo, temos $\left(a_{\alpha}\right)_{\alpha<2^{\omega}} \subseteq A$ uma anticadeia, contradizendo o fato que $\wp(\mathbb{N})$ tem c.c.c. (Proposição $5.6)$.

Proposição 5.14. Se $X \subseteq \beta \mathbb{N}$ é discreto e enumerável, então $\bar{X}$ é homeomorfo a $\beta \mathbb{N}$.

Demonstração. Seja $X=\left\{u_{n}: n \in \mathbb{N}\right\} \subseteq \beta \mathbb{N}$ um subespaço discreto. Daí, existem $b_{n} \in \wp(\mathbb{N})$ tais que para todos $n, m \in \mathbb{N}, m \neq n, b_{n}^{*} \cap b_{m}^{*} \cap X=\emptyset$ e $u_{n} \in b_{n}^{*}$. Tome $a_{n}=b_{n} \backslash \bigcup_{i<n} b_{i} \in \wp(\mathbb{N})$ e temos que para todos $n, m \in \mathbb{N}, m \neq n, a_{n}^{*} \cap a_{m}^{*}=\emptyset \mathrm{e} u_{n} \in a_{n}^{*}$.

Defina $h: \wp(\mathbb{N}) \rightarrow C l o p(\bar{X})$ por $h(b)=\left(\bigcup_{n \in b} a_{n}\right)^{*} \cap \bar{X}$. É claro que $h$ está bem definida. Vejamos que $h$ é um isomorfismo. $h$ é um homomorfismo, pois dados $b_{1}, b_{1} \in$ $\wp(\mathbb{N})$, temos 
- $\left.h\left(b_{1} \cup b_{2}\right)=\left(\bigcup_{n \in b_{1} \cup b_{2}} a_{n}\right)^{*} \cap \bar{X}=\left(\left(\bigcup_{n \in b_{1}} a_{n}\right) \cup \bigcup_{n \in b_{2}} a_{n}\right)\right)^{*} \cap \bar{X}$

$$
\left.=\left(\left(\bigcup_{n \in b_{1}} a_{n}\right)^{*} \cup \bigcup_{n \in b_{2}} a_{n}\right)^{*}\right) \cap \bar{X}=h\left(b_{1}\right) \cup h\left(b_{2}\right) ;
$$

- o produto é análogo;

- $h\left(\mathbb{N} \backslash b_{1}\right)=\left(\bigcup_{n \in \mathbb{N} \backslash b_{1}} a_{n}\right)^{*} \cap \bar{X}=\left(\beta \mathbb{N} \backslash\left(\bigcup_{n \in b_{1}} a_{n}\right)^{*}\right) \cap \bar{X}$

$$
=\bar{X} \backslash\left(\left(\bigcup_{n \in b_{1}} a_{n}\right)^{*}\right) \cap \bar{X}=\bar{X} \backslash h\left(b_{1}\right)=-h\left(b_{1}\right) ;
$$

- $h(\emptyset)=\left(\bigcup_{n \in \emptyset} a_{n}\right)^{*} \cap \bar{X}=\emptyset$ e $h(\mathbb{N})=\left(\bigcup_{n \in \mathbb{N}} a_{n}\right)^{*} \cap \bar{X} \supseteq\left\{u_{n}: n \in \mathbb{N}\right\}$ e é fechado. Logo, $h(\mathbb{N})=\bar{X}$.

Assim, temos que $h$ é um homomorfismo. $h$ é injetor, pois se $b \in \wp(\mathbb{N}) \backslash \emptyset$, tomemos $k \in b$. Daí, $u_{k} \in a_{k}^{*} \cap X \subseteq\left(\bigcup_{n \in b} a_{n}\right)^{*} \cap \bar{X}$ e daí, $h(b) \neq \emptyset$.

Por fim, vejamos que $h$ é sobrejetor. Tomemos $A \in \operatorname{Clop}(\bar{X})$. Então, $A=a^{*} \cap \bar{X}$, para algum $a \in \wp(\mathbb{N})$. Tomemos $b=\left\{n \in \mathbb{N}: a \in u_{n}\right\} \in \wp(\mathbb{N})$. Vejamos que $h(b)=A$. Por um lado, se $u_{k} \in A=a^{*} \cap \bar{X}$, então $a \in u_{k}$ e daí, $k \in b$. Logo, $u_{k} \in a_{k}^{*} \cap X \subseteq$ $\left(\bigcup_{n \in b} a_{n}\right)^{*} \cap \bar{X}=h(b)$. Reciprocamente, se $u_{k} \in h(b)$, temos que $a_{k}, \bigcup_{n \in b} a_{n} \in u_{k} \mathrm{e}$, portanto, $a_{k} \cap \bigcup_{n \in b} a_{n} \neq \emptyset$. Como $a_{n}$ 's são dois a dois disjuntos, temos que $k \in b$ e então, $u_{k} \in a^{*} \cap \bar{X}=A$.

Assim, $A \cap X=h(b) \cap X$. Como ambos são abertos-fechados em $\bar{X}$ e $X$ é denso, segue que $A=h(b)$ e, portanto, $h$ é sobrejetor, como queríamos.

\subsection{Subespaços complementados do $l_{\infty}$}

O objetivo desta terceira parte do estudo do espaço $l_{\infty}$ é dedicado a seus subespaços complementados. Estudar os subespaços complementados de um espaço de Banach é, em Análise, um importante método para obter propriedades do espaço todo. Assim, veremos que o $l_{\infty}$ é um espaço de certa forma especial neste sentido, por ter a propriedade que todo subespaço complementado de dimensão infinita é isomorfo ao espaço todo.

Sabendo que $l_{\infty}$ tem a propriedade de Grothendieck e que um espaço $C(K)$ tem tal propriedade se, e somente se, não possui cópias complementadas de $c_{0}$, temos a Proposição 3.26 trivialmente. Porém, achamos preferível apresentar uma demonstração direta desta 
proposição que, como dissemos, foi importante na descoberta do Teorema de Lindenstrauss, nosso próximo objetivo. Para mostrá-lo, precisamos do seguinte:

Lema 5.15. Se $X=l_{\infty}$, ent $\tilde{a} o^{4} l_{\infty}(X) \equiv l_{\infty}$.

Demonstração. Seja $X=l_{\infty}$. Tomemos $\left(a_{n}\right)_{n \in \mathbb{N}} \subseteq \wp(\mathbb{N})$ uma anticadeia de conjuntos infinitos de $\mathbb{N}$ tais que $\bigcup_{n \in \mathbb{N}} a_{n}=\mathbb{N}$. Como cada $a_{n}$ é infinito, temos que $a_{n}^{*}$ é homeomorfo a $\beta \mathbb{N}$ e, portanto, $C\left(a_{n}^{*}\right)$ é isométrico a $C(\beta \mathbb{N})=l_{\infty}$. Consideremos $T_{n}: C\left(a_{n}^{*}\right) \rightarrow l_{\infty}$ uma isometria.

Definamos $T: C(\beta \mathbb{N}) \rightarrow l_{\infty}(X)$ por $T(f)=\left(T_{n}\left(\left.f\right|_{a_{n}^{*}}\right)\right)_{n \in \mathbb{N}}$. É claro que $T$ está bem definida e que é linear. $T$ é injetora, pois se $T(f)=0$, então $T_{n}\left(\left.f\right|_{a_{n}^{*}}\right)=0$ para todo $n \in \mathbb{N}$. Como $T_{n}$ é isometria, segue que $\left.f\right|_{a_{n}^{*}}=0$ para todo $n \in \mathbb{N}$. Logo, $f_{\cup_{n \in \mathbb{N}} a_{n}^{*}}=0$. Como $\mathbb{N} \subseteq \bigcup_{n \in \mathbb{N}} a_{n}^{*}$, temos que $\bigcup_{n \in \mathbb{N}} a_{n}^{*}$ é denso em $\beta \mathbb{N}$ e, portanto, $f=0$.

Para mostrar que $T$ é sobrejetora, considere $\left(f_{n}\right)_{n \in \mathbb{N}} \in l_{\infty}(X)$. Tomemos $f \in l_{\infty}$ dada por $f(k)=T_{n}^{-1}\left(f_{n}\right)(k)$, se $k \in a_{n}^{*}$. É fácil ver que $f$ está bem definida. Daí, tomemos $\tilde{f} \in C(\beta \mathbb{N})$ tal que $\left.\tilde{f}\right|_{\mathbb{N}}=f$. Assim, $T(\tilde{f})=\left(T_{n}\left(\left.f\right|_{a_{n}^{*}}\right)\right)_{n \in \mathbb{N}}=\left(f_{n}\right)_{n \in \mathbb{N}}$.

Por fim, notemos que se $f \in C(\beta \mathbb{N})$, temos

$$
\begin{aligned}
\|T(f)\| & =\left\|\left(T_{n}\left(\left.f\right|_{a_{n}^{*}}\right)\right)_{n \in \mathbb{N}}\right\|=\sup _{n \in \mathbb{N}}\left\|T_{n}\left(\left.f\right|_{a_{n}^{*}}\right)\right\|=\sup _{n \in \mathbb{N}}\left\|\left.f\right|_{a_{n}^{*}}\right\| \\
& =\sup _{n \in \mathbb{N}}\left(\sup _{x \in a_{n}^{*}}|f(a)|\right)=\sup _{x \in \cup_{n \in \mathbb{N}} a_{n}^{*}}|f(x)|=\sup _{x \in \beta \mathbb{N}}|f(x)|=\|f\| .
\end{aligned}
$$

Portanto, $T$ é uma isometria.

Lema 5.16. Seja $X$ um subespaço complementado de $l_{\infty}$ e suponha que existe $Y$ subespaço de $X, \operatorname{com} Y \sim l_{\infty}$. Então, $X \sim l_{\infty}$.

Demonstração. Pelo Teorema 3.34, temos que $Y$ é complementado em $X$. Como $l_{\infty} \sim$ $l_{\infty}(X)$, onde $X=l_{\infty}$, pela Proposição 2.34, segue que $X \sim l_{\infty}$.

Proposição 5.17. Seja $T: l_{\infty} \rightarrow X$ uma aplicação linear limitada, onde $X$ é um espaço de Banach. Suponha que $T: c_{0} \rightarrow T\left[c_{0}\right]$ é um isomorfismo. Então, existe $M \subseteq \mathbb{N}$ infinito tal que $T: l_{\infty}[M] \rightarrow T\left[l_{\infty}[M]\right]$ é um isomorfismo, onde

$$
l_{\infty}[M]=\left\{\left(\alpha_{n}\right)_{n \in N}: \sup _{n \in M}\left|\alpha_{n}\right|<\infty e \forall n \in \mathbb{N} \backslash M \alpha_{n}=0\right\},
$$

com a norma do supremo.

\footnotetext{
${ }^{4}$ Lembramos que $l_{\infty}(X)$ é o espaço de Banach das seqüências limitadas em $X$, com a norma do supremo.
} 
Demonstração. Seja $\left(e_{n}\right)_{n \in \mathbb{N}}$ a seqüência de vetores unitários de $c_{0}$. Para cada $n \in \mathbb{N}$ existe, pelo Teorema de Hahn-Banach (2.5), $f_{n} \in X^{*}$ tal que $f_{n}\left(T\left(e_{n}\right)\right)=1$ e $\left\|f_{n}\right\|=$ $\left\|T\left(e_{n}\right)\right\|^{-1}$. Tomemos então $\mu_{n}=T^{*}\left(f_{n}\right)$. Como $T$ é isomorfismo sobre $c_{0}$, temos que $\sup _{n \in \mathbb{N}}\left\|\mu_{n}\right\|<\infty$.

Como cada $\mu_{n} \in l_{\infty}^{*}$, pelo Teorema de Representação de Riesz (3.13), temos que cada $\mu_{n}$ é uma medida finitamente aditiva sobre $\wp(\mathbb{N})$. Daí, pelo Teorema 3.35 , existe $L \subseteq \mathbb{N}$ infinito tal que para todo $M \subseteq L$ e todo $k \in L$ tem-se que

$$
\mu_{k}(M)=\sum_{n \in M} \mu_{k}(\{n\})
$$

Então, pelo Lema de Rosenthal (3.36), existe $M \subseteq L$ infinito tal que para todo $k \in M$

$$
\sum_{n \in M \backslash\{k\}}\left|\mu_{k}(\{n\})\right|<\frac{1}{2} .
$$

Vejamos que $T: l_{\infty}[M] \rightarrow T\left[l_{\infty}[M]\right]$ é um isomorfismo. Primeiramente, vejamos que é uma injeção: tomemos $\alpha=\left(\alpha_{n}\right)_{n \in \mathbb{N}} \in l_{\infty}[M]$. Temos que $\alpha_{n}=0$, para $n \in \mathbb{N} \backslash M$. Suponha $\alpha \neq 0$. Então

$$
T(\alpha)=\sum_{n \in M} \alpha_{n} T\left(e_{n}\right)
$$

Daí, para cada $k \in M$, temos que

$$
f_{k}(T(\alpha))=f_{k}\left(\sum_{n \in M} \alpha_{n} T\left(e_{n}\right)\right)=\sum_{n \in M} \alpha_{n} f_{k}\left(T\left(e_{n}\right)\right)=\alpha_{k}+\sum_{n \in M \backslash\{k\}} \alpha_{n} f_{k}\left(T\left(e_{n}\right)\right),
$$

pois $f_{k}\left(T\left(e_{k}\right)\right)=1$.

Assim,

$$
\begin{aligned}
\left\|f_{k}\right\| \cdot\|T(\alpha)\| & \geq\left\|f_{k}(T(\alpha))\right\| \geq\left|\alpha_{k}\right|-\sum_{n \in M \backslash\{k\}}\left|\alpha_{n}\right|\left|f_{k}\left(T\left(e_{n}\right)\right)\right| \\
& =\left|\alpha_{k}\right|-\sum_{n \in M \backslash\{k\}}\left|\alpha_{n}\right| \mid\left(T^{*}\left(f_{k}\right)\left(e_{n}\right)|\geq| \alpha_{k}\left|-\|\alpha\| \sum_{n \in M \backslash\{k\}}\right| T^{*}\left(f_{k}\right)\left(e_{n}\right) \mid\right. \\
& =\left|\alpha_{k}\right|-\|\alpha\| \sum_{n \in M \backslash\{k\}}\left|\mu_{k}(\{n\})\right| \geq\left|\alpha_{k}\right|-\|\alpha\| \frac{1}{2} .
\end{aligned}
$$

Como $\left.T\right|_{c_{0}}$ é isomorfismo e $\left\|f_{k}\right\|=\left\|T\left(e_{k}\right)\right\|^{-1}$, temos que

$$
\sup _{k \in M}\left\|f_{k}\right\| \leq \sup _{n \in \mathbb{N}}\left\|f_{k}\right\| \leq \sup _{n \in \mathbb{N}}\left\|T\left(e_{k}\right)\right\|^{-1}=r<\infty .
$$

Portanto,

$$
r \cdot\|T(\alpha)\| \geq \sup _{n \in M}\left|\alpha_{n}\right|-\frac{\|\alpha\|}{2}=\frac{\|\alpha\|}{2} .
$$


Assim, temos que $\|T(\alpha)\|>0$ e, portanto, $T(\alpha) \neq 0$. Logo, $\left.T\right|_{l_{\infty}[M]}$ é injetora. Já sabemos que $T$ é limitada e, portanto, $\left.T\right|_{l_{\infty}[M]}$ é limitada. Para concluirmos que $\left.T\right|_{l_{\infty}[M]}$ é um isomorfismo sobre sua imagem, basta notar que, por $(*), T^{-1}$ é limitada. Portanto, temos o resultado.

Proposição 5.18. Suponha $X$ um subespaço complementado em $l_{\infty}$ tal que existe $Y \subseteq X$ com $Y \sim c_{0}$. Então $X \sim l_{\infty}$.

Demonstração. Seja $P: l_{\infty} \rightarrow X$ uma projeção. Como temos $Y \subseteq X$ com $Y \sim c_{0}$, segue que $P: Y \rightarrow Y$ é um isomorfismo (na verdade, é a identidade). Pela proposição anterior, temos que existe $Z \subseteq X$ tal que $Z \sim l_{\infty}$. Daí, pelo Lema 5.16, segue que $X \sim l_{\infty}$.

Teorema 5.19 (Lindenstrauss). $O$ espaço $l_{\infty}$ é primo, isto é, todo subespaço complementado em $l_{\infty}$ de dimensão infinita é isomorfo a $l_{\infty}$.

Demonstração. Seja $X$ um subespaço complementado de $l_{\infty}$ de dimensão infinita. Pelo Corolário 3.43, existe $Y$ um subespaço de $X$ isomorfo a $c_{0}$. Daí, pela proposição anterior, $X \sim l_{\infty}$.

Convém notar que, apesar deste resultado, $\wp(\mathbb{N})$ tem como retrato uma álgebra $A$ infinita que não é isomorfa a $\wp(\mathbb{N})$, ilustrando que nem todas as propriedades de $\wp(\mathbb{N})$ podem ser traduzidas de forma natural para $l_{\infty}$. Além disso, existe $A$ uma álgebra de Boole não isomorfa a $\wp(\mathbb{N})$ tal que $C(S(A))$ é complementado em $l_{\infty}$ e, portanto, $C(S(A)) \sim l_{\infty}$. 


\section{Capítulo 6}

\section{$\mathrm{O}$ espaço $l_{\infty} / c_{0} \equiv C\left(\omega^{*}\right)$ e a álgebra $\wp(N) / F$ in}

Nosso objetivo neste capítulo é análogo ao do capítulo anterior: fazer um estudo detalhado do espaço $l_{\infty} / c_{0}$. Este espaço é outro clássico espaço de Banach de dimensão infinita. Apesar de ser o quociente do $l_{\infty}$, um espaço relativamente simples, o $l_{\infty} / c_{0}$ tem diversas propriedades peculiares. O primeiro importante resultado é o seguinte:

Teorema. O espaço $l_{\infty} / c_{0}$ é isométrico ao espaço $C\left(\omega^{*}\right)$.

Tal teorema relaciona o espaço $l_{\infty} / c_{0}$ à álgebra $\wp(\mathbb{N}) / F i n$, uma vez que $\omega^{*}$ é o espaço de Stone desta. Pretendemos, novamente, analisar as propriedades que podem ser traduzidas entre a álgebra de Boole $\wp(\mathbb{N}) / F$ in e os espaços $\omega^{*}$ e $l_{\infty} / c_{0}$. Novamente, como no capítulo anterior, provamos que o $l_{\infty} / c_{0}$ é um espaço de Grothendieck, justificando nosso interesse por ele.

Fazemos então, na seção seguinte, um estudo das propriedades de $\omega^{*}$ e de $\wp(\mathbb{N}) / F i n$. Este estudo visa obter informações sobre mais um espaço de Grothendieck da forma $C(K)$, a fim de compará-las àquelas obtidas sobre o $l_{\infty}$, para posteriormente fazer conjecturas acerca de espaços de Grothendieck da forma $C(K)$.

Aqui, diferentemente do que ocorre com o $l_{\infty}$, já aparece uma propriedade que depende de hipóteses conjuntísticas adicionais, ou seja, que vale ou não, dependendo do modelo em que trabalhamos. 
$\mathrm{Na}$ última seção, analisamos os possíveis subespaços complementados de $l_{\infty} / c_{0}$, tendo as mesmas motivações que no caso do $l_{\infty}$. Porém, os resultados obtidos aqui são, de certa forma, mais fracos que aqueles. Na realidade, o $l_{\infty} / c_{0}$ tem propriedades um pouco mais fracas que o $l_{\infty}$ neste sentido.

O primeiro resultado garante a existência de decomposições $l_{\infty} / c_{0} \sim l_{\infty} \oplus l_{\infty} / c_{0}$ e daí segue que o $l_{\infty}$ é complementado no $l_{\infty} / c_{0}$. Temos assim dois espaços de Grothendieck que possuem o $l_{\infty}$ como subespaço complementado. Veremos no Capítulo 7 que existem espaços de Grothendieck da forma $C(K) \operatorname{sem} l_{\infty}$ como subespaço complementado. Porém, o primeiro exemplo de tal espaço surgiu apenas em 1981, em [Ha].

Por fim, temos o seguinte resultado de consistência:

Teorema. (CH) $l_{\infty} / c_{0}$ é primário, isto é, se $A, B$ são espaços de Banach e $l_{\infty} / c_{0} \sim A \oplus B$, então ou $A \sim l_{\infty} / c_{0}$ ou $B \sim l_{\infty} / c_{0}$.

Convém notar que a propriedade de ser primário é mais fraca que a propriedade de ser primo. Para que um espaço seja primo, exigimos que cada seu subespaço complementado de dimensão infinita seja isomorfo ao espaço todo. Para que um espaço seja primário, basta que em toda sua decomposição em dois espaços, um deles seja isomorfo ao espaço todo, mesmo que o outro não o seja.

Este resultado está demonstrado no artigo [DR] e completa este capítulo. Convém destacar que não se sabe se este fato pode ser provado sem $\mathrm{CH}$, nem se conhece decomposições $A \oplus B$ de $l_{\infty} / c_{0}$, onde $A$ e $B$ não são isomorfos a $l_{\infty} / c_{0}$.

Como principais referências para os resultados abordados neste capítulo, indicamos [LW] e [DR].

\subsection{A isometria $l_{\infty} / c_{0} \equiv C\left(\omega^{*}\right)$}

Para entender a estrutura do espaço $l_{\infty} / c_{0}$, pretendemos mostrar que $l_{\infty} / c_{0} \equiv C\left(\omega^{*}\right)$. Para isso, precisamos primeiramente da seguinte definição:

Definição 6.1. Considere o espaço $\beta \mathbb{N}$. Sabemos que $\mathbb{N}$ é um subespaço denso de $\beta \mathbb{N}$. Chamamos de $\omega^{*}$ ao subespaço $\beta \mathbb{N} \backslash \mathbb{N}$ de $\beta \mathbb{N}$.

\footnotetext{
${ }^{1}$ Convém mencionar que esta notação $\omega^{*}$ poderia ser confundida com o aberto-fechado $\mathbb{N}^{*}$ de $\beta \mathbb{N}$.
} 
Vejamos então que o espaço topológico assim definido é o espaço de Stone da álgebra de Boole $\wp(\mathbb{N}) / F$ in:

Teorema 6.2. O espaço de Stone da álgebra $\wp(\mathbb{N}) /$ Fin é o espaço $\omega^{*}$.

Demonstração. Para mostrar o resultado, é suficiente mostrar que $C l o p\left(\omega^{*}\right)$ é isomorfo a $\wp(\mathbb{N}) / F$ in. Considere então $h: \wp(\mathbb{N}) / F$ in $\rightarrow C l o p\left(\omega^{*}\right)$ definido por $h([a])=a^{*} \cap \omega^{*}$. $h$ está bem definida, pois dado um elemento de $\wp(\mathbb{N}) / F$ in, ele é da forma $[a]$, para algum $a \in \wp(\mathbb{N})$. Daí, $a^{*} \in C l o p(\beta \mathbb{N})$ e, portanto, $a^{*} \cap \omega^{*} \in C l o p\left(\omega^{*}\right)$.

Vejamos que $h$ é um homomorfismo: dados $a, b \in \wp(\mathbb{N})$, temos

- $h([a]+[b])=h([a+b])=(a+b)^{*} \cap \omega^{*}=\left\{u \in \omega^{*}: a+b \in u\right\}=\left\{u \in \omega^{*}: a \in\right.$ $u\} \cup\left\{u \in \omega^{*}: b \in u\right\}=\left(a^{*} \cap \omega^{*}\right) \cup\left(b^{*} \cap \omega^{*}\right)=h([a]) \cup h([b]) ;$

- analogamente para o produto;

- $h(-[a])=h([\mathbb{N} \backslash a])=(\mathbb{N} \backslash a)^{*} \cap \omega^{*}=\left(\beta \mathbb{N} \backslash a^{*}\right) \cap \omega^{*}=\omega^{*} \backslash a^{*}=-h([a]) ;$

- $h([\emptyset])=\emptyset^{*} \cap \omega^{*}=\emptyset$ e $h([\mathbb{N}])=\mathbb{N}^{*} \cap \omega^{*}=\beta \mathbb{N} \cap \omega^{*}=\omega^{*}$.

$h$ é injetor, pois se $h([a])=\emptyset$, então $a^{*} \cap \omega^{*}=\emptyset$. Daí, $a$ é finito e portanto, $[a]=[\emptyset]=0$.

Temos, por fim, que $h$ é sobrejetor: seja $U \in C l o p\left(\omega^{*}\right)$. Pela dualidade de Stone, existe $V \in C l o p(\beta \mathbb{N})$ tal que $V \cap \omega^{*}=U$. Daí, existe $a \in \wp(\mathbb{N})$ tal que $V=a^{*}$. Logo, $h([a])=a^{*} \cap \omega^{*}=U \cap \omega^{*}=V$.

Teorema 6.3. O espaço $l_{\infty} / c_{0}$ é isométrico ao espaço $C\left(\omega^{*}\right)$.

Demonstração. Pelo Teorema 3.23, temos que $C\left(\omega^{*}\right)=C(\beta \mathbb{N}) / C_{0}\left(\beta \mathbb{N} \mid \omega^{*}\right)$. Considere então $I: C(\beta \mathbb{N}) \rightarrow l_{\infty}$ a isometria dada pelo Teorema 5.3. Vejamos que $I\left[C_{0}\left(\beta \mathbb{N} \mid \omega^{*}\right)\right]=c_{0}$.

Dada $\left(a_{n}\right)_{n \in \mathbb{N}} \in c_{0}$, temos que a extensão para $\beta \mathbb{N}$ é $0 \mathrm{em} \omega^{*}$. Por outro lado, se $f \in C_{0}\left(\beta \mathbb{N} \mid \omega^{*}\right)$, então á claro que $\lim _{n \rightarrow \infty} f(n)=0$.

Portanto, $C\left(\omega^{*}\right)=C(\beta \mathbb{N}) / C_{0}\left(\beta \mathbb{N} \mid \omega^{*}\right) \equiv l_{\infty} / c_{0}$.

Teorema 6.4. $l_{\infty} / c_{0}$ tem a propriedade de Grothendieck.

Notemos, porém, que neste caso $\mathbb{N}^{*}=\beta \mathbb{N}$. Veremos adiante que $\omega^{*}=S(\wp(\mathbb{N}) / F$ in e isto também poderia dar confusão. Neste caso, temos que $[\mathbb{N}]^{*}=\omega^{*}$. 
Demonstração. Pelo Corolário 5.4, temos que $l_{\infty}$ é Grothendieck. Daí, pelo Teorema 2.43, segue que $l_{\infty} / c_{0}$ é Grothendieck.

\subsection{Propriedades de $\wp(\mathbb{N}) / F$ in e de $\omega^{*}$}

O objetivo desta seção é a apresentação de diversas propriedades da álgebra $\wp(\mathbb{N}) / F$ in e do espaço $\omega^{*}$, a fim de obter informações sobre o espaço $l_{\infty} / c_{0}$. Além de apresentar a demonstração de tais propriedades, buscamos encontrar traduções delas para o espaço $l_{\infty} / c_{0}$.

Proposição 6.5. Existe $\left(a_{\alpha}\right)_{\alpha<2^{\omega}} \subseteq \wp(\mathbb{N}) / F$ in uma anticadeia. Logo, $\wp(\mathbb{N}) / F$ in não tem c.c.c. e, portanto, não é subálgebra de $\wp(\mathbb{N})$.

Demonstração. Considere $\mathbb{Q}=\left\{q_{n}: n \in \mathbb{N}\right\}$ uma enumeração dos números racionais. Para cada $r \in \mathbb{R}$, seja $X_{r} \subseteq \mathbb{Q}$ uma seqüência de racionais que converge para $r$.

Para cada $r \in \mathbb{R}$, defina $a_{r}=\left\{n \in \mathbb{N}: q_{n} \in X_{r}\right\}$. Vejamos que $\left(\left[a_{r}\right]\right)_{r \in \mathbb{R}} \subseteq \wp(\mathbb{N}) / F$ in é uma anticadeia. Dados $r, s \in \mathbb{R}, r<s$, existe $n_{0} \in \mathbb{N}$ tal que se $n \geq n_{0}$, então se $n \in a_{r},\left|r-q_{n}\right|<\frac{s-r}{3}$ e se $n \in a_{s},\left|s-q_{n}\right|<\frac{s-r}{3}$. Logo, $a_{r} \cap a_{s} \subseteq\left\{0, \ldots, n_{0}\right\}$. Portanto, $\left[a_{r}\right] \cap\left[a_{s}\right]=\emptyset$.

Proposição 6.6. $|\wp(\mathbb{N}) / F i n|=2^{\omega}$ e, portanto, $\omega^{*}$ não é metrizável.

Demonstração. Temos, da proposição anterior, que $|\wp(\mathbb{N}) / F i n| \geq 2^{\omega}$. Por outro lado, $\wp(\mathbb{N}) / F$ in é imagem homomorfa de $\wp(\mathbb{N})$ e $|\wp(\mathbb{N})|=2^{\omega}$. Logo, $|\wp(\mathbb{N}) / F i n|=2^{\omega}$. Segue, da Proposição 1.57, que $\omega^{*}$ não é metrizável.

Proposição 6.7. $\wp(\mathbb{N}) /$ Fin não é completa e, portanto, $\omega^{*}$ não é extremamente desconexo.

Demonstração. Seja $\left(a_{n}\right)_{n \in \mathbb{N}} \subseteq \wp(\mathbb{N}) / F i n$ uma anticadeia e seja $\left(b_{n}\right)_{n \in \mathbb{N}} \subseteq \wp(\mathbb{N})$ uma anticadeia tal que $a_{n}=\left[b_{n}\right]$. Seja $a \in \wp(\mathbb{N}) / F$ in tal que $a_{n} \leq a$ para todo $n \in \mathbb{N}$. Tomemos $b \in \wp(\mathbb{N})$ tal que $a=[b]$. Para cada $n \in \mathbb{N}$, seja $k_{n} \in b \cap b_{n}$ e defina $c=b \backslash\left\{k_{n}: n \in \mathbb{N}\right\}$. Temos que $[c]<[b]$, pois $\left\{k_{n}: n \in \mathbb{N}\right\}$ é infinito e $b_{n} \backslash c=\left(b_{n} \backslash b\right) \cup\left\{k_{n}\right\}$ é finito. Logo, para todo $n \in \mathbb{N}, a_{n}=\left[b_{n}\right] \leq[c]$. Portanto, $a \neq \sum_{n \in \mathbb{N}} a_{n}$. Segue, da Proposição 1.63, que $\omega^{*}$ não é extremamente desconexo. 
Definição 6.8. Seja A uma álgebra de Boole. Dizemos que $\left(a_{n}\right)_{n \in \mathbb{N}} \subseteq A$ é uma $(\omega, 1)$ abertura se $a_{n+1}<a_{n}$ para todo $n \in \mathbb{N}$, mas não existe $x>0$ tal que $x<a_{n}$ para todo $n \in \mathbb{N}$. Dizemos que $\left(a_{n}\right)_{n \in \mathbb{N}},\left(b_{n}\right)_{n \in \mathbb{N}} \subseteq$ A formam uma $(\omega, \omega)$-abertura se $a_{n}<a_{n+1}<$ $b_{n+1}<b_{n}$ para todo $n \in \mathbb{N}$, mas não existe $x \in A$ tal que $a_{n}<x<b_{n}$ para todo $n \in \mathbb{N}$.

Proposição 6.9. $\wp(\mathbb{N}) /$ Fin não tem $(\omega, \omega)$-aberturas nem $(\omega, 1)$-aberturas.

Demonstração. Vejamos que $\wp(\mathbb{N}) / F i n$ não tem $(\omega, 1)$-aberturas. Para isso, considere$\operatorname{mos}\left(\left[a_{n}\right]\right)_{n \in \mathbb{N}} \subseteq \wp(\mathbb{N}) / F$ in decrescente. É fácil ver que podemos supor, sem perda de generalidade, que $a_{n+1} \subseteq a_{n}$.

Tome $k_{0} \in a_{0}$ qualquer e tome $k_{n+1} \in a_{n+1} \backslash\left\{k_{i}: 0 \leq i \leq n\right\}$. Seja $x=\left\{k_{n}: n \in \mathbb{N}\right\}$. Temos que $x \backslash a_{n} \subseteq\left\{k_{0}, \ldots, k_{n-1}\right\}$ e, portanto, é finito. Logo, $[x] \leq\left[a_{n+1}\right]<\left[a_{n}\right]$ para todo $n \in \mathbb{N}$.

Mostremos então que $\wp(\mathbb{N}) / F$ in não tem $(\omega, \omega)$-aberturas. Sejam $A=\left\{\left[a_{n}\right]: n \in \mathbb{N}\right\}$ e $B=\left\{\left[b_{n}\right]: n \in \mathbb{N}\right\}$ em $\wp(\mathbb{N}) / F$ in $\left(a_{n}, b_{n} \in \wp(\mathbb{N})\right)$, tais que

$$
\forall n \in \mathbb{N} \quad\left[a_{n}\right]<\left[a_{n+1}\right]<\left[b_{n+1}\right]<\left[b_{n}\right]
$$

Podemos supor, sem perda de generalidade, que para todo $n \in \mathbb{N}, a_{n} \subseteq a_{n+1}$ e $b_{n+1} \subseteq b_{n}$.

Tomemos $x=\bigcup_{n \in \mathbb{N}} a_{n} \cap b_{n}$. Temos que para todo $n \in \mathbb{N}, a_{n} \backslash x \subseteq a_{n} \backslash b_{n}$ que é finito. Logo, para todo $n \in \mathbb{N},\left[a_{n}\right]<\left[a_{n}+1\right] \leq[x]$.

Por outro lado, para todo $n \in \mathbb{N}$,

$$
x \backslash b_{n} \subseteq\left(\bigcup_{i<n} a_{i} \cap b_{i}\right) \backslash b_{n} \subseteq \bigcup_{i<n}\left(a_{i} \backslash b_{n}\right)
$$

que é finito. Portanto, para todo $n \in \mathbb{N},[x] \leq\left[b_{n+1}\right]<\left[b_{n}\right]$.

Corolário 6.10. $\wp(\mathbb{N}) / F$ in tem cadeias bem ordenadas de cardinalidade $\omega_{1}$.

Demonstração. Vamos construir, por indução, $\left(a_{\alpha}\right)_{\alpha<\omega_{1}} \subseteq \wp(\mathbb{N}) / F$ in uma cadeia decrescente.

Seja $\alpha<\omega_{1}$ e suponha que temos $\left(a_{\beta}\right)_{\beta<\alpha} \subseteq \wp(\mathbb{N}) / F i n$ uma cadeia decrescente. Vamos construir $a_{\alpha} \in \wp(\mathbb{N}) / F i n, a_{\alpha}<a_{\beta}$ para todo $\beta<\alpha$. Para isso, consideremos $\left(\alpha_{n}\right)_{n \in \mathbb{N}} \subseteq \alpha$ uma seqüência cofinal em $\alpha$. 
Pela proposição anterior, existe $x \in \wp(\mathbb{N}) / F$ in tal que $x<a_{\alpha_{n}}$ para todo $n \in \mathbb{N}$. Como $\left(\alpha_{n}\right)_{n \in \mathbb{N}}$ é cofinal em $\alpha$, temos que $x<a_{\beta}$ para todo $\beta<\alpha$ (existe, pois $\alpha<\omega_{1}$ ).

A existência de cadeias bem ordenadas em $\wp(\mathbb{N}) / F$ in de cardinalidade $2^{\omega}$ é independente. Assumindo a hipótese do contínuo, temos, pelo corolário anterior que existem tais cadeias. Pode-se mostrar que, no modelo obtido com o forcing de Cohen, não existem tais cadeias (veja [Do2]).

Lema 6.11. Seja A uma álgebra de Boole sem $(\omega, 1)$-aberturas. Para todo a $\in A \backslash\{0\}$, existe uma anticadeia não enumerável abaixo de a.

Demonstração. Seja $\left\{a_{n}: n \in \mathbb{N}\right\}$ uma anticadeia abaixo de $a$. Como $A$ não tem $(\omega, 1)-$ aberturas, existe $x \in A$ tal que $a_{n}<x<a$ para todo $n \in \mathbb{N}$. Daí, $a-x \neq 0$ e, portanto, $\left\{a_{n}: n \in \mathbb{N}\right\} \cup\{a-x\}$ é uma anticadeia que estende a original.

Lema 6.12. Seja $A$ uma álgebra de Boole e suponha que para todo a $\in A \backslash\{0\}$, existe uma anticadeia não enumerável abaixo de a. Se $\left\{a_{n}: n \in \mathbb{N}\right\} \subseteq A \backslash\{0\}$, então existe $\left\{b_{n}: n \in \mathbb{N}\right\}$ uma anticadeia tal que para todo $n \in \mathbb{N}, 0<b_{n} \leq a_{n}$.

Demonstração. Se existe $X$ uma anticadeia tal que para todo $n \in \mathbb{N}$, o conjunto $\left\{x \cdot a_{n}\right.$ : $x \in X\}$ é não enumerável, então tomando $\left\{x_{n}: n \in \mathbb{N}\right\} \subseteq X$ distintos, temos que se $b_{n}=x_{n} \cdot a_{n}$ satisfaz o enunciado.

Vejamos que existe tal anticadeia. Para isso, construímos, por indução, anticadeias $\left(X_{n}\right)_{n \in \mathbb{N}}$ tais que

(i) $X_{n} \subseteq X_{n+1}$;

(ii) para todo $i, n \in \mathbb{N},\left\{x \cdots a_{i}: a \in X_{n}\right\} \backslash\{0\}$ é ou não enumerável, ou vazio;

(iii) para todo $i \leq n,\left\{x \cdot a_{i}: x \in X_{n}\right\}$ é não enumerável.

Daí, tomando $X=\bigcup_{n \in \mathbb{N}} X_{n}$, temos a anticadeia que queremos.

Façamos a construção: dado $X_{n}$, se $\left\{x \cdot a_{n+1}: x \in X_{n}\right\}$ é não enumerável, tomamos $X_{n+1}=X_{n}$. Senão, tomamos $X_{n+1}=X_{n} \cup Y$, onde $Y$ é uma anticadeia não enumerável abaixo de $a_{n+1}$ (que existe pelo lema anterior), subtraída de um conjunto enumerável, de forma a satisfazer (ii). 
Proposição 6.13. Sejam $A, B, C$ álgebras de Boole tais que $A$ é subálgebra de $B,|A| \leq \omega$ e $C$ sem $(\omega, \omega)$-aberturas e sem $(\omega, 1)$-aberturas. Dados $h: A \rightarrow C$ um monomorfismo $e$ $b \in B \backslash A$, existe $\tilde{h}:\langle A \cup\{b\}\rangle \rightarrow C$ um monomorfismo que estende $h$.

Demonstração. Defina $E=\{h(a): a \in A$ e $a \leq b\}$ e $D=\{h(a): a \in A$ e $b \leq a\}$. Pelo Teorema de Extensão de Sikorski (1.28), basta achar $c \in C$ tal que

$$
\forall a, a^{\prime} \in A \quad a \leq b \leq a^{\prime} \Leftrightarrow h(a) \leq c \leq h\left(a^{\prime}\right)
$$

Vejamos que isto é equivalente a achar $c \in C$ tal que

(i) $\forall a, a^{\prime} \in A, a \leq b \leq a^{\prime} \Rightarrow h(a) \leq c \leq h\left(a^{\prime}\right)$;

(ii) $\forall d \in D, \forall x \in h[A], d \cdot x \neq 0 \Rightarrow c \cdot x \neq 0$;

(iii) $\forall e \in E, \forall x \in h[A],(-e) \cdot x \neq 0 \Rightarrow(-c) \cdot x \neq 0$.

Para isso, é suficiente notar que $(i i),($ iii $) \Rightarrow$ a volta de $(i)$. Se $c \leq h(a)$, então $c \cdot(-h(a))=$ 0 e daí, por (ii), existe $d \in D$ tal que $d \cdot(-h(a))=0$. Assim, $d \leq h(a)$ e $d=h\left(a^{\prime}\right)$ para algum $a^{\prime} \geq b$. Portanto, $b \leq a^{\prime} \leq a$, pois $h$ é homomorfismo. A outra desigualdade segue analogamente.

Determinemos então $c \in C$ desta forma. Primeiramente, considere, para cada $x \in$ $h[A]$,

- se $\forall d \in D x \cdot d \neq 0$, então seja $d_{x} \in C$ tal que $\forall d \in D, 0<d_{x}<d \cdot x$;

- se $\forall e \in E x \cdot(-e) \neq 0$, então seja $e_{x} \in C$ tal que $\forall e \in E, 0<e_{x}<(-e) \cdot x$.

Tais $d_{x}$ e $e_{x}$ existem, uma vez que $C$ não tem $(\omega, 1)$-aberturas. Podemos supor, sem perda de generalidade, pelo lema anterior, que $d_{x}$ 's e $e_{x}$ 's são dois a dois disjuntos. Como $|h[A]| \leq|A| \leq \omega$, podemos enumerar $D=\left\{d_{n}: n \in \mathbb{N}\right\}$ e $E=\left\{e_{n}: n \in \mathbb{N}\right\}$. Daí, para todo $n \in \mathbb{N}$, temos

$$
d_{0} \cdots d_{n+1}-\left(e_{0}+\cdots+e_{n+1}\right)<d_{0} \cdots d_{n}-\left(e_{0}+\cdots+e_{n}\right)
$$

Construímos, por indução, $\left(f_{n}\right)_{n \in \mathbb{N}} \in C \backslash\{0\}$ tais que

$$
\forall n \in \mathbb{N} \quad f_{n+1}<f_{n}<d_{0} \cdots d_{n}-\left(e_{0}+\cdots+e_{n}\right) .
$$


Isto pode ser feito, uma vez que $C$ não tem $(\omega, 1)$-aberturas.

Fixe $\left\{x_{n}: n \in \mathbb{N}\right\}$ uma enumeração para $h[A]$. Definimos $a_{0}=e_{0}+d_{x_{0}}+f_{0}, a_{n+1}=$ $a_{n}+\left(e_{n+1}+d_{x_{n+1}}+f_{n+1}\right), b_{0}=d_{0} \cdot\left(-e_{x_{0}}\right)$ e $b_{n+1}=b_{n} \cdot\left(d_{n+1} \cdot\left(-e_{x_{n+1}}\right)\right)$. Temos que

$$
\forall n \in \mathbb{N} \quad a_{n}<a_{n+1}<b_{n+1}<b_{n} .
$$

Como $C$ não tem $(\omega, \omega)$-aberturas, existe $c \in C$ tal que para todo $n \in \mathbb{N}, a_{n}<c<b_{n}$. Vejamos que tal $c$ satisfaz $(i),(i i),(i i i)$ :

(i) se $x \in A, x \leq b$, então $h(x) \in E$ e, portanto, $h(x) \leq c$. Analogamente, temos que se $x \in A, b \leq x$, então $c \leq h(x)$ e assim, temos $(i)$;

(ii) se $x \in A$ e $\forall d \in D, x \cdot d \neq 0$, então $0<d_{x}<c$ e daí, $d_{x} \leq c \cdot x$. Logo, $c$ satisfaz (ii);

(iii) análogo ao item (ii).

Proposição 6.14. Se $|A| \leq \omega_{1}$ e $B$ é uma álgebra sem $(\omega, \omega)$-aberturas nem $(\omega, 1)$ aberturas, então $B$ tem subálgebra isomorfa a A. Além disso, CH implica que $\wp(\mathbb{N}) / F$ in é a única álgebra de Boole de cardinalidade $2^{\omega}$ sem $(\omega, \omega)$-aberturas nem $(\omega, 1)$-aberturas.

Demonstração. Considere $A=\left(a_{\alpha}\right)_{\alpha<\omega_{1}}$. Definamos $\left(A_{\alpha}\right)_{\alpha<\omega_{1}}$, subálgebras de $A$ dadas por $A_{0}=\{0,1\}, A_{\alpha+1}=\left\langle A_{\alpha} \cup\left\{a_{\alpha}\right\}\right\rangle$ para todo $\alpha<\omega_{1}$ e se $\lambda<\omega_{1}$ é um ordinal limite, tome $A_{\lambda}=\bigcup_{\alpha<\lambda} A_{\alpha}$. Assim, temos $A=\bigcup_{\alpha<\omega_{1}} A_{\alpha}$.

Vamos construir, para cada $\alpha<\omega_{1}, h_{\alpha}: A_{\alpha} \rightarrow B$ um monomorfismo tal que se $\beta<\alpha$, então $\left.h_{\alpha}\right|_{A_{\beta}}=h_{\beta}$. Para isso, tome $h_{0}: A_{0} \rightarrow B$ dado por $h_{0}(0)=0$ e $h_{0}(1)=1$. Suponha então $\alpha<\omega_{1}$ e suponha que temos $h_{\beta}$ como acima, para todo $\beta<\alpha$. Daí, se $\alpha$ é limite, tome $h_{\alpha}=\bigcup_{\beta<\alpha} h_{\beta}$. Se $\alpha=\beta+1$, podemos estender $h_{\beta}$ para $h_{\alpha}$, como queremos, pela proposição anterior.

Portanto, $h=\bigcup_{\alpha<\omega_{1}} h_{\alpha}$ é um monomorfismo de $A$ em $B$.

Vejamos agora a segunda parte do resultado. Para isso, é suficiente mostrar que se $A$ e $B$ são álgebras de cardinalidade $\omega_{1} \operatorname{sem}(\omega, \omega)$-aberturas e sem $(\omega, 1)$-aberturas, então $A$ e $B$ são isomorfas. Sejam então álgebras $A$ e $B$ desta forma. 
Considere $\left(a_{\alpha}\right)_{\alpha<\omega_{1}}$ e $\left(b_{\alpha}\right)_{\alpha<\omega_{1}}$ enumerações das álgebras $A$ e $B$ respectivamente. Construímos, por indução, $A_{\alpha}$ subálgebra de $A, B_{\alpha}$ subálgebra de $B$ e $h_{\alpha}: A_{\alpha} \rightarrow B_{\alpha}$ isomorfismo de forma que $a_{\alpha} \in A_{\alpha+1}$ e $b_{\alpha} \in B_{\alpha+1}$.

Tomemos $A_{0}=\{0,1\}, B_{0}=\{0,1\}$ e $h_{0}: A_{0} \rightarrow B_{0}$ isomorfismo. Seja então $\alpha<\omega_{1}$ e suponhamos que temos, para cada $\beta<\alpha, h_{\beta}, A_{\beta}$ e $B_{\beta}$ como acima. Se $\alpha$ é um ordinal limite, tome $A_{\alpha}=\bigcup_{\beta<\alpha} A_{\beta}, B_{\alpha}=\bigcup_{\beta<\alpha} B_{\beta}$ e $h_{\alpha}=\bigcup_{\beta<\alpha} h_{\beta}$.

Se $\alpha=\beta+1$, podemos estender $h_{\beta}$ para um isomorfismo $g:\left\langle A_{\beta} \cup\left\{a_{\alpha}\right\}\right\rangle \rightarrow B_{\beta}^{\prime}$, pela proposição anterior. Daí, tome $B_{\alpha+1}=\left\langle B_{\beta}^{\prime} \cup\left\{b_{\alpha}\right\}\right\rangle$ e podemos estender $g^{-1}$ para um isomorfismo $g^{\prime}: B_{\alpha+1} \rightarrow A_{\alpha+1}$, pela proposição anterior.

Portanto, $h=\bigcup_{\alpha<\omega_{1}} h_{\alpha}$ é um isomorfismo de $A$ sobre $B$.

Proposição 6.15. $\omega^{*}$ não tem seqüências convergentes não triviais.

Demonstração. Como $\beta \mathbb{N}$ não tem seqüências convergentes não triviais e $\omega^{*}$ é subespaço fechado de $\beta \mathbb{N}$, é fácil ver que $\omega^{*}$ também não tem seqüências convergentes não triviais.

Convém lembrar que, como provamos que $l_{\infty} / c_{0}$ é um espaço de Grothendieck, segue do Teorema 3.30 e da Teorema 3.44 que $\omega^{*}$ não pode, de fato, ter seqüências convergentes não triviais.

Proposição 6.16. Se $X \subseteq \omega^{*}$ é homeomorfo a $\beta \mathbb{N}$, então $X$ é retrato de $\omega^{*}$.

Demonstração. Seja $h: \wp(\mathbb{N}) / F i n \rightarrow \wp(\mathbb{N})$ um epimorfismo. Considere $\left(a_{n}\right)_{n \in \mathbb{N}} \subseteq \wp(\mathbb{N})$ uma anticadeia tal que $\bigcup_{n \in \mathbb{N}} a_{n}=\mathbb{N}$ e $h\left(\left[a_{n}\right]\right)=\{n\}$. Seja então $A=\left\{\left[\bigcup_{n \in X} a_{n}\right]: X \in\right.$ $\wp(\mathbb{N})\}$. Vejamos que $A$ é uma subálgebra de $\wp(\mathbb{N}) / F i n$. Para isso, considere $X, Y \subseteq \mathbb{N}$ e temos:

- $\left[\bigcup_{n \in X} a_{n}\right] \cup\left[\bigcup_{n \in Y} a_{n}\right]=\left[\bigcup_{n \in X \cup Y} a_{n}\right]$;

- $\left[\bigcup_{n \in X} a_{n}\right] \cap\left[\bigcup_{n \in Y} a_{n}\right]=\left[\bigcup_{n \in X \cap Y} a_{n}\right]$, uma vez que $a_{n}$ 's são dois a dois disjuntos;

- $[\mathbb{N}] \backslash\left[\bigcup_{n \in X} a_{n}\right]=\left[\bigcup_{n \in \mathbb{N} \backslash X} a_{n}\right]$, uma vez que $a_{n}$ 's são dois a dois disjuntos e $\bigcup_{n \in \mathbb{N}} a_{n}=$ $\mathbb{N}$

- $[\mathbb{N}]=\left[\bigcup_{n \in \mathbb{N}} a_{n}\right]$ e $[\emptyset]=\left[\bigcup_{n \in \emptyset} a_{n}\right]$. 
Portanto, $A$ é subálgebra de $\wp(\mathbb{N}) / F i n$. Vejamos por fim que $\left.h\right|_{A}$ é isomorfismo de $A$ sobre $\wp(\mathbb{N})$. Já sabemos que é homomorfismo. Vejamos que se $X \in \wp(\mathbb{N})$, então $h\left(\left[\bigcup_{n \in X} a_{n}\right]\right)=$ $X$. Temos que se $k \in X$, então $\{k\}=h\left(\left[a_{k}\right]\right) \subseteq h\left(\left[\bigcup_{n \in X} a_{n}\right]\right)$ e se $k \notin X$, então $\{k\}=h\left(\left[a_{k}\right]\right) \subseteq h\left(\left[\bigcup_{n \in \mathbb{N} \backslash X}\right]\right)$. Daí, é fácil ver que $\left.h\right|_{A}$ é um isomorfismo de $A$ sobre $\wp(\mathbb{N})$.

Assim, temos que todo epimorfismo $h: \wp(\mathbb{N}) / F i n \rightarrow \wp(\mathbb{N})$ é um retrato. Em particular, pela Proposição 1.69 , temos que todo subespaço de $\omega^{*}$ homeomorfo a $\beta \mathbb{N}$ é um retrato.

Proposição 6.17. Existe $X \subseteq \omega^{*}$ homeomorfo a $\omega^{*}$ tal que $X$ não é retrato de $\omega^{*}$.

Demonstração. Seja $Y \subseteq \omega^{*}$ discreto e enumerável. Pela Proposição $5.14, \bar{Y} \subseteq \omega^{*}$ é homeomorfo a $\beta \mathbb{N}$. Considere $X=\bar{Y} \backslash \mathbb{N}_{\bar{Y}}$. Temos que $X$ é homeomorfo a $\omega^{*}$.

Suponhamos, por absurdo, que existe $f: \omega^{*} \rightarrow X$ uma retração. Daí, $\left.f\right|_{\bar{Y}}$ é uma retração de $\bar{Y}$ sobre $X$, contradizendo a Proposição 5.13 .

Proposição 6.18. (CH) Se $A$ é completa e $|A| \leq 2^{\omega}$, então existe $h: \wp(\mathbb{N}) / F i n \rightarrow A$ um retrato.

Demonstração. Como $|A| \leq 2^{\omega}=\omega_{1}$ e $\wp(\mathbb{N}) / F$ in não tem $(\omega, \omega)$-aberturas nem $(\omega, 1)$ aberturas, existe $h: A \rightarrow \wp(\mathbb{N}) / F$ in um monomorfismo. Tomando $C=h[A]$, temos que $h: A \rightarrow C$ é um isomorfismo e, portanto, $h^{-1}: C \rightarrow A$ é um isomorfismo. Como $A$ é completa, pelo Teorema de Extensão de Sikorski (1.28), existe $\tilde{h}: \wp(\mathbb{N}) / F i n \rightarrow A$ um homomorfismo que estende $h$. Tal $\tilde{h}$ é um retrato.

O próximo resultado é um resultado de consistência, uma vez que assumimos a hipótese do contínuo. Tal resultado foi provado por Negrepontis em [Ne]. Dow provou a consistência da negação do resultado abaixo, no modelo obtido com o forcing de Cohen (veja [Do1]).

Proposição 6.19. (CH) Se $\left(a_{n}\right)_{n \in \mathbb{N}} \subseteq \wp(\mathbb{N}) /$ Fin é uma anticadeia, então $\overline{\bigcup_{n \in \mathbb{N}} a_{n}^{*}}$ é um retrato de $\omega^{*}$.

Demonstração. Seja $X=\overline{\bigcup_{n \in \mathbb{N}} a_{n}^{*}}$. Considere $h: \wp(\mathbb{N}) / F i n \rightarrow C l o p(X)$ o epimorfismo dado por $h(a)=a^{*} \cap X$. Notemos que é suficiente obter $g: C l o p(X) \rightarrow \wp(\mathbb{N}) / F$ in um monomorfismo tal que $g\left(a_{n}^{*}\right)=a_{n}$ para todo $n \in \mathbb{N}$ e $\left\{a \in \wp(\mathbb{N}) / F\right.$ in $\left.: \exists n \in \mathbb{N} a \leq a_{n}\right\} \subseteq$ 
$g[C l o p(X)]$. Para isso, vejamos que $C=g[C l o p(X)]$ é tal que $\left.h\right|_{C}$ é um isomorfismo sobre $\operatorname{Clop}(X)$.

$\left.h\right|_{C}$ é injetor, pois se $b \in C l o p(X)$ e $h(g(b))=0$, então $g(b)^{*} \cap a_{n}^{*}=\emptyset$ para todo $n \in \mathbb{N}$. Daí, $g\left(b \cap a_{n}^{*}\right)=g(b) \cap a_{n}=\emptyset$ para todo $n \in \mathbb{N}$. Como $g$ é monomorfismo, segue que $b \cap a_{n}^{*}=\emptyset$ para todo $n \in \mathbb{N}$ e, portanto, $b=\emptyset$. Logo, $g(b)=\emptyset$.

$\left.h\right|_{C}$ é sobrejetor, pois dado $b \in C \operatorname{lop}(X)$, temos que $g\left(a_{n}^{*} \cap b\right)=a_{n} \cap g(b)<a_{n}$ para todo $n \in \mathbb{N}$. Como $\left\{a \in \wp(\mathbb{N}) /\right.$ Fin $\left.: \exists n \in \mathbb{N} a \leq a_{n}\right\} \subseteq g[\operatorname{Clop}(X)]$, temos que para cada $n \in N$, existe $b_{n} \in C l o p(X)$ tal que $g\left(b_{n}\right)=g\left(a_{n}^{*} \cap b\right)$. Então, para todo $n \in \mathbb{N}$,

$$
b_{1}+\cdots+b_{n}<b<\left(X \backslash\left(a_{1}^{*} \backslash b_{1}\right)\right) \cdots\left(X \backslash\left(a_{n}^{*} \backslash b_{n}\right)\right) .
$$

Logo, para todo $n \in \mathbb{N}$,

$$
g\left(b_{1}+\cdots+b_{n}\right)<g(b)<g\left(\left(X \backslash\left(a_{1}^{*} \backslash b_{1}\right)\right) \cdots\left(X \backslash\left(a_{n}^{*} \backslash b_{n}\right)\right)\right) .
$$

Daí, é fácil ver que $h(g(b))=b$. Vamos então construir $g$.

Para isso, fixemos $k \in \mathbb{N}$ e sejam

$$
\begin{gathered}
B_{k}=\wp(\mathbb{N}) / \text { Fin } \mid a_{k}=\left\{b \in \wp(\mathbb{N}) / \text { Fin }: b \leq a_{k}\right\}, \\
A_{k}=C \operatorname{lop}(X) \mid a_{k}^{*}=\left\{a \in C \operatorname{lop}(X): a \leq a_{k}^{*}\right\} .
\end{gathered}
$$

Vamos construir $g_{k}: A_{k} \rightarrow B_{k}$ um monomorfismo. Fixemos $A_{k}=\left\{a_{\alpha}: \alpha<2^{\omega}=\omega_{1}\right\}$. Construímos uma cadeia $\left(C_{\alpha}\right)_{\alpha<\omega_{1}} \subseteq A_{k}$ e $e_{\alpha}: C_{\alpha} \rightarrow B_{k}$ monomorfismos tais que

i) $\alpha<\beta<\omega_{1} \Rightarrow C_{\alpha} \subseteq C_{\beta}$;

ii) $\left.e_{\beta}\right|_{C_{\alpha}}=e_{\alpha}$;

iii) $a_{\alpha} \in C_{\alpha+1}$.

Daí, basta tomar $g_{k}=\bigcup_{\alpha<\omega_{1}} e_{\alpha}$ e temos o monomorfismo que queremos.

Para a construção, tomemos $C_{0}=\left\{\emptyset, a_{k}^{*}\right\}$ e $e_{0}(\emptyset)=0$ e $e_{0}\left(a_{k}^{*}\right)=a_{k}$. Seja $\alpha<\omega_{1}$ e suponha que temos $\left(C_{\beta}\right)_{\beta<\alpha}$ e $\left(e_{\beta}\right)_{\beta<\alpha}$ satisfazendo $\left.\left.\left.i\right), i i\right), i i i\right)$.

Caso 1: $\alpha$ é ordinal limite. 
Tomemos $C_{\alpha}=\bigcup_{\beta<\alpha} C_{\beta}$ e $e_{\alpha}=\bigcup_{\beta<\alpha} e_{\beta}$ e temos o que queremos.

Caso 2: $\alpha=\alpha^{\prime}+1$.

Tomemos $C_{\alpha}=\left\langle C_{\alpha^{\prime}} \cup\left\{a_{\alpha}\right\}\right\rangle$. Se $a_{\alpha} \in C_{\alpha^{\prime}}$, basta tomar $e_{\alpha}=e_{\alpha^{\prime}}$. Senão, como $\left|C_{\alpha^{\prime}}\right|<$ $\omega_{1}$ e $B_{k}$ é isomorfa a $\wp(\mathbb{N}) / F i n$, pela Proposição 6.13 temos que existe $e_{\alpha}: C_{\alpha} \rightarrow B_{k}$ um monomorfismo que estende $e_{\alpha^{\prime}}$. É fácil ver que temos $\left.\left.\left.i\right), i i\right), i i i\right)$.

Considere então $\phi: \bigcup_{n \in \mathbb{N}} A_{n} \rightarrow \wp(\mathbb{N}) / F i n$ dada por $\phi(a)=g_{k}(a)$ se $a \in A_{k}$. Pelo Primeiro Critério de Extensão de Sikorski (1.22), temos que existe $g:\left\langle\bigcup_{n \in \mathbb{N}} A_{n}\right\rangle \rightarrow$ $\wp(\mathbb{N}) / F$ in um monomorfismo tal que $g(a)=\phi(a)$ se $a \in \bigcup_{n \in \mathbb{N}} A_{n}$.

Por fim, como $\left|\left\langle\bigcup_{n \in \mathbb{N}} A_{n}\right\rangle\right| \leq \omega$, temos que, pela Proposição 6.13, existe $\tilde{g}: C l o p(X) \rightarrow$ $\wp(\mathbb{N}) / F$ in um monomorfismo que estende $g$, como queríamos.

\subsection{Subespaços complementados do $l_{\infty} / c_{0}$}

Mais uma vez, analogamente ao estudo do capítulo anterior para o $l_{\infty}$, fazemos aqui um estudo dos subespaços complementados do espaço $l_{\infty} / c_{0}$. Porém, os resultados aqui não são tão fortes como naquele caso.

Vejamos, por exemplo, que $l_{\infty} / c_{0}$ não é injetivo, como acontece no caso do $l_{\infty}$. Vimos que a álgebra $\wp(\mathbb{N}) / F i n$ não é completa, mas ainda assim poderíamos ter que $C(S(\wp(\mathbb{N}) / F$ in $)) \equiv l_{\infty} / c_{0}$ fosse injetivo.

Proposição 6.20. $l_{\infty} / c_{0}$ não é injetivo.

Demonstração. Faremos aqui apenas um esboço da demonstração, pois os argumentos aqui utilizados são análogos a argumentos que já apareceram. Pela Proposição 6.5, temos que existe $\left(a_{\alpha}\right)_{\alpha<2^{\omega}} \subseteq \wp(\mathbb{N}) /$ fin uma anticadeia. Daí, analogamente a demonstração da Proposição 3.28, podemos mostrar que $A=\left\langle\left\{a_{\alpha}: \alpha<2^{\omega}\right\}\right\rangle$ é isomorfa ${ }^{2}$ a FinCofin $\left(2^{\omega}\right)$. Logo, como na demonstração do Exemplo 3.27, temos que ${ }^{3} C(S(A)) \sim$ $C\left(S\left(\right.\right.$ FinCofin $\left.\left(2^{\omega}\right)\right) \sim c_{0}\left(2^{\omega}\right)$. Assim, temos uma imersão de $c_{0}\left(2^{\omega}\right)$ em $l_{\infty} / c_{0}$.

\footnotetext{
${ }^{2}$ FinCofin $\left(2^{\omega}\right)=\left\{X \subseteq 2^{\omega}:|X|<\infty\right.$ ou $\left.\left|2^{\omega} \backslash X\right|<\infty\right\}$.

${ }^{3} c_{0}\left(2^{\omega}\right)$ é o espaço das seqüências $\left(x_{\alpha}\right)_{\alpha<2^{\omega}}$ tais que para todo $\alpha<2^{\omega}, x_{\alpha} \in \mathbb{R}$ e para todo $\varepsilon>0$, $\left\{\alpha:\left|x_{\alpha}\right| \geq \varepsilon\right\}$ é finito.
} 
Tal imersão não pode ser estendida a $l_{\infty}\left[2^{\omega}\right]$ (as seqüências limitadas com índice em $\left.2^{\omega}\right)$, pois $d\left(l_{\infty}\left[2^{\omega}\right]\right)=\left|\wp\left(2^{\omega}\right)\right|=2^{2^{\omega}}>2^{\omega}=|\wp(\mathbb{N}) / F i n|=d\left(l_{\infty} / c_{0}\right)$.

Temos assim uma tradução do fato que $\wp(\mathbb{N}) / F i n$ não é completa para o fato que $l_{\infty} / c_{0}$ não é injetivo. Porém, não se sabe se esta tradução vale sempre, isto é, se o fato que uma álgebra de Boole $A$ não é completa implica que $C(S(A))$ não é injetivo.

O próximo resultado que temos é interessante, uma vez que não há em $\omega^{*}$ nenhum aberto-fechado homeomorfo a $\beta \mathbb{N}$, mas ainda assim temos uma cópia de $l_{\infty}$ complementada $\mathrm{em} l_{\infty} / c_{0}$ :

Teorema 6.21. $l_{\infty} / c_{0} \sim l_{\infty} \oplus l_{\infty} / c_{0}$.

Demonstração. Sabemos, pela Proposição 6.16, que $\beta \mathbb{N}$ é retrato de $\omega^{*}$. Então, do Exemplo 3.22 , temos que $l_{\infty}$ é complementado em $l_{\infty} / c_{0}$. Seja $A$ espaço de Banach tal que $l_{\infty} / c_{0} \sim l_{\infty} \oplus A$. Daí:

$$
l_{\infty} / c_{0} \sim l_{\infty} \oplus A \sim l_{\infty} \oplus l_{\infty} \oplus A \sim l_{\infty} \oplus l_{\infty} / c_{0}
$$

Queremos agora fortalecer tal resultado. Ainda não se sabe se isso é possível, sem assumir nenhuma hipótese adicional. Sabemos apenas que, assumindo a hipótese do contínuo, podemos fortalecê-lo um pouco. Para entender isto, vejamos primeiramente o seguinte:

Lema 6.22. Se $\phi: \wp(\mathbb{N}) / F i n \backslash\{0\} \rightarrow \mathbb{R}$ é tal que $a \leq b \Rightarrow \phi(a) \leq \phi(b)$, então para todo $a \in \wp(\mathbb{N}) / F$ in $\backslash\{0\}$, existe $b \in \wp(\mathbb{N}) / F$ in $\backslash\{0\}$ tal que $b \leq a$ e $\phi$ é constante abaixo de $b$.

Demonstração. Suponhamos, por absurdo, que o resultado não vale. Daí, existe $a \in$ $\wp(\mathbb{N}) / F$ in $\backslash\{0\}$ tal que para todo $0<b<a$, existe $0<c<b$ tal que $\phi(c)<\phi(b)$. Como podemos construir cadeias decrescentes não enumeráveis em $\wp(\mathbb{N}) / F i n$, obteríamos uma seqüência decrescente não enumerável de reais, o que é impossível, uma vez que neste caso teríamos não enumeráveis intervalos dois a dois disjuntos na reta real.

Lema 6.23. Seja $A$ uma álgebra de Boole e $K$ seu espaço de Stone. Se $T: C(K) \rightarrow C(K)$ é um operador não negativo, isto é, $T\left(\chi_{a^{*}}\right)(x) \geq 0$ para todo $x \in K$ e todo a $\in A$, então $T$ é monótono, isto é, $a \leq b \Rightarrow T\left(\chi_{a^{*}}\right) \leq T\left(\chi_{b^{*}}\right)$. 
Demonstração. Suponhamos, por absurdo, que o resultado não vale. Então, existem $a \leq b$ e $x \in X$ tais que $T\left(\chi_{a^{*}}\right)(x)>T\left(\chi_{b^{*}}\right)(x)$. Mas daí,

$$
T\left(\chi_{(b-a)^{*}}\right)(x)=T\left(\chi_{b^{*}}-\chi_{a^{*}}\right)(x)=T\left(\chi_{b^{*}}\right)(x)-T\left(\chi_{a^{*}}\right)(x)<0,
$$

contradizendo a hipótese.

Lema 6.24. Seja A uma álgebra de Boole e $K$ seu espaço de Stone. Se $T: C(K) \rightarrow C(K)$ é tal que existem $\alpha \leq 0 \leq \beta$ satisfazendo:

(a) $\alpha \leq T\left(\chi_{a^{*}}\right) \leq \beta$ para todo $a \in A$;

(b) para todo $\varepsilon>0$ e todo $a \in A \backslash\{0\}$, existe $b \leq$ a tal que $T\left(\chi_{b^{*}}\right)(x)>\beta-\varepsilon$ para algum $x \in a^{*}$;

(c) para todo $\varepsilon>0$ e todo $a \in A \backslash\{0\}$ existe $b \leq$ a tal que $T\left(\chi_{b^{*}}\right)(x)<\alpha+\varepsilon$ para algum $x \in a^{*}$.

Então temos que $T$ ou $-T$ é não negativo e existe $\gamma \in \mathbb{R}$ tal que

$$
T\left(\chi_{a^{*}}\right)=\gamma \cdot \chi_{a^{*}}
$$

para todo a $\in A$ e, conseqüentemente, $T(f)=\gamma \cdot f$ para todo $f \in C(K)$.

Demonstração. Para obter que $T$ ou $-T$ é não negativo, por $(a)$, basta mostrar que $\alpha=0$ ou $\beta=0$. Para isso, suponha que $\beta>0$ e $\alpha<0$. Tomemos $\varepsilon>0$ tal que $\varepsilon<\max \left\{\frac{-\alpha}{2}, \frac{\beta}{2}\right\}$. Daí, por $(c)$, existe $a \leq 1$ e $x \in K$ tal que

$$
T\left(\chi_{a^{*}}\right)(x)<\alpha+\varepsilon .
$$

Se $x \in a^{*}$, como $T\left(\chi_{a^{*}}\right) \in C(K)$, existe $b \leq a$ tal que

$$
\left.T\left(\chi_{a^{*}}\right)\right|_{b^{*}}<\alpha+\varepsilon
$$

Por (b), existe $c \leq b$ e $y \in b^{*}$ tal que

$$
T\left(\chi_{c^{*}}\right)(y)>\beta-\varepsilon .
$$

Novamente, como $T\left(\chi_{c^{*}}\right) \in C(K)$, existe $d \leq c$ tal que

$$
\left.T\left(\chi_{c^{*}}\right)\right|_{d^{*}}>\beta-\varepsilon
$$


Logo, temos

$$
\left.T\left(\chi_{(a-c)^{*}}\right)\right|_{d^{*}}=\left.T\left(\chi_{a^{*}}\right)\right|_{d^{*}}-\left.T\left(\chi_{c^{*}}\right)\right|_{d^{*}}<\alpha+\varepsilon-\beta+\varepsilon<\alpha,
$$

contradizendo (a).

Analogamente, podemos obter que se $x \in(-a)^{*}$, como $T\left(\chi_{a^{*}}\right) \in C(K)$, existe $b \leq-a$ tal que

$$
\left.T\left(\chi_{a^{*}}\right)\right|_{b^{*}}<\alpha+\varepsilon .
$$

Por (b), existe $c \leq b$ e $y \in b^{*}$ tal que

$$
T\left(\chi_{c^{*}}\right)(y)>\beta-\varepsilon .
$$

Novamente, como $T\left(\chi_{c^{*}}\right) \in C(K)$, existe $d \leq c$ tal que

$$
\left.T\left(\chi_{c^{*}}\right)\right|_{d^{*}}>\beta-\varepsilon .
$$

Logo, temos

$$
\left.T\left(\chi_{(a-c)^{*}}\right)\right|_{d^{*}}=\left.T\left(\chi_{a^{*}}\right)\right|_{d^{*}}-\left.T\left(\chi_{c^{*}}\right)\right|_{d^{*}}<\alpha+\varepsilon-\beta+\varepsilon<\alpha,
$$

contradizendo (a).

Assim, ou $\alpha=0 \mathrm{e}$, portanto, $T$ é não negativo, ou $\beta=0 \mathrm{e}$, portanto, $-T$ é não negativo.

Pelo lema anterior, temos que ou $T$ ou $-T$ é monótono. Suponha, sem perda de generalidade, que $T$ é monótono. Primeiramente, note que para todo $a \in A,\left.T\left(\chi_{a^{*}}\right)\right|_{(-a)^{*}}=$ 0 , pois se existisse $x \in(-a)^{*}$ tal que $T\left(\chi_{a^{*}}\right)(x)>0$, existiria $\varepsilon>0$ e $b \leq-a$ tal que $\left.T\left(\chi_{a^{*}}\right)\right|_{b^{*}}>\varepsilon$. Daí, tomando $d \leq c \leq b$ tal que $\left.T\left(\chi_{c^{*}}\right)\right|_{d^{*}}>\beta-\varepsilon$, teríamos que

$$
\left.T\left(\chi_{(a+c)^{*}}\right)\right|_{b^{*}}=\left.T\left(\chi_{a^{*}}\right)\right|_{b^{*}}+\left.T\left(\chi_{c^{*}}\right)\right|_{b^{*}}>\varepsilon+\beta-\varepsilon=\beta,
$$

contradizendo $(a)$.

Vejamos então que para todo $x \in a^{*}, T\left(\chi_{a^{*}}\right)(x)=\beta$. Para isso, suponha que para algum $x \in a^{*}$ tem-se que $T\left(\chi_{a^{*}}\right)(x)<\beta$. Tome $b \leq a$ e $\varepsilon>0$ tal que $\left.T\left(\chi_{a^{*}}\right)\right|_{b^{*}}<\beta-\varepsilon$. Seja então $c \leq b$ e $y \in b^{*}$ tais que $T\left(\chi_{c^{*}}\right)(y)>\beta-\frac{\varepsilon}{2}$. Daí,

$$
T\left(\chi_{(a-c)}^{*}\right)(y)=T\left(\chi_{a^{*}}\right)(y)-T\left(\chi_{c^{*}}\right)(y)<\beta-\varepsilon-\left(\beta-\frac{\varepsilon}{2}\right)<0,
$$


contradizendo $(a)$. Portanto, temos que $T\left(\chi_{a^{*}}\right)=\gamma \cdot \chi_{a^{*}}$, para $\gamma=\beta$, como queríamos.

Usando o Teorema de Stone-Weierstrass (3.2), temos que $T(f)=\gamma \cdot f$ para toda $f \in C(K)$.

Proposição 6.25. Seja $T: C\left(\omega^{*}\right) \rightarrow C\left(\omega^{*}\right)$ um operador. Então, para todo a $\in$ $\wp(\mathbb{N}) / F$ in $\backslash\{0\}$, existe $b \in \wp(\mathbb{N}) / F$ in $\backslash\{0\}$ com $b \leq a$ e $\gamma \in \mathbb{R}$ uma constante tais que

$$
\forall c \leq\left. b \quad T\left(\chi_{c^{*}}\right)\right|_{b^{*}}=\gamma \cdot \chi_{c^{*}}
$$

Demonstração. Para $a \in \wp(\mathbb{N}) / F i n \backslash\{0\}$, definimos

$$
\begin{gathered}
\phi(a)=\sup _{0 \leq b \leq a} \max _{x \in a^{*}} T\left(\chi_{b^{*}}\right)(x), \\
\psi(a)=-\inf _{0 \leq b \leq a} \min _{x \in a^{*}} T\left(\chi_{b^{*}}\right)(x) .
\end{gathered}
$$

Note que se $0<a \leq b$, então $0 \leq \phi(a) \leq \phi(b)$ e $0 \leq \psi(a) \leq \psi(b)$. Assim, dado $a \in \wp(\mathbb{N}) / F i n$, aplicando o Lema 6.22 duas vezes, obtemos $b \in \wp(\mathbb{N}) / F i n \backslash\{0\}$ e $\alpha \leq 0 \leq \beta$ tais que

$$
\forall c \leq b \quad \phi(c)=\beta \quad \text { e } \quad \psi(c)=\alpha .
$$

Logo, temos as hipóteses (a), (b), e (c) do lema anterior satisfeitas e, portanto, existe $\gamma \in \mathbb{R}$ tal que

$$
\forall c \leq b \quad T\left(\chi_{c^{*}}\right)=\gamma \cdot \chi_{c^{*}}
$$

Teorema 6.26. Sejam $A, B$ espaços de Banach tais que $C\left(\omega^{*}\right) \sim A \oplus B$. Então, ou $A$ ou $B$ contém uma cópia complementada de $C\left(\omega^{*}\right)$.

Demonstração. Considere $P_{1}, P_{2}$ as projeções de $C\left(\omega^{*}\right)$ sobre $A$ e $B$, respectivamente, tais que $I d=P_{1}+P_{2}$.

Seja $a_{1} \in \wp(\mathbb{N}) / F$ in $\backslash\{0\}$ e $\gamma_{1} \in \mathbb{R}$ tais que para todo $b \leq a_{1},\left.P_{1}\left(\chi_{b^{*}}\right)\right|_{a_{1}^{*}}=\gamma_{1} \chi_{b^{*}}$.

Seja então $a_{2} \leq a_{1}, a_{2} \in \wp(\mathbb{N}) / F$ in $\backslash\{0\}$ e $\gamma_{2} \in \mathbb{R}$ tais que para todo $b \leq a_{2}$, $\left.P_{2}\left(\chi_{b^{*}}\right)\right|_{a_{2}^{*}}=\gamma_{2} \chi_{b^{*}}$. 
Como $P_{1}+P_{2}=I d$, temos que ou $\gamma_{1} \neq 0$ ou $\gamma_{2} \neq 0$. Suponhamos, sem perda de generalidade, que $\gamma_{1} \neq 0$. Considere

$$
X=\left\{f \in C\left(\omega^{*}\right): \operatorname{supp}(f) \subseteq a_{2}^{*}\right\} .
$$

Temos que $P_{1}[X] \subseteq \operatorname{Im}\left(P_{1}\right)=A$. Vejamos que $P_{1}[X] \sim C\left(\omega^{*}\right)$ e que $P_{1}[X]$ é complementado em $A$. É fácil ver que $\left.P_{1}\right|_{X}$ é uma bijeção entre $X$ e $P_{1}[X]$. Além disso, dado $f \in X$, temos que

$$
\left|\gamma_{1}\right|\|f\| \leq\left\|P_{1}(f)\right\| \leq\left\|P_{1}\right\| \cdot\|f\| .
$$

Portanto, $\left.P_{1}\right|_{X}$ é um isomorfismo entre $X$ e $P_{1}[X]$. Logo,

$$
P_{1}[X] \sim X \sim C\left(a_{2}^{*}\right) \sim C\left(\omega^{*}\right)
$$

Defina $P: C\left(\omega^{*}\right) \rightarrow P_{1}[X]$, por $P(f)=\frac{1}{\gamma_{1}} P_{1}\left(\left.f\right|_{a_{2}^{*}}\right)$. É fácil ver que $P$ está bem definida e é linear. $P$ é contínua, pois dada $f \in C\left(\omega^{*}\right)$, temos

$$
\|P(f)\|=\left\|\frac{1}{\gamma_{1}} P_{1}\left(f_{a_{2}^{*}}\right)\right\| \leq \frac{1}{\left|\gamma_{1}\right|}\left\|P_{1}\right\|\left\|\left.f\right|_{a_{2}^{*}}\right\| \leq \frac{1}{\left|\gamma_{1}\right|}\left\|P_{1}\right\|\|f\| .
$$

Vejamos agora que $\left.P\right|_{P_{1}[X]}=I d$. Para isso, tomemos $f \in P_{1}[X]$. Daí, existe $g \in C\left(\omega^{*}\right)$ tal que $\operatorname{supp}(g) \subseteq a_{2}^{*}$ e $P_{1}(g)=f$. Assim,

$$
P(f)=P\left(P_{1}(g)\right)=\frac{1}{\gamma_{1}} P_{1}\left(\left.P_{1}(g)\right|_{a_{2}^{*}}\right)=\frac{1}{\gamma_{1}} P_{1}\left(\gamma_{1} g\right)=P_{1}(g)=f .
$$

Logo, $P$ é sobrejetora e $P^{2}=P$ e, portanto, $P$ é projeção de $C\left(\omega^{*}\right)$ sobre $P_{1}[X]$. Como $P_{1}[X] \subseteq A$, temos que $\left.P\right|_{A}$ é projeção de $A$ sobre $P_{1}[X]$. Daí, $P_{1}[X]$ é complementado em $A$.

Teorema 6.27. Seja $\left(a_{n}\right)_{n \in \mathbb{N}} \subseteq \wp(\mathbb{N}) /$ Fin uma anticadeia. Se $X=l_{\infty} / c_{0}$, então

$$
l_{\infty}(X) \equiv C\left(\overline{\bigcup_{n \in \mathbb{N}} a_{n}^{*}}\right) .
$$

Demonstração. Seja $\left(a_{n}\right)_{n \in \mathbb{N}} \subseteq \wp(\mathbb{N}) / F i n$ uma anticadeia e considere $K=\overline{\bigcup_{n \in \mathbb{N}} a_{n}^{*}}$.

Para cada $n \in \mathbb{N}$, temos que $C\left(a_{n}^{*}\right)$ é isométrico a $C\left(\omega^{*}\right) \equiv l_{\infty} / c_{0}$. Seja $T_{n}$ : $C\left(a_{n}^{*}\right) \rightarrow C\left(\omega^{*}\right)$ uma isometria. Seja $X=C\left(\omega^{*}\right)$ e defina $T: C(K) \rightarrow l_{\infty}(X)$ por 
$T(f)=\left(T_{n}\left(\left.f\right|_{a_{n}^{*}}\right)\right)_{n \in \mathbb{N}} . T$ está bem definida, pois dada $f \in C(K)$, temos que, para cada $n \in \mathbb{N}$,

$$
\left\|T_{n}\left(\left.f\right|_{a_{n}^{*}}\right)\right\|=\left\|\left.f\right|_{a_{n}^{*}}\right\| \leq\|f\| .
$$

Logo, $\sup _{n \in \mathbb{N}}\left\|T_{n}\left(\left.f\right|_{a_{n}^{*}}\right)\right\| \leq\|f\|<\infty$. Vejamos que $T$ é uma isometria.

$T$ é linear, pois é linear coordenada a coordenada. $T$ é injetora, pois se $f \in C(K)$ é tal que $T(f)=0$, então para todo $n \in \mathbb{N}, T_{n}\left(\left.f\right|_{a_{n}^{*}}\right)=0$. Como cada $T_{n}$ é isometria, temos que para todo $n \in \mathbb{N},\left.f\right|_{a_{n}^{*}}=0$. Logo, $\left.f\right|_{\cup_{n \in \mathbb{N}} a_{n}^{*}}=0$ e, como $f$ é contínua em $K$, segue que $f=0$.

Para mostrar que $T$ é sobrejetora, considere $\left(f_{n}\right)_{n \in \mathbb{N}} \in l_{\infty}(X)$. Para cada $n \in \mathbb{N}$, defina $g_{n}=T_{n}^{-1}\left(f_{n}\right) \in C\left(a_{n}^{*}\right)$ e considere $b_{n} \in \wp(\mathbb{N})$ tal que $a_{n}=\left[b_{n}\right]$, de forma que $\left(b_{n}\right)_{n \in \mathbb{N}}$ seja uma anticadeia. Temos que $a_{n}^{*}$ é um subespaço fechado de $b_{n}^{*}$. Assim, pelo Teorema de Extensão de Tietze (1.36), podemos estender cada $g_{n}$ para $\tilde{g}_{n} \in C\left(b_{n}^{*}\right)$, com $\left\|\tilde{g}_{n}\right\|=\|g\|$.

Considere $g \in l_{\infty}$ dada por $g(n)=\tilde{g}_{k}(n)$, se $n \in b_{k}^{*}$ e $g(n)=0$, caso contrário. $g$ está bem definida, pois $\left(b_{n}\right)_{n \in \mathbb{N}}$ é uma anticadeia e $\left(\tilde{g}_{n}\right)_{n \in \mathbb{N}}$ é limitada (uma vez que $\left(f_{n}\right)_{n \in \mathbb{N}}$ é limitada). Assim, $g \in l_{\infty}$ e, portanto, existe $h \in C(\beta \mathbb{N})$ tal que $\left.h\right|_{\mathbb{N}}=g$. Considere $\left.h\right|_{K} \in C(K)$ e temos que $T\left(\left.h\right|_{K}\right)=\left(f_{n}\right)_{n \in \mathbb{N}}$.

Por fim, se $f \in C(K)$, temos

$$
\begin{aligned}
\|T(f)\| & =\sup _{n \in \mathbb{N}}\left\|T_{n}\left(\left.f\right|_{a_{n}^{*}}\right)\right\|=\sup _{n \in \mathbb{N}}\left\|\left.f\right|_{a_{n}^{*}}\right\|=\sup _{n \in \mathbb{N}}\left(\sup _{x \in a_{n}^{*}}|f(x)|\right) \\
& =\sup _{x \in \cup_{n \in \mathbb{N}} a_{n}^{*}}|f(x)|=\sup _{x \in K}|f(x)|=\|f\|,
\end{aligned}
$$

pois $\bigcup_{n \in \mathbb{N}} a_{n}^{*}$ é denso em $K$. Portanto, $T$ é uma isometria, como queríamos.

O próximo teorema é, novamente, um resultado de consistência, uma vez que assumimos a hipótese do contínuo. Usaremos, para prová-lo, a Proposição 6.19, resultado de Negrepontis em [Ne]. Lembramos que o resultado daquela proposição é independente, sendo que a consistência de sua negação foi provada por Dow em [Do1]. O próximo resultado, que foi mostrado por Drewnowski e Roberts em [DR], garante que, assumindo $\mathrm{CH}$, temos que $l_{\infty} / c_{0}$ é primário ${ }^{4}$ :

Definição 6.28. Seja $X$ um espaço de Banach. Dizemos que $X$ é primário se, para toda decomposição $X \sim A \oplus B$, tem-se que ou $A \sim X$ ou $B \sim X$.

\footnotetext{
${ }^{4}$ Lembramos que, como já mencionado, esta propriedade é mais fraca que a propriedade de ser primo.
} 
Vejamos, enfim, o resultado.

Teorema 6.29 ([DR]). (CH) Se A,B são espaços de Banach e $l_{\infty} / c_{0} \sim A \oplus B$, então ou $A \sim l_{\infty} / c_{0}$ ou $B \sim l_{\infty} / c_{0}$.

Demonstração. Assumindo CH, temos, pela Proposição 6.19, que se $\left(a_{n}\right)_{n \in \mathbb{N}} \subseteq \wp(\mathbb{N}) / F$ in é uma anticadeia, então $\overline{\bigcup_{n \in \mathbb{N}} a_{n}^{*}}$ é retrato de $\omega^{*}$. Daí, pelo Exemplo 3.22 , temos que $C\left(\overline{\bigcup_{n \in \mathbb{N}} a_{n}^{*}}\right)$ é complementado em $C\left(\omega^{*}\right)$. Além disso, pelo Teorema 6.27 , temos que $C\left(\overline{\bigcup_{n \in \mathbb{N}} a_{n}^{*}}\right) \sim l_{\infty}(X)$, para $X=l_{\infty} / c_{0}$. Logo, existe um espaço de Banach $Y$ tal que

$$
\begin{aligned}
l_{\infty} / c_{0} & =C\left(\omega^{*}\right) \sim C\left(\overline{\bigcup_{n \in \mathbb{N}} a_{n}^{*}}\right) \oplus Y \sim l_{\infty}(X) \oplus Y \\
& \sim l_{\infty}(X) \oplus l_{\infty}(X) \oplus Y \sim l_{\infty}(X) \oplus l_{\infty} / c_{0} \sim l_{\infty}(X) .
\end{aligned}
$$

Suponha então $A, B$ espaços de Banach tais que $l_{\infty} / c_{0} \sim A \oplus B$. Logo, ou $A$ ou $B$ possui uma cópia complementada de $l_{\infty} / c_{0}$. Suponhamos, sem perda de generalidade, que $A$ possui uma cópia complementada de $l_{\infty} / c_{0}$. Seja $Z$ espaço de Banach tal que $A \sim l_{\infty} / c_{0} \oplus Z$. Temos

$$
\begin{aligned}
A & \sim l_{\infty} / c_{0} \oplus Z \sim l_{\infty} / c_{0} \oplus l_{\infty} / c_{0} \oplus Z \sim l_{\infty} / c_{0} \oplus A \\
& \sim l_{\infty}(X) \oplus A \sim l_{\infty}(A \oplus B) \oplus A \sim l_{\infty}(A) \oplus l_{\infty}(B) \oplus A \\
& \sim l_{\infty}(A) \oplus l_{\infty}(B) \sim l_{\infty}(A \oplus B) \sim l_{\infty}(X) \sim l_{\infty} / c_{0} .
\end{aligned}
$$

Convém mencionar que não se sabe se é consistente que existem $A, B$ não isomorfos a $l_{\infty} / c_{0}$ tais que $A \oplus B \sim l_{\infty} / c_{0}$. 


\section{Capítulo 7}

\section{Pequenos espaços de Grothendieck $C(K)$}

O objetivo deste capítulo é apresentar resultados sobre certos exemplos de álgebras de Boole ou espaços da forma $C(K)$ com a propriedade de Grothendieck. Mais especificamente, faremos dois tipos de construções de álgebras de Boole: por indução transfinita e usando forcing.

Baseado em exemplos clássicos $\left(l_{\infty}\right.$ e $\left.l_{\infty} / c_{0}\right)$ e no Teorema de Sobczyk, Pełczyński perguntou se todo espaço da forma $C(K)$ tem, ou uma cópia complementada de $c_{0}$, ou uma cópia (complementada) de $l_{\infty}$. Haydon mostrou em [Ha] o seguinte resultado, que responde negativamente esta pergunta:

Teorema. Existe uma álgebra de Boole infinita $A$ tal que, sendo $S(A)$ seu espaço de Stone, $C(S(A))$ é um espaço de Banach sem cópias complementadas de $c_{0}$ nem de $l_{\infty}$.

Meu objetivo na primeira seção deste capítulo é mostrar este teorema. Para isso, constrói-se, por indução transfinita, uma álgebra de Boole $A$ de subconjuntos de $\mathbb{N}$ que contém todos os conjuntos finitos, tem a propriedade de completude subseqüencial e tal que para nenhum $X \subseteq \mathbb{N}$ infinito tem-se que $\wp(X)=\{X \cap a: a \in A\}$. O espaço $C(K)$, onde $K$ é o espaço de Stone desta álgebra, é o espaço com as propriedades que queremos. A propriedade de completude subseqüencial garante que $C(K)$ não possui cópias complementadas de $c_{0}$ e que tem a propriedade de Grothendieck. Para mostrar que $C(K)$ não possui cópias de $l_{\infty}$, usamos as demais propriedades da álgebra. 
Tendo em vista os principais exemplos de espaços de Grothendieck, perguntamos em que sentido um espaço de Grothendieck da forma $C(K)$ deve ser grande. Pelo resultado acima, tais espaços não têm necessariamente um subespaço isomorfo ao $l_{\infty}$. Podemos formular então a seguinte pergunta: será que todo espaço da forma $C(K)$ de Grothendieck tem $l_{\infty}$ como quociente? Um exemplo construído por Talagrand em 1980 (veja [Ta]), assumindo $\mathrm{CH}$, mostra que não. Por outro lado, Haydon, Levy e Odell mostraram (veja [HLO]) que assumindo o axioma de Martin (MA) e a negação da hipótese do contínuo $(\neg \mathrm{CH})$ a resposta para esta pergunta é sim. Logo, a resposta para tal pergunta depende de hipóteses conjuntísticas adicionais. Entramos assim em questões independentes relativas aos espaços de Grothendieck.

Que outras interpretações podemos então dar para "grande", e perguntar se espaços de Grothendieck $C(K)$ podem ser pequenos? Podemos olhar para o espaço topológico $K$ e tentar caracterizar $K$ tal que $C(K)$ é Grothendieck. Por exemplo, pelo Teorema 3.44, sabemos que se $K$ é booleano e $C(K)$ é um espaço de Grothendieck, então $C(K)$ não tem cópias complementadas de $c_{0}$ e, portanto, pelo Teorema $3.30, K$ não tem seqüências convergentes não triviais. Porém, isto não é suficiente para que $C(K)$ seja Grothendieck, como ilustra o Exemplo 4.10 de [Scha].

Podemos dar outra interpretação para $K$ "grande": ter peso grande (por exemplo, pelo menos $2^{\omega}$ ). Podemos também tentar determinar os possíveis pesos de $K$ de forma que $C(K)$ seja Grothendieck. Vimos que se $K$ é booleano, o peso de $K$ é a cardinalidade de $C l o p(K)$ e é, também, a densidade de $C(K)$.

O objetivo da Seção 7.2 é apresentar dois teoremas que, combinados, fornecem a independência da existência de espaços booleanos $K$ de peso menor que $2^{\omega}$ tal que $C(K)$ é Grothendieck . O primeiro deles, é um resultado conhecido:

Teorema. (MA) Se K é um espaço booleano infinito com peso menor que $2^{\omega}$, então $C(K)$ possui uma cópia complementada de $c_{0}$ e, portanto, não é Grothendieck.

O resultado que fornece a consistência da negação da afirmação acima, será provado usando o forcing de Sacks, já apresentado no Capítulo 4:

Teorema. $\mathrm{Na}$ extensão obtida com o produto de $\omega_{2}$ forcing's de Sacks, existe uma álgebra de Boole $A$ de cardinalidade $\omega_{1}<2^{\omega}$ tal que $C(S(A))$ é um espaço de Grothendieck. 
Tal resultado encontra-se em [Bre] e foi motivado pelo artigo [JK], onde mostra-se que na extensão obtida com o forcing de Sacks, existe uma álgebra de Boole $A$ com cardinalidade menor que $2^{\omega}$ e tal que $S(A)$ não tem seqüências convergentes não triviais. A demonstração deste resultado foi modificada e generalizada, de forma a obter o teorema acima enunciado.

Veremos adiante que, como a álgebra construída $A$ é tal que $|A|<2^{\omega}, A$ não tem diversas propriedades ${ }^{1}$ de álgebras de Boole consideradas na literatura que implicam que $C(S(A))$ tem a propriedade de Grothendieck, uma vez que tais propriedades implicam também que a álgebra tenha pelo menos $2^{\omega}$ elementos.

Como referência para os assuntos aqui tratados, indicamos [Ha] para Seção 7.1 e [Bre] para a Seção 7.2 .

\subsection{A álgebra de Haydon}

Como dissemos na introdução, o objetivo desta seção é fazer a construção de uma álgebra de Boole com certas propriedades, que implicarão que $C(K)$, onde $K$ é o espaço de Stone desta álgebra, não tem cópias complementadas de $c_{0}$ nem cópias, necessariamente complementadas, de $l_{\infty}$.

Vamos então construir a álgebra que queremos. Tal construção será feita por indução e a álgebra obtida é uma subálgebra de $\wp(\mathbb{N})$ com boas propriedades. Para o passo indutivo, precisamos do seguinte lema:

Lema 7.1. Seja $\omega \leq \gamma<2^{\omega}$ um ordinal e seja A uma subálgebra de Boole de $\wp(\mathbb{N})$ com $|A| \leq|\gamma|$. Suponha que $\left(M_{\beta}, N_{\beta}\right)_{\beta<\gamma}$ é uma família de pares de subconjuntos de $\mathbb{N}$ com $M_{\beta} \subseteq N_{\beta}$ para todo $\beta<\gamma e$

$$
\forall a \in A \quad \forall \beta<\gamma \quad M_{\beta} \neq N_{\beta} \cap a
$$

Então, dada uma anticadeia $\left(a_{n}\right)_{n \in \mathbb{N}} \subseteq A$, existe $\rho \subseteq \mathbb{N}$ infinito tal que se $A_{\rho}$ é a álgebra gerada por $A$ e $a_{\rho}=\bigcup_{n \in \rho} a_{n}=\sum_{n \in \rho} a_{n}$, então

$$
\forall a \in A_{\rho} \quad \forall \beta<\gamma \quad M_{\beta} \neq N_{\beta} \cap a .
$$

\footnotetext{
${ }^{1} \mathrm{~A}$ PCS é uma destas propriedades e podemos encontrar outras em [Ai].
} 
Demonstração. Seja $\left(X_{\alpha}\right)_{\alpha<2^{\omega}}$ uma família quase disjunta de subconjuntos de $\mathbb{N}$ e suponhamos por absurdo que para todo $\alpha<2^{\omega}$, se $\rho=X_{\alpha}$ o lema não vale. Assim, para cada $\alpha<2^{\omega}$, existem, pelo Lema $1.10, E_{\alpha}, F_{\alpha}, G_{\alpha} \in A$ disjuntos e $\beta_{\alpha}<\gamma$ tais que

$$
N_{\beta_{\alpha}} \cap\left(E_{\alpha} \cup\left(F_{\alpha} \cap a_{X_{\alpha}}\right) \cup\left(G_{\alpha} \backslash a_{X_{\alpha}}\right)\right)=M_{\beta_{\alpha}}
$$

Como existem apenas $|\gamma| \cdot|\gamma| \cdot|\gamma| \cdot|\gamma|=|\gamma|<2^{\omega}$ possibilidades para $\left(E_{\alpha}, F_{\alpha}, G_{\alpha}, \beta_{\alpha}\right)$, existem $\alpha_{1}, \alpha_{2}<2^{\omega}$ distintos, $E, F, G \in A$ e $\beta<\gamma$ tais que $(E, F, G, \beta)=\left(E_{\alpha_{i}}, F_{\alpha_{i}}, G_{\alpha_{i}}, \beta_{\alpha_{i}}\right)$, para $i=1,2$. Em particular,

$$
\begin{aligned}
N_{\beta} \cap\left(F \cap a_{X_{\alpha_{1}}}\right) & =M_{\beta} \cap F=N_{\beta} \cap\left(F \cap a_{X_{\alpha_{2}}}\right), \\
N_{\beta} \cap\left(G \backslash a_{X_{\alpha_{1}}}\right) & =M_{\beta} \cap G=N_{\beta} \cap\left(G \backslash a_{X_{\alpha_{2}}}\right) .
\end{aligned}
$$

Logo, temos que

$$
\begin{gathered}
M_{\beta} \cap F=N_{\beta} \cap\left(F \cap\left(a_{X_{\alpha_{1}}} \cap a_{X_{\alpha_{2}}}\right)\right), \\
M_{\beta} \cap G=N_{\beta} \cap\left(G \backslash\left(a_{X_{\alpha_{1}} \cap X_{\alpha_{2}}}\right)\right) .
\end{gathered}
$$

Como $X_{\alpha_{1}} \cap X_{\alpha_{2}}$ é finito, temos que $a=a_{X_{\alpha_{1}}} \cap a_{X_{\alpha_{2}}}=a_{X_{\alpha_{1}} \cap X_{\alpha_{2}}} \in A$. Daí, $M_{\beta}=$ $N_{\beta} \cap B$, onde $B=E \cup(F \cap a) \cup(G \backslash a) \in A$, contradizendo a hipótese.

Passemos à construção da álgebra de Boole:

Teorema 7.2. Existe um corpo de conjuntos $A \subseteq \wp(\mathbb{N})$ tal que

a) todo subconjunto finito ou cofinito de $\mathbb{N}$ pertence a $A$;

b) para nenhum $X \subseteq \mathbb{N}$ infinito tem-se que $\wp(X)=\{X \cap a: a \in A\}$;

c) A tem a PCS.

Demonstração. Construiremos, por indução, uma seqüência $\left(A_{\alpha}\right)_{\alpha \in 2^{\omega}}$ de subálgebras de Boole de $\wp(\mathbb{N})$ e tomaremos $A=\bigcup_{\alpha \in 2^{\omega}} A_{\alpha}$. Fixemos primeiramente uma enumeração $\left(N_{\alpha}\right)_{\alpha \in 2^{\omega}}$ de todos os subconjuntos infinitos de $\mathbb{N}$. Fixemos também uma função $\rho: 2^{\omega} \rightarrow$ $2^{\omega} \times 2^{\omega}$ sobrejetora com a propriedade que se $\rho(\xi)=(\eta, \zeta)$, então $\eta<\xi$.

Tomamos $A_{0}$ a álgebra de todos os subconjuntos finitos e cofinitos de $\mathbb{N}$. Considere $\left(a_{n}(0, \zeta)\right)_{n \in \omega, \zeta \in 2^{\omega}}$ uma enumeração de todas as anticadeias enumeráveis de $A_{0}$. Escolhemos então $M_{0} \subseteq N_{0}$ tal que $M_{0}$ não é da forma $N_{0} \cap a \operatorname{com} a \in A_{0}$ (neste primeiro passo, basta tomar $M_{0}$ infinito com $N_{0} \backslash M_{0}$ infinito). 
Seja $\gamma<2^{\omega}$ e suponha que temos, para cada $\alpha<\gamma$, uma subálgebra $A_{\alpha}$ de $\wp(\mathbb{N})$ tal que $\left|A_{\alpha}\right|=\max \{\omega,|\alpha|\}, M_{\alpha} \subseteq N_{\alpha}$ e uma enumeração $\left(a_{n}(\alpha, \xi)\right)_{n \in \omega, \xi \in 2^{\omega}}$ de todas as anticadeias enumeráveis em $A_{\alpha}$. Suponha ainda que temos que $A_{\alpha} \subseteq A_{\beta}$ para todo $\alpha<\beta<\gamma$ e que para todos $\alpha, \beta<\gamma$, temos que $M_{\beta} \notin\left\{N_{\beta} \cap a: a \in A_{\alpha}\right\}$.

Seja $\rho(\gamma)=(\eta, \zeta)$. Pelo lema anterior, existe $\sigma \subseteq \mathbb{N}$ infinito tal que se $A_{\gamma}$ é a álgebra gerada por $\bigcup_{\alpha<\gamma} A_{\alpha}$ e $\bigcup_{n \in \sigma} a_{n}(\eta, \zeta)$, então

$$
M_{\beta} \notin\left\{N_{\beta} \cap a: a \in \bigcup_{\alpha<\gamma} A_{\alpha}\right\} .
$$

Fixemos então $\left(a_{n}(\gamma, \xi)\right)_{n \in \omega, \xi \in 2^{\omega}}$ uma enumeração de todas as anticadeias enumeráveis de $\bigcup_{\alpha<\gamma} A_{\alpha}$ e escolha $M_{\gamma} \subseteq N_{\gamma}$ que não é da forma $N_{\gamma} \cap a$ para todo $a \in A_{\gamma}$. Isto pode ser feito, uma vez que, $\left|\bigcup_{\alpha<\gamma} A_{\alpha}\right| \leq \max \{\omega,|\gamma|\}$, e daí, $\left|A_{\gamma}\right| \leq \max \{\omega,|\gamma|\}<2^{\omega}$ e $\left|\wp\left(N_{\gamma}\right)\right|=2^{\omega}$.

Considere então $A=\bigcup_{\alpha<2^{\omega}} A_{\alpha}$ e vejamos que temos as três propriedades. A propriedade a) segue da definição de $A_{0}$ e do fato que $A_{0} \subseteq A$.

Para verificar a propriedade b), tome $N \subseteq \mathbb{N}$ infinito. Temos que $N=N_{\alpha}$ para algum $\alpha<2^{\omega}$. Daí, se $M_{\alpha}=N_{\alpha} \cap a$, para algum $a \in A$, então $a \in A_{\beta}$ para algum $\beta<2^{\omega}$ e teríamos que $M_{\alpha} \in\left\{N_{\alpha} \cap a: a \in A_{\beta}\right\}$, que sabemos que não vale.

Por fim, considere $\left(a_{n}\right)_{n \in \omega} \subseteq A$ disjunta. Como a cofinalidade ${ }^{2}$ de $2^{\omega}$ é maior que $\omega$, existe $\alpha<2^{\omega}$ tal que $\left(a_{n}\right)_{n \in \omega} \subseteq A_{\alpha}$. Logo, $\left(a_{n}\right)_{n \in \omega}=\left(a_{n}(\alpha, \xi)\right)_{n \in \omega}$ para algum $\xi<2^{\omega}$. Como $\rho$ é sobrejetora, existe $\gamma \in 2^{\omega}$ tal que $\rho(\gamma)=(\alpha, \xi)$ e, portanto, $\alpha<\gamma$. Mas existe $\sigma \subseteq \mathbb{N}$ infinito tal que $\bigcup_{n \in \sigma} a_{n}=\bigcup_{n \in \sigma} a_{n}(\alpha, \xi) \in A_{\gamma} \subseteq A$. Portanto, $A$ tem a PCS.

Vejamos que sendo $K$ o espaço de Stone da álgebra construída no teorema anterior, temos que $C(K)$ não tem cópias complementadas de $c_{0}$, nem cópias de $l_{\infty}$, como queríamos. Para isso, precisamos do seguinte:

Teorema 7.3. Suponha que A é uma subálgebra de $\wp(\mathbb{N})$ que contém todos os subconjuntos finitos de $\mathbb{N}$. Então o conjunto $\left\{n^{*}: n \in \mathbb{N}\right\}$ é discreto e denso em $S(A)$, onde $n^{*}=\{X \in$ $A: n \in X\}$.

\footnotetext{
${ }^{2}$ A cofinalidade de um cardinal $\kappa$ é o menor cardinal $\lambda$ tal que existe $\left(\beta_{\alpha}\right)_{\alpha<\lambda} \subseteq \kappa$ tal que $\sup _{\alpha<\lambda} \beta_{\alpha}=$ $\kappa$.
} 
Demonstração. Primeiramente, mostremos que o conjunto é discreto em $S(A)$, isto é, que $\left\{n^{*}\right\}$ é aberto em $S(A)$. Para isso, mostremos que $\left\{n^{*}\right\}=\{n\}^{*}$. Como $\{n\} \in n^{*}$, temos que $n^{*} \in\{n\}^{*}$. Por outro lado, seja $p \in\{n\}^{*}$ e mostremos que $p=n^{*}$. Tomemos $X \in n^{*}$. Temos que $X \in A$ e $n \in X$. Mas $p \in\{n\}^{*}$ e então $\{n\} \in p$. Daí, como $p$ é filtro, $X \in p$. Assim, $n^{*} \subseteq p$. Como $n^{*}$ é ultrafiltro, segue a igualdade, para todo $p \in\{n\}^{*}$. Portanto, $\left\{n^{*}\right\}=\{n\}^{*}$, que é aberto.

Mostremos então que o conjunto é denso em $S(A)$. Tomemos um aberto $U \subseteq S(A)$, $U \neq \emptyset$ qualquer. Então, existe $a \in A$ tal que $a^{*} \subseteq U$. Seja $n \in \mathbb{N}, n \in a$. Vejamos que $n^{*} \in a^{*}$. Como $n \in a$, temos que $\{n\} \subseteq a$ e daí, $\{n\}^{*} \subseteq a^{*} \subseteq U$, pela Proposição 1.40. Assim, $\left\{n^{*}: n \in \mathbb{N}\right\}$ é denso em $S(A)$.

Teorema 7.4. Seja A uma subálgebra de $\wp(\mathbb{N})$ que possui todos os conjuntos finitos e tal que para nenhum conjunto infinito $X \subseteq \mathbb{N}$ temos que $\wp(X)=\{X \cap a: a \in A\}$. Então $D=\left\{n^{*}: n \in \mathbb{N}\right\}$ é um conjunto enumerável denso em $S(A)$ tal que para todo $D^{\prime} \subseteq D$ existem $M, N \subseteq D^{\prime}$ disjuntos tais que $\bar{M} \cap \bar{N} \neq \emptyset$.

Demonstração. Pelo teorema anterior, $D=\left\{n^{*}: n \in \mathbb{N}\right\}$ é denso em $S(A)$ e é claro que é enumerável. Seja $D^{\prime} \subseteq D$ infinito qualquer e $X \subseteq \mathbb{N}$ tal que $D^{\prime}=\left\{n^{*}: n \in X\right\}$.

Suponhamos, por absurdo, que quaisquer que sejam $M, N \subseteq D^{\prime}$ disjuntos temos $\bar{M} \cap$ $\bar{N}=\emptyset$. Mostremos que, neste caso, $\wp(X)=\{X \cap a: a \in A\}$, contradizendo a propriedade da álgebra. Tomemos $Y \subseteq X$.

Considere $M=\left\{n^{*}: n \in Y\right\}$ e $N=\left\{n^{*}: n \in X-Y\right\}$. Então existe $a \in A$ tal que $M \subseteq a^{*}$ e $N \cap a^{*}=\emptyset$. Logo, $D^{\prime} \cap a^{*}=M$ e, portanto, $X \cap a=Y$.

Vejamos, finalmente, o principal resultado desta seção:

Teorema 7.5. Existe um espaço compacto infinito $K$ tal que $C(K)$ é um espaço de Grothendieck e não tem cópias de $l_{\infty}$. Portanto, $C(K)$ não tem cópias complementadas de $c_{0}$ nem de $l_{\infty}$.

Demonstração. Considere $A$ uma álgebra como no Teorema 7.2 e seja $K$ seu espaço de Stone. Como $A$ tem a PCS, pelo Teorema 3.47, temos que $C(K)$ é Grothendieck e, portanto, pelo Teorema 3.44 , não tem cópias complementadas de $c_{0}$. 
Vejamos que $C(K)$ não tem cópias de $l_{\infty}$. Suponhamos por absurdo que existe $Y \subseteq$ $\mathcal{C}(K)$ e $T: l_{\infty} \longrightarrow Y$ um isomorfismo. Daí, $T^{-1}$ é limitada e portanto, $T$ não diminui as normas arbitrariamente, isto é, existe $\delta>0$ tal que $\left\|T\left(e_{n}\right)\right\|>\delta$ para todo $n \in \omega$, onde $\left(e_{n}\right)_{n \in \omega}$ é a base canônica de $l_{\infty}$. Como $\left\{k^{*}: k \in \mathbb{N}\right\}$ é denso em $K$, temos que $\sup _{t \in K}\left|T\left(e_{n}\right)(t)\right|=\sup _{k \in \mathbb{N}}\left|T\left(e_{n}\right)\left(k^{*}\right)\right|$. Assim, para cada $n \in \mathbb{N}$, existe $m_{n} \in \mathbb{N}$ tal que $\left|T\left(e_{n}\right)\left(m_{n}{ }^{*}\right)\right|>\delta$. Consideremos então, para cada $k \in \mathbb{N}$ o funcional $\phi_{k}$ em $l_{\infty}$ definido por $\phi_{k}=T^{*}\left(\delta_{m_{k}}\right) \in l_{\infty}^{*}$, onde $\delta_{x}$ é o funcional de evaluação em $x$ de norma 1 .

Para cada $k \in \mathbb{N}$ e cada $n \in \mathbb{N}$, defina $a_{n}^{k}=\phi_{k}\left(e_{n}\right)$. Vejamos que tais seqüências satisfazem as hipóteses do Lema de Rosenthal (3.36): para $k \in \mathbb{N}$ e $m \in \mathbb{N}$ fixos, temos:

$$
\begin{aligned}
\sum_{n=1}^{m}\left|a_{n}^{k}\right| & =\sum_{n=1}^{m}\left|\phi_{k}\left(e_{n}\right)\right|=\sum_{n=1}^{m}(-1)^{\sigma_{n}} \phi_{k}\left(e_{n}\right) \\
& =\sum_{n=1}^{m} \phi_{k}\left((-1)^{\sigma_{n}} e_{n}\right)=\phi_{k}\left(\sum_{n=1}^{m}(-1)^{\sigma_{n}} e_{n}\right) \\
& =T\left(\sum_{n=1}^{m}(-1)^{\sigma_{n}} e_{n}\right)\left(m_{k}{ }^{*}\right) \leq\left\|T\left(\sum_{n=1}^{m}(-1)^{\sigma_{n}} e_{n}\right)\right\| \\
& \leq\|T\| \cdot\left\|\sum_{n=1}^{m}(-1)^{\sigma_{n}} e_{n}\right\|=\|T\| .
\end{aligned}
$$

Pelo Lema de Rosenthal (3.36) e pelo Lema 3.35, temos que existe $N \subseteq \mathbb{N}$ infinito tal que para todo $k \in N$, temos

$$
\sum_{n \in N \backslash\{k\}}\left|\phi_{k}\left(e_{n}\right)\right|<\frac{\delta}{3}
$$

e para todo $M \subseteq N$ temos

$$
\phi_{k}\left(\chi_{M}\right)=\sum_{n \in M} \phi_{k}\left(e_{n}\right)
$$

Vejamos que para todo $M \subseteq N$ temos que existem $F_{1}, F_{2} \subseteq K$ fechados disjuntos tais que $\left\{m_{k}{ }^{*}: k \in M\right\} \subseteq F_{1}$ e $\left\{m_{k}{ }^{*}: k \in N \backslash M\right\} \subseteq F_{2}$, contradizendo o teorema anterior.

Para cada $M \subseteq N$, temos que

$$
\left|T\left(\chi_{M}\right)\left(m_{k}^{*}\right)\right|=\left|\phi_{k}\left(\chi_{M}\right)\right|=\left|\sum_{n \in M} \phi_{k}\left(e_{n}\right)\right|=\left|\sum_{n \in M} T\left(e_{n}\right)\left(m_{k}^{*}\right)\right| .
$$

Daí, se $k \in M$, temos

$$
\left|\sum_{n \in M} T\left(e_{n}\right)\left(m_{k}^{*}\right)\right|=\left|T\left(e_{k}\right)\left(m_{k}^{*}\right)+\sum_{n \in M \backslash\{k\}} T\left(e_{n}\right)\left(m_{k}^{*}\right)\right|>\frac{2 \delta}{3}
$$

e se $k \notin M$ temos

$$
\left|\sum_{n \in M} T\left(e_{n}\right)\left(m_{k}^{*}\right)\right|=\left|\sum_{n \in M \backslash\{k\}} T\left(e_{n}\right)\left(m_{k}^{*}\right)\right|<\frac{\delta}{3} .
$$


Tomemos então $F_{1}=T\left(\chi_{M}\right)^{-1}\left(\left[-\frac{\delta}{3}, \frac{\delta}{3}\right]\right)$ e $F_{2}=T\left(\chi_{M}\right)^{-1}\left(\left[-\|T\|,-\frac{2 \delta}{3}\right] \cup\left[\frac{2 \delta}{3},\|T\|\right]\right)$, que são fechados disjuntos, pois são imagens inversas de fechados disjuntos por uma função contínua.

\subsection{A densidade de espaços de Grothendieck $C(K)$}

Como já dissemos, pretendemos, nesta última seção, apresentar a demonstração da independência da seguinte afirmação:

"Existe um espaço booleano $K$ tal que $C(K)$ é Grothendieck e $d(C(K))<2^{\omega}$."

Sabemos, dos Teoremas 1.55 e 3.4, que o peso de um espaço booleano $K$ coincide com a cardinalidade da álgebra $C l o p(K)$ e com a densidade de $C(K)$. Assim, provar isto é o mesmo que provar a independência da existência de um espaço booleano $K$ de peso menor que $2^{\omega}$ tal que $C(K)$ é Grothendieck. Veremos primeiramente a demonstração da consistência da negação desta afirmação, que já é um resultado conhecido:

Teorema 7.6. (MA) Se $K$ é um espaço booleano infinito com peso ${ }^{3}$ menor que $2^{\omega}$, então $C(K)$ possui uma cópia complementada de $c_{0} e$, portanto, não é Grothendieck.

Demonstração. O resultado segue diretamente da Proposição 1.38 e do Teorema 3.30 .

Meu segundo objetivo aqui é mostrar que alguma hipótese adicional (MA, por exemplo) é necessária para mostrar isto, ou seja, que a existência de um espaço booleano $K$ com peso menor que $2^{\omega}$ tal que $C(K)$ é um espaço de Grothendieck, não leva a nenhuma contradição e, portanto, juntamente com o resultado acima enunciado, é independente.

O modelo em que mostrarei o resultado é o modelo obtido usando um produto de $\kappa \geq \omega_{2}$ forcing's de Sacks. Vimos que, neste modelo $\omega_{1}<2^{\omega}$. A idéia de mostrar a existência de um espaço topológico compacto $K$ com peso menor que $2^{\omega}$ tal que $C(K)$ é Grothendieck foi motivada por um resultado em [JK], onde mostra-se que, naquele modelo, um certo espaço booleano $K$ de peso $\omega_{1}<2^{\omega}$ não tem seqüências convergentes não triviais.

\footnotetext{
${ }^{3}$ Convém lembrar que na demonstração da Proposição 1.38, usamos apenas o fato que $K$ tem caráter menor que $2^{\omega}$, uma hipótese mais fraca. Aqui acontece o mesmo: a hipótese que o peso do espaço é menor que $2^{\omega}$ pode ser substituída pela hipótese de que o caráter de do espaço é menor que $2^{\omega}$. Veja [Eng] para a definição de caráter de um espaço topológico.
} 
Tal resultado pode ser interpretado como um resultado sobre medidas concentradas nos pontos de uma seqüência. Por outro lado, pelo Teorema de Representação de Riesz (3.13), podemos identificar os elementos de $C(K)^{*}$ com medidas de Radon sobre $K$. Assim, a propriedade de Grothendieck diz respeito a propriedades destes objetos. Como medidas concentradas em pontos são casos particulares de medidas de Radon, generalizando e modificando as técnicas usadas em [JK], é possível mostrar que $C(K)$ é um espaço de Grothendieck onde $K$ é o espaço booleano considerado no artigo e que $K$ tem peso $\omega_{1}<2^{\omega}$ e, portanto, $C(K)$ tem densidade $\omega_{1}<2^{\omega}$. O espaço $K$ com tais propriedades será o espaço de Stone de uma álgebra de Boole $A$. Como $K$ tem peso menor que $2^{\omega}$, sabemos que $|A|<2^{\omega}$.

Notemos que se $A$ é uma álgebra de Boole infinita com PCS, então $|A|=2^{\omega}$ : basta tomar $\left(a_{n}\right)_{n \in \mathbb{N}} \subseteq A$ uma anticadeia e considerar $\left(K_{\alpha}\right)_{\alpha<2^{\omega}} \subseteq \wp(\mathbb{N})$ quase disjunta. Daí, pela PCS, existe, para cada $\alpha<2^{\omega}, M_{\alpha} \subseteq K_{\alpha}$ infinito tal que $\sum_{n \in M_{\alpha}} a_{n} \in A$. Como $\left(K_{\alpha}\right)_{\alpha<2^{\omega}}$ é quase disjunta, temos que se $\alpha_{1} \neq \alpha_{2}$, então $\sum_{n \in M_{\alpha_{1}}} a_{n} \neq \sum_{n \in M_{\alpha_{2}}} a_{n}$. Como a álgebra que construímos possui menos que $2^{\omega}$ elementos, segue que ela não possui PCS em $M[G]$.

Existem, na literatura, diversas outras propriedades para álgebras de Boole $A$ que implicam que $C(S(A))$ tem a propriedade de Grothendieck. Porém, muitas delas também implicam que $|A| \geq 2^{\omega}$. Por exemplo, a propriedade de interpolação subseqüencial ${ }^{4}$ : para toda anticadeia $\left(a_{n}\right)_{n \in \mathbb{N}} \subseteq A$ e para todo $M \subseteq \mathbb{N}$ infinito, existe $N \subseteq M$ infinito e $a \in A$ tal que $a_{n} \leq a$ para todo $n \in N$ e $a_{n} \leq-a$ para todo $n \in M \backslash N$. Como a álgebra que construiremos no último resultado tem cardinalidade $\omega_{1}<2^{\omega}$ em $M[G]$, segue que ela não possui nenhuma destas propriedades. O que tais propriedades têm em comum, é que elas exigem que a álgebra possua muitas uniões de anticadeias e isto faz com que sua cardinalidade seja grande.

Por outro lado, para uma álgebra de Boole $A$ ter a propriedade de Grothendieck, ela precisa possuir, para cada elemento de $C(S(A))^{* *}$ que separa uma seqüência de $C(S(A))^{*}$ na topologia fraca, um elemento que separe esta mesma seqüência na topologia fraca*. Assim, parece-nos que uma álgebra de Boole com a propriedade de Grothendieck precisa

\footnotetext{
${ }^{4}$ Algumas outras propriedades deste tipo ((f)-propriedade, completude subseqüencial fraca, propriedade de interpolação subseqüencial fraca, etc) podem ser encontradas em [Ai] e implicam que a cardinalidade da álgebra é maior que $2^{\omega}$.
} 
ser "grande", num certo sentido.

A álgebra de Boole que consideraremos é a álgebra $\wp(\mathbb{N}) \cap M$, onde $M$ é o modelo inicial do forcing. Para mostrar que se $K$ é o espaço de Stone dela no modelo $M[G]$ obtido com o forcing de Sacks, então $C(K)$ é um espaço de Grothendieck, precisamos de diversos resultados combinatórios:

Lema 7.7. Sejam $m, A, N \in \mathbb{N}$ e $X \subseteq \mathbb{N}$ infinito. Dada uma família $\left\{G_{i}: i<N\right\}$ com $\left|G_{i}\right| \geq A+m$ e dados $X_{k, i} \subseteq X$ com $i<N$ e $k \in G_{i}$ tais que

$$
X \subseteq \bigcup_{k \in F} X_{k, i}
$$

para todo $F \subseteq G_{i}$ com $|F| \geq m$ e todo $i<N$, existem $H_{i} \subseteq G_{i}$ com $\left|H_{i}\right|=A$ tais que

$$
\bigcap\left\{X_{k, i}: k \in H_{i}, i<N\right\} \text { é infinito. }
$$

Demonstração. Provemos o resultado por indução em $N$. Seja $X \subseteq \mathbb{N}$ infinito e $A, m \in \mathbb{N}$. Para $N=0$, o resultado vale por vacuidade.

Suponhamos que o resultado vale para $N$ e provemos para $N+1$. Suponha então $\left\{G_{i}: i<N+1\right\}$ uma família de conjuntos com $\left|G_{i}\right| \geq A+m$ e $X_{k, i}$, para $0 \leq i<N+1$ e $k \in G_{i}$ como na hipótese. Temos que existem, para cada $0 \leq i<N, H_{i} \subseteq G_{i}$ com $\left|H_{i}\right|=A$ tais que

$$
Y:=\bigcap_{k \in H_{i}, 0 \leq i<N} X_{k_{i}} \text { é infinito. }
$$

Vamos construir, para $0 \leq l<A, G^{(l)}$ e $k_{l} \in G^{(l)}$ tais que $G^{(0)} \subseteq G_{N}, G^{(l)} \subseteq G_{N} \backslash\left\{k_{i}\right.$ : $0 \leq i<l\},\left|G^{(l)}\right|=m$ e $Y \cap X_{k_{0}, N} \cap \cdots \cap X_{k_{l}, N}$ é infinito.

Daí, tomando $H_{N}=\left\{k_{j}: 0 \leq j<A\right\}$, temos que $\left|H_{N}\right|=A$ e

$$
\bigcap_{k \in H_{i}, i<N+1} X_{k, i}=Y \cap\left(\bigcap_{k \in H_{N}} X_{k, N}\right) \text { é infinito, }
$$

e temos a tese.

Para a construção, considere $G^{(0)} \subseteq G_{N}$ com $\left|G^{(0)}\right|=m$. Pela hipótese, $Y \subseteq X \subseteq$ $\bigcup_{k \in G^{(0)}} X_{k, N}$. Como $Y$ é infinito, existe $k_{0} \in G^{(0)}$ tal que $Y \cap X_{k_{0}, N}$ é infinito.

Se $A=1$, temos o resultado. Senão, suponhamos $0<l<A$ e que temos $k_{0}, \ldots, k_{l-1} \in$ $G_{N}$, tais que $X_{k_{0}, N} \cap \cdots \cap X_{k_{l-1}, N} \cap Y$ é infinito. Daí, como $\left|G_{N} \backslash\left\{k_{i}: 0 \leq i<l\right\}\right|=$ 
$\left|G_{N}\right|-l \geq A+m-l \geq m$, existe $G^{(l)} \subseteq G_{N} \backslash\left\{k_{i}: 0 \leq i<l\right\}$ com $\left|G^{(l)}\right| \geq m$. Então, temos que

$$
Y \cap\left(X_{k_{0}, N} \cap \cdots \cap X_{k_{l-1}, N}\right) \subseteq X \subseteq \bigcup_{k \in G^{(l)}} X_{k, N} .
$$

Portanto, existe $k_{l} \in G^{(l)}$ tal que $X_{k_{0}, N} \cap \cdots \cap X_{k_{l}, N}$ é infinito, concluindo assim a demonstração.

Lema 7.8. Dados $G \subseteq \mathbb{N}$ finito, $K \in \mathbb{N}$ e para cada $k<K$, $\left(m_{n}^{k}\right)_{n \in G}$ uma seqüência de reais positivos e $M$ um real positivo tais que para todo $k<K, \sum_{n \in G} m_{n}^{k} \leq M$ e dados $\eta$ um real positivo e $m \in \mathbb{N}$ tais que $\eta \cdot m>M$, existe $G^{\prime} \subseteq G$, com $\left|G^{\prime}\right|=|G|-K \cdot m$ e tal que para todo $k<K$, todo $n \in G^{\prime}$ tem-se $m_{n}^{k}<\eta$.

Demonstra̧̧ão. Se $|G| \leq K \cdot m$, o resultado segue tomando $G^{\prime}=\emptyset$. Suponhamos então $|G|>K \cdot m$.

Se existissem $n_{1}, \ldots, n_{m} \in G$ tais que $m_{n_{i}}^{0} \geq \eta$ para todo $1 \leq i \leq m$, então

$$
\sum_{1 \leq i \leq m} m_{n_{i}}^{0} \geq m \cdot \eta>M
$$

contradizendo a hipótese. Logo, existe $G_{0} \subseteq G$ com $\left|G_{0}\right|=|G|-m$ tal que para todo $n \in G_{0}$ tem-se que para todo $n \in G_{0}, m_{n}^{0}<\eta$.

Construímos, por indução, para cada $0<k<K, G_{k} \subseteq G_{k-1} \operatorname{com}\left|G_{k}\right|=\left|G_{k-1}\right|-m$ e tal que para todo $n \in G_{k}, m_{n}^{k}<\eta$. Isto pode ser feito, pois se existissem $n_{1}, \ldots, n_{m} \in G_{k-1}$ tais que $m_{n_{i}}^{k} \geq \eta$ para todo $1 \leq i \leq m$, então

$$
\sum_{1 \leq i \leq m} m_{n_{i}}^{k} \geq m \cdot \eta>M
$$

Tomemos $G^{\prime}=G_{K-1}$ e temos que

$$
\left|G^{\prime}\right|=\left|G_{K-1}\right|=\left|G_{K-2}\right|-m=\cdots=\left|G_{0}\right|-(K-1) \cdot m=|G|-K \cdot m
$$

e $\forall k<K \quad \forall n \in G^{\prime} \quad m_{n}^{k}<\eta$.

Lema 7.9. Seja $N \in \mathbb{N}$, sejam, para cada $i<N, G_{i} \subseteq \mathbb{N}$ finito, $K \in \mathbb{N}$ e para cada $k<K$ e cada $i, j<N,\left(m_{n, i}^{k}(j)\right)_{n \in G_{i}}$ uma seqüência de reais positivos e seja $M$ um real positivo tais que para todo $k<K$, todos $i, j<N, \sum_{n \in G_{i}} m_{n, i}^{k}(j) \leq M$. Dados $\eta$ 
um real positivo e $m \in \mathbb{N}$ tais que $\eta \cdot m>M$, existe, para cada $i<N, G_{i}^{\prime} \subseteq G_{i}$, com $\left|G_{i}^{\prime}\right|=\left|G_{i}\right|-N \cdot K \cdot m$ e tal que para todo $k<K$, todos $i, j<N$ e todo $n \in G_{j}^{\prime}$ tem-se $m_{n, i}^{k}(j)<\eta$.

Demonstração. Fixemos $j<N$. Pelo lema anterior, existe $G_{j}^{0} \subseteq G_{j}$ com $\left|G_{j}^{0}\right|=\left|G_{j}\right|-$ $K \cdot m$ e tal que para todo $k<K$ e todo $n \in G_{j}^{0}$ tem-se $m_{n, 0}^{k}(j)<\eta$.

Construímos, então, por indução, para cada $0<i<N, G_{j}^{i} \operatorname{com} G_{j}^{i+1} \subseteq G_{j}^{i},\left|G_{j}^{i+1}\right|=$ $\left|G_{j}^{i}\right|-K \cdot m$ e tais que para todo $k<K$ e todo $n \in G_{j}^{i}$ tem-se $m_{n, i}^{k}(j)<\eta$.

Tomemos então $G_{j}^{\prime}=G_{j}^{N-1}$. Temos que

$\left|G_{j}^{\prime}\right|=\left|G_{j}^{N-1}\right|=\left|G_{j}^{N-2}\right|-K \cdot m=\cdots=\left|G_{j}^{0}\right|-(N-1) \cdot K \cdot m=\left|G_{j}\right|-N \cdot K \cdot m$

e para cada $i<N$, cada $n \in G_{j}$ e cada $k<K$, tem-se $m_{n, i}^{k}(j)<\eta$.

Lema 7.10. Sejam $N \in \mathbb{N}$ e para cada $i<N, G_{i} \subseteq \mathbb{N}$ finito. Sëa ainda, para cada $k \in \mathbb{N}$ e cada $i, j<N,\left(m_{n, i}^{k}(j)\right)_{n \in G_{i}}$ uma seqüência de reais positivos e seja $M$ um real positivo tais que

$$
\forall k \in \mathbb{N} \quad \forall i, j<N \quad \sum_{n \in G_{i}} m_{n, i}^{k}(j) \leq M .
$$

Dados $\eta$ um real positivo e $m \in \mathbb{N}$ tais que $\eta \cdot m>M$, e dado $C \in \mathbb{N}$, existe $B \in \mathbb{N}$ tais que se para todo $i<N\left|G_{i}\right| \geq B$, então existe, para cada $i<N, G_{i}^{*} \subseteq G_{i}$, com $\left|G_{i}^{*}\right|=C$ e tal que

$$
\forall i, j<N \quad i \neq j \quad \forall k \in G_{i}^{*} \quad \forall n \in G_{j}^{*} \quad m_{n, i}^{k}(j)<\eta
$$

Demonstração. Provemos por indução em $N$. Se $N=1$, não há nada a ser feito.

Suponhamos agora que o resultado vale para $N$ e provemos para $N+1$. Primeiramente, seja $m \in \mathbb{N}$ tal que $\eta \cdot m>M$. Dado $C \in \mathbb{N}$, seja $C^{\prime}=C+N \cdot C \cdot m$ e temos que existe $B^{\prime} \in \mathbb{N}$ satisfazendo o resultado para $N$. Considere $B=C+N^{2} \cdot C^{\prime} \cdot m$.

Dados $G_{0}, \ldots, G_{N}$ e $m_{n, i}^{k}(j)$ satisfazendo $(*)$, temos que, para $0 \leq i<N$, existem $G_{i}^{* *} \subseteq G_{i} \operatorname{com}\left|G_{i}^{* *}\right|=C^{\prime}$ satisfazendo ${ }^{* *}$. Considere $K=\bigcup_{i<N} G_{i}^{* *}$. Temos que $|K| \leq N \cdot C^{\prime}$. Pelo Lema 7.9, existe $G_{N}^{*} \subseteq G_{N}$ com

$$
\left|G_{N}^{*}\right|=\left|G_{N}\right|-N \cdot|K| \cdot m=B-N \cdot|K| \cdot m \geq C+N^{2} \cdot C^{\prime} \cdot m-N^{2} \cdot C^{\prime} \cdot m=C
$$

e para todo $k \in K$, todo $n \in G_{N}^{*}$ e todo $i<N, m_{n, i}^{k}(N)<\eta$. 
Podemos supor, sem perda de generalidade, que $\left|G_{N}^{*}\right|=C$.

Daí, pelo Lema 7.9, existe, para cada $i<N, G_{i}^{*} \subseteq G_{i}^{* *}$ com

$$
\left|G_{i}^{*}\right|=\left|G_{i}^{* *}\right|-N \cdot\left|G_{N}^{*}\right| \cdot m=C+N \cdot C \cdot m-N \cdot C \cdot m=C
$$

e tais que para todo $n \in G_{i}^{*}$ e todo $k \in G_{N}^{*}, m_{n, N}^{k}(i)<\eta$ e temos o resultado.

Temos assim, a seguinte proposição, que fornece o passo indutivo da construção por indução que faremos na demonstração do resultado principal desta seção. Na realidade, tal proposição permite-nos fazer a demonstração do resultado principal de uma maneira análoga à feita no Teorema 3.47. Vejamos, primeiramente, sua demonstração:

Proposição 7.11. Seja $N \in \mathbb{N}, N \geq 1$. Suponhamos $s_{0}, \ldots, s_{N} \in \mathbb{S}^{\kappa}, \varepsilon, M>0$ e $E \subseteq \mathbb{N}$ finito e suponha que $\left(\dot{\mu}_{k}\right)_{k \in \mathbb{N}}$ uma seqüência de nomes para medidas finitamente aditivas sobre a álgebra de Boole $\wp(\mathbb{N})$, e $\left(\dot{A}_{k}\right)_{k \in \mathbb{N}}$ uma seqüências de nomes para subconjuntos de $\mathbb{N}$ e $\dot{X}_{N}$ é um nome para um subconjunto de $\mathbb{N}$ tais que

$$
s_{N} \Vdash\left\{\begin{array}{l}
\forall k \in \mathbb{N} \quad\left\|\dot{\mu}_{k}\right\|<\check{M}, \\
\forall k, k^{\prime} \in \mathbb{N} \quad k \neq k^{\prime} \quad \dot{A}_{k} \cap \dot{A}_{k^{\prime}}=\emptyset, \\
\dot{X}_{N} \subseteq\left\{k \in \mathbb{N}:\left|\dot{\mu}_{k}\left(\dot{A}_{k}\right)\right| \geq \varepsilon\right\}, \dot{X}_{N} \text { é infinito, } \\
\check{E} \cap \dot{X}_{N}=\emptyset .
\end{array}\right.
$$

Seja ainda, $F_{N}=\left\{\alpha_{i}^{k}: i, k<N\right\}$, onde $\operatorname{supp}\left(s_{i}\right)=\left\{\alpha_{i}^{k}: k \in \mathbb{N}\right\}$. Então, dado $\delta>0$, existem $s_{N+1} \in \mathbb{S}^{\kappa}$, com $s_{N+1} \leq_{F_{N}, N} s_{N}, a_{N+1} \subseteq \mathbb{N}$ e $E_{N+1} \subseteq \mathbb{N}$ com $\left|E_{N+1}\right|=2^{N\left|F_{N}\right|} e$ uma seqüência de nomes $\left(\dot{A}_{k}^{\prime}\right)_{k \in \mathbb{N}}$ para subconjuntos dos naturais e $\dot{X}_{N+1}$ um nome para um subconjunto dos naturais tais que

$$
s_{N+1} \Vdash\left\{\begin{array}{l}
\forall k, k^{\prime} \in \mathbb{N} \quad k \neq k^{\prime} \quad \dot{A}_{k}^{\prime} \cap \dot{A}_{k^{\prime}}^{\prime}=\emptyset, \\
\dot{X}_{N+1} \subseteq\left\{k \in \dot{X}_{N}:\left|\dot{\mu}_{k}\left(\dot{A}_{k}^{\prime}\right)\right| \geq \varepsilon-\delta\right\}, \dot{X}_{N+1} \text { é infinito, } \\
\check{E}_{N+1} \subseteq \dot{X}_{N} \backslash \dot{X}_{N+1}, \\
\forall k \in \dot{X}_{N+1} \quad\left|\dot{\mu}_{k}\left(\check{a}_{N+1}\right)\right| \leq \delta, \\
\forall k \in \check{E} \quad\left|\dot{\mu}_{k}\left(\check{a}_{N+1}\right)\right| \leq \delta, \\
\exists k \in \check{E}_{N+1} \quad\left|\dot{\mu}_{k}\left(\check{a}_{N+1}\right)\right|>\varepsilon-\delta .
\end{array}\right.
$$

Demonstração. Primeiramente, seja $K=2^{N\left|F_{N}\right|}$ e seja $\eta=\frac{\delta}{K}$. Fixemos então $m \in \mathbb{N}$ tal que $m \eta>M$. Seja $C \in \mathbb{N}$ suficientemente grande (aplicaremos os Lemas 7.7, 7.8, 7.9 e 7.10 
diversas vezes e, assim, diminuiremos este $C$ muitas vezes. Precisamos ele suficientemente grande para, ao aplicarmos tais lemas todas as vezes necessárias, tenhamos ainda um conjunto não vazio) e seja $B \in \mathbb{N}$ como na tese do Lema 7.10, para $C$.

Nosso $a_{N+1}$ será a união de alguns $\dot{A}_{k}$, para alguns $k \in \dot{X}_{N}$. Queremos então achar tais $k$ 's. Para começar, queremos decidir muitos possíveis candidatos para $k$ 's, para depois tirar sucessivamente aqueles que não nos servem. Para isso, precisamos começar decidindo uma parte grande finita (de tamanho $B$ ) de $\dot{X}_{N+1}$. Para isso, considere $L=l\left(F_{N}, N, s_{N}\right)$ e seja

$$
D=\left\{p \in \mathbb{S}^{\kappa}: \exists G \subseteq \mathbb{N},|G|=B, p \Vdash \check{G} \subseteq \dot{X}_{N}\right\} .
$$

É fácil ver que $D$ é denso abaixo de $s_{N}$, pois $s_{N} \Vdash \dot{X}_{N}$ é infinito. $D$ é aberto, pois se $s \in D$ e $t \leq s$, então o mesmo $G$ que testemunha que $s \in D$ também testemunha que $t \in D$. Assim, pelo Lema 4.68, existe $s_{N}^{\prime} \in \mathbb{S}^{\kappa}$ tal que $s_{N}^{\prime} \leq_{F_{N}, N} s_{N}$ e

$$
\forall \sigma \in L \quad s_{N}^{\prime} \mid \sigma \in D
$$

Daí, para cada $\sigma \in L$, existe $G_{\sigma} \subseteq \mathbb{N}$ com $\left|G_{\sigma}\right|=B$ tal que

$$
s_{N}^{\prime} \mid \sigma \Vdash \check{G}_{\sigma} \subseteq \dot{X}_{N}
$$

Precisamos então decidir quem são os $\dot{A}_{k}$, para $k \in G_{\sigma}$, para todo $\sigma \in L$, para então trabalharmos com eles. Seja então, para cada $\sigma \in L$,

$$
D_{\sigma}=\left\{p \in \mathbb{S}^{\kappa}: \forall k \in G_{\sigma} \exists A_{k} \subseteq \mathbb{N} p \Vdash \check{A}_{k}=\dot{A}_{k}\right\}
$$

Novamente, é fácil ver que cada $D_{\sigma}$ é denso abaixo de $s_{N}^{\prime}$, pois $s_{N}^{\prime} s_{N} \Vdash \dot{A}_{k} \in \wp(\mathbb{N}) \cap M$ para todo $k \in \mathbb{N}$. Cada $D_{\sigma}$ é aberto, pois se $s \in D_{\sigma}$ e $t \leq s$, então os mesmos $A_{k}$ 's que testemunham que $s \in D_{\sigma}$ também testemunha que $t \in D_{\sigma}$. Assim, temos que cada $D_{\sigma}$ é aberto denso abaixo de $s_{N}^{\prime}$ e intersecção finita de abertos densos é um aberto denso. Daí, pelo Lema 4.68, existe $s_{N}^{\prime \prime} \in \mathbb{S}^{\kappa} \operatorname{com} s_{N}^{\prime \prime} \leq_{F_{N}, N} s_{N}^{\prime}$ tal que para todo $\sigma \in L$, tem-se que $s_{N}^{\prime \prime} \mid \sigma \in \bigcap_{\sigma^{\prime} \in L} D_{\sigma^{\prime}}$. Portanto, para cada $\sigma \in L$, e cada $k \in G_{\sigma}$, seja $A_{k}(\sigma) \subseteq \mathbb{N}$ tal que

$$
s_{N}^{\prime \prime} \mid \sigma \Vdash \check{A}_{k}(\sigma)=\dot{A}_{k} .
$$

Como queremos algumas propriedades sobre as medidas de $a_{N+1}((4),(5),(6))$ e dos nomes $\dot{A}_{n}^{\prime}((2))$, precisamos trabalhar com as medidas de $A_{n}(\sigma)$ e, portanto, aproximálas. Achamos, usando o Lema 4.68, $t_{N} \in \mathbb{S}^{\kappa} \operatorname{com} t_{N} \leq_{F_{N}, N} s_{N}^{\prime \prime}$ e, para cada $\sigma, \sigma^{\prime} \in L$, 
cada $k \in G_{\sigma}$ e cada $n \in G_{\sigma^{\prime}}$, achamos $m_{n, \sigma}^{k}\left(\sigma^{\prime}\right) \in \mathbb{R}$ tais que $\forall \sigma \neq \sigma^{\prime} \in L, \forall k \in G_{\sigma}$,

$$
\sum_{n \in G_{\sigma^{\prime}}} m_{n, \sigma}^{k}\left(\sigma^{\prime}\right)<M \quad \text { e } \quad \forall n \in G_{\sigma^{\prime}} \quad t_{N}|\sigma \Vdash| \dot{\mu}_{k} \mid\left(\check{A}_{n}\left(\sigma^{\prime}\right)\right) \leq \check{m}_{n, \sigma}^{k}\left(\sigma^{\prime}\right) .
$$

Para isso, considere $\left\{\sigma_{i}: i<K\right\}$ uma enumeração de $L$ e construímos, por indução, para cada $i<K, t_{N}^{i} \in \mathbb{S}^{\kappa}$, onde $t_{N}^{0} \leq_{F_{N}, N} s_{N}^{\prime \prime}$ e com $t_{N}^{i+1} \leq_{F_{N}, N} t_{N}^{i}$ e para $j<K, j \neq i$, $k \in G_{\sigma_{i}}$ e $n \in G_{\sigma_{j}}, m_{n, \sigma_{i}}^{k}\left(\sigma_{j}\right) \in \mathbb{R}$ tais que $\sum_{n \in G_{\sigma_{j}}} m_{n, \sigma_{i}}^{k}\left(\sigma_{j}\right)<M$ e

$$
t_{N}^{i}\left|\sigma_{i} \Vdash\right| \dot{\mu}_{k} \mid\left(\check{A}_{n}\left(\sigma_{j}\right)\right)<\check{m}_{n, \sigma_{i}}^{k}\left(\sigma_{j}\right) .
$$

Para tanto, fixemos $0 \leq i<K-1$ e suponhamos que temos $t_{N}^{i}$ como acima. Daí, como $s_{N} \Vdash \forall k \in \mathbb{N},\left\|\dot{\mu}_{k}\right\|<M$, existe $\left(t_{N}^{i+1}\right)^{\prime} \leq_{F_{N}, N} t_{N}^{i}$ e $\theta_{j}^{i+1} \in \mathbb{R}$ tal que

$$
\left(t_{N}^{i+1}\right)^{\prime} \Vdash \check{\theta}_{j}^{i+1} \leq M-\sum_{n \in G_{\sigma_{j}}}\left|\dot{\mu}_{k}\right|\left(\check{A}_{n}\left(\sigma_{j}\right)\right) .
$$

Então, existem $m_{n, \sigma_{i+1}}^{k}\left(\sigma_{j}\right) \in \mathbb{R}$ e $t_{N}^{i+1} \leq_{F_{N}, N}\left(t_{N}^{i+1}\right)^{\prime}$ tais que

$$
t_{N}^{i+1} \Vdash 0 \leq \check{m}_{n, \sigma_{i+1}}^{k}\left(\sigma_{j}\right)-\left|\dot{\mu}_{k}\right|\left(\check{A}_{n}\left(\sigma_{j}\right)\right)<\frac{\check{\theta}_{j}^{i+1}}{\left|G_{\sigma_{j}}\right|} .
$$

Portanto, $\sum_{n \in G_{\sigma_{j}}} m_{n, \sigma_{i+1}}^{k}\left(\sigma_{j}\right)<M$ para todo $j<K, j \neq i+1 \mathrm{e}$

$$
t_{N}^{i+1} \Vdash\left|\dot{\mu}_{k}\right|\left(\check{A}_{n}\left(\sigma_{j}\right)\right) \leq \check{m}_{n, \sigma_{i+1}}^{k}\left(\sigma_{j}\right) .
$$

Tomemos $t_{N}=t_{N}^{K-1}$ e temos $(*)$.

Para obtermos (2), (4), (6), queremos tais medidas pequenas. Pelo Lema 7.10, achamos $G_{\sigma}^{*} \subseteq G_{\sigma}$ com $\left|G_{\sigma}^{*}\right|=C$ tais que

$$
\forall i, j<K \quad i \neq j, \quad \forall k \in G_{\sigma_{i}}^{*} \quad \forall n \in G_{\sigma_{j}}^{*} \quad m_{n, \sigma_{i}}^{k}\left(\sigma_{j}\right)<\eta .
$$

Queremos também controlar as medidas com índices em $E$, para obter (5). Para isso, achamos analogamente usando o Lema 4.68 e o fato que $s_{N} \Vdash \forall k \in \mathbb{N},\left\|\dot{\mu}_{k}\right\|<M$, $t_{N}^{\prime} \leq_{F_{N}, N} t_{N}$ e para cada $\sigma, \sigma^{\prime} \in L$, cada $k \in E$ e cada $n \in G_{\sigma}^{*}, m_{n, \sigma}^{k}\left(\sigma^{\prime}\right) \in \mathbb{R}$ tais que

$$
t_{N}^{\prime}|\sigma \Vdash| \dot{\mu}_{k}\left(\check{A}_{n}\left(\sigma^{\prime}\right)\right) \mid \leq m_{n, \sigma}^{k}\left(\sigma^{\prime}\right),
$$

e para todos $\sigma, \sigma^{\prime} \in L$ e todo $k \in E$

$$
\sum_{n \in G_{\sigma^{\prime}}^{*}} m_{n, \sigma}^{k}\left(\sigma^{\prime}\right)<M
$$


Como $C$ é suficientemente grande, pelo Lema 7.9, podemos supor, sem perda de generalidade, que

$$
\forall \sigma, \sigma^{\prime} \in L \quad \forall k \in E \quad \forall n \in G_{\sigma}^{*} \quad m_{n, \sigma}^{k}\left(\sigma^{\prime}\right)<\eta
$$

Para obter $\dot{X}_{N+1}$ satisfazendo (2), considere, para cada $\sigma \in L$ e $k \in G_{\sigma}^{*}$, um nome $\dot{X}_{k, \sigma}$ tal que

$$
t_{N}^{\prime} \Vdash \dot{X}_{k, \sigma}=\left\{l \in X_{N}:\left|\dot{\mu}_{l}\right|\left(\check{A}_{k}(\sigma)\right)<\eta\right\} .
$$

Lembramos que $L=\left\{\sigma_{i}: i<K\right\}$. Construímos então, por indução em $i$, usando o Lema 7.7, conjuntos finitos $G_{i, j}$ para cada $j<K$ e $s_{N}^{i} \in \mathbb{S}$ tais que $G_{0, j}=G_{\sigma_{j}}^{*}, G_{i+1, j} \subseteq G_{i, j}$, $s_{N}^{0}=t_{N}^{\prime}, s_{N}^{i+1} \leq_{F_{N}, N} s_{N}^{i} \mathrm{e}$

$$
s_{N}^{i+1} \mid \sigma_{i} \Vdash \bigcap\left\{\dot{X}_{k, \sigma_{j}}: k \in G_{i+1, j}, j<2^{N}\right\} \text { é infinito. }
$$

Fixemos $0<i<K$. Suponha então que temos $G_{i, j}$ para $j<K \operatorname{com}\left|G_{i, j}\right|$ suficientemente grande e $s_{N}^{i}$ satisfazendo o queremos. Vamos construir $G_{i+1, j}$ e $s_{N}^{i+1}$. Temos que

$$
s_{N}^{i} \mid \sigma_{i} \Vdash \dot{X}_{N} \subseteq \bigcup_{k \in F} \dot{X}_{k, \sigma_{j}},
$$

para todo $F \subseteq G_{\sigma_{j}}^{*} \operatorname{com}|F| \geq m$, pois se existissem $j<K, l \in \mathbb{N}$ e $\left(s_{N}^{i} \mid \sigma_{i}\right)^{\prime} \leq s_{N}^{i} \mid \sigma_{i}$ tais que

$$
\left(s_{N}^{i} \mid \sigma_{i}\right)^{\prime} \Vdash l \in \dot{X}_{N} \backslash \bigcup_{k \in F} \dot{X}_{k, \sigma_{j}}
$$

teríamos que

$$
\left(s_{N}^{i} \mid \sigma_{i}\right)^{\prime} \Vdash\left|\dot{\mu}_{l}\right|\left(\bigcup_{k \in F} \check{A}_{k}\left(\sigma_{j}\right)\right) \geq \sum_{k \in F}\left|\dot{\mu}_{l}\right|\left(\check{A}_{k}\left(\sigma_{j}\right)\right) \geq m \eta \geq M
$$

contradizendo nossa hipótese. Como $\left|G_{i, j}\right|$ é suficientemente grande, pelo Lema 7.7, existe $s_{N}^{i+1} \leq_{N} s_{N}^{i}$ e existem $G_{i+1, j} \subseteq G_{i, j}$ com $\left|G_{i+1, j}\right|$ suficientemente grande, tais que

$$
s_{N}^{i+1} \mid \sigma_{i} \Vdash \bigcap\left\{\dot{X}_{k, \sigma_{j}}: j<K, k \in G_{i+1, j}\right\} \text { é infinito. }
$$

Assim, supondo $C$ suficientemente grande, temos que $\left|G_{K-1, i}\right| \geq 1$ para todo $0 \leq i<$ $K$.

Consideremos então, para cada $0 \leq i<K, k_{i} \in G_{K-1, i}$. Definimos então, $s_{N+1}=s_{N}^{K}$, $a_{N+1}=\bigcup_{i<K} A_{k_{i}}\left(\sigma_{i}\right), E_{N+1}=\left\{k_{i}: i<K\right\}$, para cada $k \in \mathbb{N}, \dot{A}_{k}^{\prime}$ nomes para $\dot{A}_{k} \backslash a_{N+1} \mathrm{e}$ 
$\dot{X}_{N+1}$ um nome tal que

$$
s_{N+1} \mid \sigma_{K-1} \Vdash \dot{X}_{N+1}=\bigcap\left\{\dot{X}_{k, \sigma_{j}}: k \in G_{K, j}, j<K\right\} \backslash \check{E}_{N+1} .
$$

Vejamos que temos tudo o que queremos: primeiramente, note que $s_{N+1}$ força que $\dot{A}_{k}^{\prime}$ são dois a dois disjuntos, pois força que $\dot{A}_{k}^{\prime} \subseteq \dot{A}_{k}$ e que estes o são, e, portanto, temos (1).

Como $E_{N+1}$ é finito, temos que, para todo $i, j<K, j \neq i$

$$
s_{N+1} \mid \sigma_{i} \Vdash \dot{X}_{N+1} \supseteq \bigcap\left\{\dot{X}_{k, \sigma_{j}}: k \in G_{i+1, j}, j<K\right\} \backslash \check{E}_{N+1} \text { que é infinito, }
$$

Além disso,

$$
\begin{aligned}
s_{N+1}\left|\sigma_{i} \Vdash \forall k \in \dot{X}_{N+1}\right| \dot{\mu}_{k}\left(\dot{A}_{k}^{\prime}\right) \mid & \geq\left|\dot{\mu}_{k}\left(\dot{A}_{k}\right)\right|-\sum_{j<K, j \neq i}\left|\dot{\mu}_{k}\right|\left(\check{A}_{k_{j}}\left(\sigma_{j}\right)\right) \\
& >\varepsilon-K \eta=\varepsilon-\delta .
\end{aligned}
$$

e, portanto, temos (2), usando a Proposição 4.42 e o Lema 4.65. Também pela definição de $E_{N+1}$ e de $\dot{X}_{N+1}$ temos (3).

Temos ainda que vale a propriedade (4), pois

$$
\begin{gathered}
s_{N+1} \Vdash \text { se } k \in \dot{X}_{N+1} \text {, então } \\
\left|\dot{\mu}_{k}\left(\check{a}_{N+1}\right)\right|=\left|\dot{\mu}_{k}\left(\bigcup_{i<K} \check{A}_{k_{i}}\left(\sigma_{i}\right)\right)\right| \leq \sum_{i<K}\left|\dot{\mu}_{k}\right|\left(\check{A}_{k_{i}}\left(\sigma_{i}\right)\right) \leq K \eta \leq \delta,
\end{gathered}
$$

e para verificar (5), note que se $k \in E$, temos que se $j<K$,

$$
s_{N+1}\left|\sigma_{j} \Vdash\right| \dot{\mu}_{k}\left(\check{a}_{N+1}\right)|=| \dot{\mu}_{k}\left(\bigcup_{i<K} \check{A}_{k_{i}}\left(\sigma_{i}\right)\right)\left|\leq \sum_{i<K}\right| \dot{\mu}_{k}\left(\check{A}_{k_{i}}\left(\sigma_{i}\right)\right) \mid \leq K \eta \leq \delta,
$$

e daí, pela Proposição 4.42 e o Lema 4.65 temos (5).

Por fim, para cada $i<K$,

$$
\begin{aligned}
s_{N+1} \mid \sigma_{i} \Vdash & \left|\dot{\mu}_{k_{i}}\left(a_{N+1}\right)\right| \geq\left|\dot{\mu}_{k_{i}}\left(\check{A}_{k_{i}}\left(\sigma_{i}\right)\right)\right|-\sum_{j<K, j \neq i}\left|\dot{\mu}_{k_{i}}\right|\left(\check{A}_{k_{j}}\left(\sigma_{j}\right)\right) \\
& >\varepsilon-K \eta=\varepsilon-\delta,
\end{aligned}
$$

e daí, segue (6), novamente pela Proposição 4.42 e o Lema 4.65. 
Note que os lemas combinatórios apresentados nesta seção foram utilizados para provar a proposição anterior. A partir dela, a demonstração do resultado principal se parece com a demonstração do fato que se $A$ é uma álgebra de Boole com a PCS, então $C(S(A))$ tem a propriedade de Grothendieck (Teorema 3.47). Fazemos apenas uma troca de uma família de conjuntos por outra e seguimos o mesmo caminho. Vejamos, finalmente, a demonstração do resultado principal.

Teorema 7.12. Seja $\kappa>\omega_{1}$ um cardinal regular. Seja $G$ um filtro $\mathbb{S}^{\kappa}$-genérico sobre um modelo $M$. Então, temos que em $M[G]$, se $K$ é o espaço de Stone da álgebra $\wp(N) \cap M$, então $C(K)$ é um espaço de Grothendieck de densidade $\omega_{1} \leq 2^{\omega}=\kappa$.

Demonstração. Trabalhamos em $M[G]$ :

Sabemos, pela Proposição 4.70, que $|\wp(\mathbb{N}) \cap M|=\omega_{1}<\kappa=2^{\omega}$. Daí, pela Proposição 1.55, $p(K)=\omega_{1}<2^{\omega}$. Logo, pela Proposição 3.4, $C(K)$ tem densidade $\omega_{1}<\kappa$.

Pelo Teorema de Representação de Riesz (3.13), temos que $C(K)^{*}$ é isométrico ao espaço de medidas de Radon sobre $K$. Suponha, por absurdo, que $\left(\mu_{n}\right)_{n \in \mathbb{N}}$ é uma seqüência de medidas de Radon que não converge fracamente mas que é fraca* convergente a uma medida de Radon $\mu$. Identificamos a álgebra $C l o p(K)$ com a a álgebra $\wp(\mathbb{N})$.

Daí, pelo Lema 3.19 , temos que existem $\varepsilon>0,\left(A_{n}\right)_{n \in \mathbb{N}} \subseteq \wp(\mathbb{N}) \cap M$ e $\left(k_{n}\right)_{n \in \mathbb{N}} \subseteq \mathbb{N}$ crescente tais que

$$
\forall n \in \mathbb{N} \quad\left|\mu_{k_{n}}\left(A_{n}\right)\right| \geq \varepsilon
$$

Notemos que temos a existência de tais $A_{n}$ 's em $M[G]$. Assim, a existência deles é forçada por um elemento de $G$. Temos então que algum elemento de $G$ força algo sobre nomes para elementos da álgebra $\wp(\mathbb{N}) \cap M$. Precisamos, a partir destes nomes, que são candidatos a elementos de $\wp(\mathbb{N}) \cap M$ satisfazendo algumas propriedades, obter, em $M$, elementos de $\wp(\mathbb{N})$ que possuem tais propriedades em $M[G]$. Vejamos:

Trabalhamos em $M$ :

Tomemos $\dot{\mu}_{n}$ nomes para os elementos da seqüência $\left(\mu_{n}\right)_{n \in \mathbb{N}}$ e consideremos as restrições de $\dot{\mu}_{k}$ 's à álgebra $\wp(\mathbb{N})$. Sejam então $s \in \mathbb{S}^{\kappa}$ e $\dot{A}_{n}$ nomes para elementos de $\wp(\mathbb{N}) \cap M$ tais que

$$
s \Vdash \forall n \in \mathbb{N} \quad\left|\dot{\mu}_{n}\left(\dot{A}_{n}\right)\right| \geq \varepsilon .
$$


Temos então nossos candidatos. A proposição anterior garantirá que podemos obter, de fato, aqueles elementos de $\wp(\mathbb{N})$ em $M$, como queremos.

Vamos construir seqüências $\left(s_{N}\right)_{N \in \mathbb{N}}, \operatorname{com} s_{N+1} \leq_{F_{N}, N} s_{N}$, onde $F_{N}=\left\{\alpha_{i}^{k}: i, k<N\right\}$, e $\operatorname{supp}\left(s_{N}\right)=\left\{\alpha_{k}^{N}: k \in \mathbb{N}\right\},\left(a_{N}\right)_{N \in \mathbb{N}}$ dois a dois disjuntos, e $\left(E_{N}\right)_{N \in \mathbb{N}}$ finitos dois a dois disjuntos e $\left(\dot{X}_{N}\right)_{N \in \mathbb{N}}$ uma seqüência de nomes, tais que

$$
s_{N+1} \Vdash \exists k \in \check{E}_{N+1}\left\{\begin{array}{l}
\left|\dot{\mu}_{k}\left(\check{a}_{N+1}\right)\right| \geq \frac{3 \varepsilon}{4} \\
\check{E}_{N+1} \subseteq \dot{X}_{N} \backslash \dot{X}_{N+1}, \\
\text { se } i<N \text { e } k \in \check{E}_{i}, \text { então }\left|\dot{\mu}_{k}\left(\check{a}_{N+1}\right)\right| \leq \frac{\varepsilon}{4}
\end{array}\right.
$$

Suponhamos então que temos construídos $s_{0}, \ldots, s_{N}, a_{1}, \ldots, a_{N}, E_{1}, \ldots, E_{N}, \dot{X}_{0}, \ldots$, $\dot{X}_{N}$ e $\left(\dot{A}_{n}^{N}\right)_{n \in \mathbb{N}}$ como queremos. Aplicamos a Proposição 7.11, com $\varepsilon \operatorname{como} \varepsilon-\sum_{i=1}^{N-1} \frac{\varepsilon}{2^{i+2}}$, $E=\bigcup_{i<N+1} E_{i}, \dot{A}_{k}=\dot{A}_{k}^{N}$ e $\delta$ como $\frac{\varepsilon}{2^{N+2}}$. Temos que existe $s_{N+1} \leq_{F_{N}, N} s_{N}, a_{N+1} \subseteq \mathbb{N}$, $E_{N+1} \subseteq \mathbb{N}$ com $\left|E_{N+1}\right|=2^{N\left|F_{N}\right|}$, uma seqüência $\left(\dot{A}_{n}^{N+1}\right)_{n \in \mathbb{N}}$ de nomes para subconjuntos de $\mathbb{N}$ e $\dot{X}_{N+1}$ um nome tais que

$$
s_{N+1} \Vdash\left\{\begin{array}{l}
\forall k, k^{\prime} \in \mathbb{N} \quad k \neq k^{\prime} \quad \dot{A}_{k}^{N+1} \cap \dot{A}_{k^{\prime}}^{N+1}=\emptyset \\
\left\{k \in \dot{X}_{N}:\left|\dot{\mu}_{k}\left(\dot{A}_{k}^{N+1}\right)\right| \geq \varepsilon-\sum_{i=1}^{N} \frac{\varepsilon}{2^{i+2}}\right\} \supseteq \dot{X}_{N+1} \text { é infinito, } \\
\check{E}_{N+1} \subseteq \dot{X}_{N} \backslash \dot{X}_{N+1} \\
\forall k \in \dot{X}_{N+1} \quad\left|\dot{\mu}_{k}\left(\check{a}_{N+1}\right)\right| \leq \frac{\varepsilon}{2^{N+2}} \\
\forall k \in \bigcup\left\{\check{E}_{i}: i \leq N\right\} \quad\left|\dot{\mu}_{k}\left(\check{a}_{N+1}\right)\right| \leq \frac{\varepsilon}{2^{N+2}} \\
\exists k \in \check{E}_{N+1} \quad\left|\dot{\mu}_{k}\left(\check{a}_{N+1}\right)\right|>\varepsilon-\sum_{i=1}^{N} \frac{\varepsilon}{2^{i+2}}
\end{array}\right.
$$

Obtemos, assim, a seqüência desejada.

Uma vez que obtemos os elementos de $\wp(\mathbb{N})$ em $M$ com as propriedades que queremos, faremos a demonstração seguindo o mesmo caminho feito na demonstração do Teorema 3.47. Mas ainda precisaremos ir de $M$ para $M[G]$ e vice-versa algumas vezes. Vejamos porque:

Tomemos então, pelo Lema $4.63, s^{*} \in \mathbb{S}^{\kappa}$ tal que $s^{*} \leq s_{N}$ para todo $N \in \mathbb{N}$. Consideremos então $\left(K_{\alpha}\right)_{\alpha<\omega_{1}} \subseteq \wp(\mathbb{N})$ uma família quase disjunta. Mas, para todo $\alpha<\omega_{1}$ tem-se que

$$
s^{*} \Vdash \sum_{n \in \check{K}_{\alpha}} \check{a}_{n} \in \wp(\mathbb{N}) \cap M,
$$


e, pela Proposição 4.69,

$s^{*} \Vdash\left(\check{K}_{\alpha}\right)_{\alpha<\check{\omega}_{1}}$ é uma família quase disjunta de subconjuntos de $\mathbb{N}$ e $\check{\omega}_{1}=\omega_{1}$.

Temos aqui as hipótese do Lema 3.35 satisfeitas em $M[G]$. Assim, podemos aplicá-lo lá obtendo a existência de um ordinal $\alpha<\check{\omega}_{1}$ como na tese de tal lema, para depois voltar para $M$ e decidir que será este ordinal.

Trabalhamos, novamente em $M[G]$ e temos que, pelo Lema 3.35 , existe $\alpha<\check{\omega}_{1}$ tal que

$$
\forall k \in \mathbb{N} \quad \dot{\mu}_{k}\left(\sum_{n \in \check{K}_{\alpha}} \check{a}_{n}\right)=\sum_{n \in \check{K}_{\alpha}} \dot{\mu}_{k}\left(\check{a}_{n}\right) .
$$

Voltando para $M$, podemos obter, $s^{* *} \leq s^{*}, s^{* *} \in G$ e $\alpha \in \omega_{1}$ tais que

$$
s^{* *} \Vdash \forall k \in \mathbb{N} \quad \dot{\mu}_{k}\left(\sum_{n \in \check{K}_{\alpha}} \check{a}_{n}\right)=\sum_{n \in \check{K}_{\alpha}} \dot{\mu}_{k}\left(\check{a}_{n}\right) .
$$

Estamos prontos agora para terminar o mesmo caminho seguido na demonstração do Teorema 3.47 , sem ir mais para $M[G]$.

Tomemos

$$
a=\sum\left\{a_{n}: n \in K_{\alpha}\right\}
$$

e vejamos que em $M[G]$, existem infinitos $k \in \mathbb{N}$ e infinitos $l \in \mathbb{N}$ tais que sendo $\delta=\varepsilon / 4$, temos

$$
\left|\mu_{k}(a)\right| \geq 2 \delta \text { e }\left|\mu_{l}(a)\right| \leq \delta
$$

e, portanto, pelo Lema 3.18 , temos que $\left(\mu_{n}\right)_{n \in \mathbb{N}}$ não converge na topologia fraca*, contradizendo nossa hipótese.

Se $i \in K_{\alpha}$, então temos,

$$
s^{* *} \Vdash \begin{cases}\exists k \in \check{E}_{i} & \left|\dot{\mu}_{k}\left(\check{a}_{i}\right)\right| \geq \frac{3 \varepsilon}{4}, \text { por }(6) \\ \forall k \in \check{E}_{i} \quad \forall n<i \quad k \in \dot{X}_{n}, \text { por }(4) \text { e, portanto, }\left|\dot{\mu}_{k}\left(\check{a}_{n}\right)\right| \leq \frac{\varepsilon}{2^{n+2}}, \\ \forall k \in \check{E}_{i} \quad \forall n>i \quad\left|\dot{\mu}_{k}\left(\check{a}_{n}\right)\right| \leq \frac{\varepsilon}{2^{n+2}}, \text { por }(5),\end{cases}
$$

e daí, $s^{* *} \Vdash \exists k \in \check{E}_{i}$ tal que

$$
\begin{aligned}
\left|\dot{\mu}_{k}(\check{a})\right| & =\left|\sum_{n \in \check{K}_{\alpha}} \dot{\mu}_{k}\left(\check{a}_{n}\right)\right| \geq\left|\dot{\mu}_{k}\left(\check{a}_{i}\right)\right|-\sum_{n \in \check{K}_{\alpha} \backslash\{i\}}\left|\dot{\mu}_{k}\left(\check{a}_{n}\right)\right| \\
& \geq \frac{3 \varepsilon}{4}-\sum_{n \in \check{K}_{\alpha} \backslash\{i\}} \frac{\varepsilon}{2^{n+2}} \geq \frac{3 \varepsilon}{4}-\frac{\varepsilon}{4}=2 \delta .
\end{aligned}
$$


Por outro lado, se $i \notin K_{\alpha}$, então,

$$
s^{* *} \Vdash \forall k \in \check{E}_{i} \begin{cases}\forall n<i & k \in \dot{X}_{n} \text { e, portanto, }\left|\dot{\mu}_{k}\left(\check{a}_{n}\right)\right| \leq \frac{\varepsilon}{2^{n+2}} \\ \forall n>i & \left|\dot{\mu}_{k}\left(\check{a}_{n}\right)\right| \leq \frac{\varepsilon}{2^{n+2}}\end{cases}
$$

e daí,

$$
s^{* *} \Vdash \forall k \in \check{E}_{i} \quad\left|\dot{\mu}_{k}(\check{a})\right|=\left|\sum_{n \in \check{K}_{\alpha}} \dot{\mu}_{k}\left(\check{a}_{n}\right)\right| \leq\left|\sum_{n \in \check{K}_{\alpha}} \frac{\varepsilon}{2^{n+2}}\right| \leq \frac{\varepsilon}{4}=\delta .
$$

Portanto, segue o resultado. 


\section{Referências Bibliográficas}

[Ai] AIZPURU, A. The Nikodým property and local properties of Boolean algebras. Colloq. Math., v.71, n.1, p.79-85, 1996.

[Ba] BAUMGARTNER, J. E. Sacks forcing and the total failure of Martin's axiom. Topology Appl., v.19, n.3, p.211-225, 1985.

[Bre] BRECH, C. A densidade de espaços de Grothendieck da forma $C(K)$. In: SEMiNÁRIO BRASILEIRO DE ANÁliSE, 58, 2003, Campinas. Trabalhos Apresentados ..., Campinas: 2003, p.539-546.

[Ci] CIESIELSKI, K. Set theory for the working mathematician. (London Mathematical Society student texts, 39) Cambridge University Press, Cambridge, 1997. xii+236p.

[Co1] COHEN, P. J. The independence of the continuum hypothesis. Proc. of Nat. Acad. Sci. U.S.A. v.50, p.1143-1148, 1963.

[Co2] COHEN, P. J. The independence of the continuum hypothesis II. Proc. Nat. Acad. Sci. U.S.A. v.51, p.105-110, 1964.

[DW] DALES, H. G.; WOODIN, W. H. An introduction to independence for analysts. (London Mathematical Society Lecture Note Series, 115) Cambridge University Press, Cambridge, 1987. xiv+241p.

[Di] DIESTEL, J. Sequences and series in Banach spaces. (Graduate Texts in Mathematics, 92), Springer-Verlag, New York, 1984. xii+261p.

[DU] DIESTEL, J. UHL, J. Vector measures, (Mathematical Surveys, 15) American Mathematical Society, Providence, 1977. xiii+322p. 
[Do1] DOW, A. Saturated Boolean algebras and their Stone spaces. Topology Appl. v.21, n.2, p.193-207, 1985.

[Do2] DOW, A. An introduction to applications of elementary submodels to topology. Topology Proc. v.13, n.1, p.17-72, 1988.

[DR] DREWNOWSKI, L.; ROBERTS, J. On the primariness of the Banach space $l_{\infty} / c_{0}$. Proc. Amer. Math. Soc. v.112, n.4, p.949-957, 1991.

[Ek1] EKLOF, P. C. Whitehead's problem is undecidable. Amer. Math. Monthly v.83, n.10, p.775-788, 1976.

[Ek2] EKLOF, P. C. Set-theoretic methods in homological algebra and abelian groups. (Séminaire de Mathématiques Supérieures, 69) 1980, Presses de 1'Université de Montréal, Montreal, 1980. 117p.

[End] ENDERTON, H. B. A mathematical introduction to logic. Academic Press, New York, 1972. xiii+295p.

[Eng] ENGELKING, R. General topology. 2nd. ed. (Sigma Series in Pure Mathematics, 6), Heldermann Verlag, Berlin, 1989. viii+529p.

[Go] GÖDEL, K. What is Cantor's continuum problem? Amer. Math. Monthly v.54, p.515-525, 1947.

[Gro] GROTHENDIECK, A. Sur les applications linéaires faiblement compactes d'espaces du type $C(K)$. Canadian J. Math. v.5, p.129-173, 1953.

[Ha] HAYDON, R. A non-reflexive Grothendieck space that does not contain $l_{\infty}$. Israel J. Math. v.40, n.1, p.65-73, 1981.

[HLO] HAYDON, R.; LEVY, M.; ODELL, E. On sequences without weak* convergent convex block subsequences. Proc. Amer. Math. Soc. v.100, n.1, p.94-98, 1987.

[JK] JUST, W.; KOSZMIDER, P. Remarks on cofinalities and homomorphism types of Boolean algebras. Algebra Universalis v.28, n.1, p.138-149, 1991.

[Kopp] KOPPELBERG, S. Handbook of Boolean algebras. v.1, North-Holland Publishing Co., Amsterdam, 1989. xx+312lp. 
[Kosz] KOSZMIDER, P. Banach spaces with few operators. Math. Ann. To appear.

[Kre] KREYSZIG, E. Introductory functional analysis with applications. (Wiley Classics Library) John Wiley and Sons, New York, 1989. xvi+688p.

[Ku] KUNEN, K. Set theory. An introduction to independence proofs. (Studies in Logic and the Foundations of Mathematics, 102) North-Holland Publishing Co., Amsterdam, 1980. xvi+313p.

[La] LAVER, R. On the consistency of Borel's conjecture. Acta Math. v.137, p.151-169, 1976.

[LW] LEONARD, I. E.; WHITFIELD, J. H. M. A classical Banach space: $l_{\infty} / c_{0}$. Rocky Mountain J. Math. v.13, n.3, p.531-539, 1983.

[Ma] MASCIONI, V. Topics in the theory of complemented subspaces in Banach spaces. Exposition. Math. v.7, n.1, p.3-47, 1989.

[Me] MEGGINSON, R.E. An introduction to Banach space theory. (Graduate Texts in Mathematics, 183) Springer-Verlag, New York, 1998.

[vM] van MILL, J. An introduction to $\beta \omega$. In: KUNEN, K.; VAUGHAN, J. E. (Eds). Handbook of set-theoretic topology. North-Holland, Amsterdam, 1984. p.503567.

[Ne] NEGREPONTIS, S. The Stone space of the saturated Boolean algebras. Trans. Amer. Math. Soc. v.141, 1969, p.515-527.

[Roi] ROITMAN, J. Introduction to modern set theory. (Pure and Applied Mathematics) John Wiley and Sons, New York, 1990. xiv+156p.

[Ros1] ROSENTHAL, H. P. On relatively disjoint families of measures, with some applications to Banach space theory. Studia Math. v.37, p.13-36, 1970.

[Ros2] ROSENTHAL, H. P. The Banach spaces $C(K)$. In: JOHNSON, W. B.; LINDENSTRAUSS, J. (Eds). Handbook of the geometry of Banach spaces. v.2, North-Holland Publishing Co., Amsterdam, 2003. p.1547-1602. 
[Sa] SACKS, G. E. Forcing with perfect closed sets. Axiomatic Set Theory(Proc. Symp. Pure Math., v.13, Part I, Univ. California, Los Angeles, Calif., 1967) p.331355, 1971.

[Scha] SCHACHERMAYER, W. On some classical measure-theoretic theorems for non-sigma-complete Boolean algebras. (Dissertationes Math., 214.) 1982. $33 \mathrm{p}$.

[Se] SEMADENI, Z. Banach spaces of continuous functions. v.1, (Monografie Matematyczne Tom 55) PWN Polish Scientific Publishers, Warsaw, 1971. 584p.

[Ta] TALAGRAND, M. Un nouveau $C(K)$ qui possède la propriété de Grothendieck. Israel J. Math. v.37, n.1-2, p.181-191, 1980.

[We] WEISS, W. Versions of Martin's axiom. In: KUNEN, K.; VAUGHAN, J. E. (Eds). Handbook of set-theoretic topology. North-Holland, Amsterdam, 1984. p.827886.

[Zip] ZIPPIN, M. Extension of bounded linear operators. In: JOHNSON, W. B.; LINDENSTRAUSS, J. (Eds). Handbook of the geometry of Banach spaces. v.2, North-Holland Publishing Co., Amsterdam, 2003. p.1703-1741.

[Ziz] ZIZLER, v. Nonseparable Banach spaces. In: JOHNSON, W. B.; LINDENSTRAUSS, J. (Eds). Handbook of the geometry of Banach spaces. v.2, NorthHolland Publishing Co., Amsterdam, 2003. p.1743-1816. 


\section{Índice Remissivo}

$$
\begin{aligned}
& \left(X, \sigma\left(X, X^{*}\right)\right), 40 \\
& \left(X^{*}, \sigma\left(X^{*}, X\right)\right), 42 \\
& (\omega, 1) \text {-abertura, } 143 \\
& (\omega, \omega) \text {-abertura, } 143 \\
& C \operatorname{lop}(K), 26
\end{aligned}
$$$$
\text { Con }(T), 92
$$$$
M[G], 98
$$$$
\operatorname{Ult}(A), 12
$$$$
X \equiv Y, 39
$$$$
X \sim Y, 38
$$$$
Y \oplus Z, 45,47
$$$$
\Gamma, 102
$$$$
\Phi^{M}, 92
$$$$
\Vdash, 104
$$$$
\Vdash^{*}, 106
$$$$
\perp, 96
$$$$
\kappa \text {-c.c., } 116
$$$$
\kappa \text {-fechado, } 116
$$$$
\leq_{n}, 120
$$$$
\leq_{F, n}, 122
$$$$
\check{x}, 101
$$$$
\omega^{*}, 140
$$

$\sigma$-fechado, 116

₹, 93

$\vdash, 92$

$a \Delta b, 14$

$a^{*}, 23$ bif $(p, s), 120$

$d(K), 20$

$l(n, s), 120$

$l_{\infty}(X), 48$

n-ésimo nível de bifurcação, 120

$p(K), 22$

$\operatorname{val}(\tau, G), 98$

álgebra de Boole, 7

álgebra de Boole

$\sigma$-completa, 17

atômica, 34

completa, 17

quociente, 14

superatômica, 34

árvore perfeita, 120

átomo, 34

ínfimo, 17

forcing, 96

forcing

de Cohen, 117

de Sacks, 120

teorema de, 95

aberto, 124

absoluta, 100

Alaoglu

teorema de, 43

anticadeia, 17 


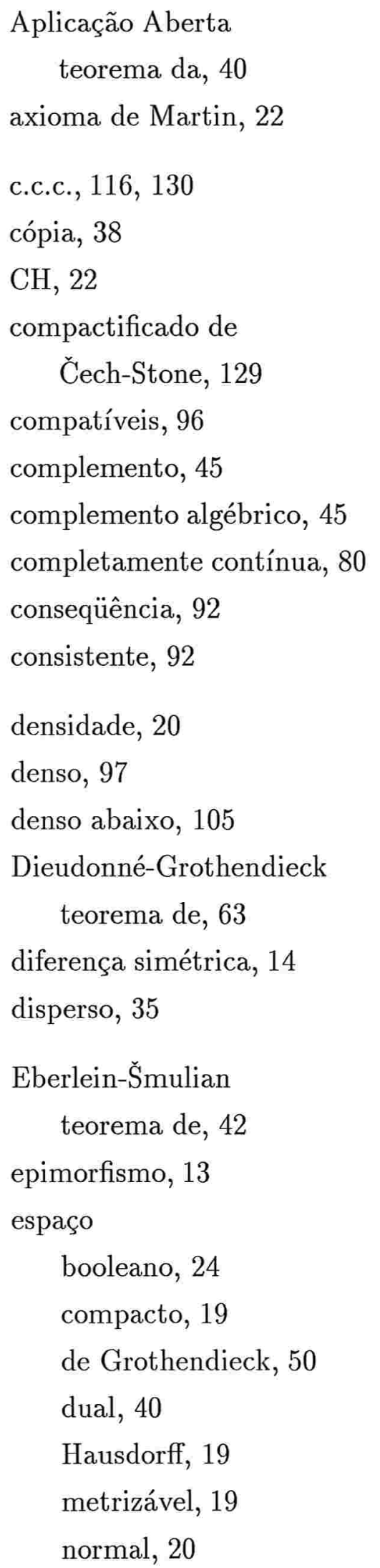

quociente, 49

separável, 20

zero-dimensional, 19

espaço de Stone, 24

extensão, 96

extremamente desconexo, 34

família independente, 32

fecho para baixo, 120

filtro, 11, 97

filtro maximal, 11

força, 104

forma normal, 9

fracamente

compacta, 44

compacto, 42

convergente, 40

nula, 41

seqüencialmente compacto, 42

fracamente*

compacto, 43

convergente, 42

função simples, 57

fusão

lema de, 120, 123

Gödel

teorema da Completude de, 93

Gantmacher

teorema de, 44

genérico, 97

Gráfico Fechado

teorema do, 40

Hahn-Banach 
teorema de, 39

hipótese do contínuo, 22

homomorfismo, 13

homomorfismo

teorema do, 14

ideal, 14

imagem homomorfa, 13

incompatíveis, 96

inconsistente, 92

independente, 96

injetivo, 72

integral de $f$ com respeito a $\mu, 58,59$

isometria, 38

isomorfas, 13

isomorfismo, 13, 38

isomorfos, 38

Lebesgue

teorema da Convergência Dominada

$$
\text { de, } 61
$$

lema

de fusão, 120,123

de Rosenthal, 75

Limitação Uniforme

teorema de, 39

máximo, 96

MA, 22

medida, 58

medida

boreliana, 59

de Radon, 59

finitamente aditiva, 58

limitada, 58 regular, 59

modelo, 93

modelo transitivo, 94

monomorfismo, 13

nível de bifurcação, 120

Nachbin-Goodman

teorema de, 72

nome, 97

operador adjunto, 43

PCS, 86

Pełczyński

teorema de Decomposição de, 48

peso, 22

preserva cardinais, 116

primário, 156

produto elementar, 9

projeção, 45

propriedade

de completude subseqüencial, 86

de Dunford-Pettis, 78

de Grothendieck, 50

de intersecção finita, 12

relativamente

consistente, 92

fracamente compacto, 42

independente, 96

relativização, 92

retrato, 35

Riesz

teorema de Representação de, 60

Rosenthal 
lema de, 75

satisfaz, 93

Sikorski

teorema da Extensão de, 18

teorema Primeiro critério de extensão de, 15

teorema Segundo critério de extensão de, 15

Sobczyk

teorema de, 68

soma

direta, 45

exterior, 47

Stone

teorema da dualidade de, 30

teorema da Representação de, 26

Stone-Weierstrass

teorema de, 57

subálgebra, 7

subálgebra gerada, 8

subespaço

complementado, 45

gerado, 39

suporte, 122

supremo, 17

teorema

de Decomposição de Pełczyński, 48

da Aplicação Aberta, 40

da Completude de Gödel, 93

da Convergência Dominada de Lebesgue, 61

da dualidade de Stone, 30 da Extensão de Sikorski, 18

da Extensão de Tietze, 21

da Representação de Stone, 26

de forcing, 95

de Alaoglu, 43

de Dieudonné-Grothendieck, 63

de Eberlein-Šmulian, 42

de Gantmacher, 44

de Hahn-Banach, 39

de Limitação Uniforme, 39

de Nachbin-Goodman, 72

de Representação de Riesz, 60

de Sobczyk, 68

de Stone-Weierstrass, 57

de Tychonoff, 21

do Gráfico Fechado, 40

do homomorfismo, 14

Primeiro critério de extensão de Sikorski, 15

Segundo critério de extensão de Sikorski, 15

Tietze

teorema da Extensão de, 21

topologia

de Stone, 24

fraca, 40

fraca*, 42

transitivo, 94

Tychonoff

teorema de, 21

ultrafiltro, 11

variação, 59 
ÍNDICE REMISSIVO

ZFC, 22 